\title{
Structure and photocatalytic activity of flame-synthesized titanium dioxide catalysts
}

Ph.D. Dissertation

Nándor Balázs

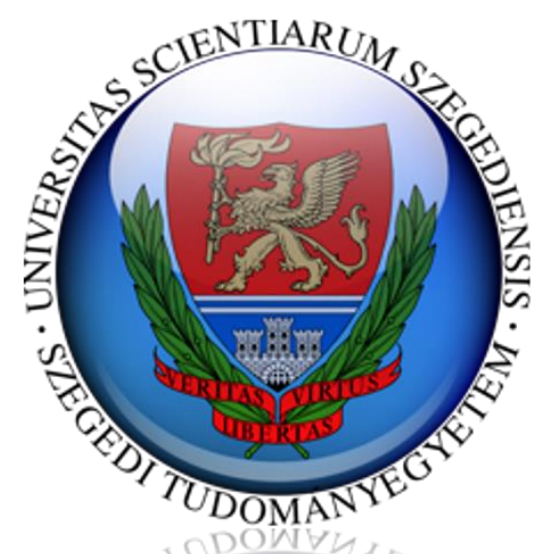

Supervisors:

Dr. Pál Sipos, Dr. Károly Mogyorósi

University of Szeged | Faculty of Science and Informatics

Doctoral School of Environmental Sciences

Department of Inorganic and Analytical Chemistry

Szeged 


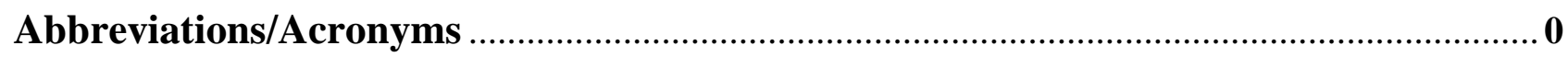

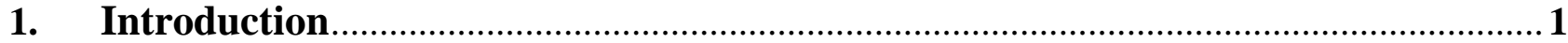

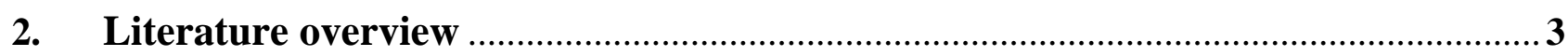

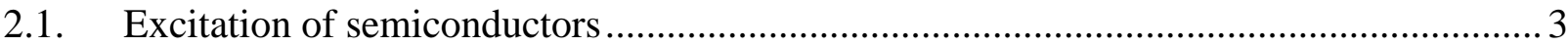

2.2. Charge separation and charge carrier dynamics.............................................................. 3

2.3. Parameters affecting charge recombination ..............................................................

2.4. Interfacial charge transfer and subsequent surface/near-surface reactions in aqueous

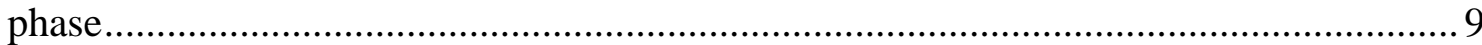

2.5. Decomposition mechanisms of model compounds ...................................................... 13

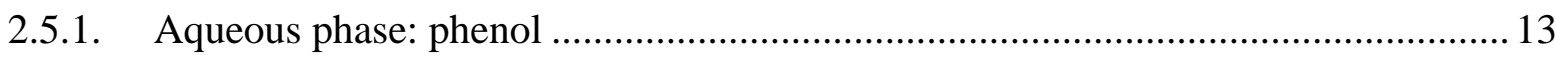

2.5.2. Gas phase: methanol and acetaldehyde................................................................. 16

2.6. Effects of process parameters in aqueous photocatalytic degradation............................. 17

2.7. Comparison of gas and aqueous phase environmental $\mathrm{TiO}_{2}$ applications .......................20

2.8. The role of structural properties of $\mathrm{TiO}_{2}$ in photocatalytic activity ...............................23

2.9. Alternatives of photocatalytically active $\mathrm{TiO}_{2}$ synthesis .............................................24

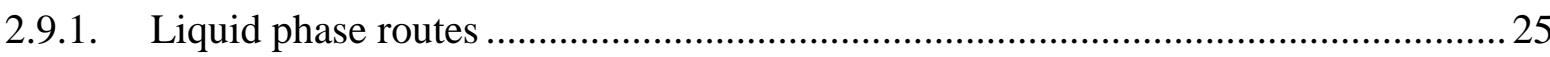

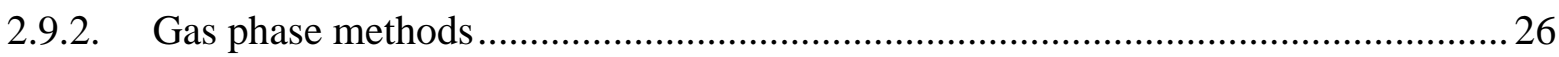

2.10. Gas phase particle formation and particle dynamics in flames.......................................... 31

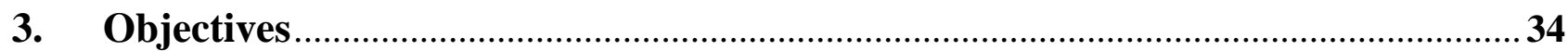

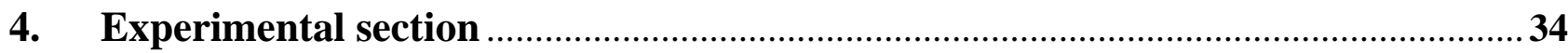

4.1. Preparation of $\mathrm{TiO}_{2}$ samples by vapor-fed aerosol flame synthesis (VAFS) .................. 34

4.2. Determination of photocatalytic activities ..................................................................... 37

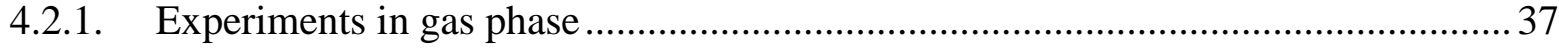

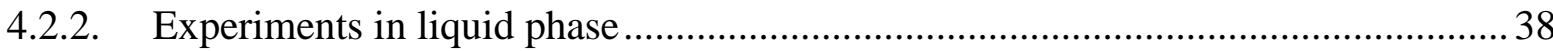

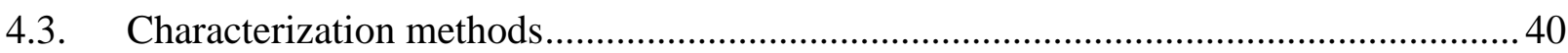

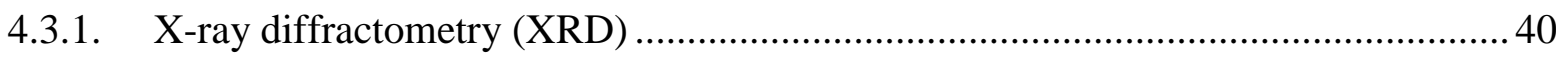

4.3.2. Transmission electron microscopy (TEM) ………………………………….... 41

4.3.3. Scanning electron microscopy (SEM) ………….............................................. 42

4.3.4. High-resolution transmission electron microscopy (HRTEM) ................................ 43

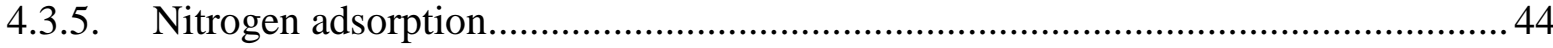

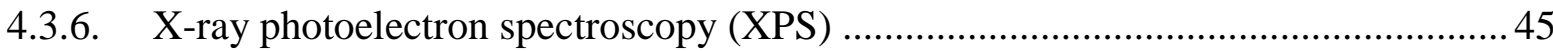

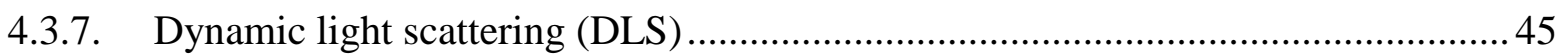


4.3.8. Total organic carbon (TOC) measurements ..................................................... 46

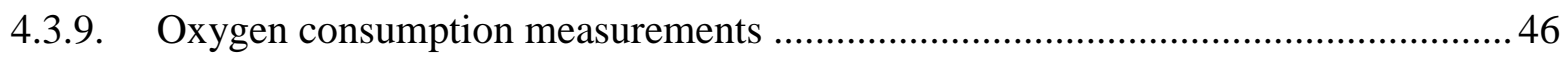

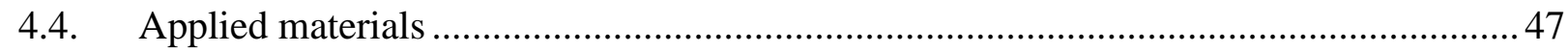

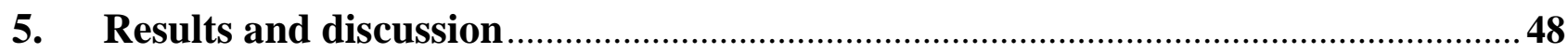

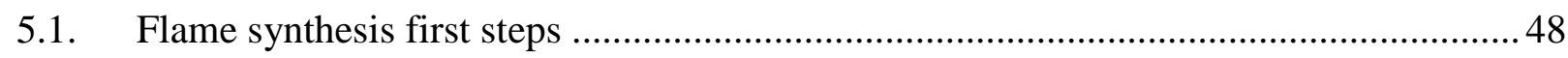

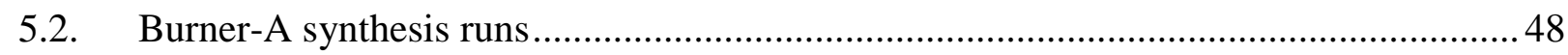

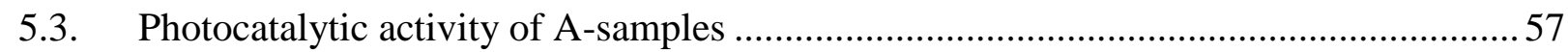

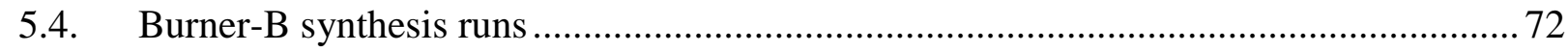

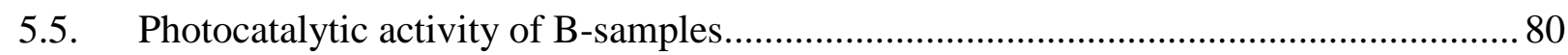

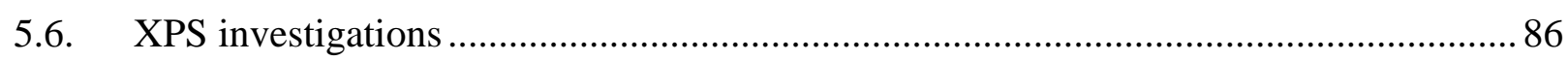

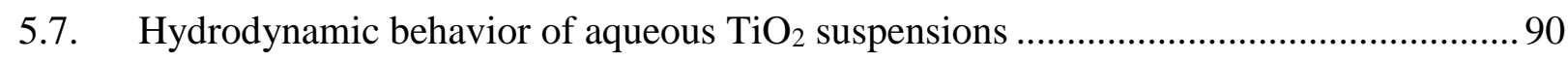

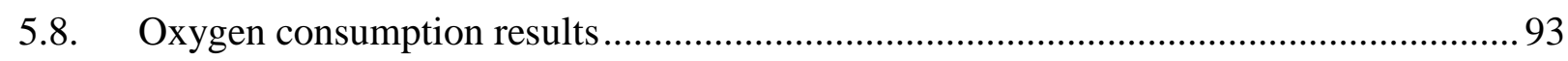

5.9. Mineralization efficiencies and intermediate studies .............................................. 97

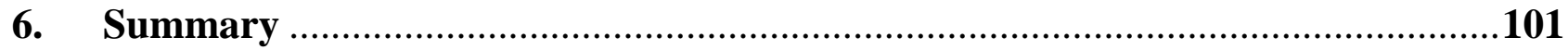

6. Összefoglalás ...............................................................................................107

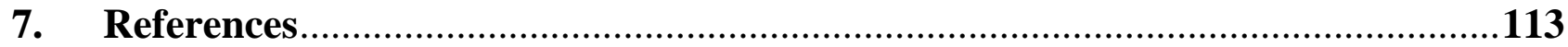

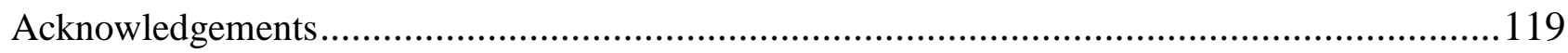




\section{Abbreviations/Acronyms:}

\begin{tabular}{|c|c|}
\hline AFS: & Aerosol Flame Synthesis \\
\hline AOTs: & Advanced Oxidation Technologies \\
\hline BET: & Brunauer-Emmett-Teller \\
\hline CB: & Conduction Band \\
\hline CVD: & Chemical Vapor Deposition \\
\hline DHCHD: & DiHydroxyCycloHexaDienyl \\
\hline DLS: & Dynamic Light Scattering \\
\hline DRIFTS: & Diffuse Reflectance Infrared Fourier Transform Spectroscopy \\
\hline есв": & an Electron, that is energetically localized within the Conduction Band \\
\hline EF: & Fermi-level Energy \\
\hline EG: & Bandgap Energy \\
\hline EPR: & Electron Paramagnetic Resonance \\
\hline FASP: & Flame-Assisted Spray Pyrolysis \\
\hline FTIR: & Fourier Transform InfraRed \\
\hline FWHM: & Full Width at Half Maximum \\
\hline GC: & Gas Chromatography \\
\hline HPLC: & High Performance/Pressure Liquid Chromatography \\
\hline HQ: & HydroQuinone (1,4-Dihydroxybenzene) \\
\hline HRTEM: & High-Resolution Transmission Electron Microscopy \\
\hline HVAC: & Heating, Ventilation, Air Conditioning \\
\hline $\mathbf{h V B}^{+}:$ & a Hole, that is energetically localized within the Valance Band \\
\hline IR: & InfraRed \\
\hline LAFS: & Liquid-fed Aerosol Flame Synthesis \\
\hline OBrH & Bridging Hydroxyl Group \\
\hline ОтН & Terminal Hydroxyl Group \\
\hline PC: & PyroCatechol (1,2-Dihydroxybenzene) \\
\hline pHIEP: & pH of IsoElectric Point \\
\hline PVD: & Physical Vapor Deposition \\
\hline PZC/pHPZc: & Point of Zero Charge \\
\hline RH: & Relative Humidity \\
\hline SCL: & Space-Charge Layer \\
\hline SEM: & Scanning Electron Microscopy \\
\hline SSA: & Specific Surface Area \\
\hline TEM: & Transmission Electron Microscopy \\
\hline TOC: & Total Organic Carbon \\
\hline TTIP: & Titanium TetraIsoPropoxide \\
\hline $\mathbf{U V}:$ & Ultra Violet \\
\hline VAFS: & Vapor-fed Aerosol Flame Synthesis \\
\hline VB: & Valance Band \\
\hline VOCs: & Volatile Organic Compounds \\
\hline XPS: & $\mathbf{X}$-ray Photoelectron Spectroscopy \\
\hline XRD: & $\mathbf{X}$-ray Diffractometry \\
\hline
\end{tabular}




\section{Introduction}

Titanium dioxide $\left(\mathrm{TiO}_{2}\right)$ is one of the semiconductors used in extremely wide range nowadays, its production and application requires a significant industrial capacity. Accordingly, a huge number of fundamental as well as applied research deal with the improvement of its material characteristics through synthesis optimization. The still growing number of publications in this field of science appropriately reflects the great interest and economic potential lying in $\mathrm{TiO}_{2}$-related applications [12].

Each year, quite a few million tons of $\mathrm{TiO}_{2}$ is produced worldwide [3]. Most of this amount (ca. 98\%) is marketed as pigments ("titanium white"), food additives (E171), filling substances (e.g., in plastic industry) and only a few percent represent those fields, where advantageous properties of $\mathrm{TiO}_{2}$ are being exploited in a more specific way. Its chemical stability (inertness) and physical resistance is generally beneficial for each type of application. $\mathrm{TiO}_{2}$ is frequently used as supporting material of different catalysts. It is biocompatible, thus suitable for being utilized as drug coatings and cosmetic additives [1]. Due to its favorable electronic and optical characteristics, $\mathrm{TiO}_{2}$ is increasingly wide-spread base material of solar cells [4] and batteries [5]. Applications in sensorics are also well-known [6-7]. Besides versatility, economic aspects of utilizing $\mathrm{TiO}_{2}$ also play an important role in becoming such a popular material, since industrial-scale production was promoted by low costs of manufacturing and by its high availability [1].

Branches of industry and science dealing with $\mathrm{TiO}_{2}$-assisted photocatalysis exhibited a massive progress over the last few decades. $\mathrm{TiO}_{2}$ is a wide bandgap semiconductor, in which charge separation occurs upon UV-light exposure. These charge carriers (electron-hole pairs) are suitable for initiating redox reactions at the solid-liquid/gas interface. The use of heterogeneous photocatalysis is mainly aimed at the removal of water- and airborne pollutants from our environment. These substances are mostly organics, which can be degraded oxidatively to simple inorganic compounds by photocatalysis. This transformation is called mineralization. The process also shows germicidal activity via destruction of cell walls [8-10].

By now, $\mathrm{TiO}_{2}$-assisted photocatalysis has already proven to be a powerful tool in water and air purification. $\mathrm{TiO}_{2}$ is routinely used as self-cleaning [11-12] and anti-fogging coatings on different surfaces (metal and glass parts of buildings, street tiles, surgery flooring, etc.) [13-15] and it became an essential part of air-cleaning devices [16-17]. Prospectively, its role may get more emphasized in water treatment technologies as well [18-20], complementing or displacing currently employed oxidation techniques, like chlorination and ozonation.

However, heterogeneous photocatalysis is useful not only for decomposing molecules, but for constructing them. Numerous studies on organic chemistry [21-23] proved that sufficiently high 
selectivity and yield can be achieved by using $\mathrm{TiO}_{2}$-based photocatalytic method. This is in particular valid for the synthesis of compounds with oxygen-containing functional groups (alcohols, ketones). Photocatalysis has some advantages over conventional processes, since this way one can get rid of expensive and hazardous solvents and high reaction temperatures. There are a few more fields in photocatalytic synthesis, such as artificial photosynthesis and nitrogen fixation [24], where efforts are also made.

Recently, there are two main threads of photoactive $\mathrm{TiO}_{2}$ improvement: researchers make great effort to enhance the efficiency through decreasing the rate of electron-hole recombination and to expand their excitability towards the visible region of light. Besides these, the demand of further increasing the conversion rate and selectivity of certain photocatalytic reactions sustains an intensive scientific work. For the above purposes, catalyst modifications and additives are often used. The former option means both surface modifications (by attaching organic and inorganic functional groups to it) [25-26] and doping (incorporation of impurities into the crystal lattice) [27-28]. The most important additives are co-adsorbents, sensitizing dyes [29] for ensuring visible light absorption, and co-catalysts forming composite catalyst systems [30-32] with $\mathrm{TiO}_{2}$. 


\section{Literature overview}

\subsection{Excitation of semiconductors}

The invention of $\mathrm{TiO}_{2}$-based photo-induced phenomena [33-36] opened the way for its manifold application as a photocatalyst. The ability of being excited by light irradiation originates from the special electronic properties of semiconductors, which can be explained by using the solid state band structure theory. Atoms, constituting the crystal lattice interact with each other through chemical bonds. Therefore, the individual atomic orbitals are mutually split and merged into bands, which represent quasi-continuous energy levels. Basically, there are two available bands for the electrons to occupy: the valance band (VB) and the conduction band (CB) with lower and with higher energy, respectively. These bands are energetically separated by the so-called bandgap, whose width is material-dependent. It acts as a barrier, so in general (in the case of ground state) conduction cannot be observed as electrons dwell in the valance band. If sufficiently high energy (equal to, or higher than that of the bandgap) is transferred to the system, electrons can get across the bandgap and reach the conduction band. Electron vacancies left behind in the valance band are considered as positively charged "holes" which are also moving when current flows through, this way contributing to the overall conductivity. The migration of holes is actually a consequence of valance electron relocation, since they fill up unoccupied spaces thus leaving a new hole at the original position. [37]

The presence of acceptor or donor type intra-bandgap levels can lower the energy required for the charge separation process (even thermal excitation is possible). These levels can be introduced via doping, i.e., impurities are incorporated into the crystal lattice of a given semiconductor. If so, the material is called extrinsic or doped semiconductor. For the pure/intrinsic semiconductors, the same effect is observed without adding any dopants. Here, certain type of lattice defects can significantly increase the number of charge carriers. For instance, stoichiometric $\mathrm{TiO}_{2}$ has rather low conductivity, however, slight reduction causes a considerable enhancement (by a factor of $10^{9}$ ) in it [38]. The majority carriers in pure $\mathrm{TiO}_{2}$ are electrons, what is referred in its classification: n-type intrinsic semiconductor.

\subsection{Charge separation and charge carrier dynamics}

Photocatalytic processes are initiated by the illumination of $\mathrm{TiO}_{2}$. The energy of the light has to exceed the bandgap width in order to produce separated electron-hole pairs (excitons). These quasiparticles are generated within maximum a few tens of femtoseconds [39] and are responsible for 
catalyzing redox reactions at the solid-medium interface. During charge migration towards the surface, recombination usually occurs to a relatively great extent, resulting in catalytic efficiency loss [40]. This is mainly due to the speed of the process, since recombination proceeds on pico- and nanosecond scale subsequent to excitation [41], being much faster than charge diffusion in bulk or interfacial charge transfer (microseconds to milliseconds) [42-43]. Under optimal conditions the latter two processes are not hindered, so they can compete with recombination. In this case, some charge carriers are able to reach the interface and participate in chemical transformations. It is to be mentioned, that timescale data of charge carrier processes might seem contradictory if one surveys different review articles elaborating the topic [39,41], because those values can apparently be dependent on the measurement technique, catalyst state (single crystal or polycrystalline sample) and quality, light intensity, reaction medium and on chemicals participating in the photocatalytic reaction. For instance, Yoshihara et al. [44] recorded an electron-hole recombination half-life as high as $1 \mu \mathrm{s}$ in nitrogen-saturated water while others measured that to occur in nanoseconds [45].

Charge trapping sites within the crystal lattice play an essential role in recombination and in interfacial charge transfer dynamics. In pure $\mathrm{TiO}_{2}$, two distinct types of both electron and hole trapping modes are known based on the extent of carriers' energy loss when they relax into these states from free state (that is, from CB and VB): deep and shallow. Spectroscopic measurements revealed [46-47] that the time constant of shallow trapping falls within the 100-150 fs range, whereas deep trapping occurs in 100-500 ps, for both carriers.

Electron deep trap states represent mid-bandgap energy levels (Figure 1) while shallow states are located closer to the CB-edge - around 0.4-0.9 eV below that [48-49] -, with higher reduction potential than that of molecular $\mathrm{O}_{2}$ [50]. Electron deep trapping is irreversible and occurs as follows [51]:

$$
\mathrm{e}_{\mathrm{CB}}{ }^{-}+\mathrm{Ti}^{\mathrm{IV}} \rightarrow \mathrm{Ti}^{\mathrm{III}}
$$

Some contradiction exists in the literature with respect to the role of surface OH-groups:

$$
\mathrm{e}_{\mathrm{CB}}{ }^{-}+\mathrm{Ti}^{\mathrm{IV}} \mathrm{OH} \rightarrow\left\{\mathrm{Ti}^{\mathrm{III}} \mathrm{OH}\right\}^{-}
$$

In some cases, this is classified as shallow trapping [1]. On the other hand, Szczepankiewicz and coworkers inferred its - although not exclusively - deep trapping nature [52-53] from O-H stretching modes of titanol groups, studied by DRIFTS (diffuse reflectance FTIR spectroscopy) technique. (The given explanation does not rule out the first option, Reaction 1, being a bulk process.) They also argued, that shallow trap states are less localized, and could equally be ascribed to both hydroxyl 
moieties - bridging $\left(\mathrm{O}_{\mathrm{Br}} \mathrm{H}\right)$ and terminal $\left(\mathrm{O}_{\mathrm{T}} \mathrm{H}\right)$ hydroxyl groups -, as indicated in Reaction 2 . According to their findings, shallow trap state physically corresponds to delocalized electrons being coupled with two-dimensional lattice phonons, to which vibrations of both OH-groups contribute [50]. Shallow trapping is reversible, leading to dynamic charge interchange equilibrium between these states and the CB. This renders surface reduction of adsorbed species possible, namely, such kind of trapping is a prerequisite of $\mathrm{e}_{\mathrm{CB}}{ }^{-}$mediated reactions [52]. Regardless of the manner they are formed, deeply trapped electrons are spatially more localized, promoting annihilation with holes [54]. Irreversible nature of deep trapping is associated with the abated reduction potential of such electrons, being not suitable for reacting further with adsorbed compounds, like oxygen. Shallowly trapped electrons are continuously consumed (if conditions are optimal) by surface redox interactions, therefore competing recombination proceeds to a lower extent.

Surface hydroxyl groups (Reaction 3) and lattice oxygen atoms (Reaction 4) are known to be responsible for hole trapping [55-56].

$$
\begin{gathered}
\mathrm{hVB}^{+}+\mathrm{Ti}^{\mathrm{IV}} \mathrm{OH} \rightarrow\left\{\mathrm{Ti}^{\cdot \mathrm{IV}} \mathrm{OH}^{\cdot}\right\}^{+} \text {(3) } \\
\mathrm{h}_{\mathrm{VB}}{ }^{+}+1 / 2 \mathrm{O}^{2-}{ }_{\text {lattice }} \leftrightarrow 1 / 4 \mathrm{O}_{2}(\mathrm{~g})+\text { oxygen vacancy }
\end{gathered}
$$

Reaction 3 yields surface-bound hydroxyl radicals $(\cdot \mathrm{OH})$. The question that which of the two types of $\mathrm{OH}$-groups could take part in hole trapping cannot be answered straight. The excellent review of Fujishima et al. lists numerous works that provide pros and cons for both possibilities [39]. Oxygen vacancies produced by e.g., the photochemical process (Reaction 4) can rapidly be filled upon dissociative chemisorption of $\mathrm{O}_{2}$ [52] or $\mathrm{H}_{2} \mathrm{O}$ molecules [57], thus healing the defects or forming additional hydroxyl groups, respectively. Latter may be advantageous due to the increased number of trapping sites or, in certain cases, to enhanced surface hydrophilicity. Moreover, OH-groups are considered as potential sources of reactive $\mathrm{OH}$-radicals (Reaction 3). From energetics point of view, researchers assign states found 1.3-1.5 eV above the VB-edge to deeply trapped holes [58-59]. However, there is no clear information on shallow state levels, probably due to their short lifetime [39]. An important experimental deduction is that almost all photogenerated holes are trapped in deep or shallow states, a large fraction of photogenerated electrons are in a nearly free, untrapped state [60].

As described before, most theories involve trapping as a process required for the annihilation of opposing charges [53] as well as for interfacial charge transfer. Faster trapping and shorter residence time in such states are usually believed to provide higher catalytic efficiency, since there is less probability of recombination in lack of charge accumulation [61]. 
Naturally, there are many factors - including structural characteristics of the catalysts and the circumstances applied during catalytic tests - determining the overall photocatalytic performance and also having influence on charge separation. In the next chapter we will give a brief overview on parameters affecting the charge carrier recombination rate in $\mathrm{TiO}_{2}$. Prior to this, a few words have to be told about some internal electronic phenomena provoked by the interaction with the surrounding media. When a semiconductor is exposed to an aqueous electrolyte, or if it is in contact with a semiconductor/conductor, the so-called space-charge layer (SCL) is formed within the outermost region of the solid [62]. This layer extends directly from the interface towards the bulk and represents a potential change along itself; in other words, an electric field is built up at the junction. The phenomenon can suitably be demonstrated by the band-bending effect, meaning a gradual increase or decrease (depending on the nature of interacting systems) of bulk energy levels (VB, CB) in heading the surface (Figure 1). The reason behind is the interfacial charge transfer through which equilibrium state is established. By that, the Fermi-level $\left(\mathrm{E}_{\mathrm{F}}\right)$ of the semiconductor and the redox potential of the electrolyte - or the Fermi-level of an adjoining solid material - eventually become equal. Due to its lower conductivity, potential drop inevitably occurs inside the solid, leading to the band-bending described. The as-generated electrostatic field forces holes and electrons to remain spatially separated, therefore recombination becomes somewhat hindered.

The example of semiconductor-electrolyte junction taken for the above explanation was chosen arbitrarily, similar mechanisms have to be considered at solid-solid interfaces. In Figure 1, we show a schematic drawing of semiconductor energy levels participating in photophysical processes, when being in contact with an electrolyte. Relative energy positions of the individual states indicated therein are not accurate, they are only intended to reflect approximate conditions that were deduced from papers referred above. 


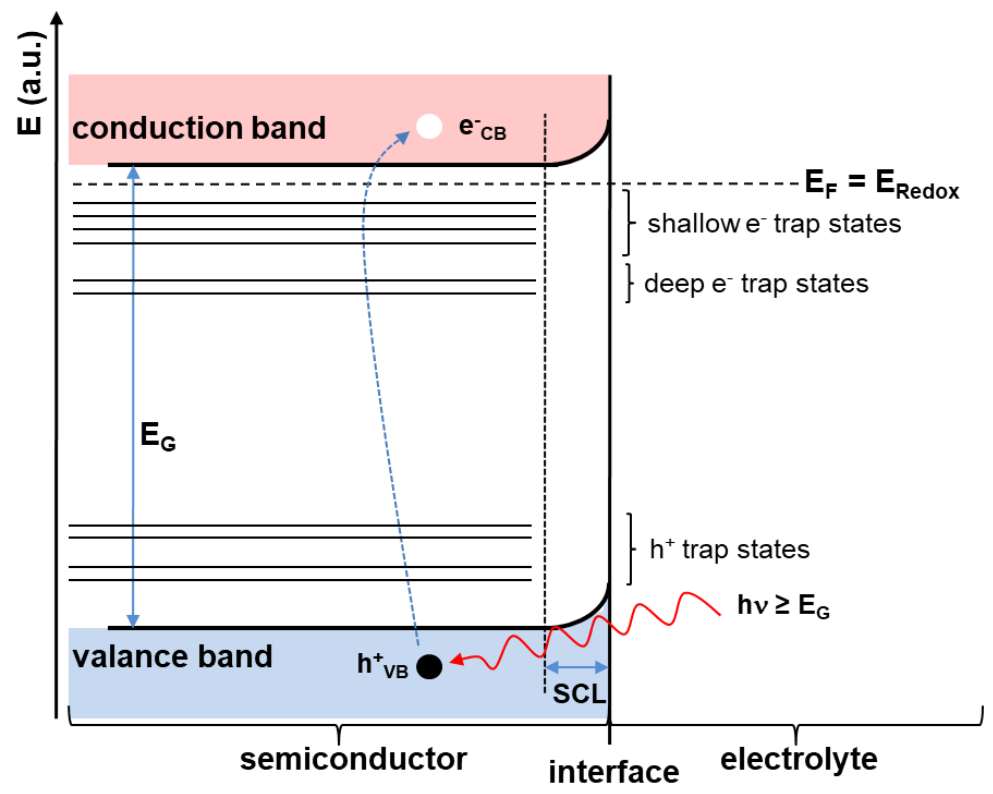

Figure 1: Schematic representation of $\mathrm{TiO}_{2}$ energy levels that is brought into contact with a certain type of aqueous redox system. Denotations are the following:

$\mathrm{e}^{-}{ }_{\mathrm{CB}}$ : conduction band electron

$\mathrm{h}^{+}{ }_{\mathrm{VB}}$ : valance band hole

$\mathrm{E}_{\mathrm{G}}$ : bandgap energy

$\mathrm{E}_{\mathrm{F}}$ : Fermi energy level

SCL: space-charge layer

$E_{\text {Redox }}$ : redox potential of the adjoining aqueous electrolyte

\subsection{Parameters affecting charge recombination}

Concerning incident photon flux, there is a threshold above which recombination becomes dominant [63-65]. It is supposed, that below this critical limit, excitons are consumed more rapidly by redox reactions than by recombination. This behavior can be explained considering the charge carrier density within the semiconductor. When it is higher (at higher flux) there is a smaller initial distance between electrons and holes, therefore, the probability of recombination will increase. This statement is easy to concede by taking into account the enhanced coulombic interactions (both repulsive and attractive) of charge carriers as the distance is shortened between them. Similar conclusions have been drawn on the effect of particle size, based on model calculations. It is pointed out, that bigger particles support better spatial separation of carriers [54]. The model predicted an increase in quantum yield as the particle size increased from 3 to $21 \mathrm{~nm}$. Other authors argued for the importance of crystallite size-dependent surface/volume ratio, being determinative of exciton generation-consumption relation [66]. In connection with this, larger surface area (i.e., smaller particles) can usually be associated with more lattice defects acting as recombination centers [67-68]. These defects can be eliminated by inducing a lattice rearrangement via heating. Depending on the applied temperature and actual catalyst properties (porosity, phase composition), simultaneous 
increase of particle sizes and sintering may occur. Higher photocatalytic activity attributed to the removal of recombination centers was achieved upon thermal treatment under $\mathrm{H}_{2}$-containing atmosphere [69]. By this method, however, oxygen vacancies - often referred to as $\mathrm{Ti}^{3+}$ centers - are introduced into the crystal structure, both on surface and in subsurface regions [70]. Hole trapping function of these sites is presumed, which might also play a role in the enhanced activity observed for various substances [71]. Additionally, like already mentioned, surface located O-vacancies can be "healed" by water adsorption creating hydroxyl groups that are advantageous in photocatalytic processes. These defects, by the way, manifest themselves in a blueish color of $\mathrm{TiO}_{2}$ samples owing to their intra-bandgap states, which allow visible light excitation [70].

A basic requirement of the photocatalytic process is the presence of reactants with appropriate redox potentials compared to the valance and conduction band levels. Continuous reduction and oxidation is thereby ensured via electron transfer through the interface. The lack of suitable acceptor and donor type materials leads to charge accumulation within the semiconductor, therefore the probability of recombination increases. If oxidative destruction of a given parent/target compound (e.g., organic substrates) is aimed, simultaneous electron withdrawal has to occur at the catalyst surface. For this purpose, molecular oxygen is of particular importance among other frequently used electron scavenging materials, such as $\mathrm{H}_{2} \mathrm{O}_{2}$ [72-74], $\mathrm{Ag}^{+}$[72-73,75], etc. Oxygen is applicable in gas phase as well as in aqueous media, where it shows strong electrophilic character. Its adsorption is highly favored on irradiated $\mathrm{TiO}_{2}$ surfaces, outward electron transfer rate, therefore, may not be mass transport limited. As another advantage, oxygen forms reactive radical/radical ion species (see next chapter) which directly contribute to the photocatalytic decomposition process [63].

Surface OH-group density is also proven to have influence on charge separation through the ability of these species to trap both holes and electrons. Basically, two types are distinguished: bridging $\left(\mathrm{O}_{\mathrm{Br}} \mathrm{H}\right)$ and terminal $\left(\mathrm{O}_{\mathrm{T}} \mathrm{H}\right)$ hydroxyl groups. The former can actually be considered as adsorbed protons on the 2 -fold coordinated bridging oxygen atom ( $\left.\mathrm{Ti}-\mathrm{OH}^{+}-\mathrm{Ti}\right)$, while the latter is equally regarded as a hydroxide ion bound to the 5 -fold coordinated $\mathrm{Ti}^{4+}$ atoms or as the product of the dissociative adsorption of water at these sites [39]. In fact, the OH-group density is mainly determined by the crystal structure [76-79] of the catalyst and it can hardly be adjusted posteriorly without inducing changes in other properties. Later on, a detailed description on surface characteristics and their role in photocatalysis will be presented.

There is another possibility to suppress charge carrier recombination: modification of electronic properties of $\mathrm{TiO}_{2}$ by doping. In this respect, the use of transition metals is the most promising and therefore the most extensively studied field. Incorporation of these elements proved to have various effects on catalysts' behavior (such as adsorption capacity, charge transfer rate, surface hydrophilicity, etc.) eventually leading to an enhanced photocatalytic performance [80-82]. In some 
cases (for a few donor and acceptor type dopants, e.g., $\mathrm{Al}^{3+}, \mathrm{Cr}^{3+}, \mathrm{Ta}^{5+}$, etc.), the presence of heterocations was found to be detrimental, since they can act as recombination centers [83]. Improved charge carrier separation obtained by doping is explained in the following way. Incorporated ions narrow down the thickness of the space-charge layer proportionally to their concentration [84]. At optimal dopant loading, the width of the space-charge region approximates the penetration depth of the light thus ensuring the most efficient separation. Beyond this concentration limit the space-charge layer narrows further, so its thickness will be lower than light penetration depth. Consequently, hindering on recombination will decrease [85].

The main aim of $\mathrm{TiO}_{2}$ doping is often the achievement of bathochromic shift, which means the extension of excitability towards the visible region of light. This was successfully implemented using numerous transition metals and non-metallic elements [1]. The possibility of lower energy excitation is created by intra-bandgap states introduced through incorporation of dopant elements into the lattice structure.

\subsection{Interfacial charge transfer and subsequent surface/near-surface reactions in aqueous phase}

From this stage of heterogeneous photocatalytic processes, some major differences will arise between gas and liquid phase reactions. This fact seems to be obvious if one considers the essential differences in mass and heat transport, adsorption, etc. In the following treatments, we would like to focus mostly on liquid phase-related phenomena. The main reason of doing so is that in our studies, special emphasis has been laid on the interpretation of these reactions. Although gas phase experiments have also been made for a few samples, the majority of photocatalytic tests were performed in aqueous suspensions. These results are, in addition, supplemented by other aqueous phase experiments, like $\mathrm{O}_{2}$-consumption and dynamic light scattering (DLS) measurements.

Separated charge carriers produced upon excitation migrate inside the $\mathrm{TiO}_{2}$ structure. A given portion of them disappears due to recombination and their energy dissipates as lattice vibrations. The rest of them successfully reaches the catalyst surface and initiate chemical reactions. The way of charge transfer through the phase boundary is still disputed to some extent. Some authors claim, that the mechanism must include charge trapping (see Chapter 2.2), while others argue for the possibility of charge carrier transfer either from free state [86].

The rate of the photocatalytic process is greatly affected by adsorption characteristics, especially if mass transport limitations are assumed to be negligible (the case of ideally homogeneous system produced by perfect mixing) [87]. For aerated aqueous solutions, kinetics usually obeys the Langmuir-Hinshelwood law [88]. It takes into account the surface coverage of the primary charge 
acceptors, as written below:

$$
\mathrm{r}_{0}=k \Theta_{O 2} \Theta_{S}
$$

According to this theory, the initial reaction rate $\left(\mathrm{r}_{0}\right)$ is directly proportional to the coverage of both the $\mathrm{O}_{2}$ and the substrate $\left(\Theta_{\mathrm{O} 2}\right.$ and $\Theta_{\mathrm{S}}$, respectively) and to the reaction rate coefficient $(\mathrm{k})$. Oxygen usually serves as an efficient electron scavenger; its presence allows the model compound (or other substances, e.g., solvent molecules) to be oxidized by the holes. The coverage values can be expressed as:

$$
\Theta_{O 2, S}=\frac{K_{O 2, S} c_{O 2, S}}{1+K_{O 2, S} c_{O 2, S}}
$$

where elements $K$ and $c$ are the corresponding adsorption coefficients and equilibrium concentrations, respectively. For ensuring the validity of Equation 6, some assumptions have to be made [89]:

a) oxygen is adsorbed molecularly and not in dissociated form

b) the substrate and the oxygen molecules are bound to different sites, i.e., there is no competition between them

c) intermediates formed during the reaction do not influence the adsorption of the above reactants (due to their low concentrations or to the lack of specific adsorption).

If a considerable competition takes place between the substrate and other molecules (such as intermediates, solvent molecules, etc.), Equation 6 must be written in a more general form:

$$
\Theta_{S}=\frac{K_{S} c_{S}}{1+K_{S} c_{S}+\sum_{i=1}^{n} K_{i} c_{i}}
$$

Here, $i$ is equal to the number of competing substances and $K_{i}$ and $c_{i}$ are the corresponding adsorption coefficients and concentrations, respectively.

There are other cases as well, when the Langmuir-Hinshelwood law is not suitable for describing the experimental findings. For those, often Freundlich's adsorption model is used. Its theory proved to be not always valid, nevertheless, it gives acceptable description on the adsorption behavior of several molecules [90]. The Freundlich-type relation reads as follows:

$$
r_{0}=k K_{F} c_{0}^{n}
$$

where all elements are with the same meaning as previously, while exponent $n$ is characteristic of the strength of adsorption. 
Besides these, it is important to introduce other kinetic approaches treating photocatalytic degradation as a process that is independent of adsorption. It is particularly reasonable for those target compounds, which display no significant/measurable adsorption on $\mathrm{TiO}_{2}$ surface. Here, chemical transformations proceed via indirect charge transfer, assisted by radicals produced on irradiated titania surfaces. The decomposition rate of non-adsorbing organic molecules - for instance phenol, which was used for aqueous phase photocatalytic tests in our work - is therefore determined only by the rate of radical production and by the kinetics of the radical-compound reaction itself. If a perfectly mixed system is presumed and other parameters - such as light intensity, oxygen feed rate, temperature - are kept constant, the reaction will most probably provide a constant radical production. In this case, the situation is still not simply about the homogeneous interaction between free radicals and molecules, since adsorbed radical species may also be regarded as potential reactants. By the same token, resulting intermediates and inorganic end-products can further complicate the kinetic picture through being adsorbates, reactants and/or having influence on the ionic strength and $\mathrm{pH}$.

From the above explanation it may be concluded, that obtaining valid kinetic information on heterogeneous photocatalytic reactions is rather difficult. Concerning our studies, only the comparison of photocatalytic activities, and not a comprehensive mechanistic study was aimed at. Especially as latter had already been done by others for all model compounds used here (related papers will be referred later in the corresponding sections). Accordingly, we expressed photocatalytic activity as the initial decomposition rate of the target molecules, both in liquid and in gas phase experiments. This type of evaluation ensures a simplified treatment, with which perturbing effects of the multicomponent nature can be eliminated. At the very early stages of the reaction, namely, the system contains predominantly the target compound. Second order polynomial functions fitted on the experimental decomposition curves gave satisfactory description from mathematical point of view, since all regression coefficients exceeded 0.98. Initial breakdown rates were then obtained by calculating the slopes of empirical functions at $\mathrm{t}=0$.

High performance/pressure liquid chromatography (HPLC) and gas chromatography (GC) methods are suitable for gathering information on product distribution. Nevertheless, they do not tell much about the overall mineralization efficiency. For this purpose, total organic carbon (TOC) measurements are more adequate, as the exact carbon balance could be obtained for liquid phase reactions. Despite its usefulness of providing such essential information, we employed this technique relatively unfrequently. The reason is that the extremely clean circumstances and the complicated preparation and measurement methods make TOC more expensive and time consuming than chromatography. However, our findings revealed that for flame-made $\mathrm{TiO}_{2}$ samples, the initial rates of phenol decomposition correlates with TOC removal rates to an acceptable extent, so we regard chromatography equally suitable for the comparison of photocatalytic efficiencies. 
If molecular oxygen is adsorbed on excited titania surface, it behaves as an electron acceptor:

$$
\mathrm{e}^{-}+\mathrm{O}_{2} \rightarrow \mathrm{O}_{2}^{--}
$$

The resulting superoxide anion radical is known to react further according to the following scheme [61]:

$$
\mathrm{O}_{2}{ }^{-} \stackrel{H^{+}}{\longrightarrow} \mathrm{HO}_{2} \cdot \stackrel{e^{-}}{\longrightarrow} \mathrm{HO}_{2}^{-} \stackrel{H^{+}}{\longrightarrow} \mathrm{H}_{2} \mathrm{O}_{2} \stackrel{h v}{\longrightarrow} 2 \cdot \mathrm{OH}
$$

These oxygen-containing species are of great importance in photocatalytic destruction [91]. Each of them can interact with target molecules or intermediates due to their high reactivity. Oxygenated compounds thus formed are eventually transforming into $\mathrm{CO}_{2}, \mathrm{H}_{2} \mathrm{O}$ and other inorganic materials, resulting in the destruction of organic pollutants. There is an optimal radical-substrate ratio for achieving the highest possible activity. If this optimum is exceeded, recombination between radicals (e.g., Reaction 11) occurs to a greater extent, therefore the apparent rate of photocatalysis decreases.

$$
\mathrm{HO}_{2} \cdot+\cdot \mathrm{OH} \rightarrow \mathrm{H}_{2} \mathrm{O}+\mathrm{O}_{2}
$$

As it is seen from Scheme 10, even OH-radicals can be produced acting as reactants with the highest oxidation potential in these systems. Besides the release from surface-bound state [92], this is the other way of generating mobile $\mathrm{OH}$-radical. The existence of free radicals inside the bulk medium is required for the decomposition of those compounds, which are not able to adsorb on $\mathrm{TiO}_{2}$ surface in consequence of electrostatic repulsion or hydrophobic character. Mobile radicals have short diffusion length in water due to their high reactivity. This fact suggests that reactions involving radical species have to take place close to the semiconductor surface [93]. In this respect, the situation is a bit more advantageous in gas phase as having lower concentration of potential reaction partners. Under these circumstances, free radicals have successfully been detected in a relatively large distance from the immobilized catalyst [94]. Some authors, though, have disputed the possibility of such a long-range reactionless $\mathrm{OH}$-radical diffusion in air and offered a new mechanism for this so-called remote photocatalytic oxidation. They [95] postulated that these OH-radicals are created by UV-photolysis of $\mathrm{H}_{2} \mathrm{O}_{2}$ molecules, which species were previously produced on $\mathrm{TiO}_{2}$ particles and could travel a longer distance from the catalyst, owing to their lower reactivity.

In aqueous medium, formation of hydroxyl radicals is presumed mainly through direct electron transfer from adsorbed water molecules or hydroxide ions [39]:

$$
\mathrm{h}^{+}+\mathrm{H}_{2} \mathrm{O} \rightarrow \cdot \mathrm{OH}+\mathrm{H}^{+}
$$




$$
\mathrm{h}^{+}+\mathrm{OH}^{-} \rightarrow \cdot \mathrm{OH}
$$

A potential intermediate step of Reaction 12 has already been described by Reaction 3, where an OHgroup, previously formed in the interaction of a water molecule and an $\mathrm{O}$-vacancy, captures a hole and creates a surface-bound $\mathrm{OH}$-radical. There are basically two pathways for $\mathrm{OH}$-radicals to react with compounds: addition and hydrogen abstraction. For oxidation of substituted aromatic hydrocarbons, the former route plays an essential role [96], while for e.g., chlorinated ethanes the latter proved to be the most likely [97]. Naturally, when a given substrate tends to adsorb on titania, direct hole oxidation will prevail over the radical-mediated route. Hydrocarbon radicals $(\mathrm{R} \cdot)$ here produced can interact with oxygen or water molecules this way forming oxygenated compounds [98]. Although hydroxyl radical- and hole-catalyzed reactions are rather different, they often give very similar product distribution in aerated aqueous solutions making the distinction between the two difficult.

During photocatalytic destruction, alkyl chains, aldehydes and finally organic acids (more and more oxidized species) will appear as oxygenated intermediates of the process. In a subsequent step, acids undergo decarboxylation according to the following equations [75]:

$$
\begin{array}{r}
\mathrm{RCOOH} \rightarrow \mathrm{RH}+\mathrm{CO}_{2} \quad(14) \\
2 \mathrm{RCOOH} \rightarrow \mathrm{R}-\mathrm{R}+\mathrm{H}_{2}+2 \mathrm{CO}_{2}
\end{array}
$$

Reaction 15 shows analogy with the so-called Kolbe electrosynthesis, it is, therefore, generally referred to as photo-Kolbe reaction. By the above transformations, polluting hydrocarbons can be fragmented stepwise into chains with less and less carbon atoms, until their complete mineralization.

\subsection{Decomposition mechanisms of model compounds}

\subsubsection{Aqueous phase: phenol}

The reactivity of target compounds in $\mathrm{TiO}_{2}$-assisted photocatalysis can be classified by the aid of numerous experimental observations and theoretical considerations published in the literature. In general, oxygenated molecules are more readily degradable than pure hydrocarbons, and aromatic substances more readily than aliphatic ones under the same conditions [1]. Here, the role of chemical 
structure can be deduced from two reasons. It determines the electron density along the molecule as well as its hydrophilicity (although the two are strongly related to one another). The first one defines the position and the nature of the most reactive sites (e.g., against radicals), while hydrophilicity governs solvation and adsorption processes in aqueous reaction systems.

Phenol is known as a weakly adsorbing compound on $\mathrm{TiO}_{2}$ surfaces due to its hydrophobic nature. This was also confirmed in our studies, since we could not observe any sign of adsorption even in a suspension of four times higher $\mathrm{TiO}_{2}$ content than usually applied (for details, see Section 4.2.2). Under our experimental conditions, therefore, phenol is reasonably presumed to be oxidized by hydroxyl radicals rather than directly by photogenerated holes [99-100]. OH-radicals preferentially attack the aromatic ring (electrophilic aromatic substitution), particularly if it is activated by some electron donating substituents, such as OH-groups. Based on this feature, Hammett constants $(\sigma)$ of several aromatic compounds have been correlated with their reactivity [101], because it expresses the effect of different substituents on their electronic structure. The higher the electron density, the more likely the electrophilic attack occurs on a given site of the molecule.

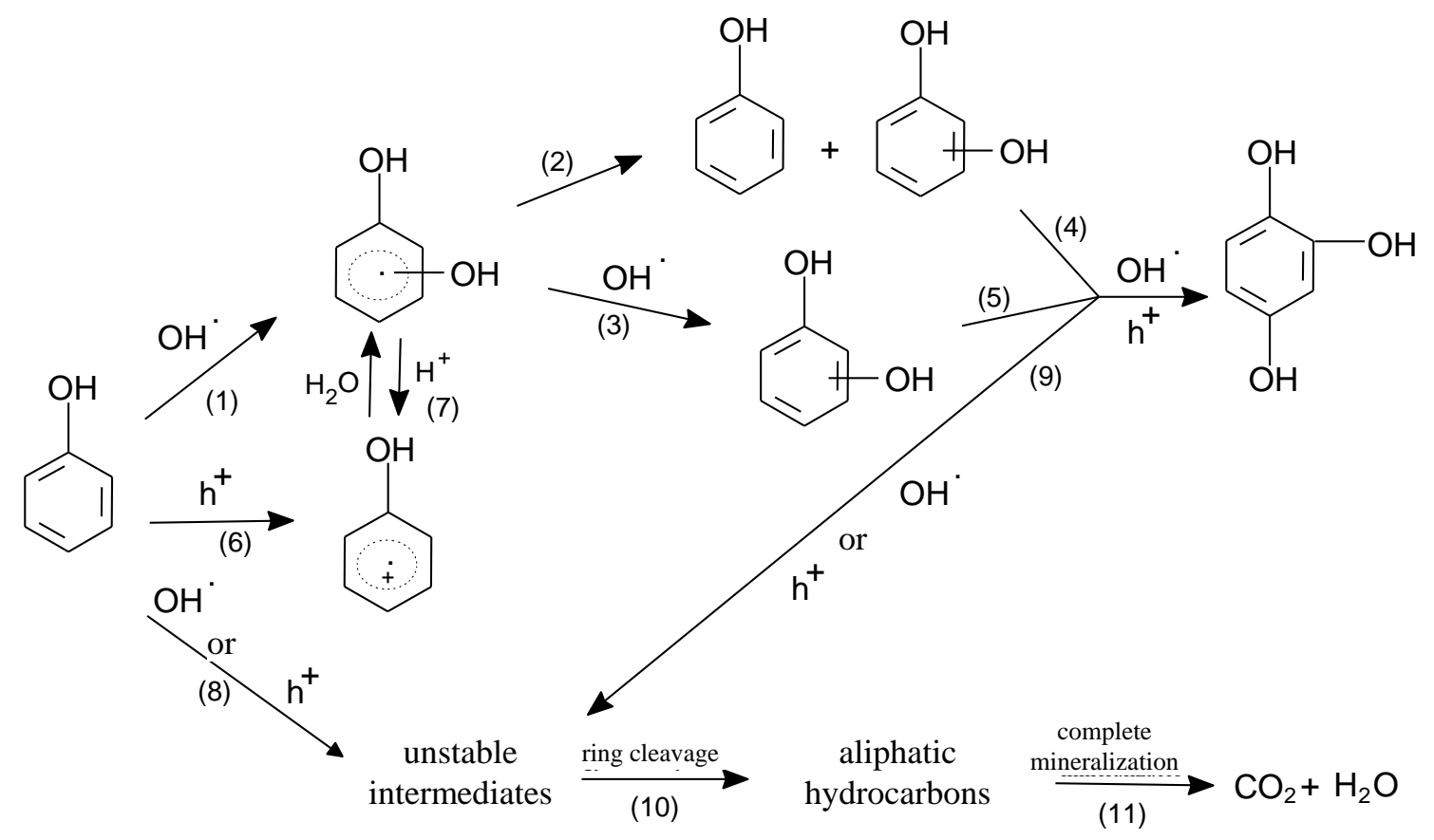

Figure 2: Summarized mechanism of phenol decomposition taken from [89]

As it is shown in the summing scheme of phenol's photocatalytic reaction pathways (Figure 2, taken from [89]), decomposition may start with OH-radical insertion (process 1) on the ring [84] producing the so-called dihydroxycyclohexadienyl radical (DHCHD). This compound can undergo disproportionation (2) yielding phenol and dihydroxybenzenes or subsequent oxidation by an additional OH-radical (3) that way providing dihydroxybenzenes as well. After this, another oxidation 
step will come, where two routes are possible: formation of either trihydroxy benzenes $(4,5)$ or extremely unstable intermediates (9), which otherwise were evidenced to form directly from phenol (8). Latter species has the tendency to decompose in ring cleavage reactions resulting in oxygenated aliphatic hydrocarbons (10, mostly organic acids and aldehydes) [73]. These molecules transform further in sequences of multiple decarboxylation and oxidation steps (11) as it was demonstrated in Reactions 14 and 15. It is worth remarking here, that the initial radical-assisted oxidation processes could equally be regarded as a consequence of direct charge transfer (6, through holes) since a very similar product distribution is expected for both pathways. This alternative degradation route was also evidenced, but only at roughly 4-times higher phenol-to- $\mathrm{TiO}_{2}$ ratio [102] than what we applied. This step yields phenoxyl radical first, which then reacts further with either oxygen or $\mathrm{HO}_{2} \cdot$ radical, giving ozonide type structures. Finally, an intramolecular rearrangement will result in ring opening products, similarly as above [73]. Adsorption strengths of the manifold intermediates essentially determine reaction kinetics. Although no comprehensive quantified comparative experimental results are published, relative adsorption abilities may be extrapolated from theoretical considerations (based on molecular structure) and from the individual reports including some information on product analytics. Like it was stated, primary intermediates of phenol are dihydroxybenzenes, namely pyrocatechol (1,2-; PC) and hydroquinone (1,4-; HQ). Out of these two substances, PC might adsorb more efficiently due to its ortho-situated two hydroxyl groups, possibly facilitating a stronger connection with $\mathrm{TiO}_{2}$ surfaces (similarly as at acetic and formic acid; see next section). This can be a potential reason why PC usually appears with - sometimes much - lower maximum liquid phase concentrations than HQ (see Chapter 5.9 and [102]), whereas HQ is supposed to decompose faster [103]. It seems, however, rather certain, that amongst the numerous ring opening products, shortchain organic acids (in particular, acetic and formic) have superior adsorption strength owing to their carboxyl functions. For instance, Araña and co-workers identified mostly formate species - apart from other carboxylic and hydroxylated benzoic acids - by IR(infrared)-spectroscopy on $\mathrm{TiO}_{2}$ particles, filtered off from aqueous phenolic reaction mixtures after being irradiated for different timespans [102]. Results of Teoh et al. also corroborate the favored adsorption of small carboxylic acids [104-105]. Documented examples exist in the case of acetaldehyde (next section), where these degradation products are able to displace the otherwise strongly bound parent substance from the catalyst surface. Clearly, the situation must be the same for methanol decomposition, at which formic acid also appears. Targeted investigations on adsorption strengths could not be found in the literature, nevertheless, some authors attribute fast $\mathrm{TiO}_{2}$ poisoning to the accumulating formates [106] that practically confirms the basic assumption. 


\subsubsection{Gas phase: methanol and acetaldehyde}

In order to obtain further information on the catalytic efficiency of our $\mathrm{TiO}_{2}$ samples, gas phase decomposition of methanol and acetaldehyde were also studied.

Methanol adsorbs dissociatively on $\mathrm{TiO}_{2}$ forming methoxy and hydroxyl groups [107-108]. Two distinct binding sites were proposed to be available for methoxy species: $\mathrm{Ti}^{4+}$ centers and anion vacancies, among which the latter type possesses higher affinity to methoxy groups [107]. Methanol degradation in gas phase is known to essentially be initiated by photogenerated holes producing formaldehyde, formic acid and finally $\mathrm{CO}_{2}$ [106]. In aqueous media, the presence of excess water can shift the oxidation pathway towards the radical route, because competitive adsorption takes place between water and methanol molecules [109-110]. In gas phase, the same phenomenon was observed by Wang et al. [111], but only when a relatively large amount of water was added to the system (water-to-methanol ratio was $c a$. 300). In this experiment, although the signals of surface-bound methoxy groups have disappeared due to water co-adsorption, physisorbed methanol remained detectable. As discussed earlier, displacement of methanol from $\mathrm{TiO}_{2}$ surfaces can be triggered by formic acid as well, whose strong adsorption could lead to catalyst deactivation under certain reaction conditions [106].

For acetaldehyde, two alternative pathways of adsorption were evidenced by IR-spectroscopy [112-113]. A given fraction of the molecules bonds to Lewis acid sites $\left(\mathrm{Ti}^{4+}, \mathrm{Ti}^{3+}\right)$ through oxygen lone pairs and others form H-bonds with surface hydroxyl groups. There are basically two possible initial steps of acetaldehyde decomposition. If it adsorbs on $\mathrm{Ti}^{3+}$ sites, the reaction proceeds through acetic acid and then formaldehyde appears. When acetaldehyde binds to $\mathrm{Ti}^{4+}$ centers, it will directly be transformed into formaldehyde without being oxidized to acetic acid. Subsequent steps are the same in both cases: formic acid and finally $\mathrm{CO}_{2}$ will be produced [114-115]. It was found, that direct hole-oxidation of acetaldehyde is not the only possible pathway, there may be a significant contribution of radical-mediated reactions, driven either by $\mathrm{OH}$ or by other oxygen-containing radicals. [114-116]. This is caused by the higher degree of interfacial accumulation of the particularly well-adsorbing intermediates. Formic and acetic acid can bind strongly to Lewis acid sites in uni- as well as in bidentate mode through the lone pairs of the two oxygen atoms [117-118]. At sufficiently high coverage, this strong interaction effectively hinders the adsorption of acetaldehyde [119-120], therefore oxidation via radicals starts to prevail. Water is proven to be an efficient competitor for $\mathrm{TiO}_{2}$ sites here as well [121], although the parent substance could not fully be expelled from the surface even having $34 \%$ of relative humidity $(\mathrm{RH})$ in the photoreactor. 


\subsection{Effects of process parameters in aqueous photocatalytic degradation}

Besides chemical quality of both the catalyst and the model compound, most process parameters greatly influence photocatalytic activities. It is to be noted, that every factors listed and analyzed below were carefully investigated in advance, and were kept constant (except $\mathrm{pH}$ ) throughout our studies. Effects of photon flux will not be treated in this chapter because it has already been described in details before.

\section{Catalyst loading}

In general, photocatalytic decomposition rate increases with catalyst content due to the larger active surface available for adsorption and light absorption. However, there is an optimum level of catalyst loading above which the breakdown starts slowing down. The reason lies in enhanced light scattering of the $\mathrm{TiO}_{2}$ particles, causing the reduction of optical penetration length in the dispersion [122]. It is hence an essential parameter to be optimized when designing a slurry photoreactor. Decreased light penetration can be compensated applying appropriate solution layer thickness and light intensity.

In reactors using immobilized $\mathrm{TiO}_{2}$, thickness and porosity of the catalyst film is of key importance. Comparing compact catalyst films to porous systems, the latter is more beneficial as having higher effective surface, which is, in turn, proportional to the film thickness. Nevertheless, thicker films are accompanied by the problem of higher mass transfer resistance inside the pores thus reducing the overall decomposition rate [1].

\section{Concentration of the substrate}

The variation of the initial photocatalytic degradation rate in the function of target substrate concentration goes into saturation [1]. The explanation is simple: beyond a certain concentration, either the number of available biding sites or the amount of charge carriers/radicals will be the restraint for any further increase of the reaction rate. 


\section{Temperature}

Slight changes in temperature are known to have only minor influence on the photocatalytic oxidation rate [84]. The low thermal energy in the near-room-temperature regime (used in our studies) has almost no contribution to the activation energy of $\mathrm{TiO}_{2}$, neither to relative Fermi level positions [123]. Interfacial electron transfer rate [124] and desorption processes [125] are reported to be a bit more sensitive to temperature variations, but these factors remain still negligible in the range applied $\left(25.0 \pm 0.1^{\circ} \mathrm{C}\right)$. Anyhow, using temperature control is always advisable in order to avoid aggregate effects. In our experiments, an accidental shutdown of the thermostat led to an uncontrolled warming up to $35-40^{\circ} \mathrm{C}$, which resulted in a significant change of the given sample's activity.

\section{Concentration of dissolved oxygen}

The key role of oxygen in $\mathrm{TiO}_{2}$-assisted photocatalysis has already been demonstrated: it is an effective electron scavenger and takes part in the formation of radical species. It was found to be essential for the decomposition of most organic compounds [126], while only in a few instances proved to have no or negligible effect on reaction rate (e.g., for trichloroethylene; [127]).

\section{Presence of inorganic ions}

Inorganic anions $\left(\mathrm{Cl}^{-}, \mathrm{ClO}_{4}{ }^{-}, \mathrm{NO}_{3}{ }^{-}, \mathrm{CO}_{3}{ }^{2-}, \mathrm{HCO}_{3}{ }^{-}, \mathrm{SO}_{4}{ }^{2-}, \mathrm{PO}_{4}{ }^{3-}\right)$ usually have an adverse effect on photocatalytic degradation rate through competing for radicals and/or blocking the active sites on $\mathrm{TiO}_{2}$ surfaces [128]. For example, in the case of phosphate adsorption, anchoring to the surface is so strong that only alkali washing can entirely remove these ions. Here, a considerable (almost 50\%) rate reduction was observed for simple organics (e.g., ethanol) with a phosphate concentration as low as $1 \mathrm{mM}$.

For inorganic cations both beneficial $\left(\mathrm{Fe}^{3+}, \mathrm{Ag}^{+}, \mathrm{Cu}^{2+}\right)$ and detrimental $\left(\mathrm{Cr}^{3+}\right)$ effects have been reported with strong dependence on chemical quality and concentration of the given ionic species. Cations often act as electron acceptors or provide $\mathrm{OH}$-radicals participating in Fenton-type reactions with $\mathrm{H}_{2} \mathrm{O}_{2}$ thus improving photocatalytic efficiency [129]. Their ability of complex formation with substrate molecules also plays a role in the degradation process. At lower concentrations, they can facilitate charge transfer from catalyst particles [130], however, higher complex content may absorb a significant UV intensity this way retarding photocatalysis. Other disadvantageous phenomena are 
the precipitation and deposition of metal hydroxides on $\mathrm{TiO}_{2}$ surface at higher $\mathrm{pH}$, causing a sort of site blocking.

In our reaction systems, the usage of analytical grade materials and careful execution of all necessary experimental steps (catalyst synthesis and cleaning, preparations, sampling) ensured the exclusion of all unwanted ionic contaminations. Naturally, carbonates were formed during photocatalytic runs in amounts proportional to the total mineralization rates.

$p H$

$\mathrm{pH}$ is a special parameter greatly influencing the overall photocatalytic efficiency in aqueous media [131]. It simultaneously determines the protonation state of the surface and of all acidic/basic chemicals being present in the system.

Amphoteric nature of $\mathrm{TiO}_{2}$ is well-known and has been characterized by many research groups, that sometimes assigned different mechanisms to the phenomenon. The interpretation of Kormann $e t$ al. [132] contains proton exchange reactions of "titanol" groups (Reaction 16), which - though it is not specified in the text - must mean the terminal hydroxyl groups themselves.

$$
>\mathrm{TiOH}_{2}^{+} \stackrel{\mathrm{pKa} 1}{\longleftrightarrow}>\mathrm{TiOH} \stackrel{\mathrm{pKa} 2}{\longleftrightarrow}>\mathrm{TiO}^{-}
$$

This can be suspected from the given values of microscopic dissociation constants $\left(\mathrm{pK}_{\mathrm{a} 1}=4.5 ; \mathrm{pK}_{\mathrm{a} 2}\right.$ $=8.0$ ), as similar $\mathrm{pK}_{\mathrm{a}}-\mathrm{S}$ of the respective equilibria also appear elsewhere in the literature [133-134]. The deprotonation of $\mathrm{O}_{\mathrm{Br}} \mathrm{H}^{+}$-groups is calculated to occur at a relatively low $\mathrm{pH}$ range $\left(\mathrm{pK}_{\mathrm{a}}=2.8\right)$, being ascribed to the weaker interaction between the proton and the bridging oxygen atom [133]. Note, that above values may show certain dependencies on the experimental circumstances (ionic strength), on catalyst qualities (phase composition, ratio of exposed facets) as well as on the analytical technique/computational method applied.

So, as demonstrated, the nature and the density of hydroxyl groups essentially control the sign and the extent of surface charges carried at a given $\mathrm{pH}$. Charged $\mathrm{TiO}_{2}$ particles are more hydrophilic and surrounded by an electrical double layer consisting of dissolved ionic species. The coulombic repulsion acting between these layers (supposing that charges have the same sign) ensures colloidal stability preventing particles from agglomeration. The magnitude of this stabilizing effect is proportional to the compactness of the interacting layers, which is, in turn, determined by the surface charge density and the ionic strength of the solution. This feature is quite important regarding catalytic 
efficiency, since higher degree of dispersion (i.e., non-agglomerated system) means a higher effective surface for adsorption and light absorption [135]. Referred repulsive force can be expressed in numerical terms by the "zeta $(\zeta)$ potential" (or electrokinetic potential). It gives the potential difference between bulk medium and a point at the outer interface of the double layer (at the so-called slipping/shear plane) that remains attached to the particle during its movement. In summary, systems with lower $\zeta$-potential tend to coagulate. In aqueous $\mathrm{TiO}_{2}$ dispersions, the $\zeta$-potential varies with $\mathrm{pH}$, and at a characteristic value, particles will no longer hold net charge and the dispersion coagulates. This $\mathrm{pH}$ is known as isoelectric point ( $\mathrm{pH}_{\mathrm{IEP}}$ ), or point of zero charge ( $\mathrm{pH}$ PZC $)$ if there is no charge at all on the surface. Further modification of the $\mathrm{pH}$ will lead to charge reversal making the colloid stabilized again. Surveying a considerably large pool of publications, Kosmulski reported an average pHIEP value of 5.6 for polycrystalline $\mathrm{TiO}_{2}$ samples (138 different qualities were listed) having various phase compositions [136]. Below this $\mathrm{pH}$, adsorption of anionic species (electron donors/acceptors, indifferent ions) is favored, while above that, cationic substances will be attracted by the surface.

$\mathrm{pH}$ is also responsible for adjusting the chemical form of both organic and inorganic $\mathrm{pH}$ sensitive components, including radical ions (Reaction 10) and pollutants (Figure 2) as well. Conclusively, it directly affects radical production, degradation mechanisms and adsorption processes. Additionally, it is obvious, that $\mathrm{pH}$ is gradually decreasing upon the appearance of acidic intermediates and $\mathrm{CO}_{2}$ throughout the degradation [137], inducing continuous changes in circumstances mentioned. An illustrative example of $\mathrm{pH}$-dependent events could be the case of dichloroacetic acid, where $\mathrm{pH}$ modification switches between uni- and bi-dentate surface coordination modes altering the way of activation and further degradation [138].

As seen again, it is nearly impossible to consider every single parameter in the evaluation of heterogeneous photocatalytic reactions, especially due to their continuous mutual action on each other. Approximate mechanistic description of catalytic process, therefore, requires rational simplifications.

\subsection{Comparison of gas and aqueous phase environmental $\mathrm{TiO}_{2}$ applications}

First of all, it is worth emphasizing the advantages and high potential of heterogeneous photocatalysis in environmental applications, comparing to other well-known and routinely used methods.

In wastewater treatment, the most wide-spread method is probably the chemical oxidation (chlorination, ozonation), which is, however, not suitable for the mineralization of all types of organic substances [139]. A typical drawback of chlorination - closely related to the previous one - is the 
formation of toxic/carcinogenic by-products, such as trihalomethanes [140]. Although ozonation does not produce such hazardous compounds (except bromate ions), it has only short range and term effect hence requiring longer on-site generation to decontaminate larger volumes of water. Treatment time is, in turn, a crucial economic factor as it may considerably increase the amount of electricity consumed.

Activated sludge process (using microorganisms) is a preferred way of cleaning wastewaters due to the low cost and to the chemicals-free nature of this treatment. However, its application has significant limitations by some disadvantageous properties, for instance: low reaction rates, inefficiency at low pollutant level (ppb) and disposal of sludge. Furthermore, temperature and $\mathrm{pH}$ needs to be controlled in order to ensure ideal conditions for bacteria [1].

Adsorption technologies are often used as well, principally the method based on activated carbon. Despite that it is usually found powerful in the removal of organic pollutants from water, there are several problems to face with by its application. Activated carbon adsorbs most organic chemicals in a non-specific way, which leads to a fast decrease in its capacity, especially for those materials present in low concentration (e.g., extremely toxic compounds) [141]. Other drawbacks are the expensive regeneration (thermal desorption) and the necessity of decomposing/treating the pollutants released.

$\mathrm{TiO}_{2}$-assisted photocatalysis, as a part of advanced oxidation technologies (AOTs), have many advantages over conventional wastewater treatment techniques [1]. It avoids the use of additional chemicals (only $\mathrm{O}_{2}$ is needed as oxidant) and reactions mostly proceed up to total mineralization without yielding hazardous by-products. It provides rapid destruction under normal circumstances (temperature, pressure) and it is capable of catalyzing both reductive and oxidative transformations. Not to mention, that photocatalysis is applicable for removing low levels of contaminations (ppb range) even for such chemically inert compounds, like linear alkanes and their derivatives [75]. Nevertheless, there are still several improvements what need to be done in order to make heterogeneous photocatalysis a more powerful process. It has to be more efficient in utilizing both UV and visible light. On the other hand, it is important to solve the problem of catalyst recovery. $\mathrm{TiO}_{2}$ nanoparticles are difficult (i.e., not cost-efficient) to be filtered off, therefore the only reasonable way may be their immobilization.

Gas phase applications of $\mathrm{TiO}_{2}$ have already been implemented in industrial as well as in domestic environment. A wide variety of $\mathrm{TiO}_{2}$-based air-cleaning devices is now available in the market. This success is due to its highly beneficial properties and low costs comparing to other air treatment processes (adsorption, catalytic and thermal incineration). Photocatalytic reactors can be easily integrated into HVAC (heating, ventilation, air conditioning) systems and are operating under normal circumstances [17]. Additionally, the overall degradation of nitrogen and sulfur oxides 
together with volatile organic compounds (VOCs, the majority of hazardous air pollutants) is achievable [142] in most cases.

Contrasting photocatalytic air treatment with aqueous phase application, the former exhibits more advantages. In gas phase, a much lower UV intensity is required in lack of scattering and absorption losses caused by dispersed particles and the medium, respectively. Moreover, diffusion of reactants and products are less hindered, and $\mathrm{OH}$-radical scavenging species (such as $\mathrm{Cl}^{-}$) found in solutions are typically not present here. As an aggregate effect of all these factors, gas phase oxidation often displays even tenfold higher quantum efficiencies (that is, the ratio of one-electron chemical transformations per the number of photons absorbed) than those obtained in aqueous photocatalytic experiments for the same organic substrates [143].

Certainly, a few limitations also exist here [144]. When larger amount of pollutants is to be degraded, deactivation of the catalyst may occur owing to the surface accumulation of intermediates and by-products. In aqueous phase, the removal/dissolution of these substances proceeds continuously under optimal conditions, while in gas phase their adsorption is favored in lack of solvation effects. Accumulation may reach an extent causing reversible or irreversible deactivation [145-146], depending on the nature of compounds adsorbed. There are some possibilities to get rid of these accumulations and deposits blocking the active sites. Thermal regeneration is reported to be a rapid treatment, but sometimes it requires too high temperatures for effective cleaning [147]. The most obvious - though a bit more time-consuming - solution is photocatalytic regeneration, as it only needs a clean, dry or humidified airflow and simultaneous UV illumination [147-148]. This method is quite practical, since heating, addition of other chemicals or removal of catalyst bed can be avoided. Therefore, it may suitably be used e.g., in HVAC-integrated photocatalytic systems where disassembly and procedures previously described can hardly be carried out. In some special cases, however, a more drastic action is needed for regeneration: e.g., deposits of silicon-containing species can only be removed by alkaline washing [149].

Besides catalyst regeneration, water content of gas phase reaction environment strongly influences catalytic efficiency [110,142]. Its effect depends not only on its concentration, but the type of contaminations and the parameters applied as well. The presence of water is essential for the replenishment of $\mathrm{OH}$-radicals consumed during photocatalytic oxidation. If reaction rate is maintained at a relatively high level by the aid of sufficient amount of water, there is less chance of by-product accumulation and/or deposit formation [150]. At the same time, water adsorption can induce the displacement of adsorbed compounds if bonding stronger to titania surface, like it was detected for methanol [110]. Consequently, an optimal gas phase water concentration must exist at which a maximum reachable catalytic rate is assured by providing sufficient amount of radicals, preventing catalyst deactivation, but still leaving enough free active sites for molecules whose 
decomposition is faster if governed by direct charge transfer from the $\mathrm{TiO}_{2}$ particles. It is also important to consider, that physisorbed water may play a role in the adsorption of different substrates through solvation interactions [148].

\subsection{The role of structural properties of $\mathrm{TiO}_{2}$ in photocatalytic activity}

Three elements are needed to be considered here: particle size, specific surface area (SSA) and phase composition. The first two is closely connected being inverse proportionality between them (specified by shape-dependent functions) in case of non-porous catalyst particles. Surface area has basically dual effect on photocatalytic efficiency. The larger it is, the bigger amount of adsorbates can be bound on the catalyst. On the other hand, increasing the active surface leads to worsening of charge separation efficiency, as it was presented in Section 2.3. This interrelation creates the challenge to optimize the surface area/particle size specifically of the model compounds and of the media applied. It should be remarked that bandgap width (i.e., absorption threshold) is particle sizedependent; diameter decrease will be accompanied by bandgap widening. For instance, in the size range of 5-10 nm, a blue shift of $0.2 \mathrm{eV}$ has been observed [151].

Phase composition is also a determining structural feature in photocatalysis. Generally, there are two main crystalline phases reported to appear, irrespective of the synthesis method: anatase and rutile. As a third phase, brookite is only seldom present in nanosized $\mathrm{TiO}_{2}$ powders due to thermodynamic reasons. Its photocatalytic efficiency may still be regarded questionable, especially as it exists almost solely together with other titania phases [152-153]. Anyhow, some scientists evidenced the superior activity of bare brookite nanorods in $\mathrm{H}_{2}$ evolution experiments [154].

From the two most frequently found phases, rutile is thermodynamically more stable at all temperatures. Transformation of anatase to rutile, however, has kinetic hindrance under normal circumstances. It is so slow, that it practically does not occur. A significant acceleration can be observed for bulk $\mathrm{TiO}_{2}$ upon increasing the temperature above $600^{\circ} \mathrm{C}$. There are other factors as well, affecting the rate of transformation. Particles with different diameters have different surface free energy, which is determinative of thermodynamic conditions. Due to this phenomenon, the rutile form is stable at sizes greater than $35 \mathrm{~nm}[38,155]$. Reaching this threshold, a measurable rate can be achieved even at a temperature close to $400^{\circ} \mathrm{C}$ [156]. Moreover, surface and lattice defects have also been justified to vary (accelerate or inhibit) phase transformation. Here, the concentration of incorporated ionic impurities (dopants) plays key role, since they usually contribute to the formation of defect sites [156]. 
For most reactions, anatase is known to have much higher photocatalytic activity than rutile. This behavior is usually explained by the slightly higher Fermi level, higher surface $\mathrm{OH}$-group density [67] and lower charge recombination rates [157]. More recently, the superior activity of anatase was attributed to the tendency of some of its oxygen vacancies to lie subsurface without being quenched by adsorbates [158]. There are only a few instances, when rutile phase showed the same or higher activity than anatase [159]. Some authors suggest that catalysts containing 70-75\% anatase and 25$30 \%$ rutile exhibit the best performance (better than that of pure anatase) because of some synergetic effects between phases. EPR (electron paramagnetic resonance) measurements indicated spontaneous electron transfer at rutile-anatase interfaces, which results in better charge separation and thus in lower recombination rate [160-161]. The synergism described, however, has a prerequisite of chemically connected phases in order to enable interparticle or intercluster charge transfer [70]. This means, that merely physical contacts - existing e.g., between coagulated particles - are not likely to provide such a capability. From this aspect, there are several experimentally supported theories in the literature arguing for and against [162] this phenomenon. The majority of these studies have been made on the well-known $\mathrm{P} 25 \mathrm{TiO}_{2}$, one of the best photocatalysts in the market. We also used that as a reference material for the comparison of photocatalytic activities. During our investigations, incidentally, two different batches of P25 were identified with different rutile contents of 11 and 21 weight percent (named P25-B and P25-A, respectively). Based on our data, nevertheless, we can confirm that there is no unambiguous evidence of such a synergism as we could not find a clear correlation between activities and phase composition (see results later).

Apart from its questionable influence on charge separation, the presence of rutile could have only one benefit. This $\mathrm{TiO}_{2}$ phase has narrower bandgap $(3.02 \mathrm{eV})$ than that of anatase $(3.20 \mathrm{eV})$ allowing visible light excitation up to the wavelength of $410 \mathrm{~nm}$ [163]. If extended excitability was supported by better charge separation according to the above theory, it would lead to detectable catalytic performance enhancement when having higher rutile content in the samples. As it does not seem to be the case, we reasonably assume that rutile's photocatalytic activity is considerably lower than that of the anatase phase, indeed.

\subsection{Alternatives of photocatalytically active $\mathrm{TiO}_{2}$ synthesis}

In this chapter we will present some synthesis methods primarily focusing on the most preferred ones, but we briefly mention others with lower significance as well. Basically, they will be divided into two categories: $\mathrm{TiO}_{2}$ production from liquid and from gas phase. 


\subsubsection{Liquid phase routes}

Liquid phase routes are probably the most frequently used techniques for the production of titania photocatalysts. They are applicable to obtain thin films as well as nanopowders with controlled structural characteristics and particle morphology. Another beneficial property is the ease of producing homogeneously doped or composite catalyst systems. Their use is, however, limited by some important disadvantages: expensive precursors, long processing times and carbonaceous impurities. The three main procedures will now be simultaneously treated as they are strongly connected to one another and can also have several common steps and features. These routes are the following: co-precipitation, sol-gel and hydro(solvo)thermal synthesis. The first two give amorphous or partially crystallized titania (usually a mixture of titanium oxide/hydroxide with diverse stoichiometry) which creates the need of subsequent thermal treatment in order to yield photocatalytically active crystalline $\mathrm{TiO}_{2}$ phases.

Precipitation applies the addition of aqueous basic solutions (of $\mathrm{NaOH}, \mathrm{NH}_{4} \mathrm{OH}$, etc.) to Tiprecursors, mostly to $\mathrm{TiCl}_{3}$ [153] and $\mathrm{TiCl}_{4}$ [164]. $\mathrm{TiCl}_{4}$ is very hard to handle since it strongly hydrolyzes even in the presence of ambient air humidity. That is why it requires special circumstances for keeping the synthesis well-controlled. Adjusting particle size and size distribution is problematic anyway due to the rapid hydrolysis typically taking place.

The sol-gel route shows more favorable attributes than co-precipitation with respect to flexibility, purity and controllability. Basically, two types of starting materials are used: inorganic salts [165] and Ti-alkoxides. From the two, the alkoxide-based synthesis is more widely studied and employed as it provides numerous opportunities to fine-tune the synthesis procedure and therefore the properties of catalysts formed. Here, the initial sol gradually evolves into a gel structure through hydrolysis and condensation steps. The resulting disperse system contains solid state material in various morphologies, ranging from colloidal particles to continuous polymer networks [166]. Varying the temperature, the sol stability and the rates of either hydrolysis or condensation leads to controlled particle formation. These modifications can be achieved by the aid of pH-affecting [166], complexing/chelating agents [167] and using special alkoxides [168].

As it was stated earlier, post-synthetic thermal treatment (calcination) applied subsequent to the previous processes is often necessary, because otherwise samples will not have suitably high crystallinity and therefore photocatalytic activity. Heating $\mathrm{TiO}_{2}$ is, however, inevitably accompanied by some often unwanted changes, such as: reduction of surface area, phase transformation and modification of the surface chemical composition (loss of hydroxyl groups). Applying solvothermal technique for this purpose can eliminate a part of these problems. It involves the treatment of $\mathrm{TiO}_{\mathrm{x}} \mathrm{H}_{\mathrm{y}}$ in a certain medium (organic solvent or water) under self-produced pressures and temperatures 
usually not more than $250^{\circ} \mathrm{C}$. Due to the lower temperature a higher surface area can be preserved. Nevertheless, this method is also capable of controlling almost all the structural characteristics through regulating reaction parameters (solution composition, temperature, pressure, ageing time). Moreover, besides amorphous titania [169], many kinds of other titanium sources can be used, such as $\mathrm{TiOSO}_{4}$ [170], $\mathrm{TiCl}_{4}$ in acidic solution [171], etc.

There are a few more solution-based procedures which will be here mentioned but not intended to be discussed in details, partially because their relevance is limited either by technical difficulties and special requirements, or by poor productivity.

The microemulsion method uses water in oil dispersion (stabilized by surfactants) in order to ensure controlled particle growth, but it often encounters unwanted flocculation or aggregation [172]. Electrochemical synthesis is a suitable technique (exclusively) for producing advanced thin films with diverse characteristics. Problems of spontaneous hydrolysis can be overcome by applying both acidic and oxygen free environment [173] or organic medium [174]. Highly crystalline photocatalysts with large surface area have been produced by combustion synthesis, where a precursor-containing solution is rapidly heated up. Short dwell time (a few minutes) at higher temperatures $\left(600-700^{\circ} \mathrm{C}\right)$ yields a higher degree of crystallization but, at the same time, it hinders particle growth and phase transition from anatase to rutile [175].

\subsubsection{Gas phase methods}

Generally, gas phase synthesis routes offer a more expedient way of manufacturing solid state photocatalysts. They are capable of continuous operation, easily upscalable and do not require postsynthetic treatment. So, instead of multi-step liquid phase methods, easier and faster one-step synthesis is possible.

Chemical vapor deposition (CVD) enables fast formation of high quality films and polycrystalline materials even on industrial scale. The wide range of available precursors and producible compounds (metals, composite oxides, etc.) reflects its versatility well. CVD is based on the chemical reaction or decomposition of precursor vapors. Under ideal conditions, this chemical transformation takes place only at substrate-gas interfaces. Uncontrolled gas phase crystallization can have detrimental effect on yield, size distribution and morphology [176].

Physical vapor deposition (PVD) differs from CVD in many respects. First of all, chemical reaction does not take place in this case. At most, some minor changes in stoichiometry are possible when compounds (e.g., $\mathrm{TiO}_{2}$ ) and not pure elements are applied. Accordingly, usage of materials that remain stable in the gas phase is a primary prerequisite of the method. Solid state sources are 
converted into gaseous components by thermal or electron beam induced evaporation/sublimation. Here, reduced pressure is always desirable (as in some special CVD processes) in order to minimize gas phase collisions thus ensuring the uniformity and purity of the deposited film. In general, PVD produces films with properties (smoothness, thickness, crystallinity) superior to CVD-made ones, however, it is less productive and not efficient for coating larger surfaces. What is common for both techniques is that product quality is influenced by the following factors: applied pressure, gas phase composition, substrate temperature [177].

Despite it uses an aerosol of a given precursor solution, the so-called spray pyrolysis is also labeled as a CVD process. In fact, it is a sort of hybrid technology since crystallization can occur either from gas or liquid phase depending on the experimental setup and hence the time available for evaporation of the droplets. Spray pyrolysis involves the reaction of the precursor spray on a heated substrate (e.g., wall of a tubular furnace). The spray is generated by an atomizer/nozzle focused directly onto the high temperature surface. For providing atomization and further transfer of the aerosol, a pressurized gas stream (air, $\mathrm{N}_{2}, \mathrm{Ar}$, etc.) is used. Characteristics of the resulting films and nanoparticles are affected by the composition and concentration of the precursor solution, substratenozzle distance, gas flow rate and substrate temperature. In comparison to the foregoing processes, spray pyrolysis exhibits some important benefits: simplicity, low costs and high reproducibility [178].

Another spray-based CVD procedure is the aerosol flame synthesis (AFS) or, as often called, flame process. It has all the advantages listed for spray pyrolysis, moreover, very high production rates can be achieved here (even ca. $25 \mathrm{t} / \mathrm{h}$; [179]). AFS applies a given type of flame (several geometries and compositions are possible), in which a precursor is combusted. That means a series of chemical reactions, such as hydrolysis, oxidation/reduction, and crystallization. These reactions occur in a very short time (typically on millisecond scale; [180]) when the material is going through the hot zone of the flame. Beyond advantages mentioned above, smaller particles, uniform size distribution and higher activities are usually reported for AFS-made catalysts than for those synthesized by conventional spray pyrolysis [181-182]. Structural and chemical characteristics of the product can be fine-tuned readily by adjusting the main synthesis parameters (flame temperature, residence time). It is also to be noted, that AFS enables one-step synthesis of composite catalyst systems $\left(\mathrm{Pt} / \mathrm{TiO}_{2} ;[183]\right)$ or complex oxides such as $\mathrm{YBa}_{2} \mathrm{Cu}_{3} \mathrm{O}_{7}$ [184], simply by introducing the aqueous solution of the corresponding salts into the flame.

AFS has different subtypes classified by that precursor state in which it enters the flame. Accordingly, vapor-fed (VAFS) and liquid-fed AFS (LAFS) are known. In the latter case, an aerosol is generated via atomization from a liquid precursor. If the liquid is combustible and provides at least $50 \%$ of the energy liberated during combustion, it is called flame spray pyrolysis (FSP). When this 
requirement is not fulfilled (e.g., for water based solutions), then we speak about flame-assisted spray pyrolysis (FASP) [185].

In Table 1, we compiled an overview from the most notable and most referred papers dealing with nanosized $\mathrm{TiO}_{2}$ synthesis by means of AFS. It includes descriptions on the main parameters and some remarks concerning sample properties. It is seen, that different hydrocarbons (mostly $\left.\mathrm{CH}_{4}\right)$ are often used as fuels for flames [187-188], as well as hydrogen [199], while pure oxygen or air serves as oxidant. 


\begin{tabular}{|c|c|c|c|c|c|}
\hline Source & Fuel/oxidant & $\overline{\text { Precursor }}$ & $\begin{array}{l}\text { Burner and flame } \\
\text { description }\end{array}$ & $\begin{array}{l}\text { AFS } \\
\text { type }\end{array}$ & Highlights \\
\hline [179] & - & - & - & - & review \\
\hline [185] & - & - & - & - & review \\
\hline [186] & - & - & - & - & review \\
\hline [187] & $\mathrm{CH}_{4} / \mathrm{O}_{2}$ & $\begin{array}{l}\text { TTIP/xylene/ } \\
\text { acetonitrile mixture } \\
\text { (volume ratio: } \\
20: 55: 25) \\
+\mathrm{Pt}(\mathrm{acac})_{2}\end{array}$ & $\begin{array}{ll}- & \text { premixed flame } \\
\text { - } & \text { burner material: steel } \\
& \text { (presumably) } \\
\text { - } & \text { sheathing and } \\
& \text { atomization: } \mathrm{O}_{2}\end{array}$ & FSP & $\begin{array}{ll}- & \text { synthesis of pure and } \mathrm{Pt} / \mathrm{TiO}_{2} \\
- & \text { photocatalytic test reaction: } \\
\text { - } & \text { sucrose mineralization } \\
\text { - } & \text { better performance than } \mathrm{P} 25 \\
& \text { mostly spherical particles and } \\
\text { a bit of polyhedral }\end{array}$ \\
\hline$[188]$ & $\mathrm{CH}_{4} / \mathrm{O}_{2}$ & $\begin{array}{l}\text { TTIP } \\
\left(\mathrm{N}_{2} \text { as carrier gas }\right)\end{array}$ & $\begin{array}{ll}- & \text { co-flow diffusion flame } \\
\text { - } & \text { burner material: } \mathrm{n} / \mathrm{a}\end{array}$ & VAFS & $\begin{array}{ll}\text { - } & \text { photocatalytic test reaction: } \\
\text { methylene blue } \\
\text { decomposition } \\
\text { - } & \text { exclusively spherical } \\
\text { - } & \text { birticles } \\
\text { bimodal size distribution }\end{array}$ \\
\hline [189] & $\mathrm{CH}_{4} / \mathrm{O}_{2}$ & $\begin{array}{l}\text { TTIP } \\
\left(\mathrm{N}_{2} \text { as carrier gas }\right)\end{array}$ & $\begin{array}{ll}- & \text { co-flow diffusion flame } \\
\text { - } & \text { burner material: } \mathrm{n} / \mathrm{a}\end{array}$ & VAFS & $\begin{array}{ll}\text { - } & \text { spherical and smaller faceted } \\
\text { particles }\end{array}$ \\
\hline$[190]$ & $\mathrm{CH}_{4} / \mathrm{O}_{2}$ & $\begin{array}{l}\text { TTIP mixed with } \\
\text { xylene/acetonitrile } \\
\text { mixture } \\
\text { (volume ratio: } \\
11: 5 \text { ) }\end{array}$ & $\begin{array}{ll}- & \text { premixed flame } \\
- & \text { burner material: } \mathrm{n} / \mathrm{a} \\
- & \text { atomization: } \mathrm{O}_{2}\end{array}$ & FSP & $\begin{array}{l}\text { larger non-spherical and } \\
\text { smaller spherical/faceted } \\
\text { particles were separately } \\
\text { produced }\end{array}$ \\
\hline [191] & $\mathrm{C}_{2} \mathrm{H}_{4} / \mathrm{O}_{2}$ & $\begin{array}{l}\text { TTIP } \\
\text { (Ar as carrier gas) }\end{array}$ & $\begin{array}{ll} & \text { laminar flow flame } \\
\text { - } & \text { burner material: } \mathrm{n} / \mathrm{a}\end{array}$ & VAFS & $\begin{array}{ll}- & \text { highly aggregated polyhedral } \\
\text { and/or irregular-shaped } \\
\text { nanoparticles } \\
\text { - } \quad \mathrm{d} \approx 4.5 \mathrm{~nm}\end{array}$ \\
\hline [192] & $\mathrm{H}_{2} / \mathrm{O}_{2}$ & $\begin{array}{l}\mathrm{TiCl}_{4} \\
\left(\mathrm{~N}_{2} \text { as carrier gas }\right)\end{array}$ & $\begin{array}{ll} & \text { co-flow diffusion flame } \\
\text { - } & \text { burner material: stainless } \\
\text { steel }\end{array}$ & VAFS & $\begin{array}{ll}- & \text { mostly polyhedral particles } \\
\text { (only anatase) } \\
\text { - } \\
\text { photocatalytic test reaction: } \\
\text { Rhodamine B decomposition }\end{array}$ \\
\hline [193] & $\mathrm{C}_{2} \mathrm{H}_{4} / \mathrm{O}_{2}$ & $\begin{array}{l}\text { TTIP } \\
\text { (Ar as carrier gas) }\end{array}$ & $\begin{array}{ll}\text { - } & \text { stationary, premixed } \\
\text { planar flame (stagnation } \\
\text { flow) } \\
\text { - } & \text { burner material: stainless } \\
& \text { steel } \\
\text { - } & \text { shield gas: Ar }\end{array}$ & VAFS & $\begin{array}{ll}\text { - } & \text { precursor heated to } 373 \mathrm{~K} \\
\text { mixed-shaped particles in the } \\
\text { size range of 3-6 nm with } \\
\text { extremely narrow size } \\
\text { distribution } \\
\text { - } \\
\text { - } \\
\text { no significant aggregation } \\
\text { only anatase phase was } \\
\text { present }\end{array}$ \\
\hline [194] & $\mathrm{CH}_{4} / \mathrm{O}_{2}$ & $\begin{array}{l}\text { TTIP } \\
\text { (Ar as carrier gas) }\end{array}$ & $\begin{array}{ll}\text { - } & \text { premixed, electric field } \\
& \text { assisted flame } \\
\text { - } & \text { burner material: quartz } \\
\text { - } & \text { shield and sheath gas: } \mathrm{N}_{2}\end{array}$ & VAFS & $\begin{array}{ll}\text { - } & \text { precursor heated to } 398 \mathrm{~K} \\
\text { - } & \text { almost exclusively spherical } \\
\text { particles }\end{array}$ \\
\hline [195] & $\mathrm{H}_{2} / \mathrm{O}_{2}$ & $\begin{array}{l}\text { titanium ethoxide } \\
\text { (He as carrier gas) }\end{array}$ & $\begin{array}{ll}\text { - } & \text { low pressure flat-flame } \\
\text { - } & \text { burner material: copper }\end{array}$ & VAFS & $\begin{array}{l}\text { mostly polyhedral particles } \\
\text { with narrow size distribution }\end{array}$ \\
\hline$[196]$ & $\mathrm{CH}_{4} / \mathrm{O}_{2}$ & $\begin{array}{l}\text { TTIP } \\
\text { (Ar as carrier gas) }\end{array}$ & $\begin{array}{ll}\text { - } & \text { premixed flame } \\
\text { - } & \text { burner material: stainless } \\
& \text { steel } \\
\text { - } & \text { shield and sheath gas: } \mathrm{N}_{2}\end{array}$ & VAFS & $\begin{array}{ll} & \text { precursor heated to } 398 \mathrm{~K} \\
\text { - } & \text { almost exclusively spherical } \\
& \text { particles }\end{array}$ \\
\hline [197] & $\mathrm{CH}_{4} / \mathrm{O}_{2}$ & $\begin{array}{l}\text { TTIP } \\
\text { (air as carrier gas) }\end{array}$ & $\begin{array}{ll}- & \text { diffusion flame } \\
- & \text { burner material: } \mathrm{n} / \mathrm{a}\end{array}$ & VAFS & 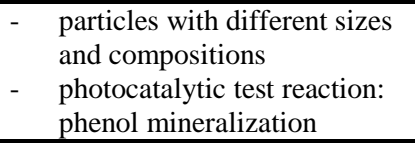 \\
\hline [198] & $\mathrm{CH}_{4} / \mathrm{O}_{2}$ & $\begin{array}{l}\mathrm{TiCl}_{4} \\
\text { (Ar as carrier gas) }\end{array}$ & $\begin{array}{ll}- & \text { electric field assisted, } \\
\text { premixed laminar flame } \\
\text { - } \\
\text { burner material: } \\
\text { aluminium }\end{array}$ & VAFS & $\begin{array}{l}\text { particle shape and phase } \\
\text { composition are questionable } \\
\text { in lack of adequate } \\
\text { measurements }\end{array}$ \\
\hline
\end{tabular}

Table 1: Some details of the most fundamental works focusing on AFS-based $\mathrm{TiO}_{2}$ synthesis.

From AFS techniques we applied the vapor-fed type being discussed in details later on. Here, volatile precursors are needed in order to ensure high enough gas phase concentration, i.e., productivity. The most appropriate component for this purpose is $\mathrm{TiCl}_{4}$ [199] since it has high equilibrium vapor tension under ambient circumstances. Ti-alcoholates (TTIP: titanium tetraisopropoxide) with short carbon 
chains are also suitable materials $[188,191]$. Although they are less volatile than $\mathrm{TiCl}_{4}$, their handling is more convenient due to the lower hydrolyzation rate. Premature hydrolyzation, which may cause serious operational problems (choking of tubes) or uncontrolled crystal growth, could be prevented using water-free gases for precursor vapor transfer as it was done in all works listed in Table 1.

Basically, there are three major process parameters in flame synthesis influencing particle dynamics and, consequently, product characteristics: flame temperature, precursor concentration and residence time [179]. These are determined through directly adjustable operational variables, such as gas flow rates, fuel/oxidant ratio, chemical nature of the precursor and of the gases. Moreover, burner geometry and flame configuration also have a crucial role, as it will be explained in the following paragraphs.

Theoretically, combustion of a given fuel-oxygen mixture releases the highest thermal power at its stoichiometric ratio. Altering this composition in either way will inevitably result in a temperature drop. However, increasing the fuel concentration may have another effect that is to be considered: when oxidant level is lower than that of the stoichiometric amount, the excess fuel will not entirely be burnt causing unwanted carbonaceous contaminations in the reaction zone, if a hydrocarbon is used as fuel. This can lead to doping or to the formation of carbon deposits on the particle surface [200]. Certainly, partaking of external (ambient) oxygen in the oxidation should be taken into account if its influx is not obstructed to a higher extent. When the fuel/oxidant ratio is changed through increasing the flow rate of any of the gases, temperature decrease will be further enhanced by the concomitant higher heat dissipation.

Gas velocities determine not only the flame temperature but also the precursor concentration inside the flame. The extent of dilution substantially affects the rate of basic chemical and physicochemical processes occurring during particle formation, which phenomena will be treated more in the next section. Apart from that, precursor concentration may have drastic contribution to the flame temperature, especially when Ti-alcoholates are used. For instance, the combustion enthalpy of TTIP is measured to be as high as $\Delta \mathrm{H}_{\text {Combustion }}=-7547 \mathrm{~kJ} / \mathrm{mol}$ [201], so a relatively small concentration increase eventuates in significant changes of the product properties. Conversely, $\mathrm{TiCl}_{4}$ produces much less heat $(-140 \mathrm{~kJ} / \mathrm{mol})$, therefore less effect is expected on synthesis conditions (at least concerning flame temperature). There is another possibility of controlling precursor concentration besides regulating the gas flow rates used either for generating the flame or carrying the precursor. In case of VAFS techniques, where the precursor vapor and not its aerosol is introduced into the reactor (as in FSP mode), the gas phase concentration can be easily increased via heating the precursor container. This solution is beneficial over flow rate adjustment, because it does not vary any other synthesis parameter. This statement applies only to cases when the combusted precursor amount stays low enough not to considerably modify the enthalpy content of the flame. $\mathrm{For}_{\mathrm{TiCl}}$, it 
means a significantly broader concentration range than that of TTIP. The criterion of complete combustion has to be fulfilled also for precursors (especially for carbon-containing ones), otherwise formation of metastable aggregates and incomplete crystallization likely occurs [189] which may be detrimental to photocatalytic activity.

Another important parameter in flame methods is the residence time of precursor molecules and particles. The most relevant factor here is obviously the flow rates of gases, nevertheless, the flame configuration and size have influence on it as well [202-203]. This can be justified by considering that in a shorter flame, particles spend shorter time in the hottest luminous zone compared to a longer flame, if same gas velocities are assumed. Such a situation may be achieved by modifying the fuel/oxidant ratio or the precursor concentration. Different flow types (laminar or turbulent), flame types (premixed or diffusion) and burner configurations also differ regarding the observable flame temperature profile $[179,202]$. In connection with this, the method and location of product collection have equally high importance in flame synthesis than the previous operational factors. Here, the quenching (cooling) rate is the most crucial since below a certain temperature, chemical and physicochemical processes driving particle formation cannot gain enough energy to remain activated, so the actual crystallinity, particle and aggregate sizes become frozen below that temperature limit [179].

In summary, one can draw the conclusion from the above deduction that the time-temperature history of the particles is a complex parameter which globally determines product characteristics. In the next chapter, we will give a short description of principles and phenomena governing particle formation in flames and of their relation to basic process parameters.

\subsection{Gas phase particle formation and particle dynamics in flames}

Gas-to-particle conversion has the advantage of allowing particles to be built from molecules all the way up to the desired size. That means better adjustability of product grain sizes compared to flame spray pyrolysis method, where improperly controlled droplet size can further widen size distribution.

Precursor gases flow and react at high temperatures to form product molecules. Typically, these molecules result quickly in particles either by uninhibited molecular collisions (coagulation-driven process) or by nucleation of subcritical molecular clusters (nucleation-driven process), depending on thermodynamics of the given reaction system [204]. In case of unstable clusters, particles are formed by homogeneous nucleation: balanced condensation and evaporation of molecules to and from clusters of product species, respectively. For $\mathrm{TiO}_{2}$, a critical radius of $3 \AA$ was reported by Ulrich 
[205], above which particles further grow by condensation (onto the surface) and/or coagulation. Coagulation - a process by which particles collide and get connected by Van der Waals forces leading to agglomerates - is dominant at the very early stages where particles are extremely small and present in high density. Passing further along the flame, coagulated particles tend to sinter and coalesce due to the high temperature producing aggregates and bigger particles. There is a profound difference between the two kinds of associations described. While agglomerates can be broken up transmitting a relatively small amount of energy to the system (e.g., sonication), aggregates (often termed as "hard agglomerates") containing covalently bonded particles cannot be disrupted by conventional means. The presence of the latter is detrimental to most applications: besides reducing specific surface area, it eliminates the benefits of narrow size distribution [195]. Incidentally, aggregates are well recognizable in TEM (transmission electron microscopy) images from sintering necks appearing between particles. It was pointed out, that for many flame-made oxides (including titania), coagulation normally prevails over surface growth [205,206]. Nanoparticle systems displaying Brownian coagulation - the expression refers to a type of thermophoretic motion performed generally by particles in flames - show a so-called self-preserving size distribution [207], which sets a lower limit on the degree of dispersity. Narrower size distribution, however, is achieved in those cases, when surface growth rather than coagulation is determinant during particle formation [208].

Aggregates as well as bigger particles originate essentially from coagulation and subsequent sintering [204]. The relation of the rates of these two processes is decisive with respect to product quality. If collision rate is higher than that of sintering, aggregated particles are formed. On the other hand, if sintering is faster than particle collision, then regular-shaped, monolithic particles are obtained [209]. The collision rate between particles is dictated by the precursor concentration and the flow type, while sintering rate depends on the material, primary particle size and temperature. It is easily predictable that higher residence time and temperature will favor increased sintering rate, and higher precursor concentration yields enhanced collision rate. Therefore, increasing any of these three parameters (if the flow and the precursor type are assumed unchanged, as normally the case is) eventually leads to bigger aggregates and/or bigger primary particles [188]. It was previously shown, that surface reactions have higher significance at the early stages, then growth continues with simultaneous coagulation and sintering/coalescence. Leaving the luminous flame behind, primary particle growth stops as the temperature drops down to an extent not facilitating sintering further. From this point, particle collision results in agglomerates [196].

For achieving narrow size distribution and uniform catalyst quality, particles must experience nearly the same time-temperature history along their trajectory. Premixed flames are reported to support this demand the most, since the inner flame volume does not contain steep temperature gradients due to the efficient fuel-oxidant mixing [210]. In co-flow diffusion mode, reactant mixing 
is far from perfect. Here, a less homogeneous temperature distribution exists both in axial and radial direction, as burning takes place at well-determined flame fronts where components first get in touch [203]. This situation can, however, be improved to some extent, when the flow has at least partially turbulent character (in a controlled fashion) providing better mixing conditions. Naturally, a drastic temperature drop is observed for both flame types heading outwards in radial direction from the flame center when passing through the luminous flame boundary. Another requirement of product uniformity is stable flame operation. Disturbances causing temporal variation of the temperature profile will obviously widen the size distribution. Nevertheless, stability is ensured in most works treated by the current overview with the aid of appropriate burner design.

It is also important to inspect the phase evolution of $\mathrm{TiO}_{2}$ particles during particle formation. Some authors postulated [193,211] that initially, anatase clusters are present which either grow to anatase particles or transform into rutile clusters when reaching the thermodynamically critical size. Morooka et al. [211] assumed that, though this transformation rate is rapid, the process suddenly stops as soon as anatase particles are formed from clusters of the same phase. It is important to mention here, that this theory rules out the existence of chemically bound phases claimed to be responsible for an enhanced charge separation [160-161]. Anyhow, flame synthesis methods provide $\mathrm{TiO}_{2}$ mostly in a physical mixture of anatase (majority) and rutile ([212-213] and those referred in Table 1), just as we experienced in our work.

There are several interesting improvements of the flame synthesis method implemented to avoid higher magnitude of aggregation. A few of them applies low pressure flames [195], where interparticle collisions are successfully minimized. This stems from the observed low flame temperature, short residence time and higher flame radical concentration allowing the production of non-aggregated, perfectly crystalline $\mathrm{TiO}_{2}$ particles. Another technique suitable for achieving this goal is the electric field enhanced flame synthesis, in which the flame is located between two plate electrodes [194,198]. Field strength adjustment enables flame height regulation (i.e., residence time), moreover the mutual repulsion of unipolarly charged particles retards collision rate, further increasing the efficiency in producing non-aggregated nanoparticles.

In our case, the lack of suitable instrumentation (chamber with pressure control, IR-monitoring, etc.) and the intention of developing a less complicated and easily scalable flame method led to the flame reactor design presented herein. Despite its simplicity, it was able to reproducibly synthesize catalyst samples overtaking the photoactivity of P25 in the breakdown of several model compounds. The only drawback is its poor productivity basically ascribed to VAFS methods, because they are using the precursor in vapor form. However, increasing the number of burners and/or gas volume velocities, or re-designing the evaporator in order to provide bigger liquid-air interfacial area could readily solve this issue. In addition, our primary aim was not to produce big quantities but to 
understand the underlying principles of the synthesis itself and of parameters-structural properties interrelations, which collectively provided a basis for further process optimization towards reaching the highest possible activities.

\section{Objectives}

In this work, a VAFS method was utilized to synthesize nanostructured $\mathrm{TiO}_{2}$ photocatalysts. This procedure is of particular importance, since it has numerous benefits over other conventional technologies. Moreover, one of the most efficient photocatalysts - marketed as P25 - is manufactured in this way. Our primary aim was to produce $\mathrm{TiO}_{2}$ samples with the highest achievable photocatalytic activity via synthesis optimization. We have thoroughly investigated the chemical and structural characteristics of the samples by different analytical methods. We attempted finding correlation between activity and sample properties. For this, photocatalytic tests were carried out both in gas phase and in aqueous media, using three different model compounds. Comparison of these results provided valuable information on the medium and substrate dependency of catalytic performance. With respect to environmental applications, where usually utter decomposition of harmful pollutants is required, it is necessary to have a complete picture about the overall mineralization efficiency of the given photocatalyst. Accordingly, total organic carbon (TOC) removal rates, oxygen consumption rates and intermediate concentration profiles were also analyzed for our flame-made samples.

\section{Experimental section}

\subsection{Preparation of $\mathrm{TiO}_{2}$ samples by vapor-fed aerosol flame synthesis (VAFS)}

Despite its existing but not too severe limitations compared to other flame-based techniques, we decided to use a co-flow diffusion burner for different reasons. On the one hand, separation of flame-generating components was believed to carry lower risk of an accidental flashback. Secondly, it is well-known that, in industry, diffusion flame reactors (albeit in turbulent mode) are often applied for manufacturing different powders (like $\mathrm{TiO}_{2}$ ) at a rate of tons per hour [179]. Apart from this high productivity, $\mathrm{TiO}_{2} \mathrm{~S}$ made in laboratory-scale diffusion flames showed considerable activity in the decomposition of different hydrocarbons [197,213]. 

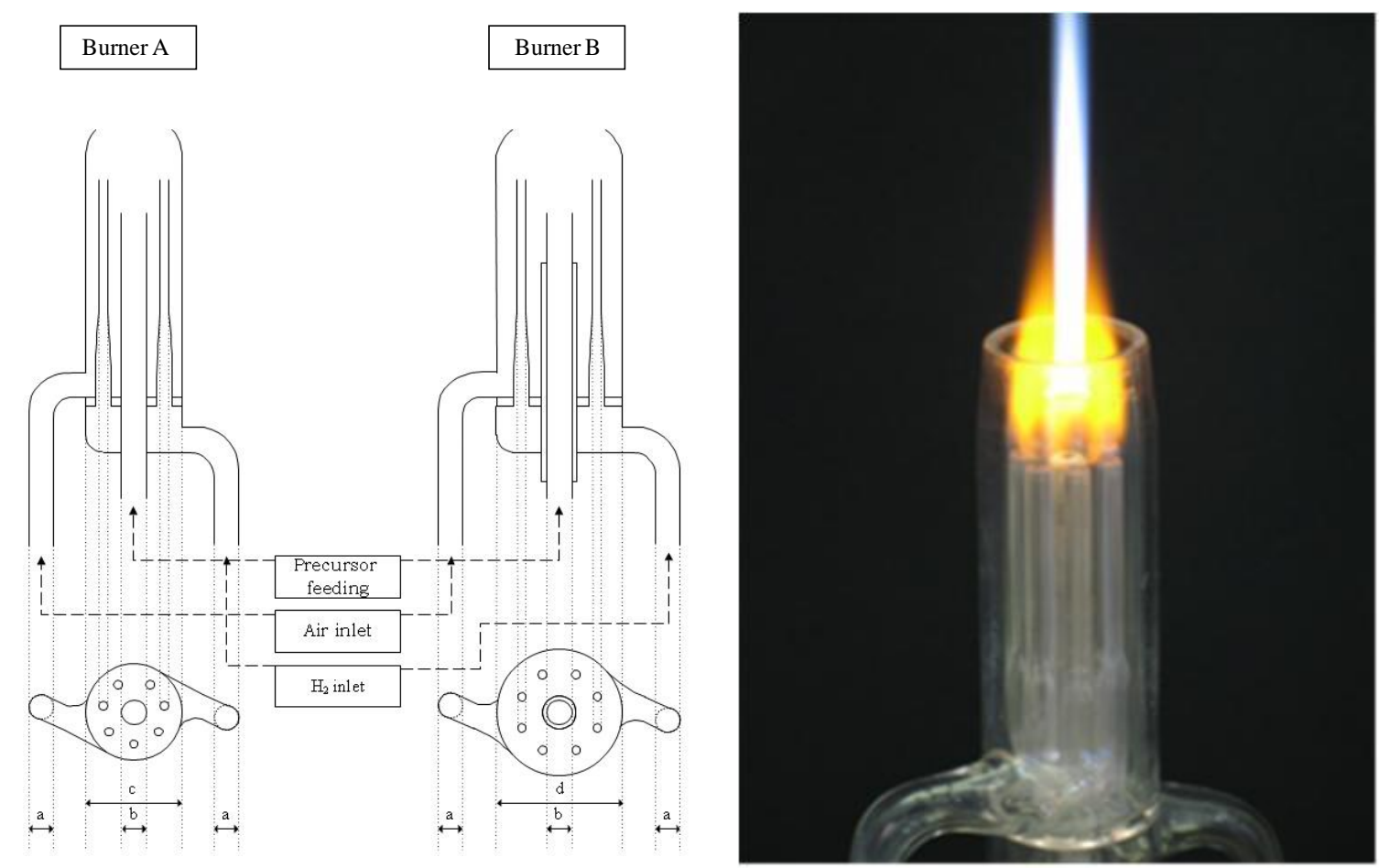

Figure 3: Schematic cross-sectional top- and side-views of our burners (left image) and Burner-A during operation (right image). Sizes indicated with lower case letters in the left image are the following: $a=5 \mathrm{~mm} ; \mathrm{b}=3 \mathrm{~mm} ; \mathrm{c}=27 \mathrm{~mm} ; \mathrm{d}=35 \mathrm{~mm}$

Two home-made burners (modified versions of a commercially available one), with slightly different geometry were used for producing photocatalyst samples. These co-flow diffusion flame reactors (Burner-A and B; Figure 3) were equipped with a bubble vapor generator (Figure 4, item 1) containing the precursor $\left(\mathrm{TiCl}_{4}\right)$. Both reactors consisted of a central tube through which $\mathrm{TiCl}_{4}$ was fed into the flame. The fuel $\left(\mathrm{H}_{2}\right)$ intake was made by seven (Burner-A) or eight capillaries (BurnerB) situated concentrically around the precursor vapor feeding tubes. This way the air acted both as oxidant and as sheath gas (similarly to the design described in refs. [188,214,215,216,217]). BurnerA was made of glass, while the upper $\sim 5.5 \mathrm{~cm}$ part of Burner-B was constructed from quartz - glued to the lower part by a special adhesive - in order to provide better heat resistance. The inner diameter of capillary tubes $(1 \mathrm{~mm})$, the hydrogen and air intake tubes $(\mathrm{a}=5 \mathrm{~mm})$ and of the vapor feeding tubes $(b=3 \mathrm{~mm})$ were the same for both burners, but the overall volume of their bodies differed $(\mathrm{c}=$ $27 \mathrm{~mm}, \mathrm{~d}=35 \mathrm{~mm}$ in Figure 3). Consequently, the distance between the capillary tubes is larger for Burner-B than for Burner-A. There was a removable auxiliary glass tube fitted onto the inner precursor feeding tube in Burner-A (not indicated on the schematic). This upper piece was exposed to heat the most, therefore, its orifice got fused and shrunk always to the same diameter in a couple of minutes after igniting the flame. Prior to each synthesis run, this small upper tube could be replaced by a clean and undistorted new one, rendering the procedure more reliable and reproducible. By contrast, Burner-B was ab ovo made with a displaceable precursor feeding tube, whose vertical position was set slightly below the high temperature zone. The practicability of such unrestricted 
positioning necessitated the design of a larger burner, leading eventually to the alteration of the general operational conditions.

The experimentally accessible parameter ranges of both burners have been determined in preliminary runs, where formation of stable flames with optimal geometry was the main consideration. The outcome of these optimization steps is summarized hereafter. For Burner-A, the total air flow rate was changed between 4841 and $7707 \mathrm{~mL} / \mathrm{min}$ and the hydrogen flow was kept constant $(1230 \mathrm{~mL} / \mathrm{min})$ in order to set the desired hydrogen-oxygen molar ratio in the flame (0.76 to 1.21). The flow rate of air bubbling through the $\mathrm{TiCl}_{4}$ was systematically changed from 118 to 355 $\mathrm{mL} / \mathrm{min}$. This portion was split off from the above-specified total air stream by a Teflon-inlayed glass valve. Volumetric flow rates of all other branches were regulated and monitored by calibrated floating ball rotameters (Cole-Parmer). Since Burner-B's dimensions are somewhat different from those of Burner-A, optimal flame geometry is obtained with other parameter sets. The total air stream was varied between 6422 and $7098 \mathrm{~mL} / \mathrm{min}$ and the hydrogen flow was set to $1422-1810 \mathrm{~mL} / \mathrm{min}$, thus keeping the overall gas flow rate roughly identical $(8193-8675 \mathrm{~mL} / \mathrm{min})$ at each run. The hydrogenoxygen molar ratio was controlled to fall within 1.00-1.41 and the $\mathrm{TiCl}_{4}$ flow was adjusted between 178 and $710 \mathrm{~mL} / \mathrm{min}$. In the case of Burner-B, we also tested the effect of the liquid precursor temperature, it was increased from the normally used $30^{\circ} \mathrm{C}$ to 50 and $70^{\circ} \mathrm{C}$. Moreover, unlike at Burner-A, we have had the opportunity to conduct syntheses with $20 \%$ lower and higher overall flow rates. At both latter series, all changes introduced were derived from the parameter set belonging to the most efficient sample obtained until that given point of time.

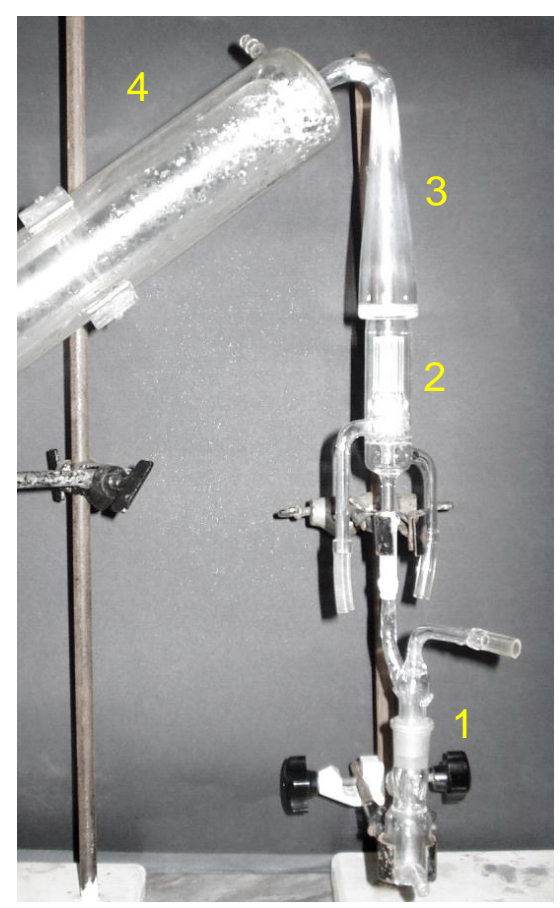

Figure 4: The complete VAFS experimental setup applied in our study. Annotations: 1: $\mathrm{TiCl}_{4}$ container; 2 : Burner-A; 3: quartz chimney; 4: $\mathrm{TiO}_{2}$ collector 
The $\mathrm{TiO}_{2}$ aerosol produced in the flame was driven to a water-cooled glass collector (Figure 4, item 4) through a conical quartz chimney (Figure 4, item 3). Particles collected in this trap are definitely not subjected to higher temperatures any longer (over a few hundred or even a thousand of ${ }^{\circ} \mathrm{C}$ ) in contrast with those methods, where powders are gathered on filters continuously exposed to the flame [218]. Namely, our $\mathrm{TiO}_{2}$ particles go through a rapid quenching when smashed into the condensed water film formed on the inner surface of the trap. On the other hand, samples collected on dry filters can experience extreme temperatures for longer time intervals, this way showing higher rate of aggregation and sintering. In our synthesis procedure, not every grain was trapped in the liquid phase as evidenced by productivity calculation and visual observation (outgoing white smoke of $\mathrm{TiO}_{2}$ at the condenser orifice). These escaping particles - having a very different quenching rate compared to those collected - did not contribute to size distribution broadening. Accordingly, sintering necks between particles appeared only a few times on TEM images (Figure 9) proving a lower extent of longer lasting high temperature exposure-induced "secondary" aggregation that may occur after the evolution of primary particles.

Trapped $\mathrm{TiO}_{2}$ samples formed strongly acidic suspensions due to the significant amount of $\mathrm{HCl}$ liberated from the precursor. Their purification was carried out via dialysis against distilled water until conductivities dropped below $20 \mu \mathrm{S} / \mathrm{cm}$. The clean dispersions were dried at $120^{\circ} \mathrm{C}$ and the resulting solid remnants were finally ground to powders in an agate mortar. For ease of better identification, sample naming contains the type of the burner, the hydrogen-oxygen molar ratio, the precursor vapor feed rate in $\mathrm{mL} / \mathrm{min}$ and the temperature (in centigrade) of the liquid precursor. These parameters are separated by hyphens, for example: A-0.96-148-30. $\mathrm{TiO}_{2} \mathrm{~S}$ originating from independent synthesis runs carried out under the same experimental conditions (i.e., reproduced ones) are distinguished by serial numbers added to the previous code after a slash, such as A-0.96-355-30/2. Another naming extension was applied only in two cases of B-sample synthesis, when the total gas flow rate specific to sample B-1.24-355-30 was decreased by $20 \%$ or increased by $20 \%$. These gave sample codes B-1.24-355-30-0.8 and B-1.24-355-30-1.2, respectively.

\subsection{Determination of photocatalytic activities}

\subsubsection{Experiments in gas phase}

Gas phase tests were performed using methanol and acetaldehyde as model compounds. Here, $0.100 \mathrm{~mL}$ of stock $\mathrm{TiO}_{2}$ suspensions $(300 \mathrm{mg} / \mathrm{L})$ was evenly distributed on surfaces of individual small glass plates (surface area: $3.24 \mathrm{~cm}^{2}$ ) to obtain $\mathrm{TiO}_{2}$ films with uniform thickness. Every plate 
was then dried in a drying cupboard at $100^{\circ} \mathrm{C}$ for 60 minutes and placed on the bottom of a gastight reaction vessel (volume: $20 \mathrm{~mL}$ ). The as-prepared samples were irradiated for 30 minutes prior to the photocatalytic test for removing adventitious organic contaminations. Subsequently, a calculated amount of the saturated vapor of the given model compound was injected into the closed reactor. The initial concentration of the substrates was $1.2-1.4 \times 10^{-4} \mathrm{M}$ and no measurable adsorption was observed for any of them. Reactors were irradiated by a fluorescent lamp with a radiation maximum at $365 \mathrm{~nm}$. The intensity of UV light was $\mathrm{I}=7.23 \pm 0.06 \times 10^{-6}$ einstein $\mathrm{s}^{-1}$ measured by utilizing ferrioxalate actinometry [72]. The gas phase concentration of the substrates was determined by a GC system consisting of an HP 5890 gas chromatograph and a Supelcovax 10 capillary column $(30 \mathrm{~m} \times 0.53$ $\mathrm{mm}$, inner diameter: $0.50 \mu \mathrm{m})$. Samples, taken out from the reaction vessel by a chromatography syringe, were analyzed using nitrogen eluent and flame ionization detector.

\subsubsection{Experiments in liquid phase}

Photocatalytic performances of all catalysts were characterized by aqueous phenol decomposition. The photoreactor $(\mathrm{V}=100 \mathrm{~mL})$ was an open quartz tube with double walls, surrounded by a thermostating jacket $\left(25.0 \pm 0.1^{\circ} \mathrm{C}\right)$. As light sources, six external fluorescent lamps were placed symmetrically around the continuously stirred reactor (Figure 5). Their main parameters were the following: $6 \mathrm{~W}$ power, emission maximum at $365 \mathrm{~nm}$, UV-intensity $\mathrm{I}=1.02 \pm 0.01 \times 10^{-6}$ einstein $\mathrm{s}^{-1}$ (based on ferrioxalate actinometry) [72]. The concentration of phenol was determined with the aid of an HPLC system consisting of a Merck-Hitachi L-7100 low-pressure gradient pump equipped with a Merck-Hitachi L-4250 UV-vis detector and a Lichrospher RP18 column. Methanol/water mixture in a ratio of 5/9 served as eluent. Detection wavelength was set to the lower absorption maximum of phenol $(\lambda=210 \mathrm{~nm})$. Appearance of primary degradation products (PC and HQ) were also monitored during analyses. The initial concentration of the substrate was $5.0 \times 10^{-4} \mathrm{M}$ and a $1 \mathrm{~g} / \mathrm{L}$ photocatalyst concentration was used throughout. No adsorption of phenol was observed (even at $4 \mathrm{~g} / \mathrm{L}$ photocatalyst loading), therefore the actual concentration of the substrate in the solution phase was considered to be equal to the concentration of unreacted phenol. Samples were taken from the suspension in predetermined time intervals, for 120 minutes. Besides phenol, no other additives ( $\mathrm{pH}$ - and ionic strength-affecting compounds, etc.) were used. Since the presence of dissolved oxygen is substantial for the mineralization of phenol [61,219], we applied continuous air bubbling during the photocatalytic tests. Before starting the illumination, the suspension was subjected to 5 minutes of sonication and was held in the reactor in dark for 10 minutes ensuring thermal and mass transport equilibria to be set. 


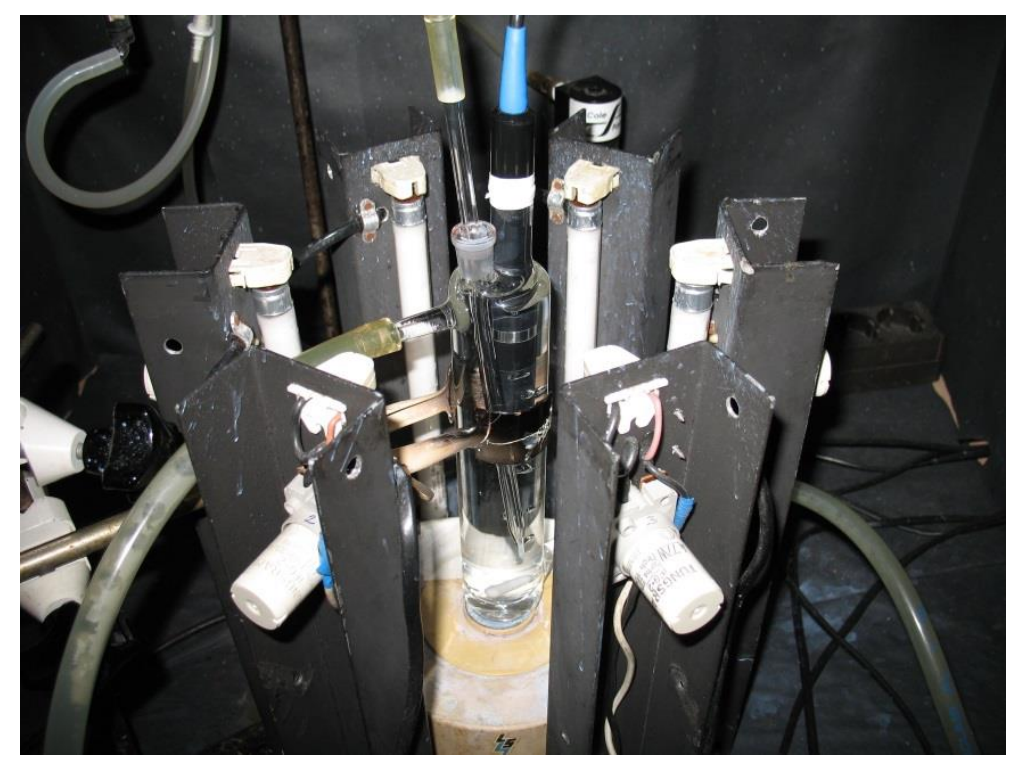

Figure 5: Experimental setup of aqueous phenol decomposition. Six fluorescent lamps are surrounding the photoreactor in which the glass air-inlet tube and an oxygen-sensitive biamperometric cell (black plastic tube) are immersed.

At both gas and liquid phase evaluations, the initial degradation rate of model compounds, $\mathrm{r}_{0}$, was considered the measure of the photocatalytic efficiency. To determine $r_{0}$, a second order polynomial function was fitted to the experimentally observed $\mathrm{c}=\mathrm{f}(\mathrm{t})$ data points up to different conversion ranges; for phenol $<60 \%, 60 \%$ for acetaldehyde and $100 \%$ for methanol. The fitting provided satisfactory description of the data set as regression coefficients $\left(\mathrm{R}^{2}\right)$ were always above 0.98 (but in most cases it fell even closer to 1). Finally, the slope of this empirical function at $\mathrm{t}=0$ yielded the initial rate of the photocatalytic reaction (Figure 6).

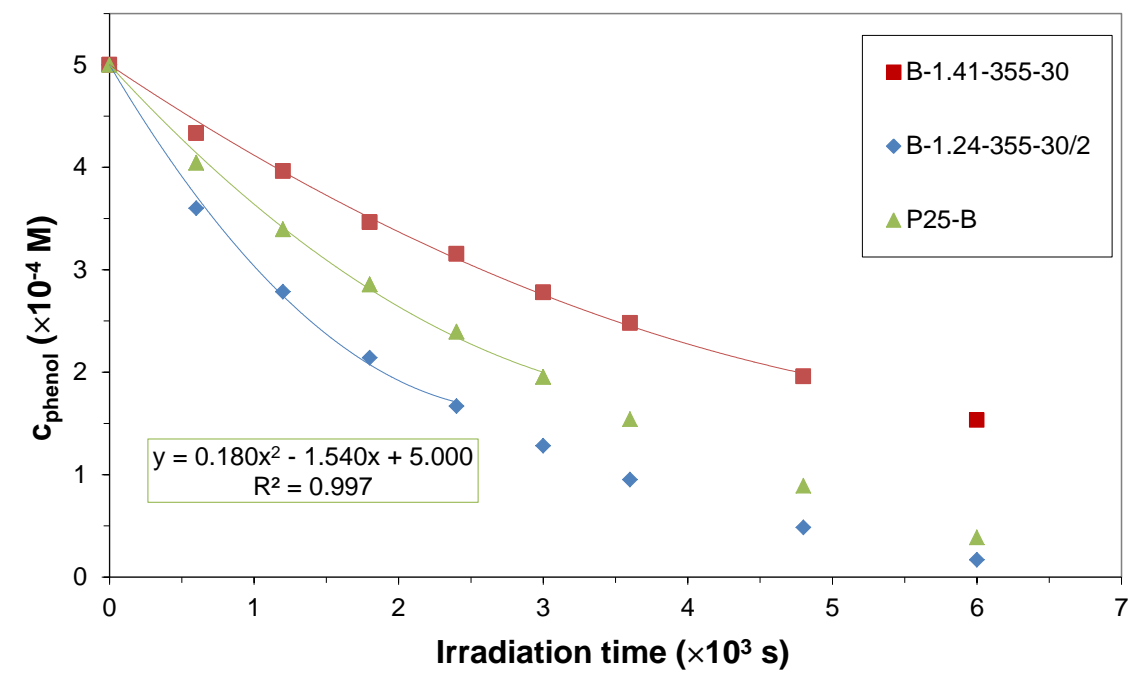

Figure 6: Examples for phenol decomposition curves: the actual phenol equilibrium concentration is plotted against the time of UV-irradiation. In green frame, trendline parameters of P25-B's second order polynomial fitting are given. 
It is to be mentioned that - unless otherwise stated - liquid phase photocatalytic activities will exclusively be used in all further evaluation steps and representations instead of gas phase ones. Gas phase measurements have been introduced later to check whether the individual catalytic performances are comparable when applying a different medium and different model compounds. By contrast, phenol decomposition was exhaustively studied and routinely used throughout many years in our research group and, therefore, its technical implementation reached a very sophisticated level. Here, unlike at gas phase investigations, the experimental setup, the reaction parameters and the analytical methodology were all fully optimized to the given purpose.

\subsection{Characterization methods}

\subsubsection{X-ray diffractometry $(X R D)$}

$\mathrm{X}$-ray diffractometry (XRD) was employed in order to characterize the average particle size and phase composition of catalysts. Two different instruments were used: a Philips PW 1820/1830 diffractometer for samples made by Burner-A and a Rigaku Miniflex II benchtop XRD system for sample series B. All measurement conditions were the same in both cases $(\mathrm{Cu} \mathrm{K \alpha}=0.15406 \mathrm{~nm}, 40$ $\mathrm{kV}$, and $30 \mathrm{~mA}$, in the $20 \leq 2 \Theta^{\circ} \leq 40$ regime). The average particle diameters (d) were obtained by means of the Scherrer equation [220]:

$$
d=\frac{k \alpha}{\beta \cos \theta}
$$

where $\beta$ is the line broadening ( $\beta=\beta_{\mathrm{S}}-\beta_{0}$, where $\beta_{\mathrm{S}}$ and $\beta_{0}$ are XRD peak half-widths of the sample and of the silicon standard), $\mathrm{k}$ is related to the crystallite shape $(\mathrm{k}=0.9$, when assuming sphere-like particles), $\alpha$ and $\Theta$ are the radiation wavelength and Bragg angle, respectively. A single crystal silicon standard $\left(\beta_{0}=0.1058\right)$ was used for calibration. To ascertain the phase composition of catalyst powders, primary diffraction peaks of anatase $(101)\left(2 \Theta=25.3^{\circ}\right)$ and rutile $(110)\left(2 \Theta=27.5^{\circ}\right)$ were integrated. It turned out from the results that samples contained anatase and rutile phase only. The presence of amorphous phase was ruled out by a calibration method, in which a series of nanocrystalline anatase-rutile mixtures covering the whole composition range was measured. The weight fraction of rutile, $\mathrm{W}_{\mathrm{R}}$, can be calculated directly from peak area values by the following equation [155]: 


$$
W_{\mathrm{R}}=\frac{A_{\mathrm{R}}}{0.884 A_{\mathrm{A}}+A_{\mathrm{R}}}
$$

where $A_{R}$ and $A_{A}$ are the rutile (110) and the anatase (101) peak, respectively.

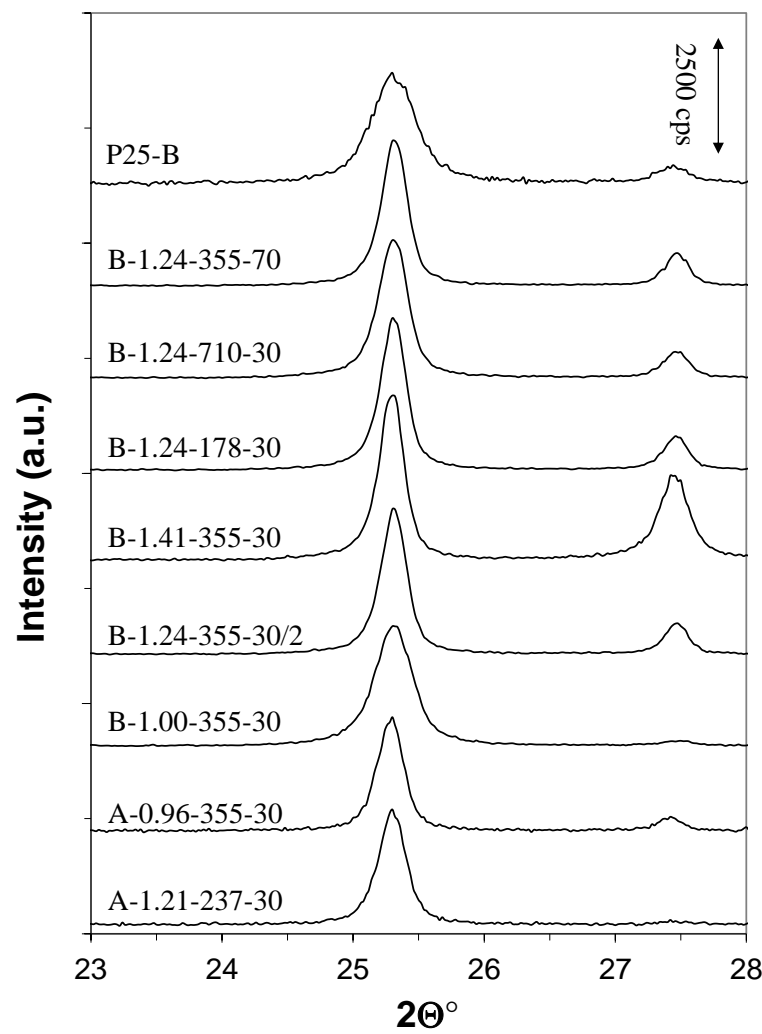

Figure 7: X-ray diffraction patterns of a few samples. Anatase and rutile reflections are seen at $2 \Theta=25.3^{\circ}$ and $27.5^{\circ}$, respectively.

\subsubsection{Transmission electron microscopy (TEM)}

Transmission electron microscopic (TEM) studies were performed to determine particle sizes and size distributions and, also, to observe the morphology of particles. TEM micrographs were recorded on a Phillips CM 10 instrument operating at an accelerating voltage of $100 \mathrm{kV}$. Dilute aqueous suspensions ( $\mathrm{ca} .10 \mathrm{mg} / \mathrm{L}$ ) of the samples were dripped up onto Formvar-film-coated copper grids, which were placed into the microscope only after proper drying. The micrographs were evaluated by the aid of a special image analyzer software (analySIS). In order to ensure representative sampling of our statistics, 800-1000 grains were manually counted and images (each containing more than 50 crystallites) were taken from at least 8-10 different regions of the TEM grids. According to this demand, we usually applied a 92k-fold magnification.

TEM is a unique tool amongst those applied in the present study, that is capable of providing not only the average but also the individual diameter of particles. From this data, the valuable 
information of size distribution can easily be derived. It is well-seen from the above evaluation method description, that for ensuring proper statistical basis, quite a high number of grains had to be measured manually. Therefore, we decided to apply TEM mostly for cornerstone B-samples of each experimental series (i.e., catalysts synthesized with - for instance - the highest and lowest $\mathrm{H}_{2}-\mathrm{O}_{2}$ molar ratio). This approach was a good compromise, as it still revealed the potential size/morphologyrelated trends while keeping the effort investment at an acceptable level. XRD remained the standard technique for ascertaining the average particle diameters with a single advantage over TEM: average sizes of both existing $\mathrm{TiO}_{2}$ phases could be measured, whereas particles having different phases are not distinguishable on electron microscopic projections. Accordingly, TEM data comprises values of both crystalline phases. This introduces a relatively small and constant error in the size determination as the anatase content of the entire set of samples always fell roughly within 80-100\% (except only a few samples). Another potential source of XRD-TEM average grain size dissimilarity is that in case of oblong particles, only the longest diagonal was measured and recorded at TEM.

On the other hand, XRD also suffers from a certain inaccuracy due to the following limitation: particle diameters are deduced from diffraction peak broadening (Equation 18) which is more and more emphasized when approaching very small particle sizes. In the diameter range of our flamemade $\mathrm{TiO}_{2}$ samples $(40-80 \mathrm{~nm})$, the line broadening becomes very small, that is, its measurement will unavoidably be inaccurate. Nevertheless, the comparison between XRD and TEM results typically brought a difference of $c a$. $0-20 \%$ in sizes and it exceeded this value only in a few instances. All in all, XRD - as being a fast and reliable method - has been chosen as the primary source of particle sizes for both titania phases and, evidently, of the phase composition as well. Consequently, all later representations, where particle size is the dependent or the independent variable, exploit XRD data. Due to its higher effort-demand, TEM was used only in such reasonable cases, when the knowledge of size distribution or particle morphology might help in identifying fundamental interdependences.

\subsubsection{Scanning electron microscopy (SEM)}

Scanning electron microscopic (SEM) images of several samples were taken on a Hitachi S4700 device. The instrument was equipped with a field emission cathode as electron source. Prior to the measurements, gold was evaporated onto each sample in order to obtain the appropriate conductivity. In comparison with TEM, SEM did not provide so much new information, although virtual 3D geometries of $\mathrm{TiO}_{2}$ particles could be visualized exclusively by this technique (Figure 8). In addition,

particle sizes were also double-checked here which showed good similarity to diameters extracted from other methods. 


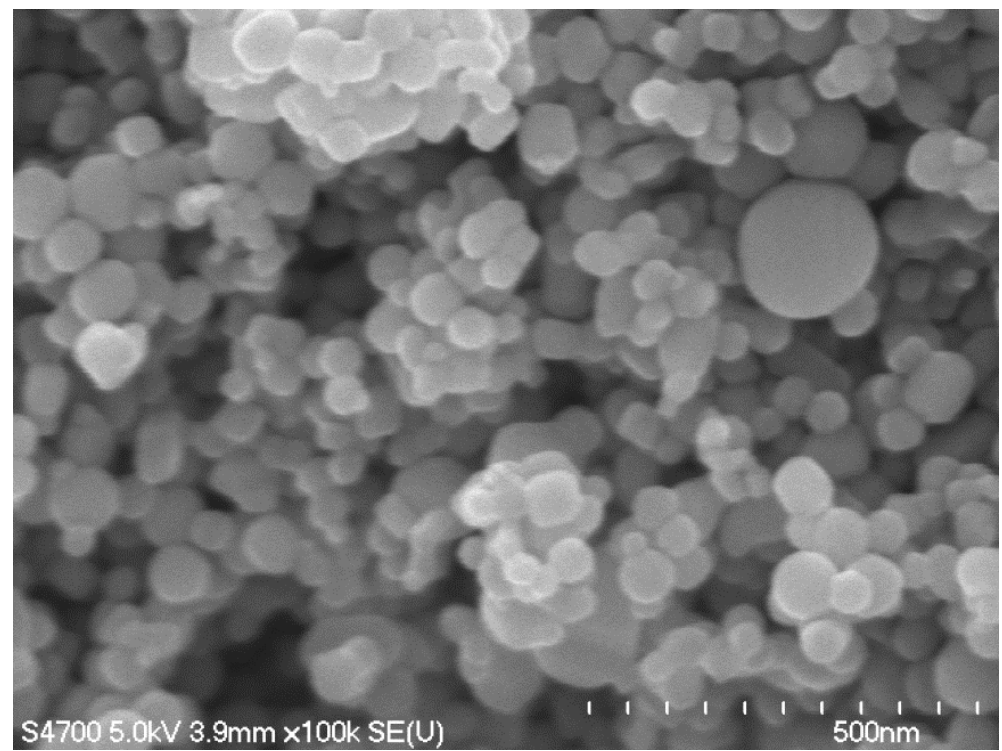

Figure 8: SEM image of sample A-1.21-237-30/2

\subsubsection{High-resolution transmission electron microscopy (HRTEM)}

High-resolution transmission electron microscopic (HRTEM) images were recorded on a JEOL-3010 equipment, operating at $300 \mathrm{kV}$ and having a point resolution of $0.17 \mathrm{~nm}$. The built-in energy dispersive X-ray detector allowed us to check the elemental composition of our samples (elemental map). HRTEM images were used to reveal the crystal structure of individual particles and to look for possible interparticle connections between grains with different crystal phases (Figure 9). Specimens were supported on holey carbon sample holders. 

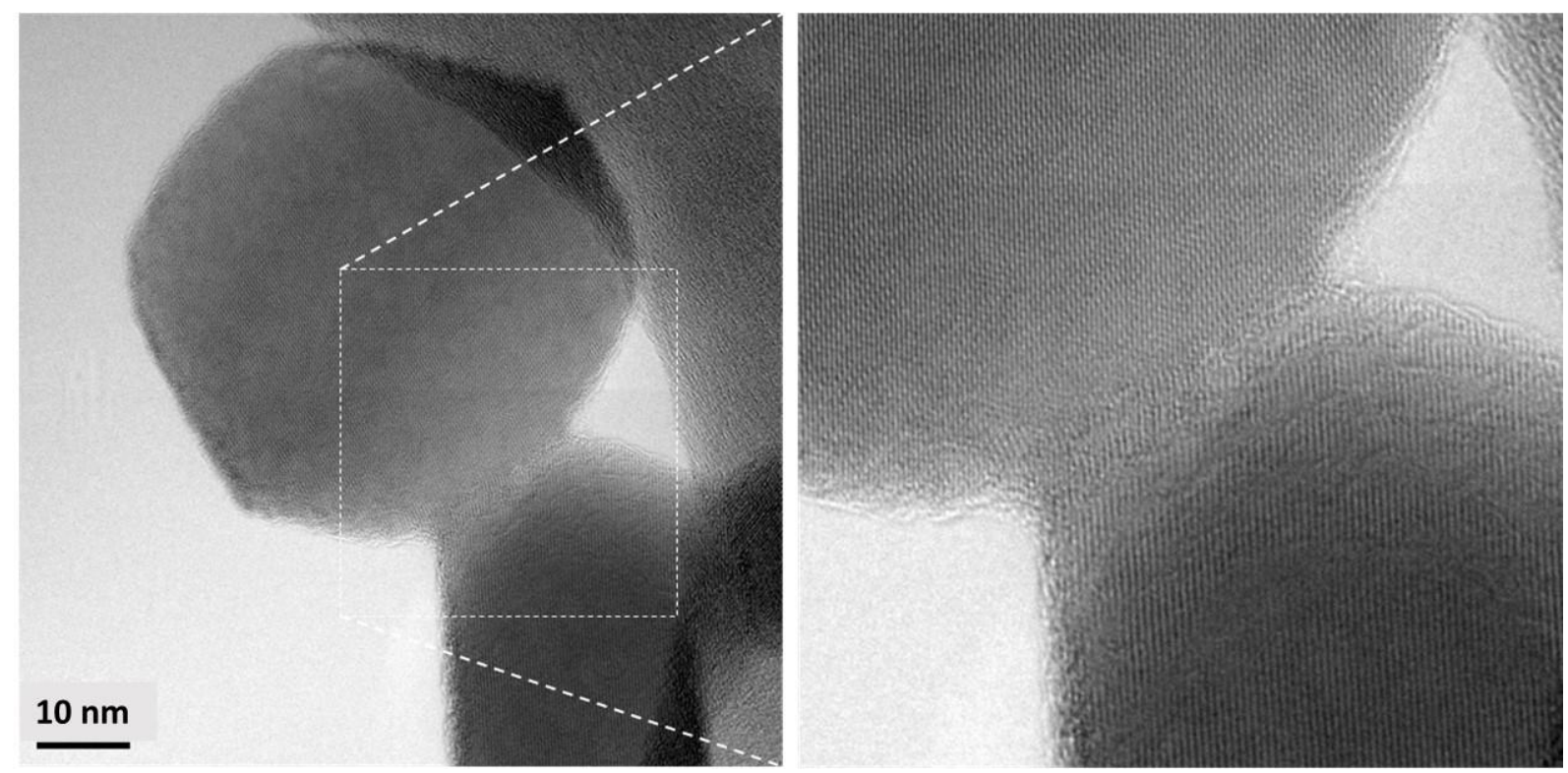

Figure 9: HRTEM image and its magnified version (on the right) on a sintering neck formed between two $\mathrm{TiO}_{2}$ particles. Periodicities of the respective crystal lattices are also visible. The scale bar of the original image represents a length of $10 \mathrm{~nm}$.

\subsubsection{Nitrogen adsorption}

The specific surface area (SSA) of catalysts was determined by nitrogen adsorption at $77 \mathrm{~K}$ by a Micromeritics gas adsorption analyzer (Gemini Type 2375) after vacuum drying of samples at $100^{\circ} \mathrm{C}$ for $12 \mathrm{~h}$. The SSA was calculated using the BET (Brunauer-Emmett-Teller) method. A unique information which can be derived from the shape of the $\mathrm{N}_{2}$-adsorption isotherms is whether particles are porous or not. Since there is no significant hysteresis between the adsorption and desorption curves (Figure 10), our samples are concluded not to be porous.

Taking the entire set of flame-made $\mathrm{TiO}_{2} \mathrm{~s}$, SSAs were found to range between 20 and $45 \mathrm{~m}^{2} \mathrm{~g}^{-1}$. Assuming that samples constitute of uniform spheres, an average particle diameter of $50-80 \mathrm{~nm}$ can be calculated, being in good agreement with those obtained from both XRD and TEM. Accordingly, to avoid "duplicating" data, we decided to reduce the number of measurements at B-sample series and to check sample porosity only at cornerstone-samples further on. 


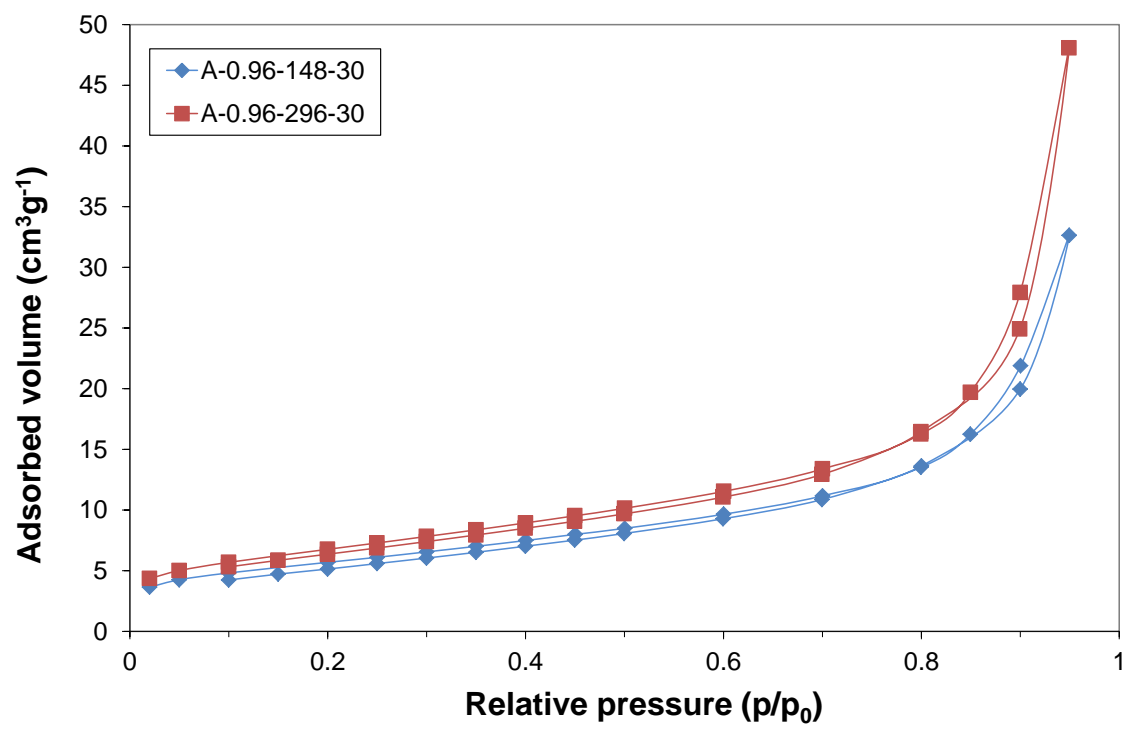

Figure 10: $\mathrm{N}_{2}$-adsorption isotherms for samples A-0.96-148-30 and A-0.96-296-30 with specific surface area of 20 and $24 \mathrm{~m}^{2} \mathrm{~g}^{-1}$, respectively.

\subsubsection{X-ray photoelectron spectroscopy (XPS)}

X-ray photoelectron spectra of selected samples were taken with a SPECS instrument equipped with a PHOIBOS 150 MCD 9 hemispherical electron energy analyzer (FAT mode). The excitation source was the $\mathrm{K} \alpha$ radiation of a magnesium anode $(\mathrm{h} v=1253.6 \mathrm{eV})$. The $\mathrm{X}$-ray gun was operated at $180 \mathrm{~W}(12 \mathrm{kV}, 15 \mathrm{~mA})$. The pass energy was set to $20 \mathrm{eV}$, the step size was $25 \mathrm{meV}$, and the collection time in one channel was $150 \mathrm{~ms}$. Typically, five scans were merged to get a single spectrum. The C 1s binding energy of adventitious carbon served as energy reference $(285.1 \mathrm{eV})$. With this method, the $\mathrm{Ti}^{4+}$ peak of the $\mathrm{Ti} 2 \mathrm{p}_{3 / 2}$ signal was detected at $459.0 \pm 0.2 \mathrm{eV}$. XP spectra provided information on the surface quality through the $\mathrm{Ti}^{4+} / \mathrm{Ti}^{3+}$ and lattice oxygen/OH-group signal ratios. Latter peaks are the main components of the $\mathrm{O} 1 \mathrm{~s}$ signal group located in the binding energy range of $c a$. 525-535 eV. For data acquisition and processing, the software of the equipment manufacturer (SpecsLab2) and a commercial one (CasaXPS, Origin) were used, respectively. Before data analysis, X-ray satellites - originating from the non-monochromatic excitation - were removed.

\subsubsection{Dynamic light scattering (DLS)}

The aggregation behavior and $\mathrm{pH}$-dependent surface charge formation of catalyst particles were studied by dynamic light scattering (DLS) technique, using a NanoZS apparatus (Malvern, UK). The light source was a He-Ne laser $(4 \mathrm{~mW} ; \lambda=633 \mathrm{~nm})$, operating in backscattering mode at the angle of $173^{\circ}$. Measurements were conducted at $25.0 \pm 0.1^{\circ} \mathrm{C}$, in a disposable zeta cell (DTS 1060). $\mathrm{TiO}_{2}$ 
samples were dispersed in $\mathrm{NaCl}$-solutions of $0.01 \mathrm{M}$ and the catalyst content was always set to 10 $\mathrm{mg} / \mathrm{L}$ for ensuring optimal detectable light intensity. The $\mathrm{pH}$ of these suspensions was adjusted in the range of 2-10 by using $\mathrm{NaOH}$ - and $\mathrm{HCl}$-solutions. The as-prepared samples were then sealed and kept undisturbed for a couple of hours (usually overnight) prior to usage in order to achieve equilibrium circumstances. $\mathrm{pH}$ values have been re-checked and recorded before starting the measurements, these data were considered at later evaluation. In a typical experiment, a small portion of dispersion $(\sim 3$ $\mathrm{mL}$ ) was agitated again and ultrasonicated for 10 seconds before placing it into the sample holder. Data acquisition was initialized after a one-minute waiting period. The average zeta potential and hydrodynamic diameter values were calculated from third order cumulative fits of the correlation functions.

\subsubsection{Total organic carbon (TOC) measurements}

Photocatalytic efficiency can equally be expressed as the mineralization rate of a given target compound. The more advanced the process is, the bigger amount of carbon-containing species are transformed into $\mathrm{CO}_{2}$. The complete mineralization of phenol was followed by measuring the total organic carbon (TOC) content of solution samples that have gone through the same photocatalytic tests as described in Section 4.2.2. Measurements were carried out in a Euroglas TOC 1200 apparatus (Delft, The Netherlands). After injecting samples of $100 \mu \mathrm{L}$ into the instrument, they were combusted at $1000^{\circ} \mathrm{C}$ under oxygen-argon atmosphere and the amount of evolving $\mathrm{CO}_{2}$ was finally analyzed by the built-in IR-spectrometer. Standard solutions of oxalic acid were used for calibration.

\subsubsection{Oxygen consumption measurements}

Aqueous phase photocatalytic activity was also studied by continuously measuring the oxygen consumption in irradiated suspensions $\left(\mathrm{c}_{0, \text { phenol }}=0.5 \mathrm{mM}, \mathrm{c}_{\mathrm{TiO} 2}=200 \mathrm{mg} / \mathrm{L}\right)$. The biamperometric cell of the dissolved oxygen analyzer (Consort C835) was immersed in the sealed photoreactor, thus oxygen consumption could be monitored real-time during irradiation. The reactor was basically identical to the one used for general photocatalytic tests (Section 4.2.2), except it had two inlets in this case: one for air feedthrough and another to hold the oxygen electrode. During a typical experiment, the $\mathrm{TiO}_{2}$ suspension $(95 \mathrm{~mL})$ was filled into the reactor and then air was bubbled through it for about 10 minutes to reach the saturation level of $\mathrm{O}_{2}$. After equilibration under atmospheric pressure, the maximum achievable concentration of dissolved $\mathrm{O}_{2}$ was $8.25 \mathrm{ppm}$ at $25.0^{\circ} \mathrm{C}$. As soon 
as this value got stabilized, we quickly pipetted the phenol-containing solution $(5 \mathrm{~mL})$ and immediately sealed the system with a glass plug at the open joint. The reactor volume was almost exactly $100 \mathrm{~mL}$, only a negligible headspace $\left(\mathrm{V}_{\text {air }}<0.5 \mathrm{~mL}\right)$ remained. Finally, UV lamps were switched on and the monitoring of oxygen concentration started.

The oxygen electrode has been calibrated prior to each measurement with aerated high purity (brand MilliQ) water. The response time of the cell was also tested beforehand by keeping it in aerated MilliQ water until stable reading and then suddenly placing it into the same quality of water continuously purged with nitrogen. It was found that the displayed value dropped below $10 \%$ of the initial within 45 seconds. Based on this data, test conditions had to be optimized in order to slow down the rate of the oxygen consumption, otherwise it could not have been followed by our apparatus. The $\mathrm{TiO}_{2}$ content of suspensions was, therefore, decreased from the usual $1.0 \mathrm{~g} / \mathrm{L}$ (applied for aqueous photocatalytic tests) to $0.2 \mathrm{~g} / \mathrm{L}$.

\subsection{Applied materials}

Our reference photocatalysts (called P25-A and -B in the present work) were produced by the company Degussa. P25-B has recently been renamed to Aeroxide, however, it represents the same quality as P25-B. For the preparation of each type of solutions and suspensions, high purity MilliQ (Millipore) water was used. The $\mathrm{TiCl}_{4}$ precursor (Fluka) was of $99 \%$ purity. All other chemicals were purchased from Sigma-Aldrich and used without further purification (analytical grade materials). Dialysis of freshly synthesized $\mathrm{TiO}_{2}$ suspensions was made by the aid of Medicell dialysis sacks retaining molecules and particles bigger than 12000-14000 Dalton (average pore size). Gases used at synthesis runs and liquid phase experiments were delivered by Messer and they were of at least $99.9 \%$ purity. 


\section{Results and discussion}

\subsection{Flame synthesis first steps}

The development of a reliable and appropriately controllable flame synthesis method was a hard and effortful process with many dead-ends we encountered on the way to finally create BurnerA. The very first burners consisted of two perpendicular glass tubes molten together, which setup provided two inlets - one for the hydrogen and one for the air - and one outlet where premixed components were ignited. Although these types of burners were simple constructions, they turned out to have essential problems. Besides not offering the opportunity to independently adjust the precursor quantity, their smaller size and less sophisticated structure did not allow proper control over the main synthesis parameters either. The flames were often very unstable, thus incapable of ensuring reproducible syntheses. Material properties of the as-obtained catalysts have usually not correlated to the given key parameter actually adjusted, therefore, any detailed description of these experiments and related samples would be quite unreasonable to give.

Difficulties have also arisen when attempts were made to introduce the precursor via spraying by utilizing a concentric glass nebulizer. It was evident from the first time, that in lack of a professional (e.g., electronically controlled) flow regulator, the desired stable operation and hence homogeneous droplet size can hardly be achieved. Nevertheless, the main drawback of this technique was the limited range of applicable precursor materials. In order to produce pure $\mathrm{TiO}_{2}$ without introducing any unwanted contaminating/doping elements into the titania lattice, we have chosen $\mathrm{TiCl}_{4}$, which material has - in the end - turned out to be the most problematic one for atomization. Its fast hydrolysis led to an almost immediate clogging of the nozzle's capillary.

Even though the above "preliminary" studies consumed a lot of effort and time, they helped much in understanding the basic principles and limitations of flame-assisted $\mathrm{TiO}_{2}$ synthesis and in gathering experience with material characterization methods.

\subsection{Burner-A synthesis runs}

The design of Burner-A was finally proven to be a success as being an apparatus that allowed the production of titania powders in a controllable manner. The first important task was to experimentally determine the ideal parameter ranges - meaning basically the $\mathrm{H}_{2}$ and air flow rates where a stable flame with a height of at least a few centimeters could be generated. Its operational range was further confined by some limitations of the synthesis setup: the capabilities of the 
flowmeters as well as the pressure resistance of the silicone-rubber tubes (and their joints) used for driving the gases to the burner. In parallel, trials have been started with the bubble vapor generator filled with $\mathrm{TiCl}_{4}$, which construction - unlike the nebulizer applied before - could prevent serious clogging of the precursor intake line. Although a certain amount of hydrolysis product [a mixture of titanium oxide-hydroxide; $\left.\mathrm{TiO}_{\mathrm{x}}(\mathrm{OH})_{\mathrm{y}}\right]$ has often appeared in the precursor container, it was still not able to choke the tube. The amount of the precipitate could further be decreased by using air with highly reduced water content. As mentioned before, introducing the precursor in vapor form had the disadvantage of lower productivity compared to atomization (at least with our lab-scale apparatus) but, in return, it overcame technical limitations of the latter. It will be demonstrated that due to the well-controlled and continuous operation of the bubbler, $\mathrm{TiO}_{2}$ samples with relatively narrow size distribution could be obtained. In the present work, we name this characteristic "degree of dispersity" and quantify it by the FWHMs (full width at half maximum) of size distribution curves. These give values of 20-70 $\mathrm{nm}$.

Subsequent to the above necessary optimization, the first series of "real" experiments were conducted to explore how photocatalytic activity changes when adjusting the $\mathrm{H}_{2}-\mathrm{O}_{2}$ molar ratio within the interval of 0.67-1.21. Unfortunately, at this early stage of the study, the strategy of systematic parametrization was not appropriately followed. The "outer" air flow (i.e., the portion of air that remains from the total air stream after splitting a relatively small fraction for the precursor feed) was gradually decreased within a relatively broad range $(7470-4604 \mathrm{~mL} / \mathrm{min})$ while the precursor $(237$ $\mathrm{mL} / \mathrm{min})$ and the $\mathrm{H}_{2}$ flow rate $(1230 \mathrm{~mL} / \mathrm{min})$ were kept constant. This manner of adjustment - as it should have been foreseen - eventuates in simultaneous increase of both the particle residence times and the flame temperature. Consequently, one should observe a significant rise in rutile content and in particle sizes but results exhibit just the opposite this time. As seen in Figures 11, 12 and in Table 2 , both rutile and anatase sizes are rather decreasing and anatase content is slightly increasing with increasing $\mathrm{H}_{2}-\mathrm{O}_{2}$ molar ratio. 


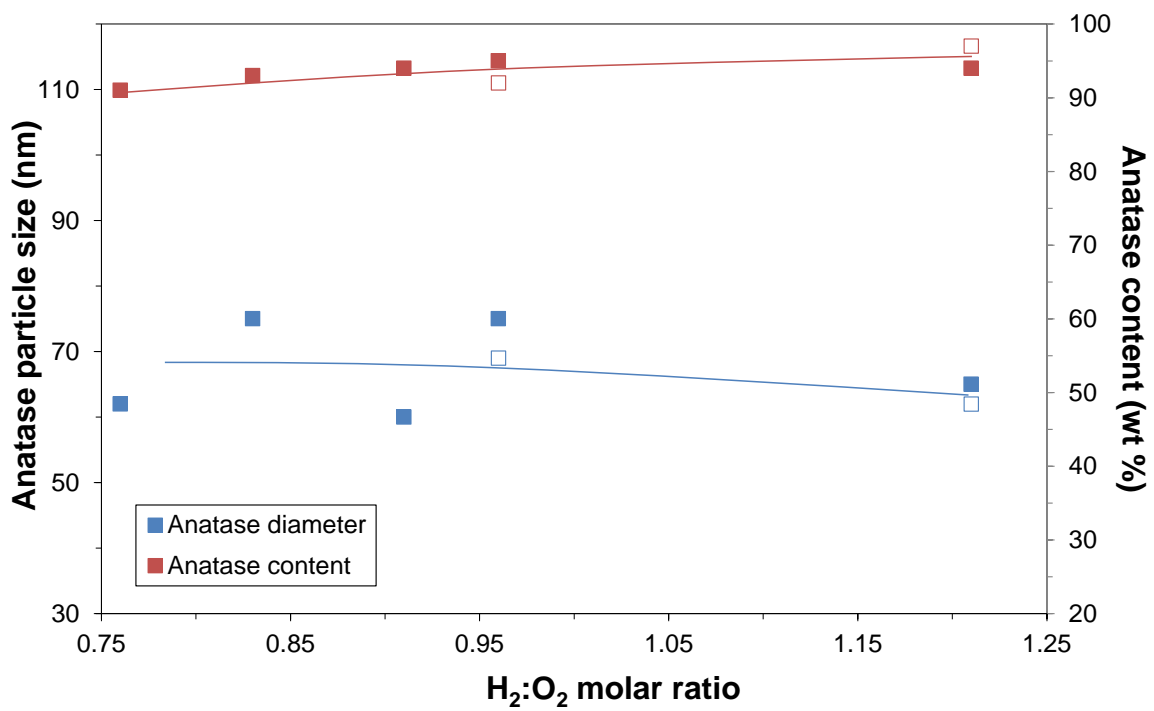

Figure 11: $\mathrm{H}_{2}-\mathrm{O}_{2}$ molar ratio dependency of anatase content and anatase particle size at Burner-A. Empty symbols represent samples originating from synthesis reproductions.

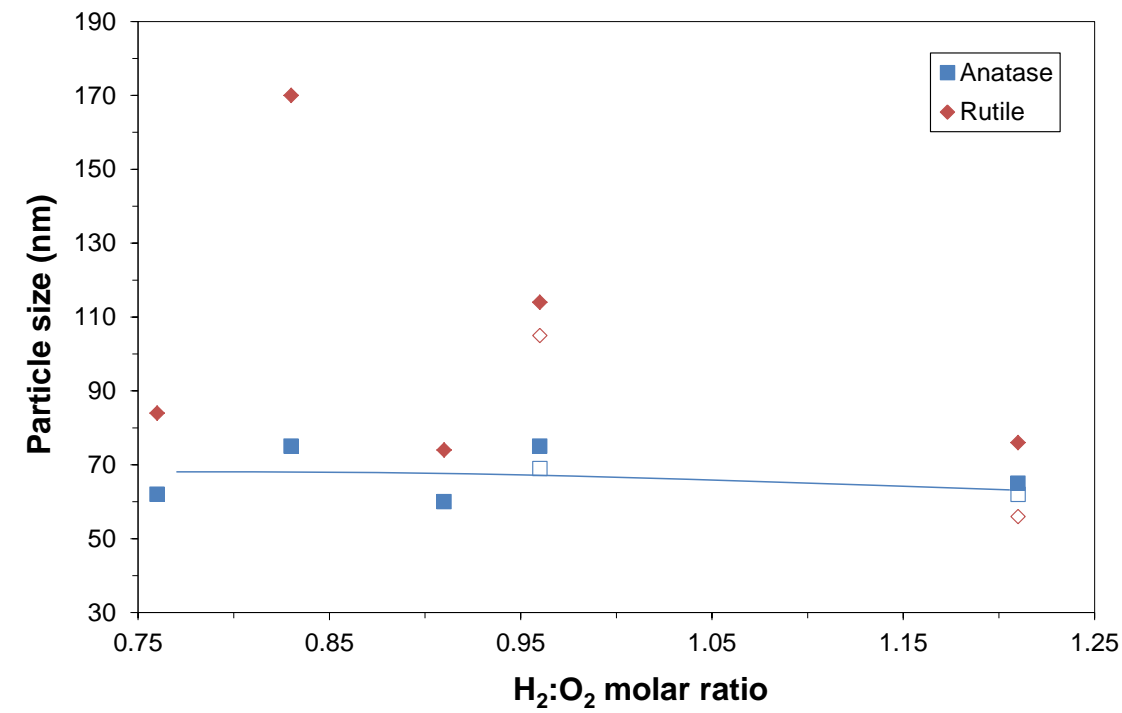

Figure 12: $\mathrm{H}_{2}-\mathrm{O}_{2}$ molar ratio dependency of both anatase and rutile particle sizes at Burner-A.

Empty symbols represent samples originating from synthesis reproductions.

\begin{tabular}{|c|c|c|c|c|c|c|}
\hline $\begin{array}{c}\text { Sample ID } \\
\text { (burner-H2: } \\
\text { VTiCl4- } \mathrm{T}_{\mathrm{TiCl}} \text { ) }\end{array}$ & $\begin{array}{l}\mathrm{H}_{2} \text { flow } \\
\text { rate } \\
(\mathrm{mL} / \mathrm{min})\end{array}$ & $\begin{array}{l}\text { Precursor } \\
\text { flow rate } \\
(\mathrm{mL} / \mathrm{min}) \\
\end{array}$ & $\begin{array}{c}\text { Outer air flow } \\
\text { rate } \\
(\mathrm{mL} / \mathrm{min})\end{array}$ & $\begin{array}{c}\mathrm{H}_{2} \\
\text { velocity } \\
(\mathrm{m} / \mathrm{min})\end{array}$ & $\begin{array}{l}\text { Precursor } \\
\text { velocity } \\
\text { (m/min) }\end{array}$ & $\begin{array}{l}\text { Outer air } \\
\text { velocity } \\
\text { (m/min) }\end{array}$ \\
\hline A-0.76-237-30 & 1230 & 237 & 7470 & 56.0 & 8.4 & 3.3 \\
\hline A-0.83-237-30 & 1230 & 237 & 6820 & 56.0 & 8.4 & 3.0 \\
\hline A-0.91-237-30 & 1230 & 237 & 6199 & 56.0 & 8.4 & 2.8 \\
\hline A-0.96-237-30 & 1230 & 237 & 5858 & 56.0 & 8.4 & 2.6 \\
\hline A-0.96-237-30/2 & 1230 & 237 & 5858 & 56.0 & 8.4 & 2.6 \\
\hline A-1.10-237-30 & 1230 & 237 & 5088 & 56.0 & 8.4 & 2.3 \\
\hline A-1.21-237-30 & 1230 & 237 & 4604 & 56.0 & 8.4 & 2.1 \\
\hline A-1.21-237-30/2 & 1230 & 237 & 4604 & 56.0 & 8.4 & 2.1 \\
\hline
\end{tabular}

Table 2: Gas flow rates used for the synthesis of A-samples produced by varying the $\mathrm{H}_{2}-\mathrm{O}_{2}$ molar ratio. 
The contradiction can only be resolved if one takes the nature of gas flow and linear gas velocities (calculated from the corresponding volumetric flow rates and the available flow cross-sectional areas; for deriving latter data, see Figure 3's caption) into account. Despite fluid dynamics was not investigated, it is reasonable to assume that the tangential inlet of the outer air will induce a quasiturbulent type flow inside the burner when the air collides into the stream-deflecting inner tubes/capillaries. The turbulence together with the angular momentum of the air stream - besides helping the mixing of the flame constituting elements - definitely exerts a perturbing influence on the $\mathrm{TiCl}_{4}$ flow, which most likely means an extended in-flame residence time of the precursor and of evolving particles. This reasoning becomes affirmable by considering the theoretically calculable residence time of a few tenth of a second, which can radically be altered through introducing only a little disturbance into the flow system. Heading towards higher $\mathrm{H}_{2}-\mathrm{O}_{2}$ molar ratios, the retentive effect is more and more hindered as the outer air flow rate decreases from the initial $7470 \mathrm{~mL} / \mathrm{min}$ to its lowest value of $4604 \mathrm{~mL} / \mathrm{min}$. The difference may seem even more drastic if we compare linear gas velocities calculated from the individual volumetric flows and the cross-sectional area of the respective burner orifices. It gives an initial 2.5-fold and a maximum 4.1-fold difference between the outer and the precursor carrier air velocities (Table 2). Consequently, applying the actual synthesis parameters created somewhat similar circumstances - apart from the flame temperature change - to that, when the precursor flow is gradually increased while keeping the $\mathrm{H}_{2}-\mathrm{O}_{2}$ molar ratio and the overall gas flow rate constant. The fluctuation in particle sizes is most probably reflecting the simultaneous action of the above-described two opposing synthesis parameters (flame temperature and residence time), when they are alternately overwhelming each other. The correctness of data points is incidentally justified by the acceptable level of reproducibility, represented in figures by full-empty symbol pairs belonging to the same value of the actual independent variable.

\begin{tabular}{|c|c|c|c|c|c|}
\hline $\begin{array}{c}\text { Sample ID } \\
\text { (burner-H }{ }_{2}: \mathrm{O}_{2}- \\
\text { vTiCl4- }_{\mathrm{TiCl} 4} \text { ) }\end{array}$ & $\begin{array}{c}\text { Anatase } \\
\text { content } \\
(w t \%)\end{array}$ & $\begin{array}{c}\text { Anatase } \\
\text { size / XRD } \\
(\mathrm{nm})\end{array}$ & $\begin{array}{l}\text { Rutile size / } \\
\text { XRD (nm) }\end{array}$ & $\begin{array}{l}\text { Particle size } \\
\text { / TEM (nm) }\end{array}$ & $\begin{array}{c}\text { Degree of } \\
\text { dispersity } \\
\text { / TEM (nm) }\end{array}$ \\
\hline A-0.76-237-30 & 91 & 62 & 84 & 53 & 42 \\
\hline A-0.83-237-30 & 93 & 71 & 170 & 60 & 40 \\
\hline A-0.91-237-30 & 94 & 60 & 74 & - & - \\
\hline A-0.96-237-30 & 92 & 69 & 105 & 51 & 38 \\
\hline A-0.96-237-30/2 & 95 & 75 & 114 & 52 & 38 \\
\hline A-1.10-237-30 & - & - & - & - & - \\
\hline A-1.21-237-30 & 94 & 65 & 76 & 57 & 48 \\
\hline A-1.21-237-30/2 & 97 & 62 & 56 & 63 & 40 \\
\hline
\end{tabular}

Table 3: Structural parameters of A-samples produced by varying the $\mathrm{H}_{2}-\mathrm{O}_{2}$ molar ratio. Data for sample A-1.10-237-30 are not available.

It shall be mentioned here, that as soon as we realized the importance of altering only one parameter at a time, we laid special emphasis on more careful design of all further experiments with 
Burner-A as well as with Burner-B. Unfortunately, we were not able to resynthesize the above series of samples with Burner-A due to its later unrepairable damage (explained later in more details). The same applies to sample A-1.10-237-30 whose quantity was enough only for having aqueous photocatalytic runs executed, but for nothing more.

Another apparent discrepancy emerges when looking at the experimental results obtained with fixed $\mathrm{H}_{2}-\mathrm{O}_{2}$ molar ratio. Although synthesis parameters were appropriately set up this time, - the precursor carrier air flow rate was adjusted alone - resulting $\mathrm{TiO}_{2}$ characteristics varied again just contrarily to the expectations (Figure 13 and 14). Particle diameters should have decreased and the anatase content should have increased as the precursor flow rate increased because particles have spent less and less time in the flame. Interestingly, rutile size was the only parameter that changed so as anticipated.

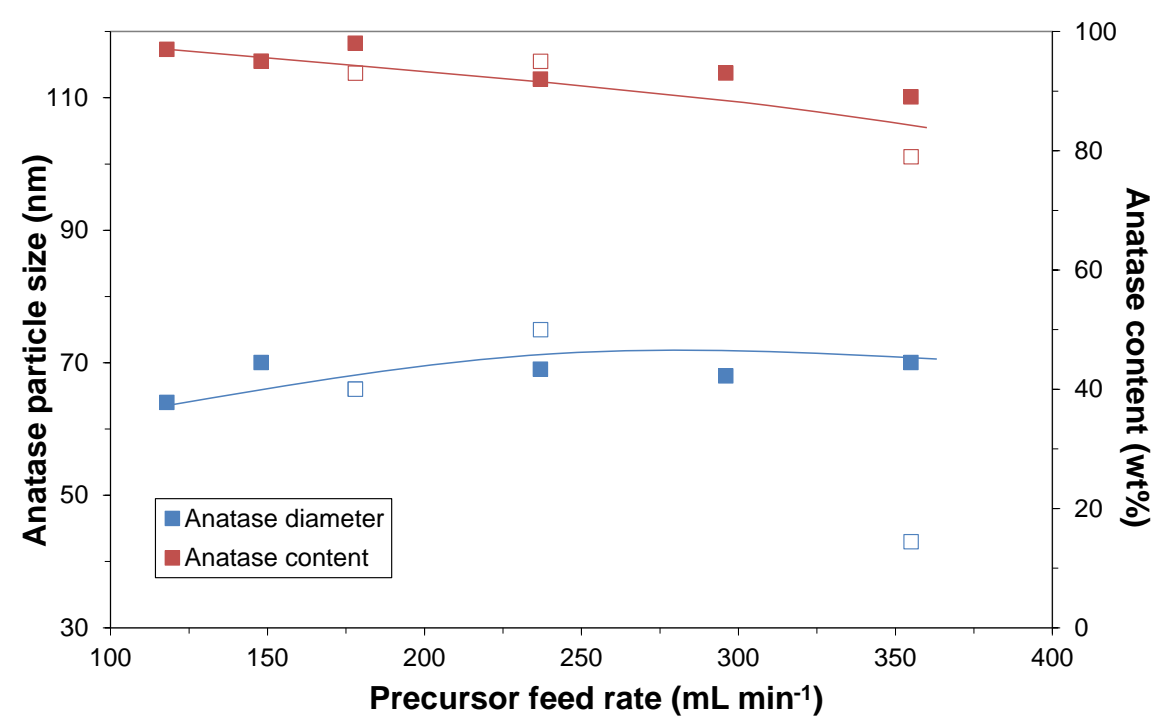

Figure 13: $\mathrm{TiCl}_{4}$ feed rate dependency of anatase content and anatase particle size at Burner-A. Empty symbols represent samples originating from synthesis reproductions.

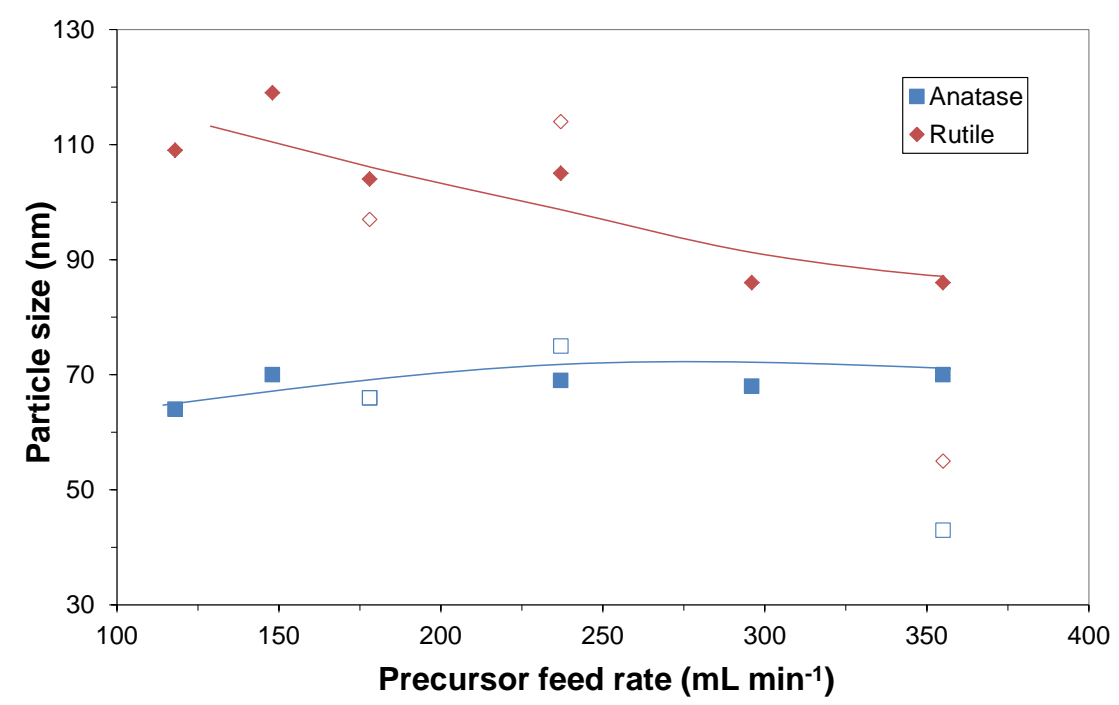

Figure 14: $\mathrm{TiCl}_{4}$ feed rate dependency of both anatase and rutile particle sizes at Burner-A. Empty symbols represent samples originating from synthesis reproductions. 
Unfortunately, in lack of some essential data (like accurate flame temperature distribution and flow characteristics) the interpretation of the current observations can solely be laid on more hypothetical basis. It seems that simultaneous interplay of numerous factors shall be taken into account to render a reason for the improbable change of sample properties. First of all, we must assume nearly unchanged temperature distribution and flame geometry throughout this experimental series, since the $\mathrm{H}_{2}$ flow was set constant and the outer air flow was only slightly reduced by splitting increasingly bigger portions for precursor feed (Table 4).

\begin{tabular}{|c|c|c|c|c|c|c|}
\hline $\begin{array}{c}\text { Sample ID } \\
\text { (burner-H } \\
\text { vTiCl4-T }: \mathrm{O}_{2} \text { - } \\
\text { TiCl4) }\end{array}$ & $\begin{array}{l}\mathrm{H}_{2} \text { flow } \\
\text { rate } \\
(\mathrm{mL} / \mathrm{min})\end{array}$ & $\begin{array}{l}\text { Precursor } \\
\text { flow rate } \\
(\mathrm{mL} / \mathrm{min})\end{array}$ & $\begin{array}{l}\text { Outer air flow } \\
\text { rate } \\
(\mathrm{mL} / \mathrm{min})\end{array}$ & $\begin{array}{c}\mathbf{H}_{2} \text { velocity } \\
\text { (m/min) }\end{array}$ & $\begin{array}{l}\text { Precursor } \\
\text { velocity } \\
\text { (m/min) }\end{array}$ & $\begin{array}{l}\text { Outer air } \\
\text { velocity } \\
\text { (m/min) }\end{array}$ \\
\hline A-0.96-118-30 & 1230 & 118 & 5977 & 56.0 & 4.2 & 2.7 \\
\hline A-0.96-148-30 & 1230 & 148 & 5947 & 56.0 & 5.2 & 2.7 \\
\hline A-0.96-178-30 & 1230 & 178 & 5917 & 56.0 & 6.3 & 2.6 \\
\hline$A-0.96-178-30 / 2$ & 1230 & 178 & 5917 & 56.0 & 6.3 & 2.6 \\
\hline A-0.96-237-30 & 1230 & 237 & 5858 & 56.0 & 8.4 & 2.6 \\
\hline $\mathrm{A}-0.96-237-30 / 2$ & 1230 & 237 & 5858 & 56.0 & 8.4 & 2.6 \\
\hline A-0.96-296-30 & 1230 & 296 & 5799 & 56.0 & 10.5 & 2.6 \\
\hline A-0.96-355-30 & 1230 & 355 & 5740 & 56.0 & 12.6 & 2.6 \\
\hline $\mathrm{A}-0.96-355-30 / 2$ & 1230 & 355 & 5740 & 56.0 & 12.6 & 2.6 \\
\hline
\end{tabular}

Table 4: Gas flow rates used for the synthesis of A-samples produced by varying the precursor feed.

\begin{tabular}{|c|c|c|c|c|c|}
\hline $\begin{array}{c}\text { Sample ID } \\
\text { (burner-H } \\
\text { vTiCl4-T }_{\text {TiCl4 }} \text { ) }\end{array}$ & $\begin{array}{l}\text { Anatase } \\
\text { content } \\
\text { (wt \%) }\end{array}$ & $\begin{array}{l}\text { Anatase size } \\
\text { / XRD (nm) }\end{array}$ & $\begin{array}{l}\text { Rutile size / } \\
\text { XRD }(\mathrm{nm})\end{array}$ & $\begin{array}{l}\text { Particle size } \\
\text { / TEM }(\mathrm{nm})\end{array}$ & $\begin{array}{c}\text { Degree of } \\
\text { dispersity } \\
\text { / TEM (nm) }\end{array}$ \\
\hline A-0.96-118-30 & 97 & 64 & 109 & 54 & 38 \\
\hline \begin{tabular}{|l|}
$A-0.96-148-30$ \\
\end{tabular} & 95 & 70 & 119 & 60 & 50 \\
\hline A-0.96-178-30 & 98 & 66 & 104 & 62 & 42 \\
\hline $\mathrm{A}-0.96-178-30 / 2$ & 93 & 66 & 97 & 51 & 46 \\
\hline A-0.96-237-30 & 92 & 69 & 105 & 51 & 38 \\
\hline $\mathrm{A}-0.96-237-30 / 2$ & 95 & 75 & 114 & 52 & 38 \\
\hline A-0.96-296-30 & 93 & 68 & 86 & 58 & 40 \\
\hline A-0.96-355-30 & 89 & 70 & 86 & 55 & 40 \\
\hline $\mathrm{A}-0.96-355-30 / 2$ & 79 & 43 & 55 & 48 & 42 \\
\hline
\end{tabular}

Table 5: Structural parameters of A-samples produced by varying the precursor feed rate.

Consequently, the factor which may reasonably explain the unexpected variations of product properties is the precursor concentration in the gas phase. It is evident that by increasing the volumetric flow of the $\mathrm{TiCl}_{4}$ carrier branch the bubbling in the liquid precursor will intensify. This, in turn, generates a larger liquid-air interface through which a higher rate of evaporation will be provided. In parallel, the number of those small precursor droplets that are released by bubbles' burst is also growing and their evaporation further increases the gas phase concentration of $\mathrm{TiCl}_{4}$. Even though the concentration cannot be elevated above its equilibrium level specific to the given temperature, the continuously increasing precursor supply may effectively compensate the enhanced 
dilution effect of the air stream when setting higher and higher volumetric flows. Provided that a comparable precursor concentration is sustained in the flame at each precursor feed rate, the collision rate of the molecules and nascent particles should also remain very similar. This assumption is supported by TEM particle size distribution data showing a relatively stable dispersity value of $c a$. $40 \mathrm{~nm}$, all along the series (Table 5 and Figure 15). In case of a gradually decreasing gas phase $\mathrm{TiCl}_{4}$ concentration the degree of dispersity would inevitably drop, while more concentrated reaction mixtures would yield $\mathrm{TiO}_{2} \mathrm{~s}$ with more diverse particle sizes.
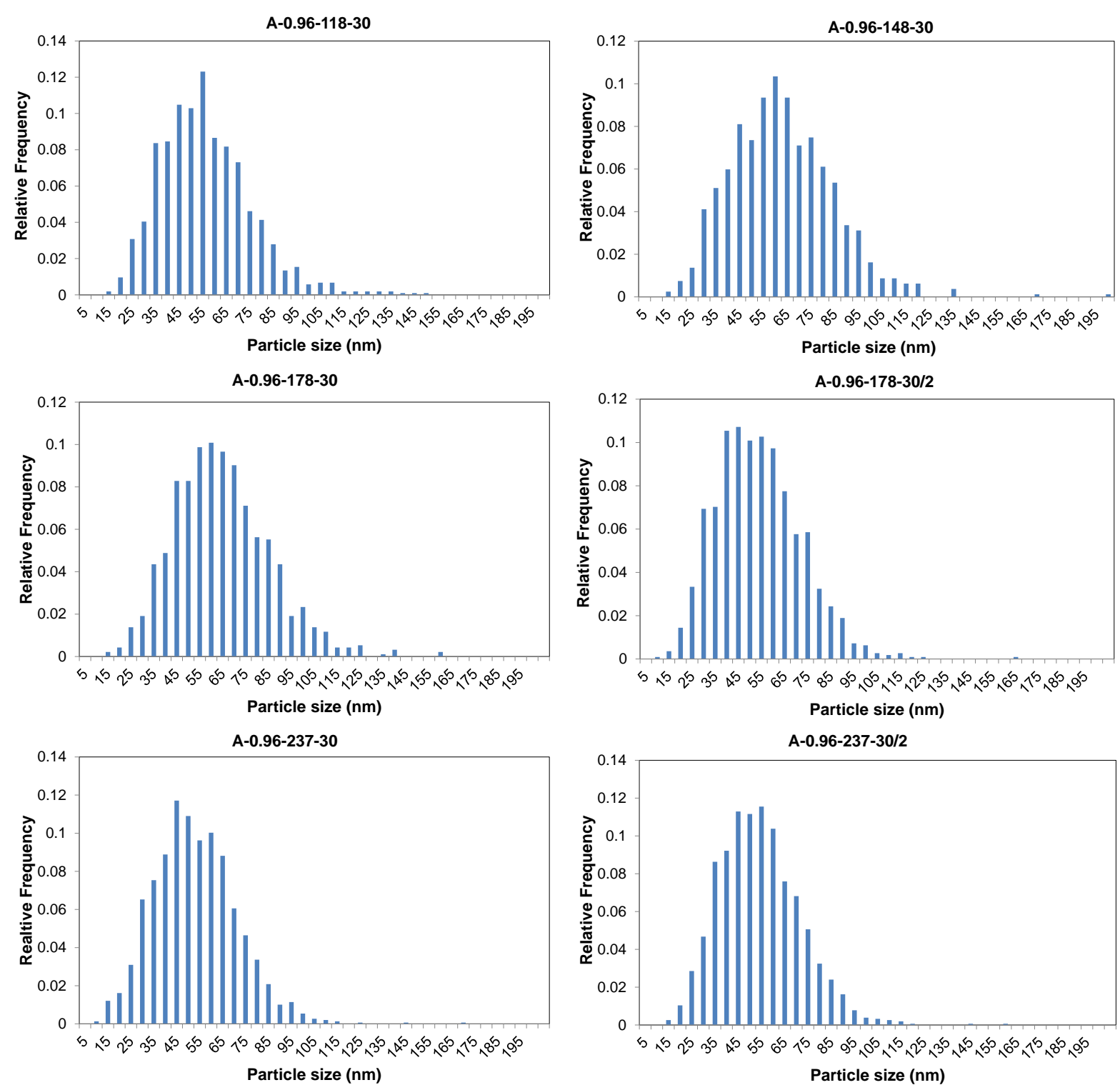

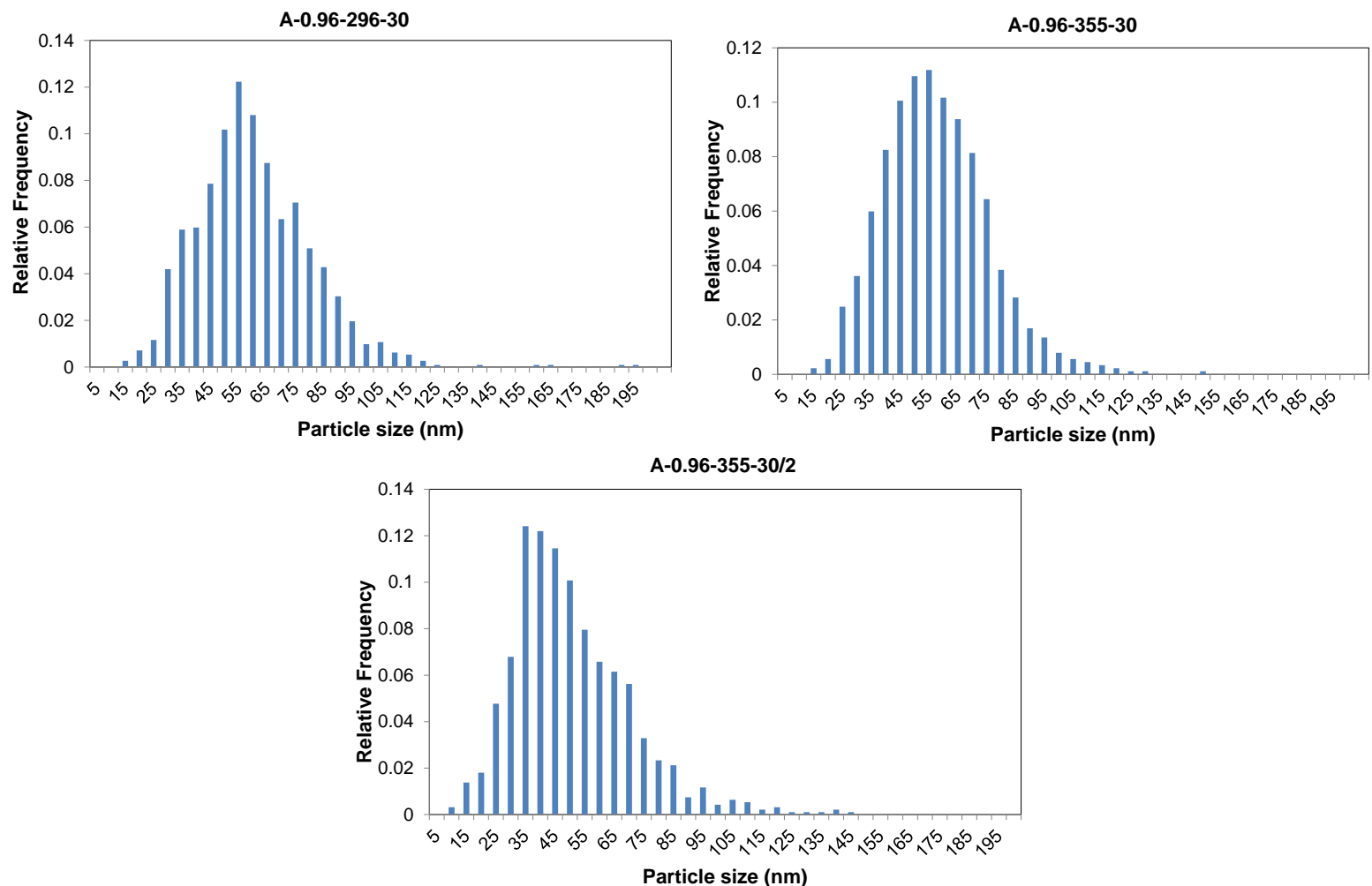

Figure 15: TEM particle size distribution histograms of A-samples produced by varying the precursor feed rate. Slight change is seen in the average particle sizes while the degree of dispersity stays more or less constant within this series.

Resuming the above deduction, there is no other logical explanation left but a certain extent of flame temperature increase, which resulted in bigger anatase grain size and higher rutile content. Since the $\mathrm{H}_{2}-\mathrm{O}_{2}$ ratio was unchanged within the series only the $\mathrm{TiO}_{2}$ formation could introduce additional energy into the reaction system. Even though we predict a relatively constant precursor concentration at each flow rate, the amount of $\mathrm{TiCl}_{4}$ transformed into titania per unit volume and per unit time will increase as the flow rate grows. Accordingly, the enthalpy content and the temperature of the flame - at least locally, within and in the close proximity of the reaction zone where $\mathrm{TiO}_{2}$ is formed - will become higher and higher. Nevertheless, there shall be a point beyond which the evaporation rate cannot keep up with the precursor carrier flow increase, therefore the $\mathrm{TiCl}_{4}$-mixture starts becoming leaner. This turn may occur somewhere above the precursor flow rate of $355 \mathrm{~mL} / \mathrm{min}$ as it is proven by sample A-0.96-355-30/2. Here, the exact precursor feed rate is uncertain but it can be taken for granted that it reached a higher value than $355 \mathrm{~mL} / \mathrm{min}$, as explained in the next paragraph.

It was already mentioned before that Burner-A has continuously suffered from a heat induced deformation of the disposable outer precursor feeding tube due to the closeness of the high temperature zone. This structural change practically meant the melting of the tube's orifice which then shrank to a smaller diameter. Yet, this narrowing could not have any significant effect on sample properties (proven by numerous synthesis reproductions) because it occurred in a very short time 
relative to burner ignition and the extent of shrinkage was always very similar. Incidentally, after each synthesis run the deformed auxiliary tube was replaced by a new one. However, the fixed inner precursor feeding tube has unfortunately got narrowed as well during the last few experiments which led to the ultimate and unrepairable damage of the burner. The root cause was the clogging of the tube triggered by the deposition of a thermally decomposed doping precursor when attempts were made to synthesize Fe-containing $\mathrm{TiO}_{2}$ catalysts. Sample A-0.96-355-30/2 was the only one out of the entire set of A-samples that has been fabricated by reduced-diameter inner tube. It is obvious that narrowing the tube is accompanied by a higher velocity of precursor molecules and the reduced residence time then leads to a drastic drop in both anatase and rutile particle sizes. The only perplexing observation on material properties of sample A-0.96-355-30/2 is that its anatase content has also decreased compared to sample A-0.96-355-30. This phenomenon is inconsistent with the significant decrease of both particle sizes and cannot be resolved on the basis of those particle formation rules that have been proven to be applicable to our system.

By taking the foregoing elucidation into account, the continuously decreasing tendency of rutile particle size also becomes explicable. It is somewhat contradictory at first glance, that rutile size is decreasing while anatase particle sizes are getting bigger and rutile content is increasing as well. This, however, seems not that extraordinary anymore if we assume the following process valid: by increasing the precursor flow rate the flame temperature starts slightly increasing which intensifies particle growth as well as phase transformation. Rutile phase appears in the product aerosol once the grain size and the temperature (i.e., the amount of energy that is transferred to the solid material system) reached a certain threshold value. Simultaneously, the in-flame residence time of the solid matter is getting shorter as the precursor flow rate increases, therefore nascent rutile particles have less and less time to grow while their number is still increasing and anatase particles are growing further as well. Then when the flow rate arrives to the value beyond which the reaction mixture will gradually be leaner and hence the temperature does not increase further (moreover, it presumably decreases as the air carries more and more heat away from the reaction zone), rutile formation will be pushed back and anatase particle size starts to decrease as well. Rutile grain sizes will - of course - keep further decreasing. Unfortunately, this turn-point could not be reached by Burner-A due to its damage, but the trends observed in B-samples' material properties can suitably support the validity of the above theory.

All in all, it was pointed out that within both experimental series of Burner-A parameter adjustments, the considerably large fluctuation of particle diameters to sequential data points and the unexpected trends experienced in material characteristics can equally be broken down to the continuous competition of the two key synthesis parameters: flame temperature and in-flame residence time. It was a matter of luck that such special circumstances have been established which 
enabled us to discover these basic correlations. In case of $\mathrm{H}_{2}-\mathrm{O}_{2}$ molar ratio change the improper design of experiment, while for precursor feed rate adjustment the investigated parameter range itself revealed the underlying mechanisms of particle formation. It will be shown later, that at Burner-B synthesis runs the former "problem" was completely eliminated and the precursor flow rate was altered in a too wide interval (coupled with slightly different burner geometry and operational parameters) to attract the attention alone to the previously described subtle irregularities appearing only at the lowest values of the flow range.

\subsection{Photocatalytic activity of A-samples}

Simultaneously to synthesis runs, photocatalytic activities were determined via phenol decomposition model reactions in order to see how catalysts' performances vary with altering the production parameters. Naturally, the ultimate goal was to find correlation between material properties and photocatalytic efficiencies, and by utilizing this knowledge, to reach the highest possible activity through fine-tuning the flame synthesis process.

Accordingly, we first investigated the dependency between photocatalytic activities and two basic $\mathrm{TiO}_{2}$ characteristics: particle size and phase composition. For the former, by default, anatase particle sizes (derived from XRD data) were always considered, as this phase was the predominant constituent of nearly all samples and it was reported in the literature to display activity much superior to rutile's. It was interesting to see, though, that there is apparently no clear interdependence between these properties and photocatalytic activities at any of the two experimental series. When altering the $\mathrm{H}_{2}-\mathrm{O}_{2}$ molar ratio (Figure 16, Table 6), crystallite sizes vary within a ca. $20 \mathrm{~nm}$ wide interval showing a slight overall decrease, while anatase content rather monotonously increases only by 6\%, (from 91 to $97 \mathrm{wt} \%)$. Despite these small changes in material characteristics, photocatalytic activity ( $\mathrm{r}_{0, \text { phenol }}$ ) shows a 2.6-fold variation between the lowest and the highest values. An even more conspicuous example is represented by samples A-1.21-237-30 and A-0.91-237-30. Even though they practically have the same anatase content and only a $5 \mathrm{~nm}$ difference in their average anatase diameter, the photocatalytic activity of sample A-0.91-237-30 is 2.4-times higher than that of 1.21-237-30. For precursor feed rate change series this tendency is a bit less emphasized but it is still there (Figure 17, Table 6). 


\begin{tabular}{|c|c|c|c|}
\hline $\begin{array}{c}\text { Sample ID } \\
\text { (burner- } \mathrm{H}_{2}: \mathrm{O}_{2} \text { - } \\
\text { VTiCl4-TTicl4) }\end{array}$ & $\begin{array}{c}\text { Anatase } \\
\text { content } \\
(w t \%)\end{array}$ & $\begin{array}{c}\text { Anatase } \\
\text { size / XRD } \\
(\mathbf{n m})\end{array}$ & $\begin{array}{l}\text { Activity/ro,phenol } \\
\left(\times 10^{-8} \mathrm{Ms}^{-1}\right)\end{array}$ \\
\hline A-0.76-237-30 & 91 & 62 & 9.80 \\
\hline A-0.83-237-30 & 93 & 71 & 11.00 \\
\hline A-0.91-237-30 & 94 & 60 & 16.10 \\
\hline A-0.96-237-30 & 92 & 69 & 15.90 \\
\hline A-0.96-237-30/2 & 95 & 75 & 15.60 \\
\hline A-1.10-237-30 & - & - & 17.70 \\
\hline A-1.21-237-30 & 94 & 65 & 6.72 \\
\hline A-1.21-237-30/2 & 97 & 62 & 7.20 \\
\hline A-0.96-118-30 & 97 & 64 & 12.80 \\
\hline A-0.96-148-30 & 95 & 70 & 10.90 \\
\hline A-0.96-178-30 & 98 & 66 & 11.30 \\
\hline $\mathrm{A}-0.96-178-30 / 2$ & 93 & 66 & 11.60 \\
\hline A-0.96-237-30 & 92 & 69 & 15.90 \\
\hline A-0.96-237-30/2 & 95 & 75 & 15.60 \\
\hline A-0.96-296-30 & 93 & 68 & 14.90 \\
\hline A-0.96-355-30 & 89 & 70 & 20.20 \\
\hline A-0.96-355-30/2 & 79 & 43 & 24.20 \\
\hline
\end{tabular}

Table 6: Structural parameters and photoactivities of all A-samples. Former data of sample A1.10-237-30 are not available. 

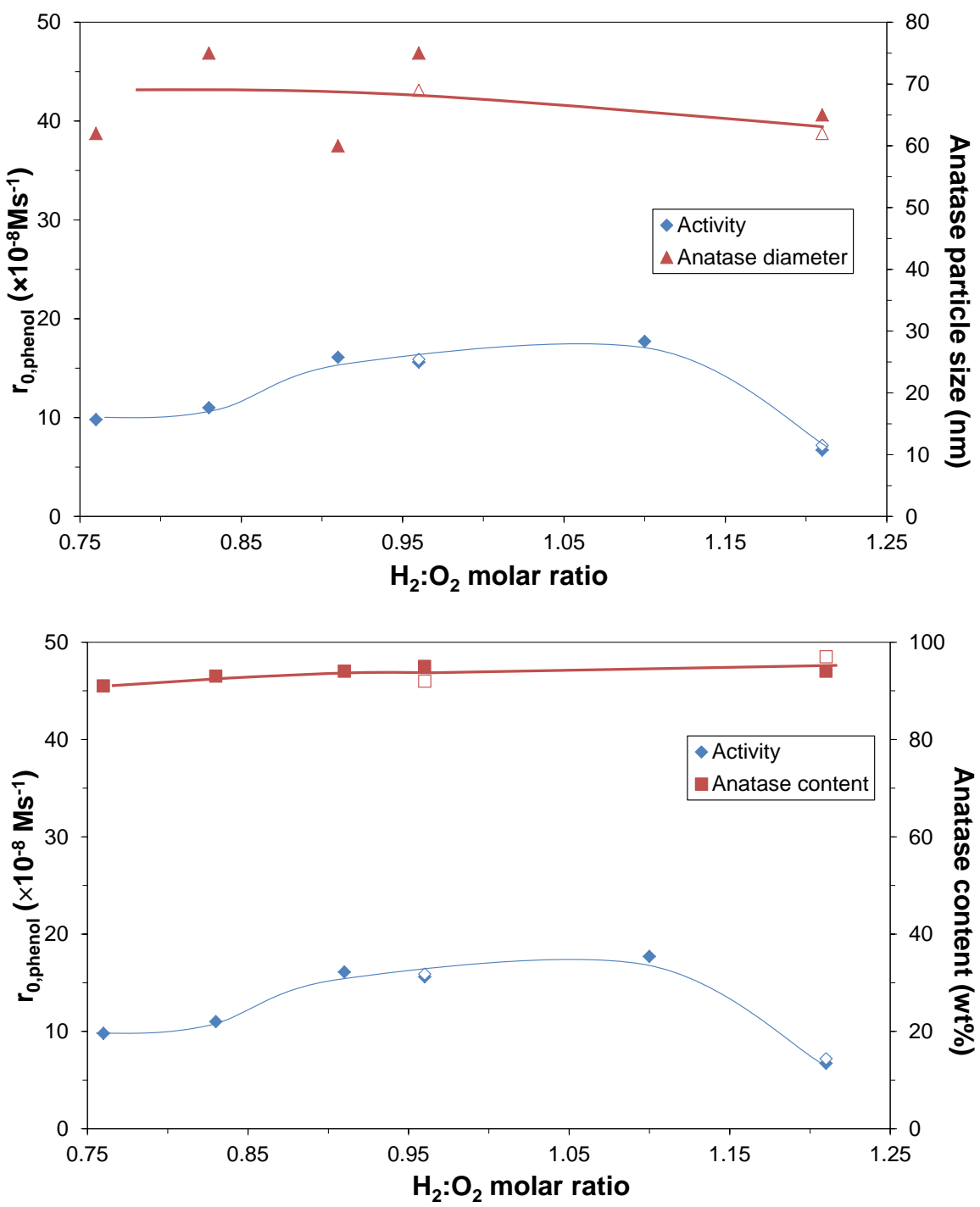

Figure 16: $\mathrm{H}_{2}-\mathrm{O}_{2}$ molar ratio dependency of anatase content, particle size and photocatalytic activity at Burner-A. Empty symbols represent samples originating from synthesis reproductions. 

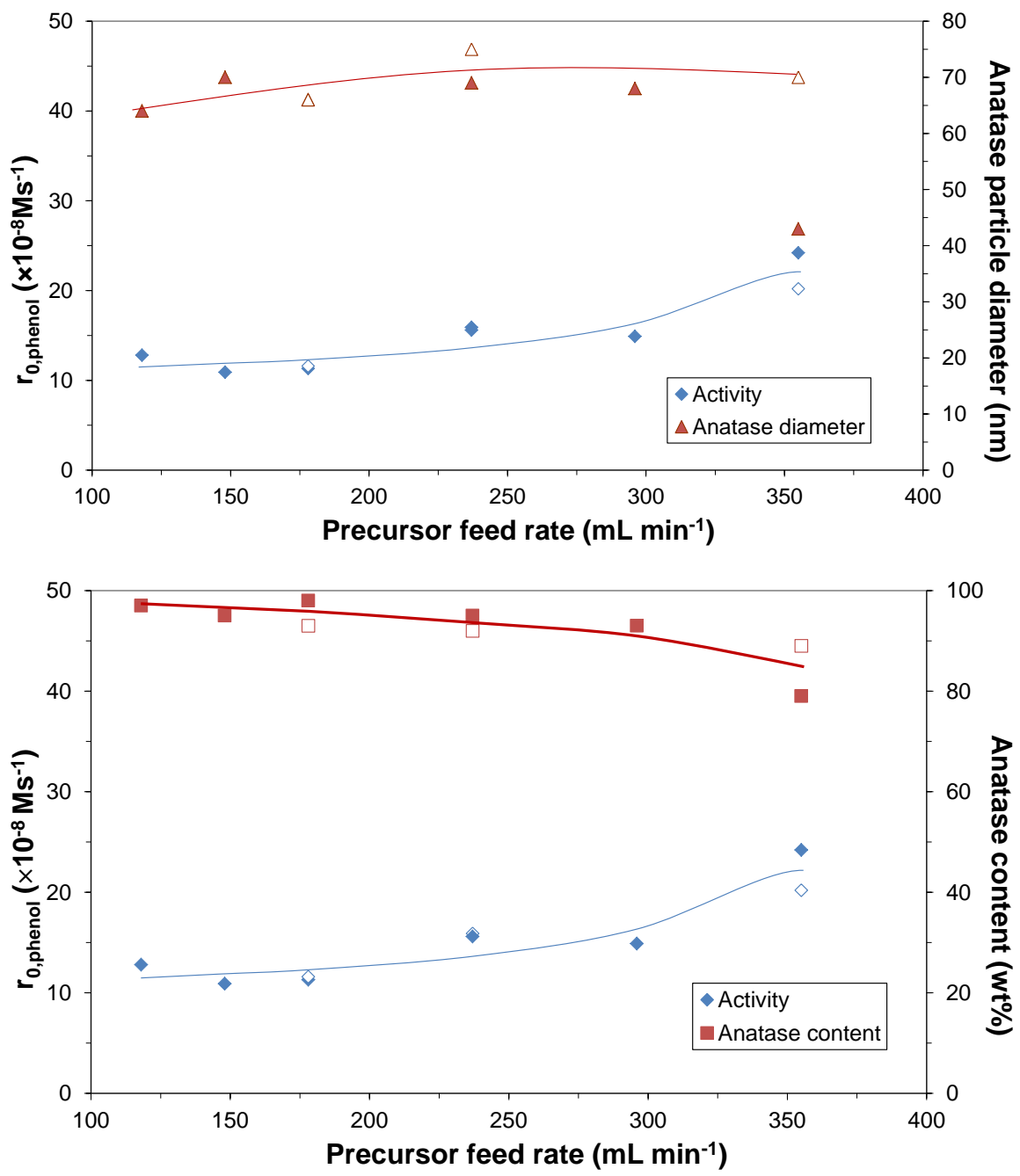

Figure 17: $\mathrm{TiCl}_{4}$ feed rate dependency of anatase content, particle size and photocatalytic activity at Burner-A. Empty symbols represent samples originating from synthesis reproductions. 
To properly visualize the interrelation of parameters just discussed, photocatalytic activities of all A-samples are plotted against the phase composition and the anatase size in Figure 18.
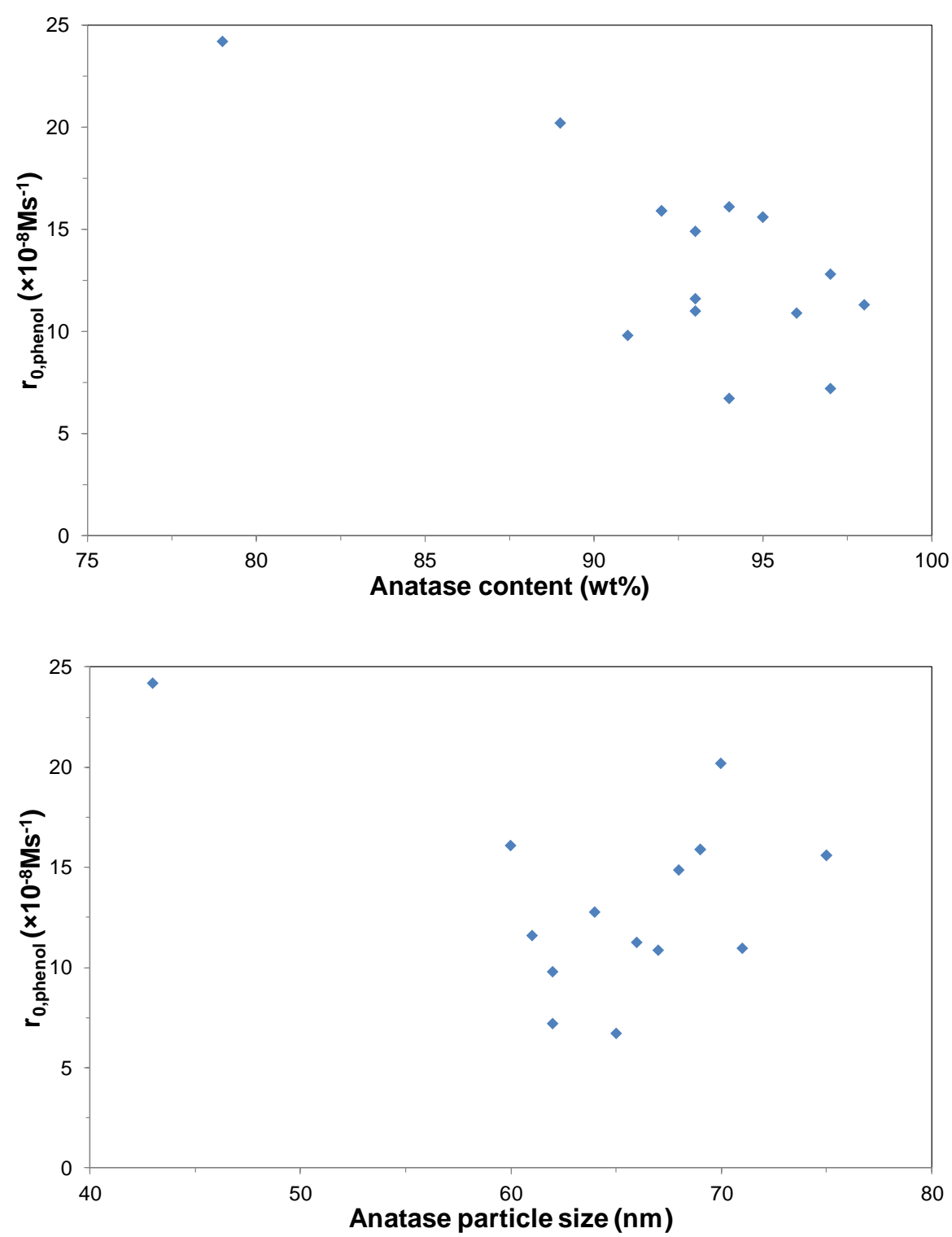

Figure 18: Photocatalytic activities of A-samples are shown as a function of anatase particle size and phase composition.

It appears from these representations that there is no unambiguous correlation between $\mathrm{r}_{0}$ and the basic material properties as data points scatter remarkably. Then the question may arise: what can cause such a large variation of photoactivities? The most attractive explanation was found when TEM images have been evaluated. We discovered that a well-observable connection exists between particle shape and $\mathrm{r}_{0}$. Samples with the smallest photoactivity contain predominantly spherical particles, while those with the largest $\mathrm{r}_{0}$-s consist almost exclusively of polyhedral (faceted) ones. This interrelation is demonstrated the best by images in Figure 19, which show TEM projections of samples A-1.21237-30/2 and A-0.96-355-30. The former displayed one of the lowest activities whereas the latter possesses the second highest amongst A-samples. To make this shape parameter numerically 
expressible, the following classification was made: a particle was defined to be polyhedral, if it was seen to be terminated mostly by linear sections rather than by arcs on TEM projections. Owing to the carefully designed methodology of TEM sampling and evaluation (explained earlier at TEM measurement description), we obtained sufficiently representative statistics on particle morphology as well. Its A-sample results are shown in Table 7 along with photoactivities and with the main material properties. As previously mentioned, not all samples were investigated by TEM, therefore a few morphology data will be missing in the further evaluation of measurement results.

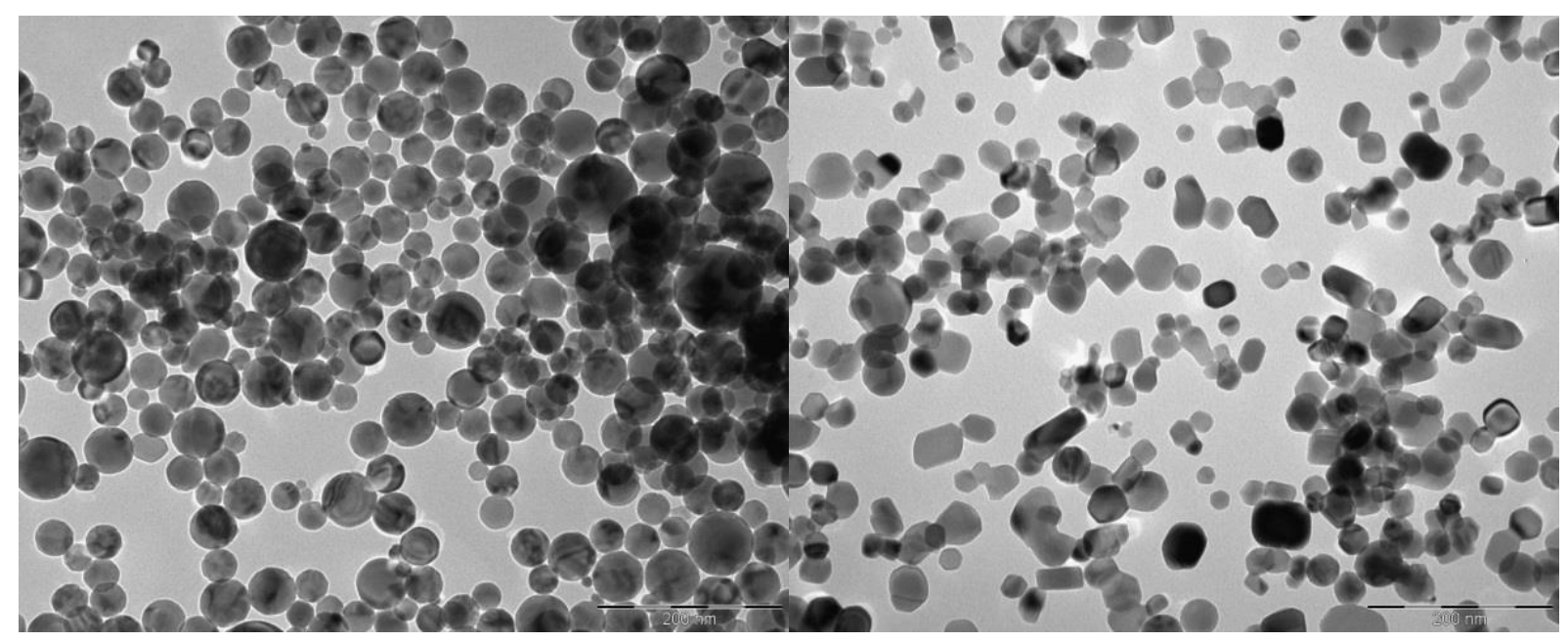

Figure 19: TEM images of sample A-1.21-237-30/2 (left) and A-0.96-355-30 (right). Both scale bars - located in the lower right corners of the images - represent a length of $200 \mathrm{~nm}$.

\begin{tabular}{|c|c|c|c|c|}
\hline $\begin{array}{c}\text { Sample ID } \\
\text { (burner-H } \mathrm{H}_{2}: \mathrm{O}_{2}- \\
\text { VTiCl4- }_{\text {TiCl4 }} \text { ) }\end{array}$ & $\begin{array}{c}\text { Anatase } \\
\text { content } \\
(w t \%)\end{array}$ & $\begin{array}{c}\text { Anatase } \\
\text { size / XRD } \\
(\mathbf{n m})\end{array}$ & $\begin{array}{l}\text { Activity/ro,phenol } \\
\left(\times 10^{-8} \mathrm{Ms}^{-1}\right)\end{array}$ & $\begin{array}{c}\text { Frequency of } \\
\text { polyhedral } \\
\text { particles }(\%)\end{array}$ \\
\hline A-0.76-237-30 & 91 & 62 & 9.80 & 60 \\
\hline A-0.83-237-30 & 93 & 71 & 11.00 & 59 \\
\hline A-0.91-237-30 & 94 & 60 & 16.10 & - \\
\hline A-0.96-237-30 & 92 & 69 & 15.90 & 59 \\
\hline A-0.96-237-30/2 & 95 & 75 & 15.60 & 49 \\
\hline A-1.10-237-30 & - & - & 17.70 & - \\
\hline A-1.21-237-30 & 94 & 65 & 6.72 & 47 \\
\hline $\mathrm{A}-1.21-237-30 / 2$ & 97 & 62 & 7.20 & 0 \\
\hline A-0.96-118-30 & 97 & 64 & 12.80 & 48 \\
\hline A-0.96-148-30 & 95 & 70 & 10.90 & 10 \\
\hline A-0.96-178-30 & 98 & 66 & 11.30 & 33 \\
\hline A-0.96-178-30/2 & 93 & 66 & 11.60 & 78 \\
\hline A-0.96-237-30 & 92 & 69 & 15.90 & 59 \\
\hline A-0.96-237-30/2 & 95 & 75 & 15.60 & 49 \\
\hline A-0.96-296-30 & 93 & 68 & 14.90 & 54 \\
\hline A-0.96-355-30 & 89 & 70 & 20.20 & 69 \\
\hline $\mathrm{A}-0.96-355-30 / 2$ & 79 & 43 & 24.20 & 99 \\
\hline P25-A & 79 & 27 & 11.80 & 85 \\
\hline P25-B & 89 & 27 & 15.40 & 100 \\
\hline
\end{tabular}

Table 7: Structural parameters, photoactivities and shape statistics of all A-samples and P25 catalysts. 
The activity-shape correlation is revealed by Figure 20, where $\mathrm{TiO}_{2} \mathrm{~s}$ originating from the two different synthesis series are plotted with different color. Apart from some scattering points, the tendency of having higher photoactivity with more polyhedral grains present in the samples seems plausible. We deem this theory being double-confirmed by the P25 catalysts amongst which the one containing more polyhedral particles (named P25-B) has displayed 30\% higher photoactivity under the same experimental conditions. As a matter of fact, P25-B has 10\% higher anatase content but has the same particle size than that of P25-A. However, this extent of composition difference can equally be found amongst our flame-made $\mathrm{TiO}_{2} \mathrm{~S}$ where the impact of crystalline phase ratio on photoactivity was appeared to be indecisive.

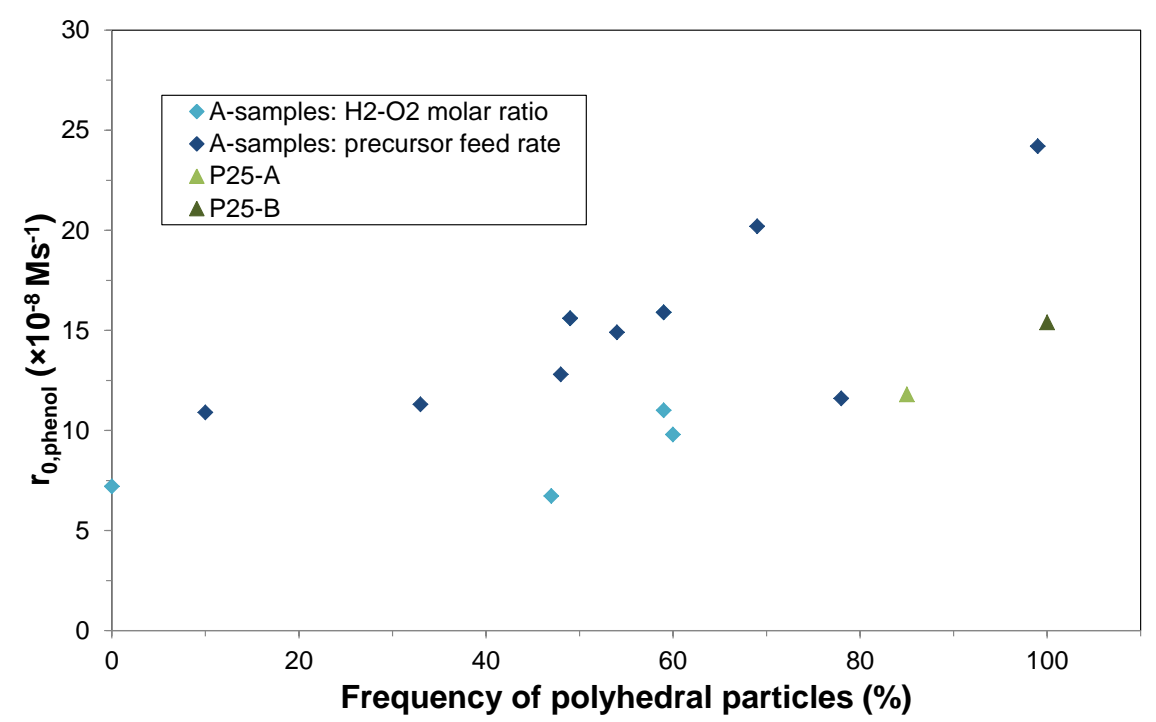

Figure 20: Initial phenol decomposition rates plotted as a function of polyhedral particle content.

The key role of particle morphology is well-known in heterogeneous catalytic processes as crystal facet-dependent catalytic activity has been pointed out many times [221-223]. Specifically to $\mathrm{TiO}_{2}$ applications, similar results have started to reveal themselves in the near past [76-79], emphasizing the significance of anatase crystalline shape. The natural crystal form of this phase is a truncated tertragonal bipyramid having dominating facets ( $~ 94 \%$ of the overall surface area, [38]) with $\{101\}$ orientation that are capped by $\{001\}$ planes (Figure 21). If the structure expands perpendicular to $\{001\}$ surfaces, $\{100\}$ facets start to evolve on the sides forming a prismatic shape, eventually. Other crystalline structures - like distorted octahedrons in the third raw of Figure 21 containing high energy facets $(\{10 l\} ; l>1)$ are difficult to synthesize [78] and rarely reported to offer outstanding activity [224]. 


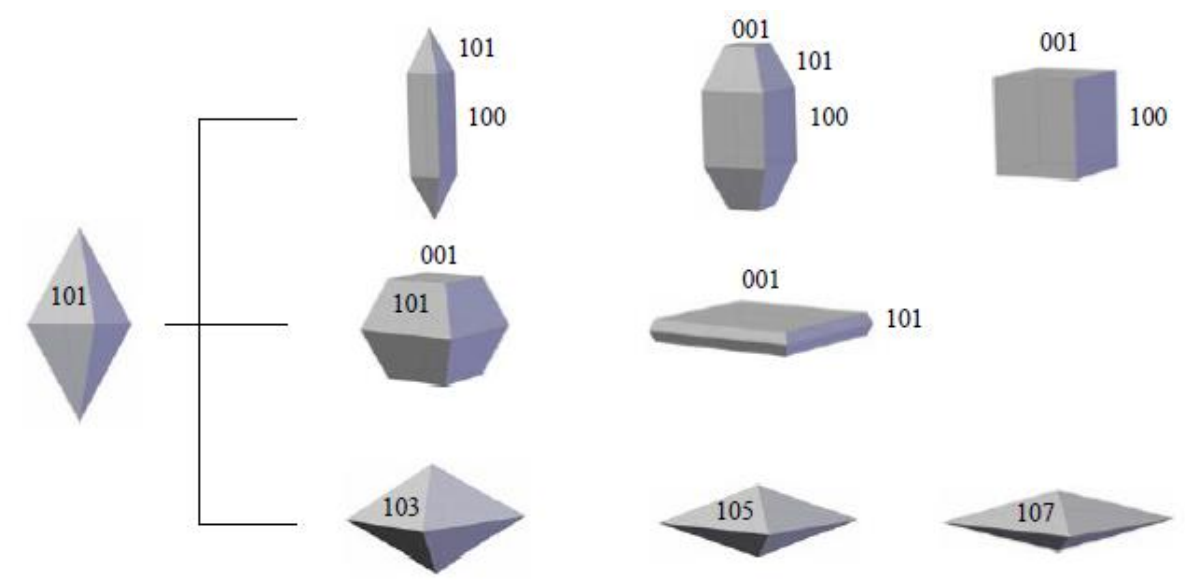

Figure 21: Anatase crystalline shapes with different facet ratios. Miller indices of each individual planes are indicated (Taken from [225]).

Out of $\{001\},\{101\}$ and $\{100\},\{001\}$ has the highest surface energy exposing only 5-fold coordinated $\mathrm{Ti}$ and a specifically big amount of 2-fold coordinated $\mathrm{O}_{\mathrm{Br}}$ atoms in a very strained configuration [78]. For instance, the Ti-O-Ti bond angle is very large which leads to an increased reactivity of these segments [226]. Owing to this advantageous atomic arrangement, an exceptionally high density of - predominantly terminal type - hydroxyl groups is obtained on \{001\} facets [227]. Furthermore, a sort of synergetic effect was evidenced between $\{001\}$ and $\{101\}$ planes that originates from their local energy band structures [228]. The surface heterojunction formed at their joint boundaries guides photogenerated holes and electrons preferentially towards the former and the latter surfaces, respectively. It means, on the one hand, a more efficient spatial charge separation that works against recombination. Secondly, the fortunate combination of higher OH-group density and the increased availability of holes at the $\{001\}$ facets results in a significantly enhanced charge trapping and subsequent OH-radical production [79,229]. However, maximizing the $\{001\} /\{101\}$ surface ratio does not always bring the desired performance improvement, as in some cases, an optimum value could be detected for certain applications [230]. Like it was explicated in the literature - and being in accordance with our observations -, there are a plenty of extrinsic factors in such complex heterogeneous reaction systems (medium, reactants, etc.) that collectively define the global photocatalytic activity, by which the efficiency is assessed. Confining the treatment to catalyst quality, we encounter further complications: when tailoring particle morphology, we may uncontrollably configure e.g., the defect structure at the same time [78], that also influences some essential processes.

Anyhow, the above $\{001\} /\{101\}$ ratio-dependent efficiency enhancement is substantiated and provides the following conclusion to our case. The morphological classification we applied is not the most adequate one (albeit it seemed to work out that time), because even if there is a bigger difference between two samples' spherical particle counts, the activity-shape correlation can easily be distorted by the uncovered majority of $\{001\}$ facets. Unfortunately, direct quantitative information on facet 
distributions cannot be acquired by the analytical techniques we used. Reflections of $\{001\}$ do not appear on X-ray diffractograms in the $2 \Theta^{\circ}$ range recorded. Furthermore, it is impossible to identify all crystalline facets, to measure them and to create reliable statistics from TEM projections, since particles are oriented diversely on the image plane revealing merely their contours. It turned out only after we had completed our experimental investigations, that Raman spectroscopy would have been the most appropriate tool for ascertaining precise facet ratios [231]. We still had, however, two measurement methods in hand, potentially capable of providing us with indirect information on this characteristic through the knowledge of $\mathrm{OH}$-group densities; X-ray photoelectron spectroscopy was utilized for gathering data on surface chemical composition, while dynamic light scattering helped us in determining the extent of surface hydrophilicity and $\mathrm{pH}$-dependent hydrodynamic behavior of the particles. All these results will be presented later at the respective sections.

As mentioned earlier, a few A-samples were subjected to gas phase photocatalytic experiments as well to get a better insight into the working mechanisms of our catalysts. On the other hand, it was simply interesting to see what sort of differences will appear compared to liquid phase phenol decomposition. The biggest influence was predicted to be the medium itself since this is what essentially determines, for instance, diffusion processes, the chemical state of the $\mathrm{TiO}_{2}$ surface and of all species being involved in the reactions. Secondly, the adsorption and degradation mechanisms of the two gaseous components (described in Section 2.5.2) suggest a very different behavior from phenol's, which is rather a hydrophobic compound and breaks down in a more complicated manner. That is, acetic and formic acid (primary products of the gas phase reactions) appear only after phenol has already transformed through $\mathrm{OH}$-insertion, ring opening and multiple decarboxylation steps. Despite these differences, methanol decomposition experiments - unlike acetaldehyde's - brought nearly the same order of initial reaction rates as obtained for phenol (Table 8). This equally means that values of $r_{0 \text {,methanol }}$ also show a positive correlation with the frequency of polyhedral particles, while a somewhat opposite tendency, or almost no connection is found for acetaldehyde (Figure 22).

\begin{tabular}{|c|c|c|c|c|}
\hline $\begin{array}{c}\text { Sample ID } \\
\text { (burner- } \mathrm{H}_{2}: \mathrm{O}_{2}- \\
\text { VTiCl4- } \mathrm{T}_{\mathrm{TiCl}} \text { ) }\end{array}$ & $\begin{array}{l}\text { Activity/ro,phenol } \\
\qquad\left(\times 10^{-8} \mathrm{Ms}^{-1}\right)\end{array}$ & $\begin{array}{l}\text { Activity/ro,methanol } \\
\left(\times 10^{-8} \mathrm{Ms}^{-1}\right)\end{array}$ & $\begin{array}{l}\text { Activity/ro,acetaldehyde } \\
\qquad\left(\times 10^{-8} \mathrm{Ms}^{-1}\right)\end{array}$ & $\begin{array}{c}\text { Frequency of } \\
\text { polyhedral } \\
\text { particles }(\%)\end{array}$ \\
\hline A-0.96-118-30 & 12.80 & 1.15 & 3.41 & 48 \\
\hline A-0.96-178-30 & 11.30 & 0.84 & 2.63 & 33 \\
\hline A-0.96-237-30 & 15.90 & 1.87 & 3.05 & 59 \\
\hline A-0.96-237-30/2 & 15.60 & 1.90 & 2.62 & 49 \\
\hline A-0.96-355-30 & 20.20 & 2.18 & 2.30 & 69 \\
\hline P25-B & 15.40 & 1.76 & 2.80 & 100 \\
\hline
\end{tabular}

Table 8: Liquid as well as gas phase photocatalytic activities of P25-B and of A-samples investigated herein. 


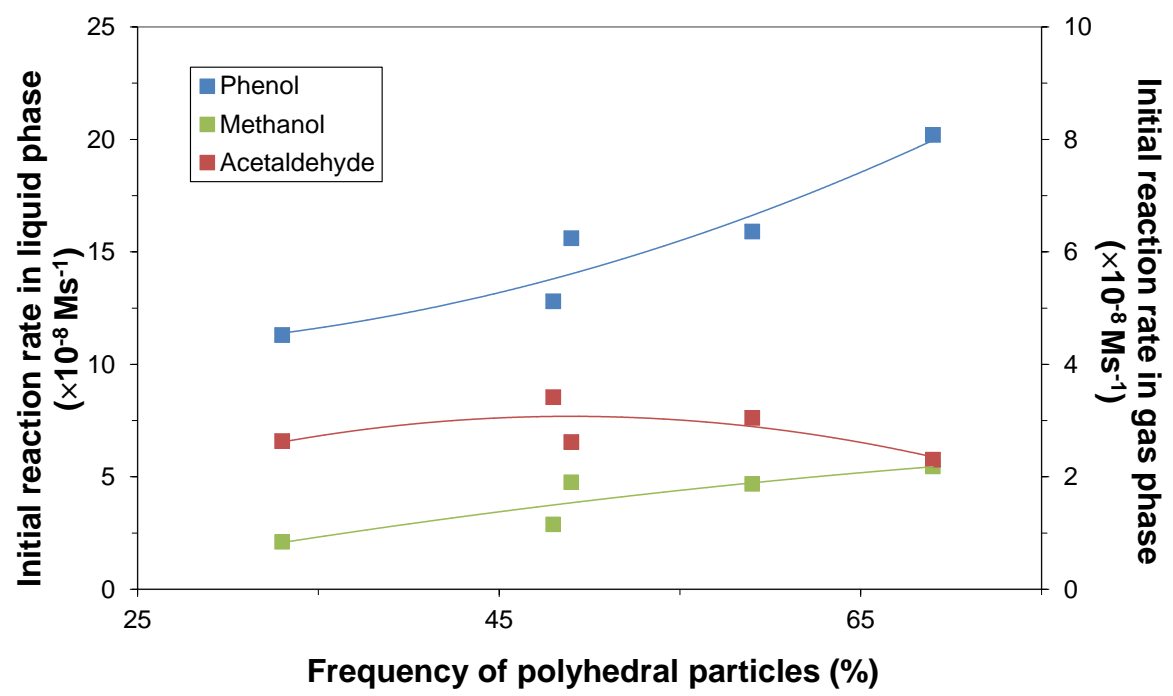

Figure 22: Photocatalytic activities obtained for both reaction media are plotted against the ratio of polyhedral particles. P25-B is intentionally omitted due to its bigger SSA and incomparable synthesis procedure.

In order to reveal the underlying reasons for these interrelations, results and mechanisms of all three model reactions will individually be examined in the following paragraphs.

Under our experimental conditions, there is no significant phenol adsorption, so the majority of these molecules will be transformed by radicals. From this fact, two conclusions can be drawn on phenol decomposition:

- Its adsorption kinetics do not play a decisive role.

- Up to a certain conversion range, the transformation rate is determined by the amount of radicals colliding with phenol molecules. This assumption is valid strictly for those reaction regimes where phenol molecules still appreciably outnumber radicals. When the situation turns over, the reaction rate will be then governed by the continuously decreasing concentration of the target compound.

An important relating deduction is that even if almost all adsorption/photocatalytically active sites of the $\mathrm{TiO}_{2}$ particles are occupied by intermediates, radicals shall still be liberated into the bulk medium without reacting any species but phenol itself. Using the following simple calculation, the theoretical possibility of reaching - at least nearly - full catalyst coverage by the intermediates during the decomposition runs can be proven. Taking the $\mathrm{TiO}_{2}$-loading $(100 \mathrm{mg})$, an average SSA $\left(25 \mathrm{~m}^{2} / \mathrm{g}\right)$, PC's average maximum concentration (approximately $3 \times 10^{-5} \mathrm{M}$ ) and its polar surface area of $c a .0 .4$ $\mathrm{nm}^{2}$ (regarded as an acceptable estimate on PC's effective "joining surface area" at the adsorption; [232]) will give a coverage already of $28 \%$. However, there are a few factors that could significantly increase this number:

- Simultaneous adsorption of HQ (less preferred than PC's) and other undetected aliphatic compounds; HQ's concentration maxima are usually about 2-times of PC's. It shall not be 
forgotten that these values are liquid phase equilibrium concentrations, so, it is very probable that particle surfaces are already being covered by these species at the given point time. This is even more valid for short-chain oxygenated aliphatic intermediates, whose overall concentration - based on the carbon balance - shall be higher than that of aromatics, if not consumed much faster.

- $\mathrm{O}_{2}$-adsorption; It can impede the adsorption of many oxygen containing substrates.

- Water adsorption; Water is known to be an efficient competitor even against oxygen containing small hydrocarbons (e.g., methanol and acetaldehyde) and is therefore - aside from $\mathrm{O}_{2}$ - the most plausible source of radicals under high coverage circumstances. In addition, owing to the aqueous medium, water is available in a quasi-infinite amount around catalyst particles and thus its adsorption is the least likely mass transport limited.

- SSA-reduction due to a certain extent of $\mathrm{TiO}_{2}$-aggregation

- The overall active surface area, that binds polluting molecules before chemical transformations, is lower than the SSA determined by $\mathrm{N}_{2}$-adsorption. Unlike model substances and their decomposition products, $\mathrm{N}_{2}$ adsorbs weakly and non-selectively to all regions of $\mathrm{TiO}_{2}$ surfaces this way providing accurate value of the real SSA.

Considering all these information, it is rationally assumed that $\mathrm{TiO}_{2}$ particles are initially almost "clean" - having, certainly, water, oxygen and perhaps some phenol molecules anchored -, while they will get more and more covered by intermediates on the way to total mineralization. But again, water and oxygen shall always occupy a given portion of catalytically active surface sites enabling persistent radical production.

To better aid the analysis of decomposition results, not only the decay curves of phenol but their negative time-derivatives are plotted in Figure 23 (right graph) as well, thereby visualizing reaction rate changes. Although these diagrams might look a bit crowded as data of all samples are simultaneously depicted, we found this type of representation beneficial in identifying common trends and differences in samples' behavior. 

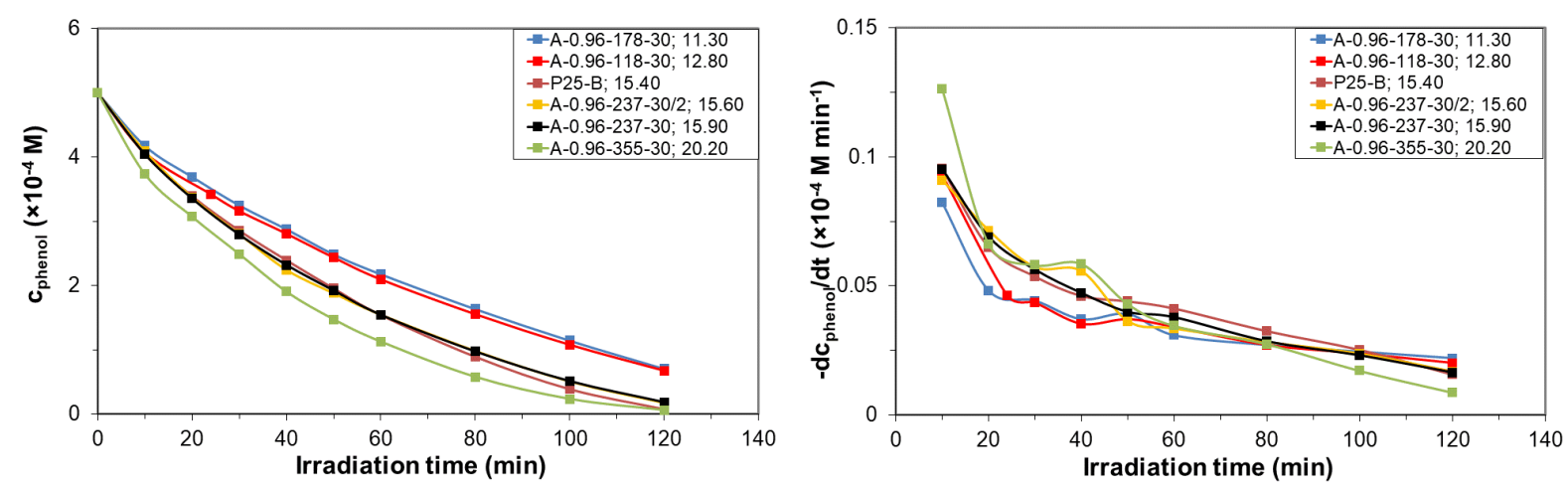

Figure 23: Phenol decomposition curves (left) and their negative time-derivatives (right). Insets contain photocatalytic activities $\left(\times 10^{-8} \mathrm{Ms}^{-1}\right)$, each separated by a semicolon from the respective sample code.

In the first phase of the degradation (approximately until the twentieth minute), a remarkable rate drop occurs at all investigated samples mainly due to the interfacial accumulation of intermediates that starts right away when switching on the UV-source ([73] and our TOC data in Chapter 4.3.8.). From this point on, reaction rates show quite different patterns for the individual $\mathrm{TiO}_{2} \mathrm{~s}$ up until $c a$. the $60^{\text {th }}$ minute: sometimes it simply continues to decrease with a smaller slope (P25-B), becomes constant for a certain period (A-0.96-237-30/2) or even passes through local maxima and minima (A-0.96-178-30). To be able to decipher these observed fine structures of the rate curves, precise information on both aqueous and adsorbed amounts of all substances shall be available. However, the manifold aliphatic compounds appearing in the liquid phase are hard to routinely separate and measure without applying special analytical techniques - in our relatively wide literature survey, no work was found with such results. Secondly, it seems even more problematic to carry out concentration profiling on $\mathrm{TiO}_{2}$ particles themselves. Although no such quantification is in hand, some may suspect that reaction systems exhibiting rate fluctuations in this regime are balancing at a pseudo-zero-order kinetics - limited by the rate of radical production -, that is continuously perturbed by the partially mass transport-controlled adsorption and degradation kinetics of the intermediates. As mentioned before, $\mathrm{pH}$ changes may further complicate the picture through altering the protonation state of reactants and of the $\mathrm{TiO}_{2}$ surface. Without in situ $\mathrm{pH}$ data any speculation is pointless, but one thing can confidently be stated: colloidal stability of our systems is most likely preserved throughout the reactions due to the low $\mathrm{pH}_{\mathrm{IEP}}$ values of the samples (except $\mathrm{P} 25-\mathrm{B}$ ), as discussed in Section 5.7. Howsoever, after the second stage comes the final one (from $c a$. the sixtieth minute) within which, characteristically again to all samples, phenol's decomposition rate decelerates on a nearly constant level up to the end of the experimental run, most probably indicating the dominant role of the gradually decreasing low phenol concentrations.

Besides the fundamental differences between aqueous and gas phase reaction circumstances, there is another major one: it can be demonstrated by the aid of the calculation method presented 
before, that gas phase degradations start from a relatively high substrate-catalyst ratio. The overall initial polar surface area of the model compounds is a 300-400-times higher than the available $\mathrm{TiO}_{2}$ surface that is still to be further reduced to obtain the active surface represented by the adsorption centers themselves. Certainly, co-adsorption of water and oxygen must be taken into account here as well, their number exceeds the target molecules' by three-four orders of magnitude. It directly follows that less than $0.2 \%$ of the substrates could be bound to particles, which fact gives explanation on why no measurable adsorption was detected before starting the illumination of the gas phase reaction systems. This low surface coverage, however, does not mean that the contribution of the holetriggered oxidation route to the overall decomposition is negligible. Assuming that a given substrate breaks down through the same elementary steps - or rather, through the same intermediates - at both routes, the radical-assisted mechanism shall be somewhat slower. This originates partially from the lower statistical probability of successful collisions between radicals and gas phase target molecules (versus an ideal, fully hole-mediated reaction), since only a certain portion of photogenerated holes will transform into radicals for which, in addition, other species are competing as well. Furthermore, the aggregate time constant of the event chain radical production $\rightarrow$ release $\rightarrow$ diffusion in medium is definitely bigger than for hole oxidation. We believe that this interrelation is reflected by our experimental data and, thus, the determining role of initial acetaldehyde adsorption is confirmed. Namely, despite methanol's $12 \%$ higher initial concentration and its fewer mineralization reaction steps, significantly higher $\mathrm{r}_{0}$-s were measured for acetaldehyde under the same circumstances (Table 8). Literature findings (Section 2.5) also support the conclusion, that even though a relative humidity of around 50-60\% is supposed to exist in our system, acetaldehyde might not fully be replaced by water within the first stage of the decomposition. By contrast, methanol was reported to be completely displaced from the $\mathrm{TiO}_{2}$ surface already at a one order of magnitude lower water-to-methanol ratio than what was applied in our investigations.

In Figures 24 and 25, similarly to phenol's case, both decay and rate curves are shown for all $\mathrm{TiO}_{2} \mathrm{~S}$ subjected to gas phase tests, from which the latter type will again serve as a basis for the upcoming dissection. 

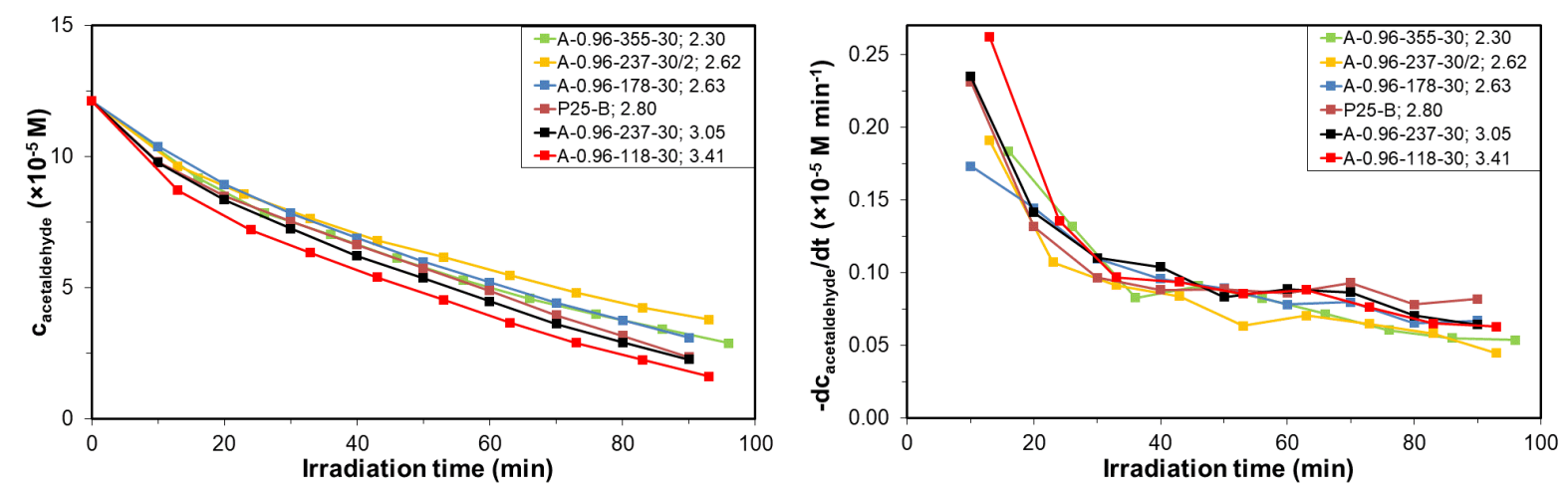

Figure 24: Acetaldehyde decomposition curves (left) and their negative time-derivatives (right). Insets contain photocatalytic activities $\left(\times 10^{-8} \mathrm{Ms}^{-1}\right)$, each separated by a semicolon from the respective sample code.

Acetaldehyde decomposition reaction rates vary almost identically for each sample (Figure 24). At the beginning, curves exhibit a steep drop because of the gradual accumulation of intermediates. Then, passing through a sometimes quite distinct breakpoint located roughly between the $20^{\text {th }}$ and the $40^{\text {th }}$ minutes, they continue decreasing with a smaller slope or stay almost constant until the end of the reaction. Even though the direct hole oxidation mechanism is decisive for the overall breakdown rate, it is hard to tell in which timeframe it becomes insignificant compared to radical-triggered routes. Perhaps right around the breakpoints, where acetaldehyde conversions reach some 40-50\%, but it might equally be sustained on a certain level for longer reaction times. Within the last stage - in contrast to phenol -, reaction rate decrease is presumably not driven by the diminishing substrate concentration, since the remaining amount of acetaldehyde is estimated to be at least one order of magnitude higher than the number of photocatalytically active $\mathrm{TiO}_{2}$ sites. This characteristic, for instance, can be an indication of a longer preserved but gradually decreasing extent of acetaldehyde adsorption. Let us pick the case of P25-B, whose SSA is twice as big as our samples'. It is obviously able to bind more acetaldehyde longer, therefore, it shows the highest degradation rate at the end of the reactions (beyond the $70^{\text {th }}$ minute).
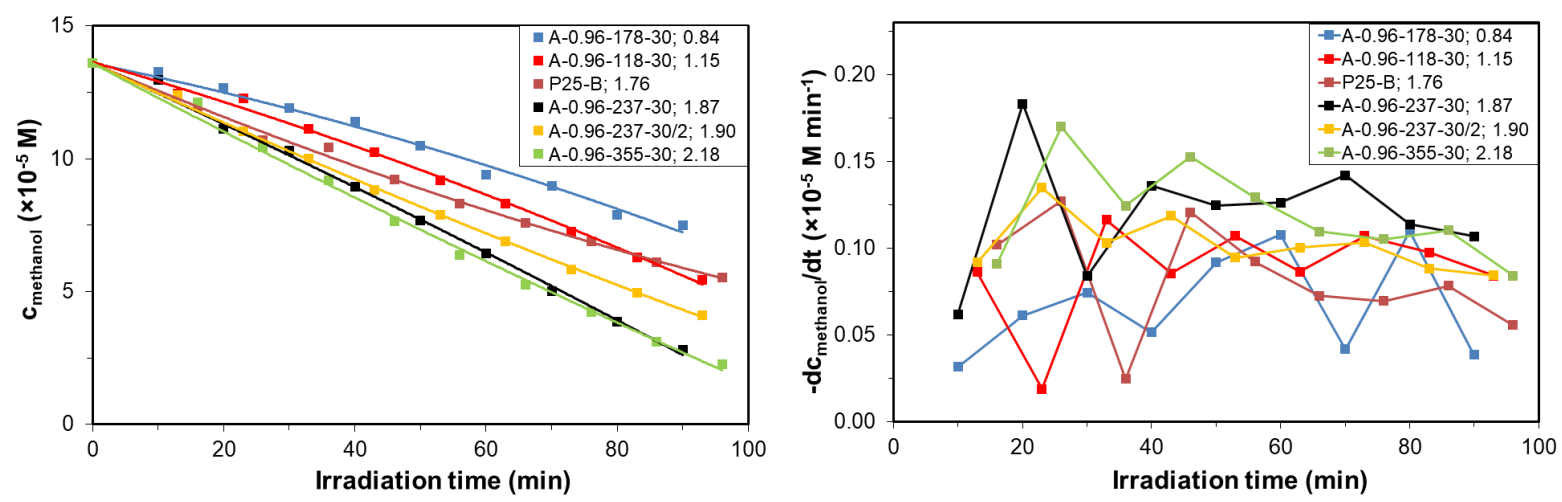

Figure 25: Methanol decomposition curves (left) and their negative time-derivatives (right). Insets contain photocatalytic activities $\left(\times 10^{-8} \mathrm{Ms}^{-1}\right)$, each separated by a semicolon from the respective sample code. 
As for methanol degradation, we obtained quite confusing rate curves (Figure 25, right graph). At first glance, one might think that such behavior must originate from some kind of measurement error. However, temporal variations of the graphs are too uniform: they all show very high extent of fluctuation at the beginning which gradually settles down towards the end of the run (except at sample A-0.96-178-30). Moreover, some of them have nearly identical shapes (P25-B and A-0.96-355-30) with data points slightly shifted along the ordinate. These features, we believe, rule out the possibility of having either random or systematic error incorporated into the results. Unfortunately, there is no publication found where similar experimental circumstances were applied and reaction rates were calculated to the entire timescale, so it is not possible to unravel potential root causes of the present observations. One fact seems to be indisputable, after all: methanol molecules are fully displaced from the $\mathrm{TiO}_{2}$ surface before initiating the reaction. Additionally, the situation most likely stays unchanged for the whole time of the degradation due to the accumulation of the well-adsorbing intermediates and to the remaining, still excessive amount of water. Consequently, there is no other way for methanol but to be decomposed by free radicals, as it was declared for phenol. This similarity must be the link between these two model reactions and it leads us to postulate that polyhedral particles have an appreciably higher efficiency in producing free radicals. Only this explains why the initial reaction rate increases for weakly adsorbing target compounds while it worsens or stays nearly constant for acetaldehyde when having more and more polyhedral grains in the samples.

Finally, there are some facts to draw the attention to. Despite it has an approximately twofold bigger SSA and it consists exclusively of polyhedral grains, P25-B's performance was always overtaken by a few of our samples. As we declared before, bigger SSA can bring fundamental advantage in decomposing well-adsorbing substances, like acetaldehyde. On the other hand, if it increases beyond a threshold, quantum efficiencies might drop by generating more lattice defects serving as recombination centers [67-68]. Another aspect we have previously explored is the ratio of specific facets constituting particle surfaces. Even if our catalysts contain less polyhedral particles, they might expose a larger aggregate area of $\{101\}$ planes, overcompensating disadvantageous impacts of other properties. Concerning particle morphology in general, samples with more polyhedral grains exhibited higher $\mathrm{r}_{0}$ values for the poorly adsorbing methanol and phenol, whereas this feature could not really be beneficial in acetaldehyde degradation reactions. So, it became obvious that, though, each individual structural parameter can alone be determining, collective influence of all sample characteristics configures the overall photocatalytic activity, eventually. 


\subsection{Burner-B synthesis runs}

It was already explained in the previous chapter that those technical problems appeared at Burner-A made it necessary to fabricate Burner-B. Its structure was carefully designed in a way to eliminate all the weaknesses of the former flame reactor. Also an important goal was to obtain more data points within wider synthesis parameter ranges in order to identify the subtle changes of catalyst properties and to better map the ideal operational conditions of the burner. The experiments started with the $\mathrm{H}_{2}-\mathrm{O}_{2}$ ratio adjustment. This time, the overall gas flow rate was kept more or less constant $(8446 \pm 125 \mathrm{~mL} / \mathrm{min})$ which meant an unchanged particle residence time throughout the entire series. This fact can be quantitatively verified by checking linear gas velocity ratios of the precursor feed and the outer air flow (Table 9). While it has changed from 2.5 to 4.1 for Burner-A, here a much smaller increase was achieved as it was ranging between 7.1 and 7.9. This slight variation as well as the higher value itself makes it reasonable to assume that, unlike at Burner-A, the precursor flow is not disturbed significantly during the synthesis. Another indirect evidence for this may be the monotonously changing catalyst properties (Figure 27 and 28).

\begin{tabular}{|c|c|c|c|c|c|c|}
\hline $\begin{array}{c}\text { Sample ID } \\
\text { (burner-H2: } \mathrm{O}_{2-} \\
\text { VTiCl4- }_{\mathrm{TiCl}} \text { ) }\end{array}$ & $\begin{array}{l}\mathrm{H}_{2} \text { flow } \\
\text { rate } \\
(\mathrm{mL} / \mathrm{min})\end{array}$ & $\begin{array}{c}\text { Precursor flow } \\
\text { rate } \\
\text { (mL/min) }\end{array}$ & $\begin{array}{c}\text { Outer air flow } \\
\text { rate } \\
\text { (mL/min) }\end{array}$ & $\begin{array}{c}\mathrm{H}_{2} \text { velocity } \\
(\mathrm{m} / \mathrm{min})\end{array}$ & $\begin{array}{l}\text { Precursor } \\
\text { velocity } \\
(\mathrm{m} / \mathrm{min})\end{array}$ & $\begin{array}{l}\text { Outer air } \\
\text { velocity } \\
(\mathrm{m} / \mathrm{min})\end{array}$ \\
\hline B-1.00-355-30 & 1422 & 355 & 6743 & 56.6 & 12.6 & 1.8 \\
\hline B-1.11-355-30 & 1577 & 355 & 6743 & 62.8 & 12.6 & 1.8 \\
\hline B-1.18-355-30 & 1629 & 355 & 6574 & 64.8 & 12.6 & 1.7 \\
\hline B-1.21-355-30 & 1642 & 355 & 6405 & 65.4 & 12.6 & 1.7 \\
\hline B-1.24-355-30 & 1681 & 355 & 6405 & 66.9 & 12.6 & 1.7 \\
\hline B-1.24-355-30/2 & 1681 & 355 & 6405 & 66.9 & 12.6 & 1.7 \\
\hline B-1.28-355-30 & 1732 & 355 & 6405 & 68.9 & 12.6 & 1.7 \\
\hline B-1.34-355-30 & 1771 & 355 & 6236 & 70.5 & 12.6 & 1.6 \\
\hline B-1.34-355-30/2 & 1771 & 355 & 6236 & 70.5 & 12.6 & 1.6 \\
\hline B-1.38-355-30 & 1771 & 355 & 6067 & 70.5 & 12.6 & 1.6 \\
\hline B-1.41-355-30 & 1810 & 355 & 6067 & 72.1 & 12.6 & 1.6 \\
\hline
\end{tabular}

Table 9: Gas flow rates used for the synthesis of B-samples produced by varying the $\mathrm{H}_{2}-\mathrm{O}_{2}$ molar ratio.

Heading towards higher $\mathrm{H}_{2}-\mathrm{O}_{2}$ ratios, gradual drop of anatase content and simultaneous increase of anatase particle size can be detected. This behavior is fully consistent with the general principle according to which a bigger amount of $\mathrm{H}_{2}$ present in the flame will necessarily result in a higher flame temperature (up to the stoichiometric $\mathrm{H}_{2}-\mathrm{O}_{2}$ ratio). Hence, assuming unchanged flame geometry and particle trajectory, the temperature can be increased to such an extent that not only anatase particles will grow but rutile grains also start getting larger when anatase size goes beyond the thermodynamic threshold of phase transition. As it was presumed, the degree of dispersity was increasing with increasing flame temperature (Figure 26 and Table 10). 

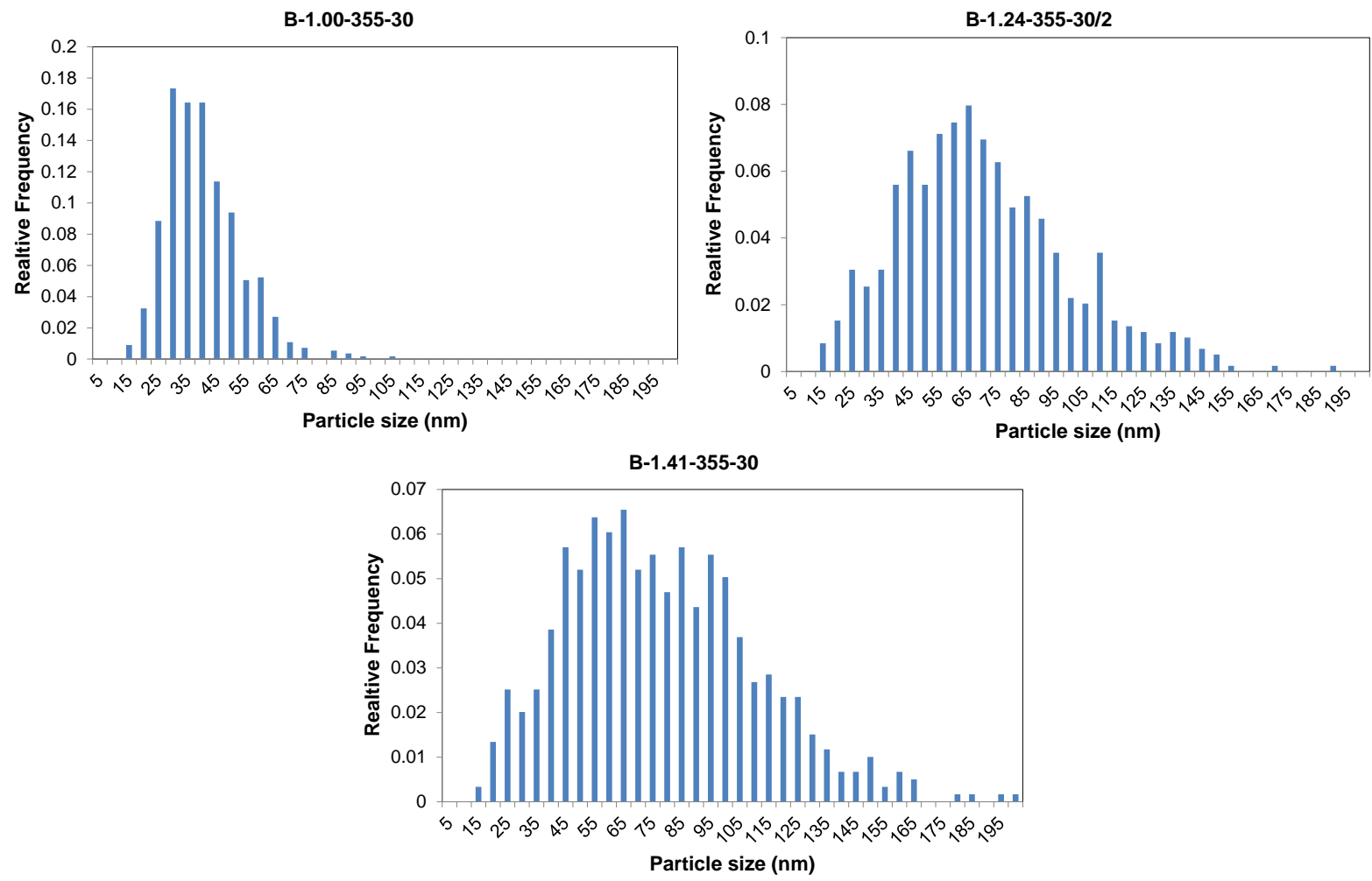

Figure 26: TEM particle size distribution histograms of B-samples produced by varying the $\mathrm{H}_{2}-\mathrm{O}_{2}$ molar ratio.

It is seen in Figure 28 that rutile sizes are almost always above those of anatase particles. This observation definitely fits the theory that anatase is formed first and transforms into rutile only when the cluster size exceeds a certain value. There are only two exceptions visible where rutile particles were smaller than anatase grains.

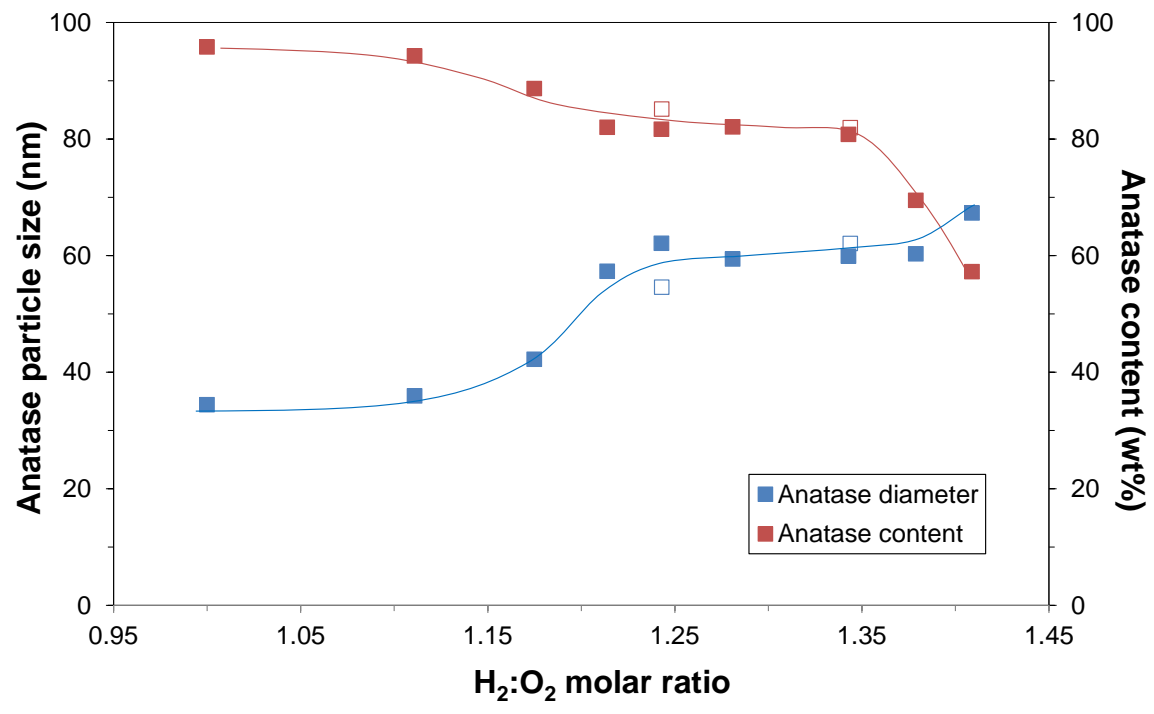

Figure 27: $\mathrm{H}_{2}-\mathrm{O}_{2}$ molar ratio dependency of anatase content and anatase particle size at Burner-B. Empty symbols represent samples originating from synthesis reproductions. 


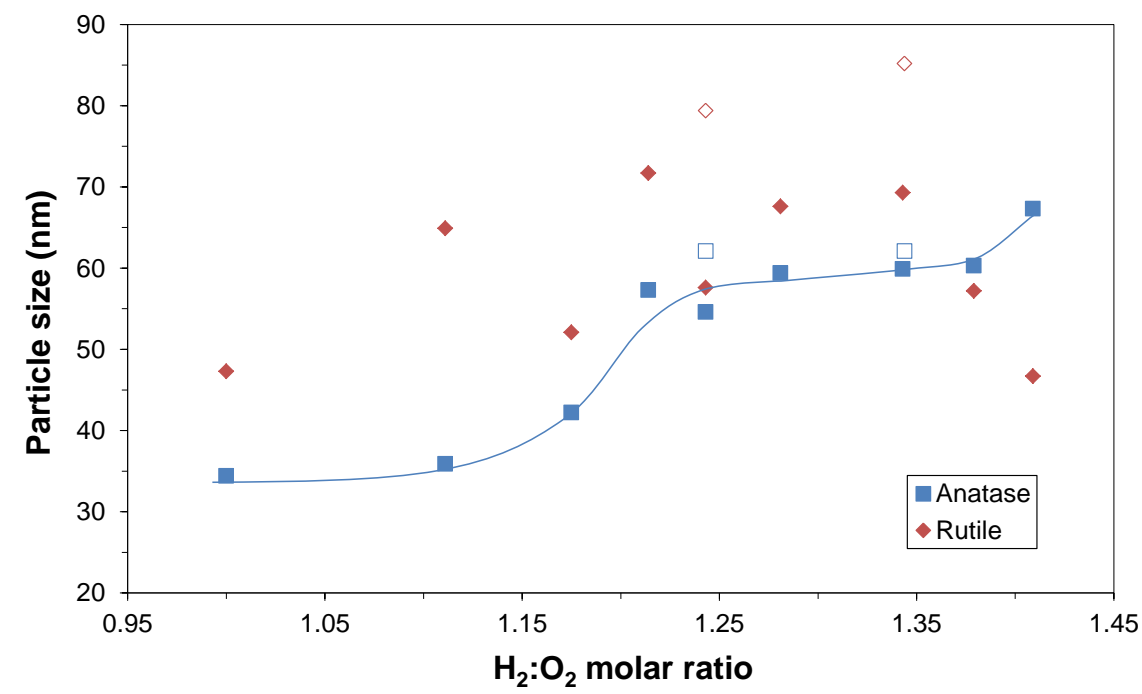

Figure 28: $\mathrm{H}_{2}-\mathrm{O}_{2}$ molar ratio dependency of both anatase and rutile particle sizes at Burner-A. Empty symbols represent samples originating from synthesis reproductions.

An interpretation is offered by Zhao et al. [233] who declared that the increased quantity of anatase oxygen vacancies could promote phase transformation by opening the way for a lower activation energy lattice reconstruction. Those two particular samples mentioned have been produced with the highest $\mathrm{H}_{2}-\mathrm{O}_{2}$ molar ratios which - besides providing higher flame temperatures - created an $\mathrm{O}_{2}$-lean environment in the reaction zone. The quasi-reductive circumstances finally led to the formation of oxygen-deficient crystal structures having a bigger quantity of $\mathrm{Ti}^{3+}$ centers incorporated, whose presence was easily perceptible by the blueish color of samples concerned. As a consequence of the oxygen-deficient state, rutile starts to evolve from a given portion of anatase clusters even at the early stages of particle formation while the rest of the material - having more oxygen in the lattice - stays stabilized in anatase form. It directly follows that the higher the hydrogen concentration is, the smaller the transforming anatase particles are, which eventually results in smaller rutile particles. Nevertheless, in comparison with the respective A-samples, material properties exhibit much larger and more systematic variation here (Table 10), partially due to the correct $\mathrm{H}_{2}-\mathrm{O}_{2}$ ratio regulation and to the extended experimental range. 


\begin{tabular}{|c|c|c|c|c|c|}
\hline $\begin{array}{c}\text { Sample ID } \\
\text { (burner- } \mathrm{H}_{2}: \mathrm{O}_{2-} \\
\left.\text { VTiCl4- }_{\text {TiCl4 }}\right)\end{array}$ & $\begin{array}{c}\text { Anatase } \\
\text { content } \\
(w t \%)\end{array}$ & $\begin{array}{l}\text { Anatase size } \\
\text { / XRD }(\mathrm{nm})\end{array}$ & $\begin{array}{l}\text { Rutile size / } \\
\text { XRD (nm) }\end{array}$ & $\begin{array}{l}\text { Particle size } \\
\text { / TEM (nm) }\end{array}$ & $\begin{array}{l}\text { Degree of } \\
\text { dispersity } \\
\text { / TEM (nm) }\end{array}$ \\
\hline B-1.00-355-30 & 96 & 34 & 47 & 38 & 22 \\
\hline B-1.11-355-30 & 94 & 36 & 65 & - & - \\
\hline B-1.18-355-30 & 89 & 42 & 52 & - & - \\
\hline B-1.21-355-30 & 82 & 57 & 72 & - & - \\
\hline B-1.24-355-30 & 85 & 55 & 58 & - & - \\
\hline B-1.24-355-30/2 & 82 & 62 & 79 & 69 & 56 \\
\hline B-1.28-355-30 & 82 & 59 & 68 & - & - \\
\hline B-1.34-355-30 & 82 & 62 & 85 & - & - \\
\hline B-1.34-355-30/2 & 81 & 60 & 69 & - & - \\
\hline B-1.38-355-30 & 69 & 60 & 57 & - & - \\
\hline B-1.41-355-30 & 57 & 67 & 47 & 76 & 70 \\
\hline
\end{tabular}

Table 10: Structural parameters of $\mathrm{B}$-samples produced by varying the $\mathrm{H}_{2}-\mathrm{O}_{2}$ molar ratio.

Burner-B syntheses were continued with precursor feed rate change series. Former conclusions on burner design-specific particle formation principles drawn from A-sample characteristics were successfully justified by the actual experimental results. As it appears in the corresponding figures (29 and 30), raising the precursor flow rate from 178 to $237 \mathrm{~mL} / \mathrm{min}$ results in anatase particle size increase and in practically unchanged phase composition. As discussed earlier, such kind of "irregular" behavior may originate from the relatively constant (at least not decreasing) precursor gas phase concentration and the simultaneous flame temperature increase, which latter is caused by the increased amount of $\mathrm{TiCl}_{4}$ transforming into titania within a unit time and a unit volume.

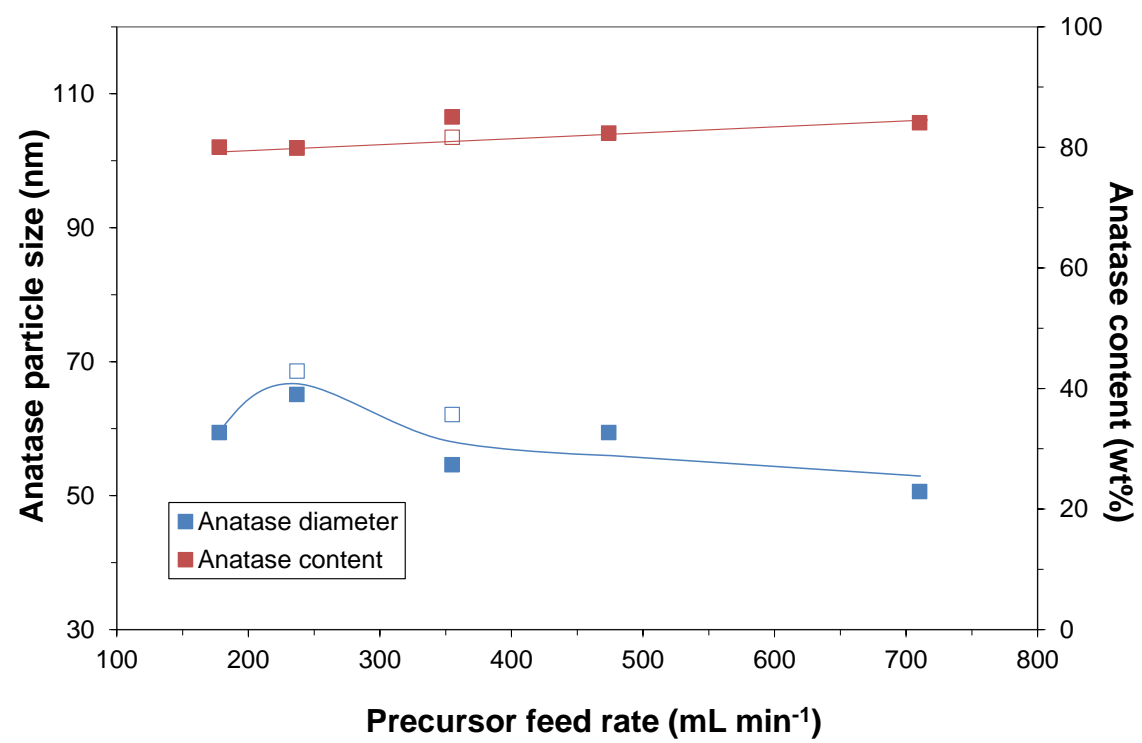

Figure 29: $\mathrm{TiCl}_{4}$ feed rate dependency of anatase content and anatase particle size at Burner-B. Empty symbols represent samples originating from synthesis reproductions. 


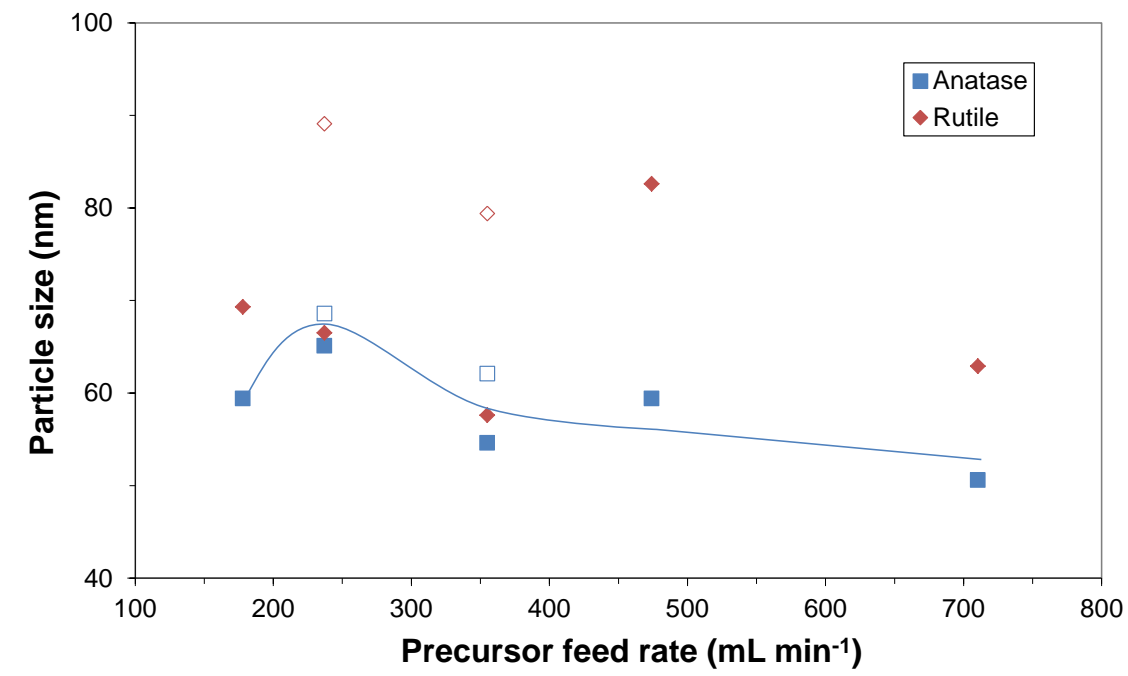

Figure 30: $\mathrm{TiCl}_{4}$ feed rate dependency of both anatase and rutile particle sizes at Burner-B. Empty symbols represent samples originating from synthesis reproductions.

\begin{tabular}{|c|c|c|c|c|c|c|}
\hline $\begin{array}{c}\text { Sample ID } \\
\text { (burner-H } \\
\text { v }_{\text {TiCl4 }}-\mathrm{T}_{\mathrm{TiCl}} \text { ) }\end{array}$ & $\begin{array}{c}\mathbf{H}_{2} \text { flow rate } \\
(\mathrm{mL} / \mathrm{min})\end{array}$ & $\begin{array}{l}\text { Precursor } \\
\text { flow rate } \\
\text { (mL/min) }\end{array}$ & $\begin{array}{l}\text { Outer air flow } \\
\text { rate } \\
\text { (mL/min) }\end{array}$ & $\begin{array}{c}\mathbf{H}_{2} \text { velocity } \\
(\mathrm{m} / \mathrm{min})\end{array}$ & $\begin{array}{l}\text { Precursor } \\
\text { velocity } \\
\text { ( } \mathrm{m} / \mathrm{min})\end{array}$ & $\begin{array}{l}\text { Outer air } \\
\text { velocity } \\
(\mathrm{m} / \mathrm{min})\end{array}$ \\
\hline B-1.24-178-30 & 1681 & 178 & 6582 & 66.9 & 6.3 & 1.7 \\
\hline B-1.24-237-30 & 1681 & 237 & 6523 & 66.9 & 8.4 & 1.7 \\
\hline B-1.24-237-30/2 & 1681 & 237 & 6523 & 66.9 & 8.4 & 1.7 \\
\hline B-1.24-355-30 & 1681 & 355 & 6405 & 66.9 & 12.6 & 1.7 \\
\hline B-1.24-355-30/2 & 1681 & 355 & 6405 & 66.9 & 12.6 & 1.7 \\
\hline B-1.24-474-30 & 1681 & 474 & 6286 & 66.9 & 16.8 & 1.7 \\
\hline B-1.24-710-30 & 1681 & 710 & 6050 & 66.9 & 25.1 & 1.6 \\
\hline
\end{tabular}

Table 11: Gas flow rates used for the synthesis of B-samples produced by varying the precursor feed.

When passing further to higher feed rates, both sample properties start showing a different tendency than before: anatase content increases while particle diameters start decreasing. This occurs due to the gradual precursor dilution as evaporation cannot provide enough molecules anymore to maintain a more or less constant gas phase concentration with continuously increasing volumetric flow. Just like at Burner-A, particle size distribution data provides another appropriate proof for the above theory here as well. In Figure 31, all three graphs are shown that were derived from TEM data of the present series of catalysts (Table 12). Since sample B-1.24-355-30/2 is located close to the turn-point, its average particle size is still bigger than that of B-1.24-178-30, nevertheless, the degree of dispersity definitely decreases with increasing $\mathrm{TiCl}_{4}$ feed rate. 

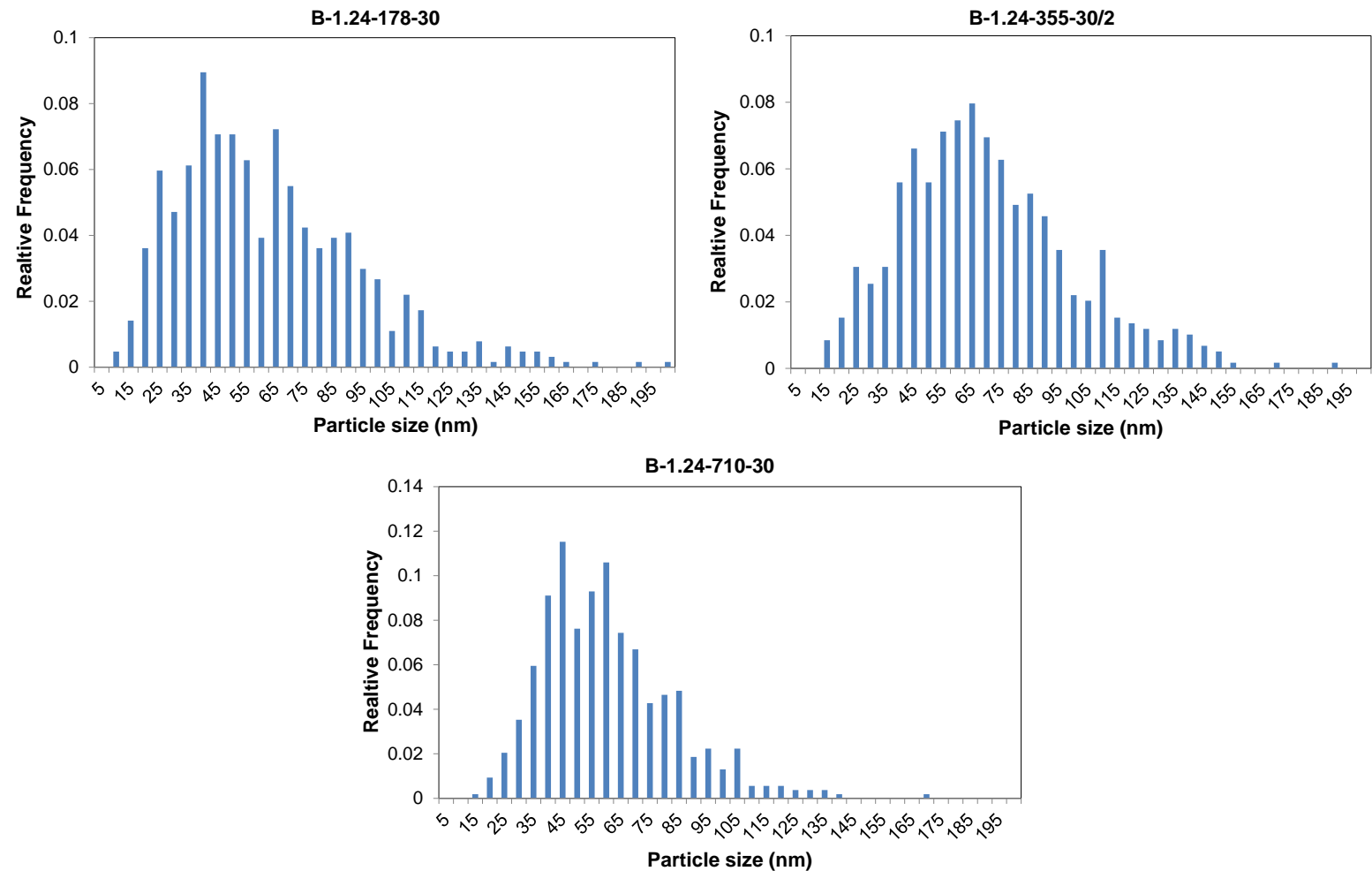

Figure 31: TEM particle size distribution histograms of B-samples produced by varying the precursor feed rate. The degree of dispersity is decreasing with increasing precursor feed rate.

Certainly, there are some differences in synthesis parameter ranges and in material properties between precursor feed rate experiments of the two flame reactors that are induced mostly by burner geometries. The most representative example is the turn-point (of the precursor gas phase concentration) itself which is situated at somewhat lower feed rates than in the case of Burner-A. However, identical characteristics with regard to the evolution of phase composition and of particle size have ultimately verified the correctness of all flame synthesis principles that we have established.

\begin{tabular}{|c|c|c|c|c|c|}
\hline $\begin{array}{c}\text { Sample ID } \\
\text { (burner-H2: }{ }_{2}- \\
\text { VTiCl4- } \mathrm{T}_{\mathrm{TiCl}} \text { ) }\end{array}$ & $\begin{array}{l}\text { Anatase } \\
\text { content } \\
(w t \%)\end{array}$ & $\begin{array}{l}\text { Anatase size } \\
\text { / XRD (nm) }\end{array}$ & $\begin{array}{l}\text { Rutile size / } \\
\text { XRD (nm) }\end{array}$ & $\begin{array}{l}\text { Particle size } \\
\text { / TEM (nm) }\end{array}$ & $\begin{array}{l}\text { Degree of } \\
\text { dispersity } \\
\text { / TEM (nm) }\end{array}$ \\
\hline B-1.24-178-30 & 80 & 59 & 69 & 60 & 66 \\
\hline B-1.24-237-30 & 80 & 65 & 67 & - & - \\
\hline B-1.24-237-30/2 & 80 & 69 & 89 & - & - \\
\hline B-1.24-355-30 & 85 & 55 & 58 & - & - \\
\hline B-1.24-355-30/2 & 82 & 62 & 79 & 69 & 56 \\
\hline B-1.24-474-30 & 82 & 59 & 83 & - & - \\
\hline B-1.24-710-30 & 84 & 51 & 63 & 59 & 46 \\
\hline
\end{tabular}

Table 12: Structural parameters of B-samples produced by varying the precursor feed rate.

As Burner-B showed persistently stable and reliable operation without encountering such technical problems like at Burner-A, the opportunity presented itself for testing the impacts of other synthesis parameters as well. At first, we wanted to see whether any of the sample characteristics will change by increasing the precursor gas phase concentration while all other parameters are kept 
constant. There was only one possibility to create such circumstances: to increase the thermostating water temperature that is flowing around the $\mathrm{TiCl}_{4}$-containing vial. For this purpose, we have chosen the synthesis parameters of sample B-1.24-355-30/2 as a baseline, since the highest photocatalytic activity was achieved there. The precursor temperature was ramped up to $50^{\circ} \mathrm{C}$ and to $70^{\circ} \mathrm{C}$ in the course of two individual runs. Having higher concentration of $\mathrm{TiCl}_{4}$ in the flame should basically be accompanied by enhanced molecular/particle collision rate and by higher flame temperature due to the increasing total reaction enthalpy. These conditions are then expected to induce the following variations in material properties: bigger grain sizes, lower anatase content and higher degree of dispersity. According to the negligible differences observed between B-1.24-355-30/2 and B-1.24355-70 structural characteristics (Figure 32, Table 13) one would assume practically unchanged synthesis circumstances. Nevertheless, catalyst quality was surely modified in some ways, because there is a remarkable $25 \%$ drop in photocatalytic activity when setting the precursor temperature to $70^{\circ} \mathrm{C}$. Surprisingly, polyhedral particle counts do not differ either, which recalls again what was explained with respect to crystalline facet ratios. As XPS measurements were conducted only on sample B-1.24-355-30/2 out of this set, we have insufficient information to figure out what characteristics have been changed here and how they depend on the current synthesis variable.

\begin{tabular}{|c|c|c|c|c|c|c|c|}
\hline $\begin{array}{c}\text { Sample ID } \\
\text { (burner-H } \\
\text { v }_{\text {TiCl4 }}-\mathrm{T}_{\mathrm{TiCl}} \text { ) }\end{array}$ & $\begin{array}{l}\text { Anatase } \\
\text { content } \\
(w t \%)\end{array}$ & $\begin{array}{c}\text { Anatase } \\
\text { size / XRD } \\
(\mathbf{n m})\end{array}$ & $\begin{array}{c}\text { Rutile size } \\
\text { / XRD } \\
\text { (nm) }\end{array}$ & $\begin{array}{l}\text { Particle } \\
\text { size } \\
\text { / TEM } \\
(\mathbf{n m})\end{array}$ & $\begin{array}{c}\text { Degree of } \\
\text { dispersity } \\
\text { / TEM (nm) }\end{array}$ & $\begin{array}{c}\text { Frequency of } \\
\text { polyhedral } \\
\text { particles } \\
(\%)\end{array}$ & $\begin{array}{c}\text { Activity/ } \\
\text { ro,phenol } \\
\left(\times 10^{-8}\right. \\
\left.\mathrm{Ms}^{-1}\right)\end{array}$ \\
\hline B-1.24-355-30/2 & 82 & 62 & 79 & 69 & 56 & 96 & 25.70 \\
\hline B-1.24-355-50 & 81 & 57 & 74 & - & - & & 24.74 \\
\hline B-1.24-355-70 & 81 & 63 & 73 & 71 & 56 & 97 & 19.35 \\
\hline
\end{tabular}

Table 13: Structural parameters and activities of B-samples produced by varying the liquid precursor temperature.
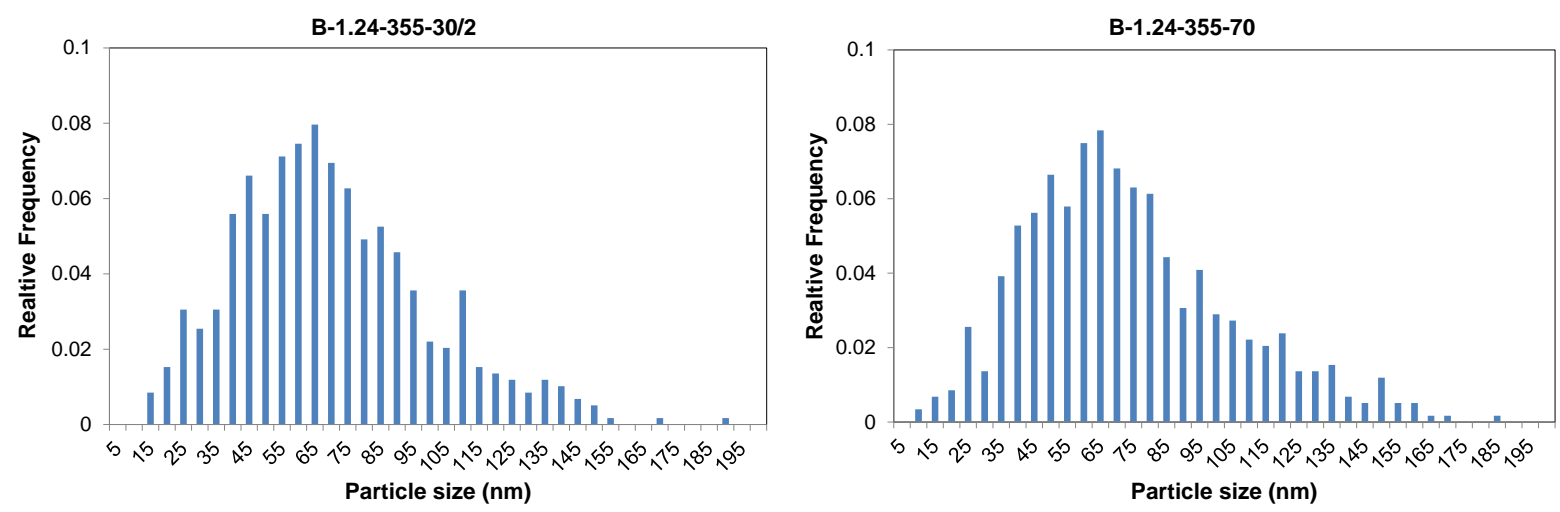

Figure 32: TEM particle size distribution histograms of B-samples produced by varying the liquid precursor temperature.

The remaining item in the set of potential synthesis parameter adjustments is the overall flow rate modification. This was achieved by simultaneously increasing/decreasing both the outer air and 
the hydrogen flow rates applied at sample B-1.24-355-30/2 again, while everything else was kept constant. That means, more specifically, unchanged precursor feed $(355 \mathrm{~mL} / \mathrm{min})$ and $\mathrm{H}_{2}-\mathrm{O}_{2}$ ratio (1.24). The extent of adjustment was calculated so to obtain a $20 \%$ lower and $20 \%$ higher overall flow rate than that set up for B-1.24-355-30/2. All synthesis parameters and corresponding sample codes are shown in Table 14.

\begin{tabular}{|c|c|c|c|c|c|c|}
\hline $\begin{array}{c}\text { Sample ID } \\
\text { (burner-H2: } \\
\text { vTiCl4- }_{\text {TiCl4) }}\end{array}$ & $\begin{array}{c}\mathrm{H}_{2} \text { flow } \\
\text { rate } \\
\text { (mL/min) }\end{array}$ & $\begin{array}{l}\text { Outer air } \\
\text { flow rate } \\
\text { (mL/min) }\end{array}$ & $\begin{array}{l}\text { Total air } \\
\text { flow rate } \\
\text { (mL/min) }\end{array}$ & $\begin{array}{l}\text { Precursor } \\
\text { velocity } \\
(\mathrm{m} / \mathrm{min})\end{array}$ & $\begin{array}{c}\mathrm{H}_{2} \\
\text { velocity } \\
\text { (m/min) }\end{array}$ & $\begin{array}{l}\text { Outer air } \\
\text { velocity } \\
(\mathrm{m} / \mathrm{min})\end{array}$ \\
\hline B-1.24-355-30-0.8 & 1344 & 5053 & 6752 & 12.6 & 53.5 & 1.3 \\
\hline B-1.24-355-30/2 & 1681 & 6405 & 8441 & 12.6 & 66.9 & 1.7 \\
\hline B-1.24-355-30-1.2 & 2017 & 7757 & 10129 & 12.6 & 80.3 & 2.0 \\
\hline
\end{tabular}

Table 14: Gas flow rates used for the synthesis of B-samples produced by varying the overall flow rate.

Basically, two direct effects are supposed to be evoked by varying the overall flow rate in the manner described. On the one hand, characteristics and the interaction of the individual gas streams might be altered. However, considering the large differences between their linear velocities (see the last three columns in Table 14), it is not very likely that even such an extent of change would be able to significantly modify the fluid-dynamical circumstances. The second and more obvious option is the flame temperature increase induced by the increasing overall flow rate, since bigger and bigger volumes of fuel is burnt per unit time. From this aspect, phase composition and rutile diameters vary accordingly, while anatase particle sizes fluctuate quasi randomly, but still within a relatively narrow range (Table 15).

\begin{tabular}{|c|c|c|c|c|c|c|}
\hline $\begin{array}{c}\text { Sample ID } \\
\text { (burner-H2:O2- } \\
\text { VTiCl4- }_{\text {TiCl4) }}\end{array}$ & $\begin{array}{l}\text { Anatase } \\
\text { content } \\
(w t \%)\end{array}$ & $\begin{array}{l}\text { Anatase } \\
\text { size / XRD } \\
(\mathbf{n m})\end{array}$ & $\begin{array}{l}\text { Rutile size } \\
\text { / XRD } \\
\text { (nm) }\end{array}$ & $\begin{array}{c}\text { Particle size } \\
\text { / TEM } \\
(\mathbf{n m})\end{array}$ & $\begin{array}{l}\text { Degree of } \\
\text { dispersity } \\
\text { / TEM (nm) }\end{array}$ & $\begin{array}{c}\text { Activity } \\
\text { /ro,phenol } \\
\left(\times 10^{-8} \mathrm{Ms}^{-1}\right)\end{array}$ \\
\hline B-1.24-355-30-0.8 & 85 & 55 & 71 & - & - & 20.82 \\
\hline B-1.24-355-30/2 & 82 & 62 & 79 & 69 & 56 & 25.70 \\
\hline B-1.24-355-30-1.2 & 82 & 55 & 83 & - & - & 20.87 \\
\hline
\end{tabular}

Table 15: Structural parameters and activities of B-samples produced by varying the overall flow rate.

The situation thus seems to be similar to that of the precursor temperature variation: small changes in material properties were accompanied by significant activity drop. Nevertheless, there is only one polyhedral particle frequency data available (for sample B-1.24-355-30/2, not indicated in Table 15), therefore, the grain morphology-activity relationship cannot either be dissected.

With respect to the foregoing two sets of experiments, it is hard to reliably verify our assumptions on particle formation mechanisms given for the individual cases, since there is insufficient number of data points at both runs. The reason is - apart from the technical limitations of 
parameter adjustment - that the main aim of our studies was to understand the correlation between structural properties and photoactivities, and to increase the latter as much as possible through synthesis optimization. When it turned out that any change of these two parameters leads to activity deterioration, we decided on discarding these routes.

\subsection{Photocatalytic activity of B-samples}

In the previous chapter, activities of some B-samples have already been presented along with structural characteristics and synthesis conditions in order to preserve the consistency of our treatment. The following evaluation is therefore fully devoted to the "core" $\mathrm{TiO}_{2} \mathrm{~s}$ prepared by precursor feed rate and $\mathrm{H}_{2}-\mathrm{O}_{2}$ molar ratio adjustment. For these, - just like for their Burner-A counterparts - a comprehensive correlation analysis could be implemented as an appropriate number of catalysts were produced to detect the basic tendencies of all features. However, unlike at Asamples, only aqueous phase phenol decomposition reaction was applied here, because: (i) gas phase reactions were thoroughly studied before and a detailed comparison with respect to adsorption and degradation mechanisms has already been provided; (ii) there was more potential seen in phenol reactions, since its results combined with the upcoming - and also aqueous phase - DLS and $\mathrm{O}_{2}$ consumption measurements were expected to deepen our insight into the possible set of activitydetermining factors. It is to be highlighted, that at B-samples, we time to time double-checked the reproducibility of photocatalytic phenol decomposition experiments. These second activity values are indicated in each relevant table (after slashes) and diagram, further on.

Photocatalytic activity shows a maximum at a $\mathrm{H}_{2}-\mathrm{O}_{2}$ ratio of 1.24 (Figure 33 and Table 16). Up to this point, samples' activities are gradually increasing while particle size and the rutile content keeps increasing as well, all along the entire $\mathrm{H}_{2}-\mathrm{O}_{2}$ ratio range investigated. Interestingly, the portion of polyhedral particles is always above (or equal to) $90 \%$ this time and exhibits only a $10 \%$ drop when heading from the lowest towards the highest $\mathrm{H}_{2}$ content of the flame. 


\begin{tabular}{|c|c|c|c|c|}
\hline $\begin{array}{c}\text { Sample ID } \\
\text { (burner-H2: } \\
\text { VTiCl4- } \mathrm{T}_{\mathrm{TiCl} 4} \text { ) }\end{array}$ & $\begin{array}{c}\text { Anatase } \\
\text { content } \\
(w t \%)\end{array}$ & $\begin{array}{l}\text { Anatase size } \\
\text { / XRD (nm) }\end{array}$ & $\begin{array}{l}\text { Activity/ro,phenol } \\
\left(\times 10^{-8} \mathrm{Ms}^{-1}\right)\end{array}$ & $\begin{array}{c}\text { Frequency of } \\
\text { polyhedral } \\
\text { particles }(\%)\end{array}$ \\
\hline B-1.00-355-30 & 96 & 34 & $15.47 / 15.68$ & 100 \\
\hline B-1.11-355-30 & 94 & 36 & 20.36 & - \\
\hline B-1.18-355-30 & 89 & 42 & $18.46 / 19.54$ & - \\
\hline B-1.21-355-30 & 82 & 57 & 22.57 & - \\
\hline B-1.24-355-30 & 85 & 55 & 24.19 & - \\
\hline B-1.24-355-30/2 & 82 & 62 & 25.70 & 96 \\
\hline B-1.28-355-30 & 82 & 59 & $24.34 / 24.10$ & - \\
\hline B-1.34-355-30 & 82 & 62 & 20.48 & - \\
\hline B-1.34-355-30/2 & 81 & 60 & 20.68 & - \\
\hline B-1.38-355-30 & 69 & 60 & 14.06 & - \\
\hline B-1.41-355-30 & 57 & 67 & 9.77 & 90 \\
\hline
\end{tabular}

Table 16: Structural parameters, photoactivities and shape statistics of B-samples produced by varying the $\mathrm{H}_{2}-\mathrm{O}_{2}$ molar ratio. Activities indicated after a slash represent results of independent photocatalytic degradation experiments.
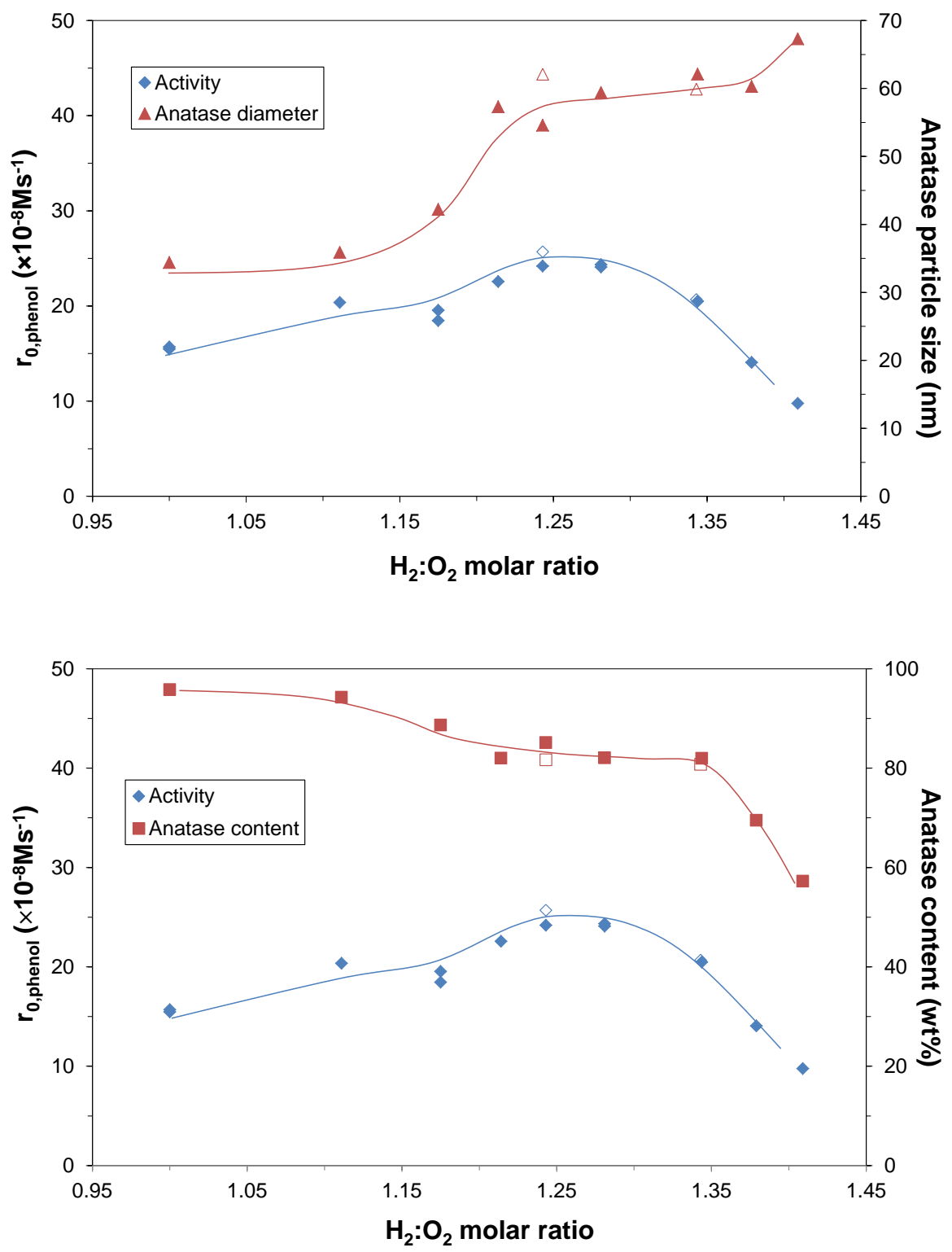

Figure 33: $\mathrm{H}_{2}-\mathrm{O}_{2}$ molar ratio dependency of anatase content, particle size and photocatalytic activity at Burner-B. Empty symbols represent samples originating from synthesis reproductions, while full symbols located at the same $\mathrm{H}_{2}-\mathrm{O}_{2}$ molar ratio indicate results of independent photocatalytic degradation experiments. 
Here, as already stressed, superimposed action of all material characteristics must be taken into account when analyzing the observable tendencies. If the presence of rutile lowers the overall photoactivity, then there must be some other properties, that can overcompensate the unfavorable modification of phase composition up to the $\mathrm{H}_{2}-\mathrm{O}_{2}$ ratio of $c a$. 1.24. One of these may be the particle size, which just reaches a plateau at exactly this gas composition. The theoretical background has been explained before: bigger grains are assumed to enable better spatial separation of charge carriers [54]. Furthermore, higher specific surface area is accompanied by having more lattice defects acting as recombination centers [67-68]. On the other hand, the impact of particle size increase turns over after exceeding a certain value, beyond which charge separation efficiency cannot further be improved but specific surface area starts playing a decisive role instead. Albeit the frequency of polyhedral particles is slightly decreasing with increasing $\mathrm{H}_{2}-\mathrm{O}_{2}$ ratio, grain morphology might still have a positive contribution up to the value of 1.24 , through the undetected prevalence of $\{101\}$ facets. Exactly the opposite influence is presumed to act within the interval of 1.28-1.34 (three samples included), where, in lack of other material property changes, the appearance of a more disadvantageous crystalline structure is most probably responsible for the activity drop. Then at the upmost range of the scale, the steep increase of the rutile content becomes dominant in configuring the remaining two samples' activities.

Precursor feed rate adjustment resulted in very small changes of all measurable sample characteristics (Figure 34 and Table 17). While anatase content and the number of polyhedral particles increase in a more or less monotonous fashion, particle size and photoactivity decrease, both reaching a peak value underway. The situation suggests that crystalline facet ratio may be even more decisive than at the previous series. However, any creditable statement on this interrelation can perhaps be made only after analyzing XPS data recorded deliberately for those three samples, whose morphologies were studied by TEM as well.

\begin{tabular}{|c|c|c|c|c|}
\hline $\begin{array}{c}\text { Sample ID } \\
\text { (burner-H } \\
\text { v }_{\text {TiCl4- }}: \mathrm{T}_{\mathrm{TiCl}} \text { ) }\end{array}$ & $\begin{array}{l}\text { Anatase } \\
\text { content } \\
(w t \%)\end{array}$ & $\begin{array}{l}\text { Anatase size / } \\
\text { XRD (nm) }\end{array}$ & $\begin{array}{l}\text { Activity/ro,phenol } \\
\left(\times 10^{-8} \mathrm{Ms}^{-1}\right)\end{array}$ & $\begin{array}{c}\text { Frequency of } \\
\text { polyhedral } \\
\text { particles (\%) }\end{array}$ \\
\hline B-1.24-178-30 & 80 & 59 & 21.42 & 95 \\
\hline B-1.24-237-30 & 80 & 65 & $19.41 / 19.63$ & - \\
\hline B-1.24-237-30/2 & 80 & 69 & 20.65 & - \\
\hline B-1.24-355-30 & 85 & 55 & 24.19 & - \\
\hline B-1.24-355-30/2 & 82 & 62 & 25.70 & 96 \\
\hline B-1.24-474-30 & 82 & 59 & $23.88 / 23.67$ & - \\
\hline B-1.24-710-30 & 84 & 51 & 20.39 & 97 \\
\hline
\end{tabular}

Table 17: Structural parameters, photoactivities and shape statistics of B-samples produced by varying the precursor feed rate. Activities indicated after a slash represent the results of degradation measurement repetitions. 

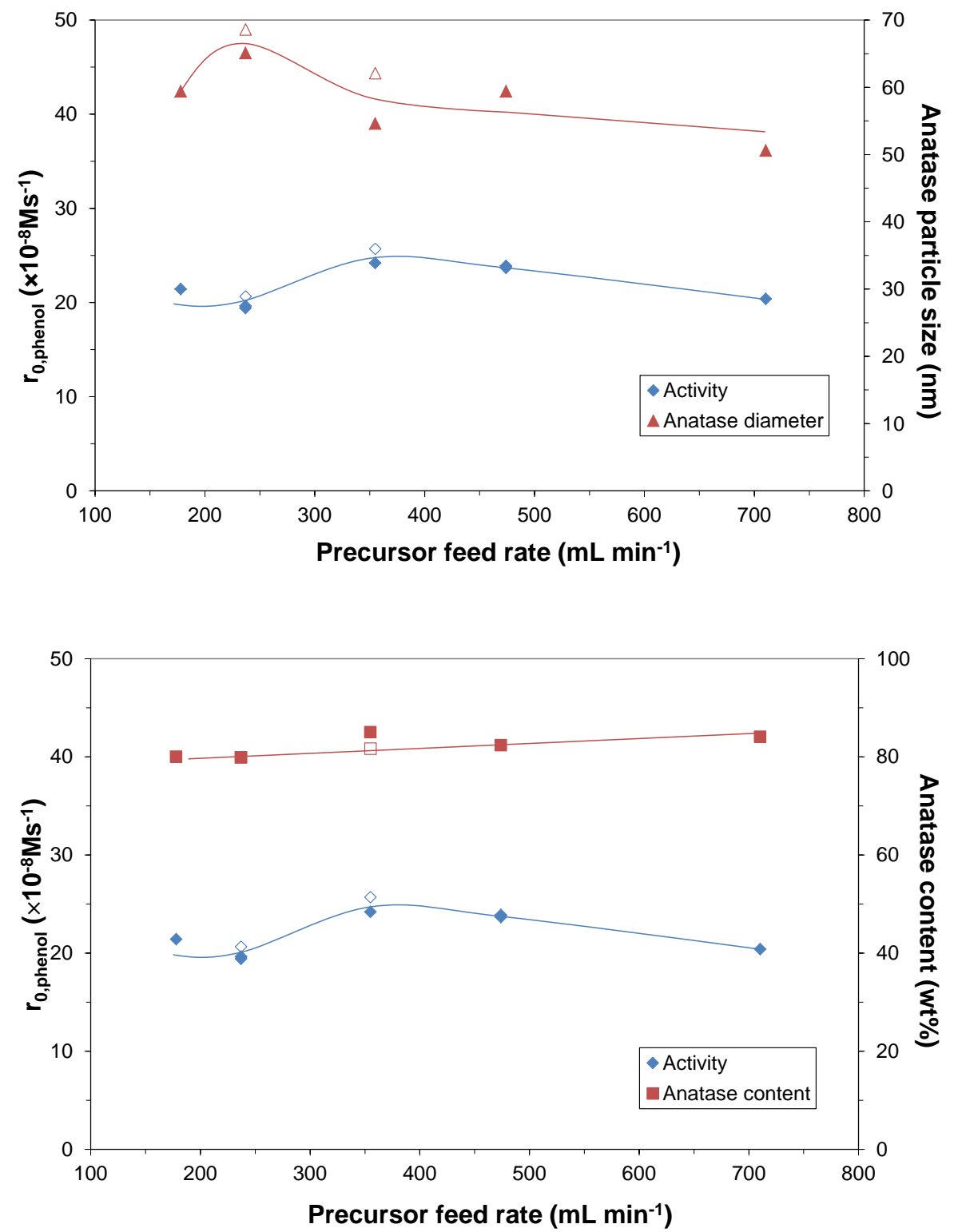

Figure 34: $\mathrm{TiCl}_{4}$ feed rate dependency of anatase content, particle size and photocatalytic activity at Burner-B. Empty symbols represent samples originating from synthesis reproductions while full symbols located at the same precursor feed rate value indicate results of independent photocatalytic degradation experiments.

Though the frequency of polyhedral grains has seemingly no direct impact on photocatalytic activities of B-samples, an important conclusion can be drawn from the data with respect to synthesis circumstances of such particles: it appears, that the formation of polyhedral grains favors lower flame temperatures and shorter reaction zone residence times. These tendencies are also observable at Burner-A series but must cautiously be treated. Like explained, an inadequate experimental design $\left(\mathrm{H}_{2}-\mathrm{O}_{2}\right.$ molar ratio regulation) and the unexpected variation of $\mathrm{TiCl}_{4}$ 's gas phase concentration created such synthesis conditions, where the opposing actions of precursor residence time and flame temperature could not suitably be isolated.

For summary, we plotted all B-sample and A-sample phenol degradation rates against particle size and phase composition just to provide a comprehensive visualized correlation analysis (Figure 
35). We do so, despite knowing that each material property must be taken into account at the same time when attempts are made to ascertain their relationship with photoactivities. Accordingly, individual representations may somewhat be misleading as they suggest the existence of some unrealistic trends. It is particularly valid to B-sample data, where - unlike at A-sample graphs - points are apparently arranged in a more regular pattern.
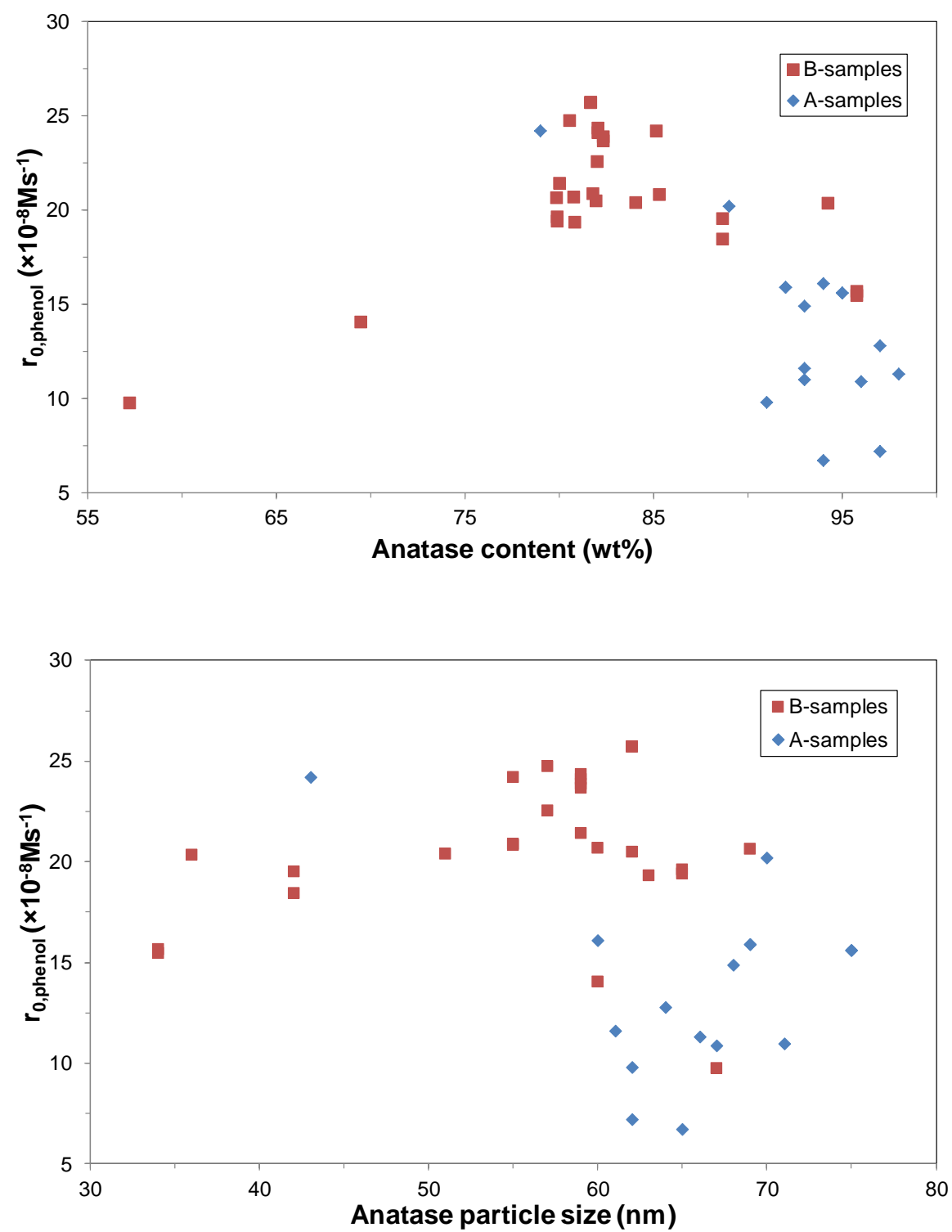

Figure 35: Photocatalytic activities of all A- and B-samples are shown as a function of anatase particle size and phase composition.

For instance, one could believe that there is a clear optimum of the anatase content at around $80 \%$, since samples situated close to this value have the highest phenol decomposition rate (Figure 35, upper graph). Nevertheless, the impression becomes weaker if we select and analyze the following two $\mathrm{TiO}_{2}$ S with the same phase composition: B-1.34-355-30 and B-1.24-355-30/2 (Table 16). Though all their other material properties are practically identical, they display an activity difference of $25 \%$. 
The scatter is even more pronounced when anatase particle diameter is set as variable (Figure 35, lower graph).

Present illustrations have another useful yield: they provide a rough quantitative notion on how much the data of the two sample series resemble each other. What shows up from the graphs right away is that B-samples, in general, possess higher photocatalytic activities. Additionally, their basic material properties are more diverse (i.e., extended to a wider range) than those of A-samples. There is only one exception to the previous rule. The frequency of polyhedral particles is always above or equal to $90 \%$ at B-samples, while it spreads along the full scale at A-samples. This fact is well-visible in Figure 36, which eventually describes the poor correlation between B-samples' particle morphologies and their photoactivities.

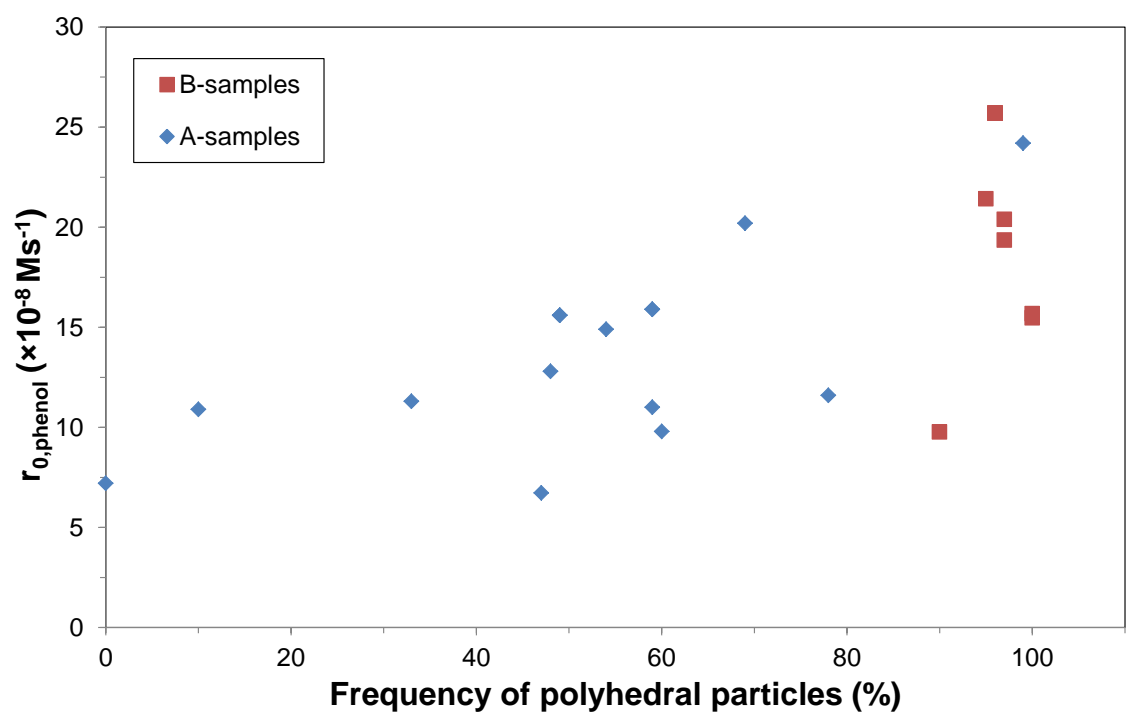

Figure 36: Initial phenol decomposition rates of A- and B-samples plotted as a function of polyhedral particle content.

We have seen so far that no general interrelation can be detected between $\mathrm{r}_{0}$-s and primary structural parameters. Those few exceptions found are worth highlighting, after all. Particle morphology has an unambiguous effect on activities of A-samples produced by precursor feed rate adjustment. Besides this, too low particle size and too high rutile content exert negative influence on phenol decomposition rate due to reasons already explained. It has got thus pointed out that, though, $\mathrm{TiO}_{2}$ characteristics determine activities collectively, any of them can become dominating over the others by reaching a given extremum. 


\subsection{XPS investigations}

Samples having nearly identical properties - including polyhedral particle count - have often displayed significant variations in their photocatalytic activities. Based on recent scientific findings, these differences might stem from the prevalence of $\{001\}$ oriented facets which, in turn, manifests itself in higher surface $\mathrm{OH}$-group density of primarily the terminal type [227]. Irrespective of the crystalline structure, researchers $[61,234]$ have already claimed earlier, that the amount of OH-groups is decisive in $\mathrm{TiO}_{2}$-mediated heterogeneous photocatalytic decomposition processes. For instance, Ardizzone et al. [234] pointed out that photocatalytic activity of nanocrystalline titania correlates with the density of surface $\mathrm{OH}$-groups: the best photocatalysts were found to be the ones showing the highest hydroxyl oxygen/oxide type (or lattice) oxygen surface ratio. Accordingly, we have also applied XPS with the expectation of finding any similar interrelation between surface chemical composition and photocatalytic activity. The availability of A-samples was unfortunately limited by their quantities because syntheses reproductions were not possible anymore due to Burner-A's breakdown. This is why we could pick only two of them for XPS measurements and that one of these has no XRD and TEM data. We did not have such a restraint with $\mathrm{B}$-samples, so three $\mathrm{TiO}_{2} \mathrm{~s}$ from the series of precursor flow rate alteration were chosen.

Apart from O-peaks, the analysis of the $\mathrm{Ti} 2 \mathrm{p}$ doublets can also have significance if a remarkable difference exists in samples' $\mathrm{Ti}^{4+} / \mathrm{Ti}^{3+}$ atomic ratio. Like already described, the presence of $\mathrm{Ti}^{3+}$ centers may influence photoactivity in several ways: they can act as charge trapping sites and can induce radical production. However, it turned out that any of the investigated samples contain no (or hardly detectable amount of) $\mathrm{Ti}^{3+}$. The contribution of this chemical state could be identified as a shoulder [235] located on the lower energy side of the Ti $2 \mathrm{p}_{3 / 2}$ peak (around $457.3 \mathrm{eV}$ ), which is actually not there at any of our $\mathrm{TiO}_{2}$ s (Figure 37). 


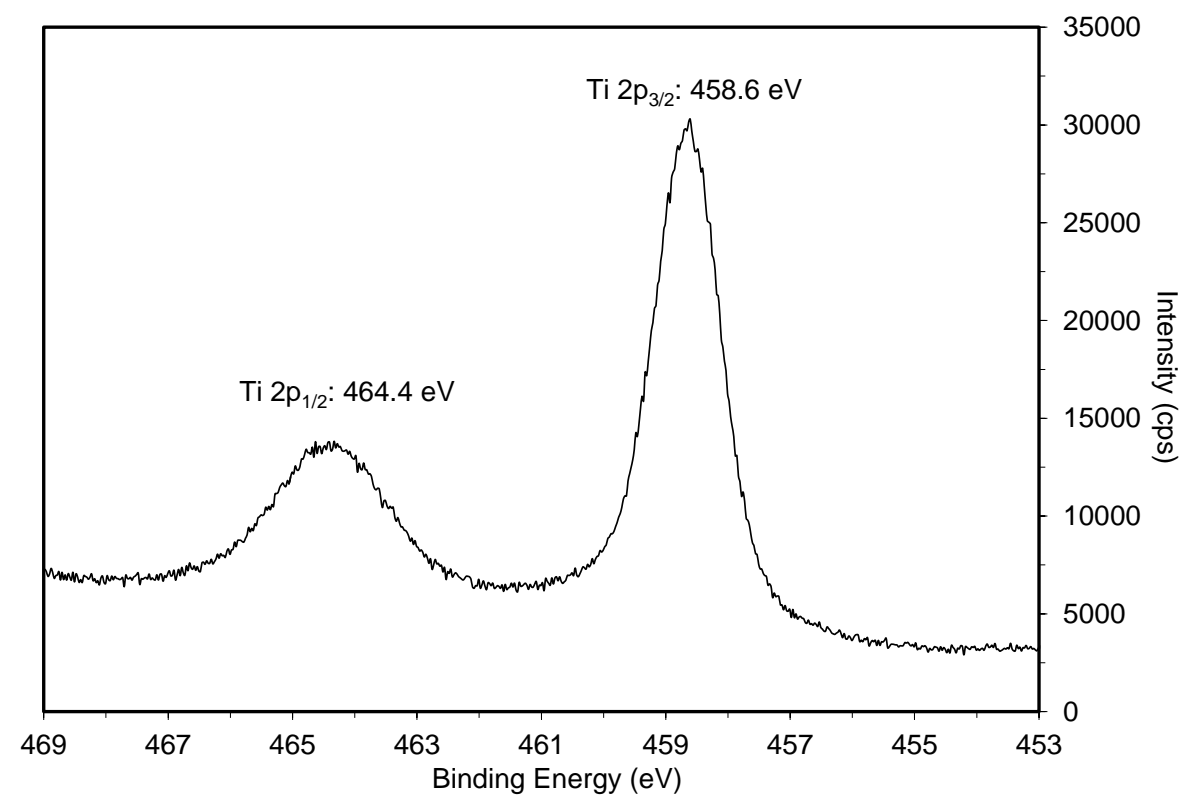

Figure 37: Representative Ti 2p spectrum of sample A-1.10-237-30.

The $\mathrm{O}$ 1s signal of clean $\mathrm{TiO}_{2}$ surfaces can typically be decomposed into three peaks: the most intense one located at $530.1 \mathrm{eV}$ belongs to lattice oxygen atoms (ID: O_oxide) while those around $531.6 \mathrm{eV}$ and $532.5 \mathrm{eV}$ are associated with surface bridging (ID: $O_{B r} H$ ) and terminal (ID: $O_{T} H$ ) $\mathrm{OH}-$ groups, respectively [235,236]. In Table 18, basic properties and $\mathrm{OH}-$ related atomic ratios of all measured samples are listed and their O 1s spectra are collected in Figure 38.
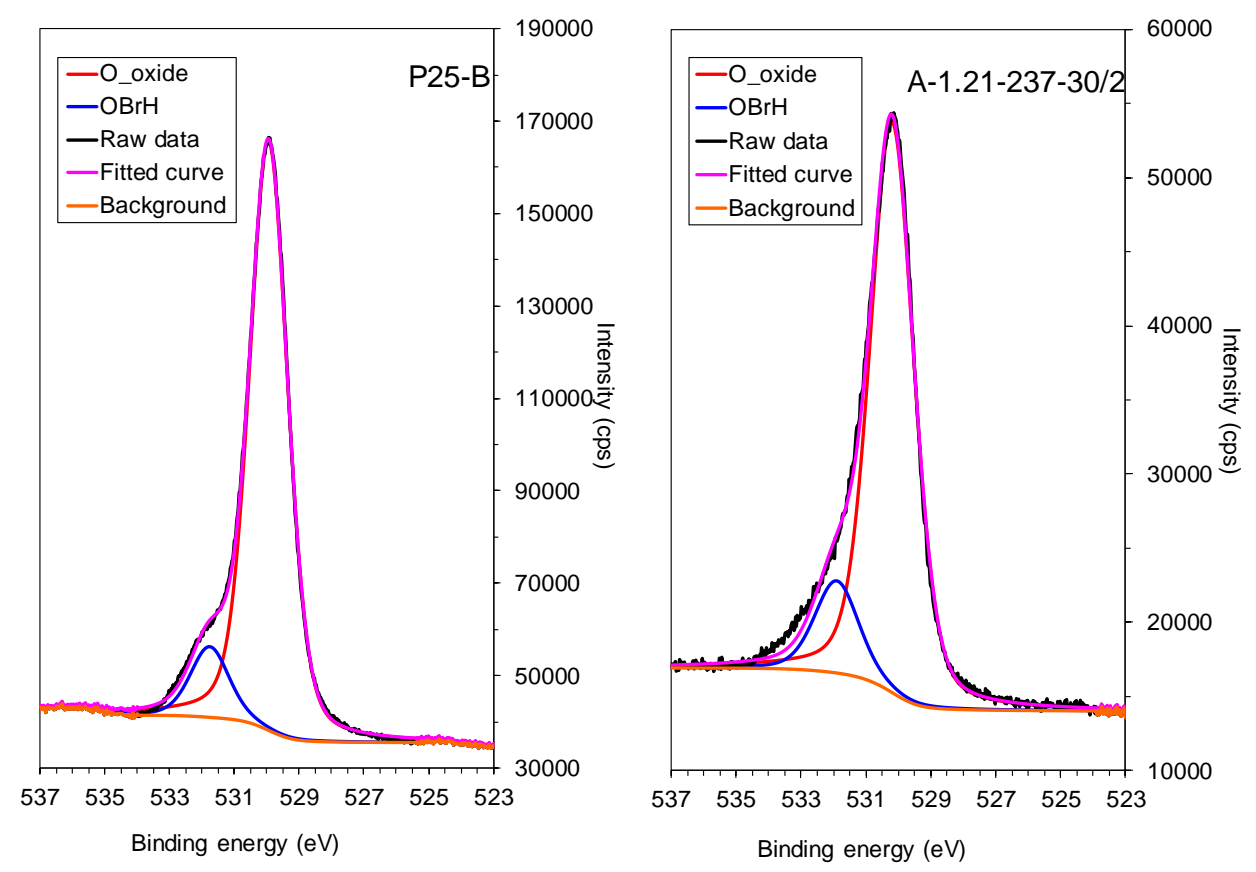

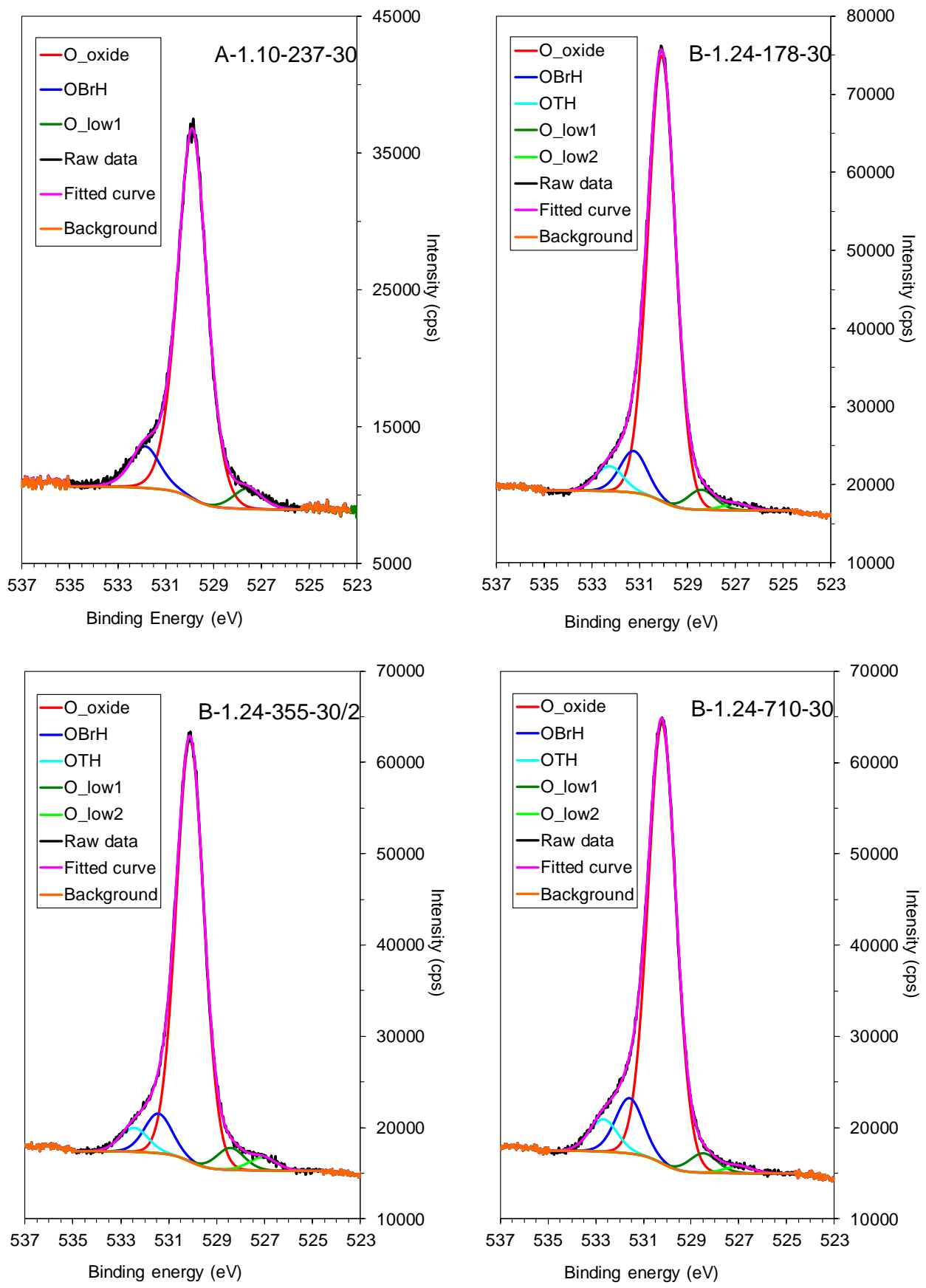

Figure 38: O 1s spectra of samples analyzed by XPS.

The $\mathrm{O}$ 1s signal of sample A-1.10-237-30 could be deconvoluted into the following three contributions. In addition to the oxide and the $\mathrm{O}_{\mathrm{Br}} \mathrm{H}-$ peak, there is a third, least intense component at $527.5 \mathrm{eV}$ (ID: $O \_l o w 1$ ). When O 1s signal analysis was continued with B-samples, five individual components had to be considered in order to obtain satisfactory fitting of the XPS curves. This means, that a further contribution appeared on the low (ID: O_low2) energy side of the lattice O-peak at $c a$. $527.0 \mathrm{eV}$. The origin of the two lowest energy peaks is unknown. Although the respective database and literature were thoroughly surveyed, no feature could be found with such a low $\mathrm{O} 1 \mathrm{~s}$ binding energy. These, hence, will not be involved in any further assessment. 


\begin{tabular}{|c|c|c|c|c|c|c|c|}
\hline \multirow{2}{*}{$\begin{array}{c}\text { Sample ID } \\
\text { (burner-H2: } \\
\text { viicl4- }^{\text {TiCl4 }} \text { ) }\end{array}$} & \multirow{2}{*}{$\begin{array}{c}\text { Anatase } \\
\text { content } \\
\text { (wt } \%)\end{array}$} & \multirow[t]{2}{*}{$\begin{array}{l}\text { Anatase size } \\
\text { / XRD (nm) }\end{array}$} & \multirow[t]{2}{*}{$\begin{array}{l}\text { Activity/ro,phenol } \\
\left(\times 10^{-8} \mathbf{M s}^{-1}\right)\end{array}$} & \multirow{2}{*}{$\begin{array}{c}\text { Frequency of } \\
\text { polyhedral } \\
\text { particles }(\%)\end{array}$} & \multicolumn{3}{|c|}{$\begin{array}{c}\text { Atomic ratios of surface hydroxyl } \\
\text { oxygen }\end{array}$} \\
\hline & & & & & $\begin{array}{c}\mathrm{O}_{\mathrm{Br} H} \\
(\mathbf{a} / \mathbf{a} \%)\end{array}$ & $\begin{array}{c}\mathbf{O}_{\mathrm{T}} \mathrm{H} \\
(\mathbf{a} / \mathbf{a} \%)\end{array}$ & $\begin{array}{c}\mathrm{O}_{\mathrm{Br}} \mathrm{H}+\mathrm{O}_{\mathrm{T}} \mathrm{H} \\
(\mathbf{a} / \mathbf{a} \%)\end{array}$ \\
\hline P25-B & 89 & 27 & 15.40 & 100 & 10.78 & 0 & 10.78 \\
\hline A-1.10-237-30 & - & - & 17.70 & - & 10.06 & 0 & 10.06 \\
\hline $\mathrm{A}-1.21-237-30 / 2$ & 97 & 62 & 7.20 & 0 & 13.94 & 0 & 13.94 \\
\hline B-1.24-178-30 & 80 & 59 & 21.42 & 95 & 8.13 & 4.87 & 13.01 \\
\hline B-1.24-355-30/2 & 82 & 62 & 25.70 & 96 & 8.15 & 4.94 & 13.09 \\
\hline B-1.24-710-30 & 84 & 51 & 20.39 & 97 & 10.48 & 6.11 & 16.60 \\
\hline
\end{tabular}

Table 18: Structural parameters, photoactivities and hydroxyl type oxygen $\left(\mathrm{O}_{\mathrm{Br}} \mathrm{H}, \mathrm{O}_{\mathrm{T}} \mathrm{H}\right)$ atomic ratios of samples investigated by XPS.

It has turned out at the very first step of the data analysis that the aggregate atomic ratio of the two types of hydroxyl oxygen atoms (Table 18, last column: $\mathrm{O}_{\mathrm{Br}} \mathrm{H}+\mathrm{O}_{\mathrm{T}} \mathrm{H}$ ) does not correlate to any of the other four properties examined. It is valid for $\mathrm{r}_{0}$ as well, which implies that it is insufficient to consider merely this cumulative quantity when forecasting activity ranks. Photophysical/chemical roles of $\mathrm{O}_{\mathrm{Br}} \mathrm{H}$ - and $\mathrm{O}_{\mathrm{T}} \mathrm{H}$-groups are still not fully mapped and generalizations are often applied in related publications (Sections 2.2-2.4). For instance, charge trapping function of these species is more or less verified (albeit some theories are debated) but specific contribution of the individual processes to the overall efficiency is unknown. Anyhow, distinct interrelations turn up if separately evaluating the impact of each kind of hydroxyls. Most importantly, the appearance of $\mathrm{O}_{\mathrm{T}} \mathrm{H}$-groups has unquestionably positive influence on B-samples' photoactivities. According to the literature, the increased amount of these species can be attributed to the enlarged exposure of $\{001\}$ facets which, in turn, opens the way for a more efficient charge separation process induced by the synergism between these and the $\{101\}$ facets [228]. It is very probable that this phenomenon is the most determining from photocatalytic activity point of view, nevertheless, substantive advantageous effect of $\mathrm{O}_{\mathrm{T}} \mathrm{H}$-groups cannot be ruled out either. It is more difficult to make firm statements on the origin and the role of $\mathrm{O}_{\mathrm{Br}} \mathrm{H}$-groups, because more structural properties are simultaneously changing within the set of investigated samples. Its quantity is seemingly growing with increasing anatase content (as declared for OH-groups, in general $[67,38]$ ) but the relatively low values measured for - at least two of the -B-samples may be a consequence of $\{101\}$ facet dominance. On the other hand, no connection can be revealed with particle size and polyhedral particle count. From these two aspects, it is worth comparing sample A-1.21-237-30/2 with P25-B. Latter consists exclusively of polyhedral grains and has more than double the $\mathrm{r}_{0}$ of A-1.21-237-30/2, its $\mathrm{O}_{\mathrm{Br}} \mathrm{H}$-group density is yet significantly lower. Since higher SSA of P25-B did not bring that much benefit in phenol's breakdown, its superior activity shall be the result mainly of the particle shape and not of the $\mathrm{O}_{\mathrm{Br}} \mathrm{H}$-group density alone. This means the following: the presence of $\mathrm{O}_{\mathrm{Br}} \mathrm{H}$-groups (or perhaps $\mathrm{OH}$-groups, in general) is a necessary but non-sufficient condition of achieving outstanding performance. It is likely that an extended structural order - including well-developed crystalline facets - is more essential as it restrains 
electron-hole recombination [237,238]. Its special case is when $\{001\}$ facets start to appear in the system further improving charge separation efficiency. An apparent discrepancy arises if assuming $\mathrm{O}_{\mathrm{T}} \mathrm{H}$-group density to be directly proportional to the area of these crystallographic surfaces: B-1.24710-30's $\mathrm{r}_{0}$ is the lowest out of the three B-samples, whereas it has the highest $\mathrm{O}_{\mathrm{T}} \mathrm{H}$ content. This may, however, be in accordance with the observation that an optimum value of $\{001\} /\{101\}$ ratio may exist for certain photocatalytic reactions [230].

\subsection{Hydrodynamic behavior of aqueous $\mathrm{TiO}_{2}$ suspensions}

The interaction between the dispersion medium and the polycrystalline $\mathrm{TiO}_{2}$ particulate systems can be highly influential to photocatalytic efficiency, as it was explained before in Section 2.6. Colloidal stability of aqueous titanium dioxide suspensions depends mainly on the following factors: the hydrophilicity (i.e., density of surface hydroxyl groups) of particles, their sizes, the $\mathrm{pH}$ and the ionic strength of the solution. Smaller agglomerate size provides better light absorption since larger portion of particles can be excited being directly exposed to the irradiating light [135]. Flame-made titanium dioxide samples - such as P25 - are known to be very hydrophilic. They are easily dispersed in water and form stable suspensions, in which particles do not settle even after several days. The surface charge of particles has an effect not only on the coagulation processes but influences the adsorption of different compounds as well, especially of those with ionic form. Due to electrostatic attraction or repulsion phenomena, it could be determining whether molecules can react directly on the catalyst surface or they interact with radicals released into the aqueous medium [137]. For this reason, it is worth having information on the $\mathrm{pH}$-dependence of catalyst particles' charging/coagulation behavior. By the aid of DLS, it was possible to measure zeta potential and agglomerate size, simultaneously. In Table 19, DLS results together with the most essential sample characteristics are given for all $\mathrm{TiO}_{2} \mathrm{~S}$ investigated. Here, $\mathrm{d}_{\mathrm{DLS}}$ denotes the average hydrodynamic diameter of $\mathrm{TiO}_{2}$ agglomerates while $\mathrm{pH}_{\text {IEP }}$ stands for the isoelectric point expressed in $\mathrm{pH}$ values.

\begin{tabular}{|c|c|c|c|c|c|c|}
\hline $\begin{array}{c}\text { Sample ID } \\
\text { (burner-H2: } \\
\text { vTiCl4-T } \text { TiCl4 }_{2} \text { - }\end{array}$ & $\begin{array}{l}\text { Anatase } \\
\text { content } \\
\text { (wt } \%)\end{array}$ & $\begin{array}{c}\text { Anatase } \\
\text { size / XRD } \\
(\mathbf{n m})\end{array}$ & $\begin{array}{l}\text { Activity/ro,phenol } \\
\left(\times 10^{-8} \mathbf{M s}^{-1}\right)\end{array}$ & $\begin{array}{c}\text { Frequency of } \\
\text { polyhedral } \\
\text { particles (\%) }\end{array}$ & $\begin{array}{c}\text { dpLS @ } \\
\mathrm{pH} \sim 6 \\
(\mathrm{~nm})\end{array}$ & pHIEP \\
\hline B-1.00-355-30 & 96 & 34 & $15.47 / 15.68$ & 100 & 540 & 3.5 \\
\hline B-1.24-355-30/2 & 82 & 62 & 25.70 & 96 & 560 & 2.4 \\
\hline B-1.41-355-30 & 57 & 67 & 9.77 & 90 & 820 & 2.2 \\
\hline B-1.24-710-30 & 84 & 51 & 20.39 & 97 & 610 & 3.0 \\
\hline B-1.24-355-70 & 81 & 63 & 19.35 & 97 & 620 & 4.0 \\
\hline P25-B & 89 & 27 & 15.40 & 100 & 920 & 5.8 \\
\hline
\end{tabular}

Table 19: DLS data, structural parameters and photoactivities of samples investigated. Activities indicated after a slash represent the results of degradation measurement repetitions. 
The sign of zeta potential is the same as of the excess charge of the particle that moves together with its adhered layer of counterions, and its value is proportional to the particle charge. The sign reverses at a characteristic $\mathrm{pH}$ - identified as the $\mathrm{pH}$ of isoelectric point -, where amphoteric particles do not hold net charge anymore (Figure 39).

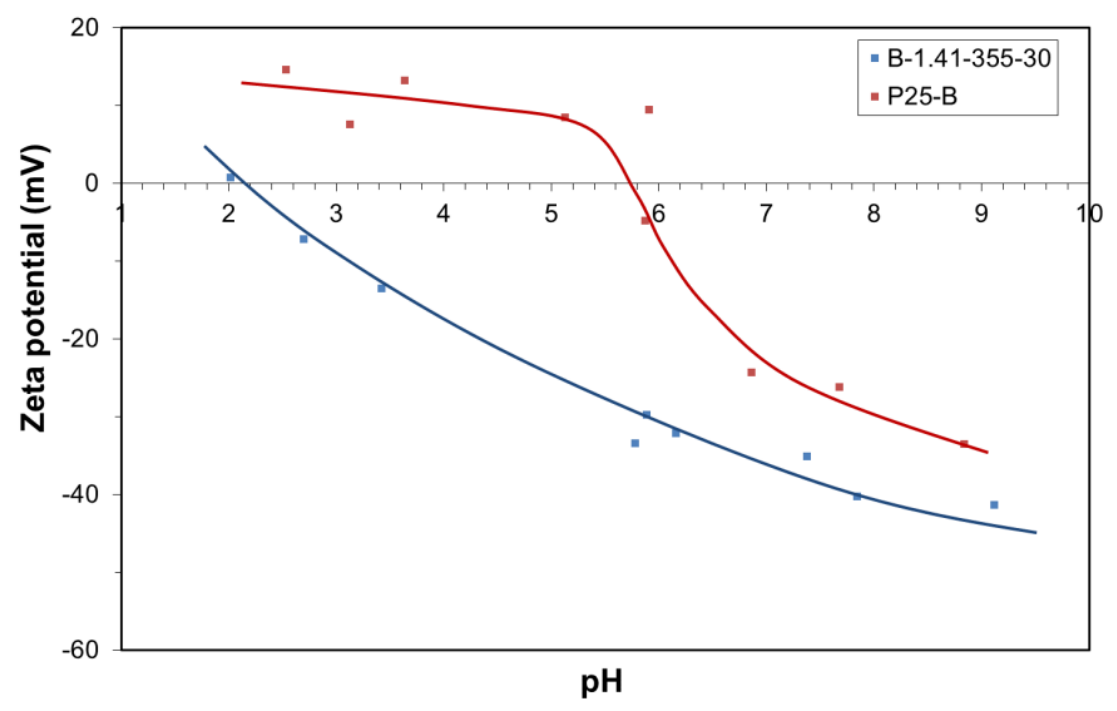

Figure 39: Zeta potential curves of sample B-1.41-355-30 and P25-B.

Based on Table 19 data, no unambiguous interdependence can be revealed between $\mathrm{pH}_{\text {IEP }}$ values and the individual structural parameters. This is somewhat anticipated because $\mathrm{pH}_{\text {IEP }}$ is collectively defined by $\mathrm{pK}_{\mathrm{a}}$-s of each hydroxyl species. Latter quantities are, in turn, dependent on multiple factors (e.g., crystalline phase, facet orientation, defect structure, etc.) potentially impacting the local electron density on hydroxyl group oxygen atoms and, therefore, the bond strength of

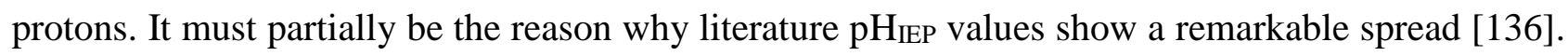
Contrary to the expectations, no correlation was found with XPS results either (previous section, Table 18). P25-B, that has exclusively the most acidic $\mathrm{O}_{\mathrm{Br}} \mathrm{H}$-groups $\left(\mathrm{pK}_{\mathrm{a}}=2.8\right)$ on its surface, possesses considerably higher $\mathrm{pH}_{\text {IEP }}$ than our two samples investigated also by DLS (B-1.24-35530/2 and B-1.24-710-30). Likewise, in the case of the latter two samples, the atomic ratio of $\mathrm{O}_{\mathrm{T}} \mathrm{H}-$ groups $\left(\mathrm{pK}_{\mathrm{a} 1}=4.5 ; \mathrm{pK}_{\mathrm{a} 2}=8.0\right)$ reaches more than half of the bridging ones while their $\mathrm{pH}$ IEP-s equal or stay below 3. As described above, acid strength of $\mathrm{OH}$-groups might be affected by their electronic environment to some extent, but to our knowledge, no research work has been published so far that is targeted on quantifying such phenomenon. Whatever the root cause is, significantly smaller $\mathrm{pH}_{\text {IEP }}$ values (2.2-4.0) were measured for our $\mathrm{TiO}_{2} \mathrm{~S}$ than for $\mathrm{P} 25-\mathrm{B}$, which falls around 5.8, being close to the average value of titanium dioxides ( 5.6) [136]. Low isoelectric points may predict a good catalytic performance. For instance, Gumy et al. [137] found that photocatalysts with $\mathrm{pH}_{\mathrm{IEP}-\mathrm{S}}<3$ were the most active ones at phenol decomposition. This observation is consistent with the 
expectations assuming a situation, when the aggregation level of $\mathrm{TiO}_{2}$ dispersions was the only parameter determining the photocatalytic efficiency of different catalyst systems - i.e., all their characteristics were identical except $\mathrm{pH}$ IEP-s. Before adding phenol to the suspensions $\left(\mathrm{c}_{\mathrm{TiO} 2}=1 \mathrm{~g} / \mathrm{L}\right)$, we occasionally measured their initial $\mathrm{pH}$-s and obtained values between 4.8 and 6.6 for our samples while we got 5.1 for P25-B. It can be calculated that, under such circumstances, the dissociation of phenol $\left(\mathrm{K}_{\mathrm{a} \text {,phenol }}=1.6 \times 10^{-10}\right)$ has negligible contribution. By contrast, as the photocatalytic reaction progresses, more and more acidic compounds start to appear lowering the $\mathrm{pH}$ of the dispersion. Although $\mathrm{pH}$ was not monitored during and after phenol degradation experiments, it could unlikely

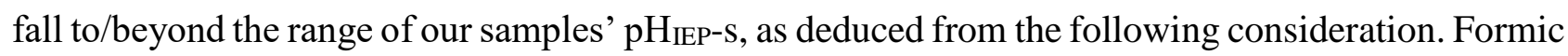
acid - being the strongest of all potential carboxylic intermediates - could lower the pH only to $~ 3.5$ 3.6, if it had 6-times higher concentration than phenol's initial concentration $\left(5 \times 10^{-4} \mathrm{M}\right)$. Since formic acid represents only one stage of the entire reaction chain, whose components are continuously transforming into one another in certain sequences, it will never be present in such high quantity. Accordingly, most of our samples characterized by DLS (apart maybe from B-1.24-355-70) shall preserve satisfactory suspension stability throughout the whole degradation. This might not be true for catalysts having $\mathrm{pH}_{\mathrm{IEP}-\mathrm{S}}$ around 5-6 (like P25-B), because in our model reaction system, they can get close to the aggregated state right at the beginning.

As seen in Table 19, $\mathrm{pH}_{\mathrm{IEP}}$ results do not fully correlate with the average hydrodynamic diameters $\left(\mathrm{d}_{\mathrm{DLS}}\right)$ that were recorded at $\mathrm{pH} \sim 6$. This originates from the different particle size distribution and chemical nature of the individual polycrystalline systems, eventually governing the $\mathrm{pH}$-dependent surface charge formation. B-sample agglomerates were determined to be within 540 and $620 \mathrm{~nm}$, which sizes are considerably smaller than that of P25-B (900 nm). The only exception is B-1.41-355-30. Even though it has the lowest $\mathrm{pH}_{\text {IEP }}$, a $\mathrm{d}_{\mathrm{DLS}}$ of $820 \mathrm{~nm}$ was measured. This relatively high value might be related to its unique chemical quality: it was synthesized under the most reductive conditions (with a $\mathrm{H}_{2}-\mathrm{O}_{2}$ molar ratio of 1.41 ), as indicated by its blueish color resulting from the presence of surficial and subsurface oxygen vacancies. For sake of curiosity, test tube sedimentation experiments were also conducted by suspending catalysts used at DLS (+ sample B-1.24-178-30) in pure MilliQ-water. $\mathrm{TiO}_{2}$ concentrations of these dispersions were set to $1 \mathrm{~g} / \mathrm{L}$, therefore their $\mathrm{pH}$-s were estimated to be in the same range as determined before photocatalytic runs. Observations taken here are in good agreement with DLS data. In general, almost all of our samples have shown remarkable suspension stability, even after 72 hours of storage (Figure 40). B-1.24-355-30/2, our best photocatalyst, displayed the highest stability as having the second smallest $\mathrm{d}_{\mathrm{DLS}}$ value at around $\mathrm{pH}$ 6. On the other hand, sample B-1.41-355-30 (third tube from the left) has discernibly started coagulating already after 3 hours. Interestingly, despite its highest $\mathrm{d}_{\mathrm{DLS}}$ and $\mathrm{pH}_{\mathrm{IEP}}$, the turbidity of P25-B's suspension has not dropped so much behind the average. 


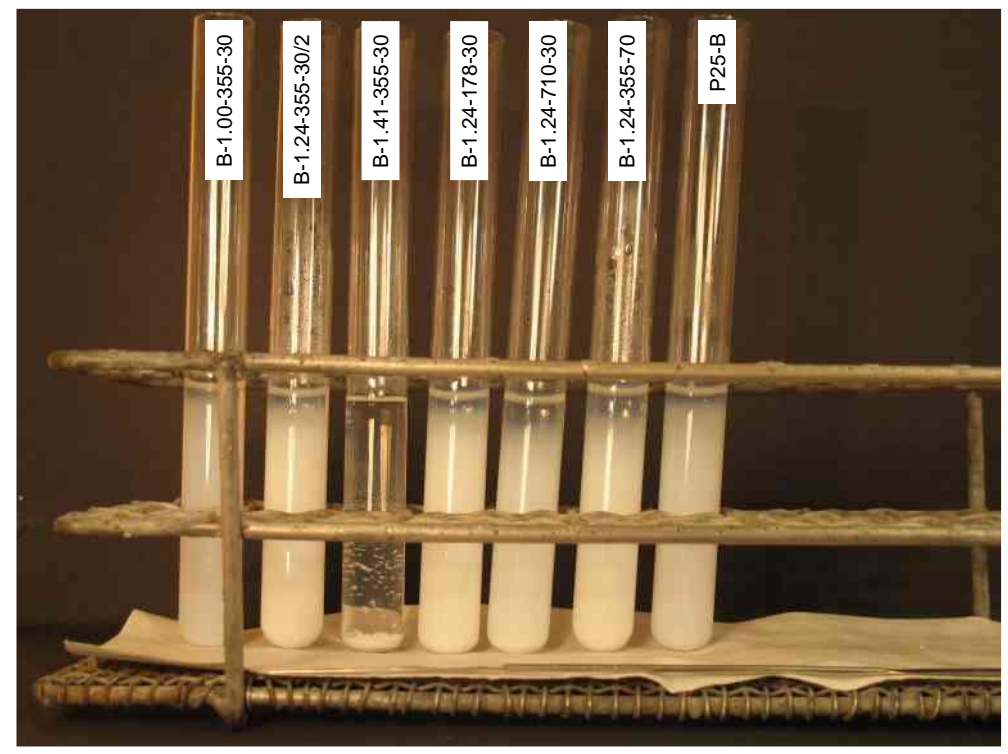

Figure 40: Image on several $\mathrm{TiO}_{2}$-MilliQ-water colloid systems $\left(\mathrm{c}_{\mathrm{TiO} 2}=1 \mathrm{~g} / \mathrm{L}\right)$ after 72 hours of undisturbed sedimentation. Sample IDs are indicated on the upper part of the tubes.

It was pointed out in this chapter that the actual condition/stability of the titanium dioxide aqueous dispersions can play an essential role in heterogeneous photocatalytic processes. Most importantly, if $\mathrm{pH}_{\mathrm{IEP}}$ of a $\mathrm{TiO}_{2}$ sample is located in an advantageous range of the $\mathrm{pH}$ scale, a desired performance may be sustained all the way up to the complete mineralization without using any $\mathrm{pH}$ active additive.

\subsection{Oxygen consumption results}

Despite oxygen consumption data could greatly contribute to the characterization of catalytic performances, there are not so many articles in the literature [239] that investigate $\mathrm{TiO}_{2}$-mediated photocatalytic decomposition reactions in terms of this quantity. In such model reaction systems like ours, oxygen acts as the primary electron acceptor, therefore, the rate of its adsorption and the efficiency of electron scavenging (i.e., interfacial charge transfer between $\mathrm{O}_{2}$ molecules and $\mathrm{TiO}_{2}$ particles) undoubtedly determine photocatalytic efficiency. Although we were not able to directly characterize these two processes, the measured oxygen depletion rate must incorporate their impacts as well.

It seemed reasonable to use the same slurry photoreactor design at these measurements like what we applied so far. For this purpose, only a slight modification was necessary to be done on it to ensure its gastight sealing and to have an oxygen sensor receptacle integrated (Figure 41). In this 
series of experiments, the most representative B-samples and the two different P25 catalysts were tested. All their relevant data are collected in Table 20.
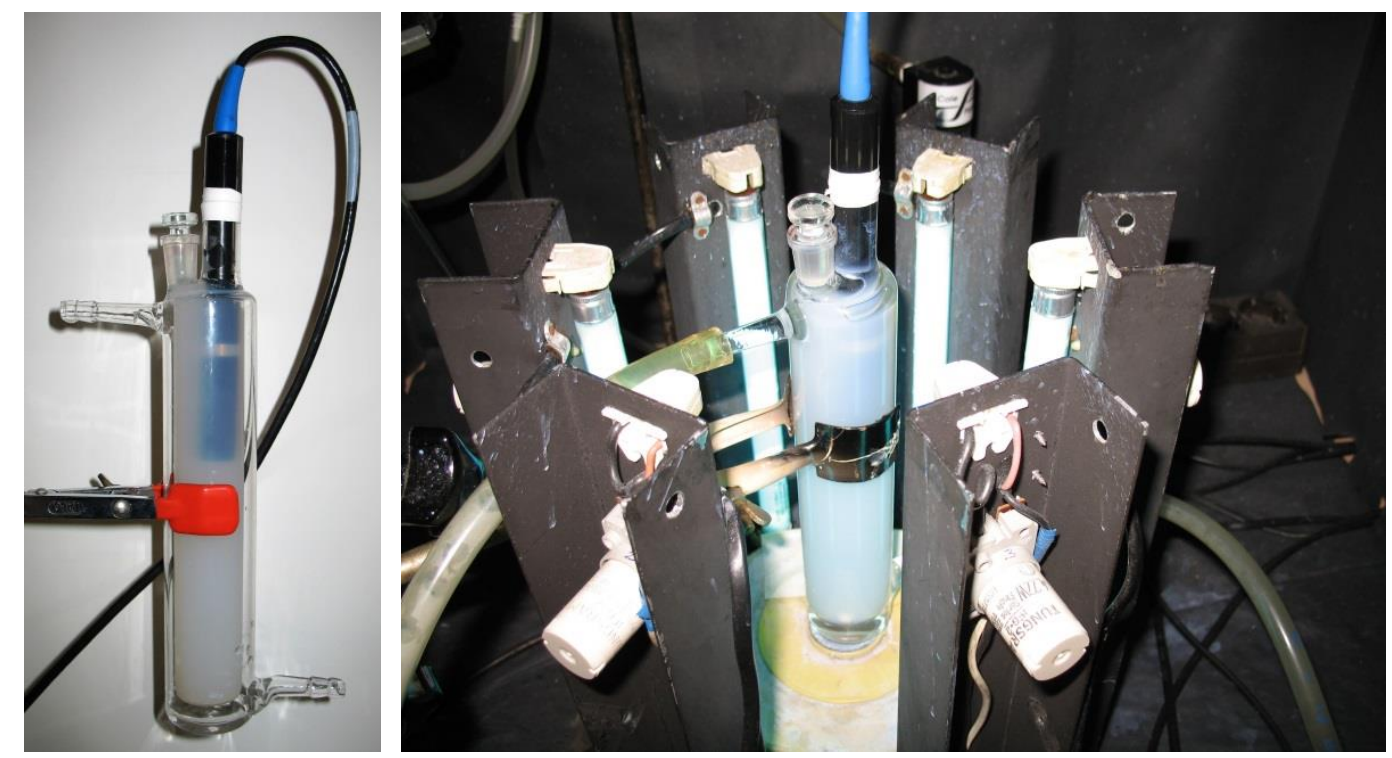

Figure 41: Experimental setup of oxygen consumption measurements. Left image: photoreactor sealed with a gastight plug and the oxygen-sensitive biamperometric cell (black plastic tube) is immersed in the $\mathrm{TiO}_{2}$ suspension. Right image: the reactor is placed between six operating UVlamps.

In Figure 42, oxygen consumption curves of the photocatalytically most (B-1.24-355-30/2) and least (B-1.41-355-30) efficient samples are displayed along with the data of P25-A and B. Based on measurement repetition results of the latter two catalysts, good reproducibility of the experiments becomes evident.

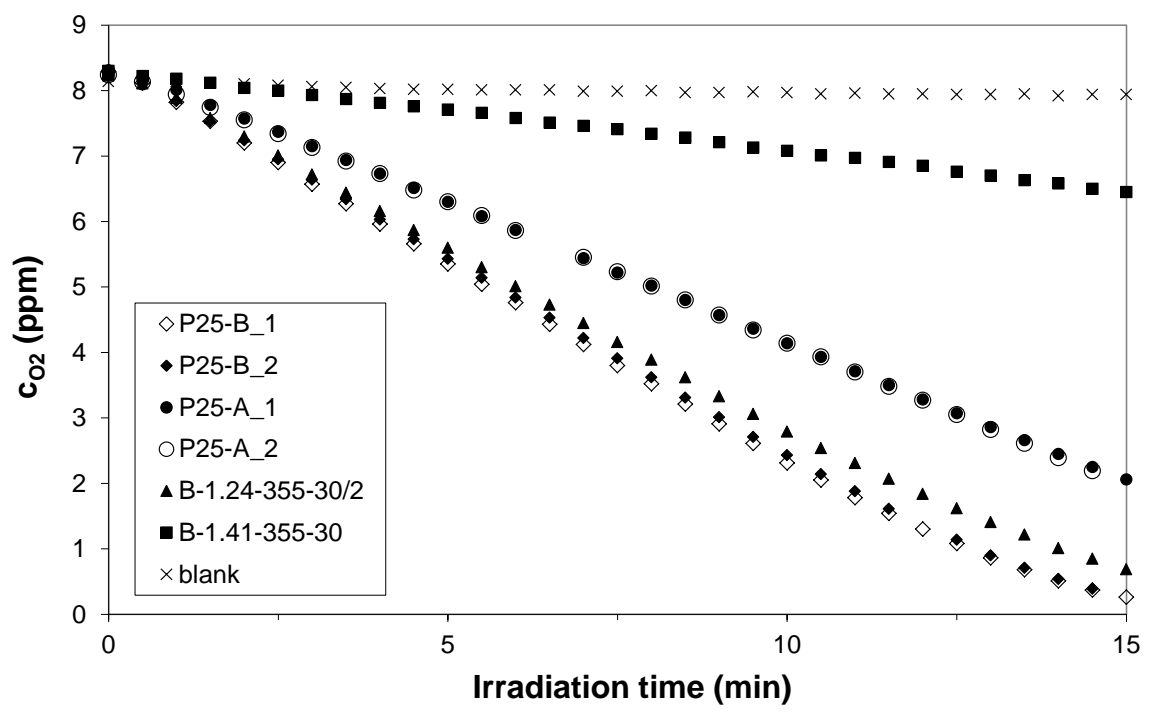

Figure 42: $\mathrm{O}_{2}$-consumption curves of the most representative samples investigated. 
Since there is no oxygen supplement in the system, its concentration rapidly approaches zero, especially at samples with the highest photocatalytic activity. Like it was stated before, the $\mathrm{TiO}_{2}$ content had to be reduced to the one fifth $(0.2 \mathrm{~g} / \mathrm{L})$ of the one used at the conventional phenol decomposition reactions in order to slow down the rate of oxygen consumption. As a short plateau was present at the beginning of these curves (most likely due to the initial warm-up stage of the light sources while they are providing lower photon flux), an empirical function has been fitted on the data points between 2 and 15 minutes. The slope of this function at $\mathrm{t}=2 \mathrm{~min}$ was calculated and considered to be the measure of the initial oxygen consumption rate $\left(\mathrm{r}_{0, \mathrm{O} 2}\right)$.

\begin{tabular}{|c|c|c|c|c|c|c|}
\hline $\begin{array}{c}\text { Sample ID } \\
\text { (burner-H2:O2- } \\
\text { VTiCl4- } \mathrm{T}_{\mathrm{TiCl} 4} \text { ) }\end{array}$ & $\begin{array}{c}\text { Anatase } \\
\text { content } \\
(w t \%)\end{array}$ & $\begin{array}{c}\text { Anatase } \\
\text { size / XRD } \\
(\mathbf{n m})\end{array}$ & $\begin{array}{l}\text { SSA / } \\
\text { BET } \\
\left(\mathrm{m}^{2} / \mathrm{g}\right)\end{array}$ & $\begin{array}{c}\text { Frequency of } \\
\text { polyhedral } \\
\text { particles }(\%)\end{array}$ & $\begin{array}{c}\text { Activity } \\
\text { /ro,phenol } \\
\left(\times 10^{-8} \mathrm{Ms}^{-1}\right)\end{array}$ & $\begin{array}{l}\mathrm{O}_{2} \text {-consumption/ } \\
\mathrm{r} 0,02\left(\times 10^{-8} \mathrm{Ms}^{-1}\right)\end{array}$ \\
\hline B-1.00-355-30 & 96 & 34 & 45 & 100 & $15.47 / 15.68$ & 29.4 \\
\hline B-1.24-355-30/2 & 82 & 62 & 21 & 96 & 25.70 & 28.9 \\
\hline B-1.41-355-30 & 57 & 67 & 19 & 90 & 9.77 & 5.9 \\
\hline B-1.24-178-30 & 80 & 59 & 24 & 95 & 21.42 & 28.2 \\
\hline B-1.24-710-30 & 84 & 51 & 27 & 97 & 20.39 & 24.9 \\
\hline B-1.24-355-70 & 81 & 63 & 22 & 97 & 19.35 & 22.3 \\
\hline P25-A & 79 & 27 & 50 & 85 & 11.80 & 22.1 \\
\hline P25-B & 89 & 27 & 50 & 100 & 15.40 & 31.6 \\
\hline
\end{tabular}

Table 20: Structural parameters, photoactivities and $\mathrm{O}_{2}$-consumption rates of samples investigated.

When plotting $\mathrm{r}_{0, \text { phenol }}$ against $\mathrm{r}_{0, \mathrm{O} 2}$ values for each sample (Figure 43 ), no clear correlation between these two data sets is seen. This fact is, however, not that surprising since oxygen is consumed for the transformation of all oxidizable species getting close enough to (or being adsorbed on) $\mathrm{TiO}_{2}$ particles. Phenol is only one amongst these many substances. Moreover, it must compete for unreacted dissolved radicals while most of its derivatives can easily be adsorbed on titania surfaces and thus, do not have such oxidation rate limiting factors like radical diffusion.

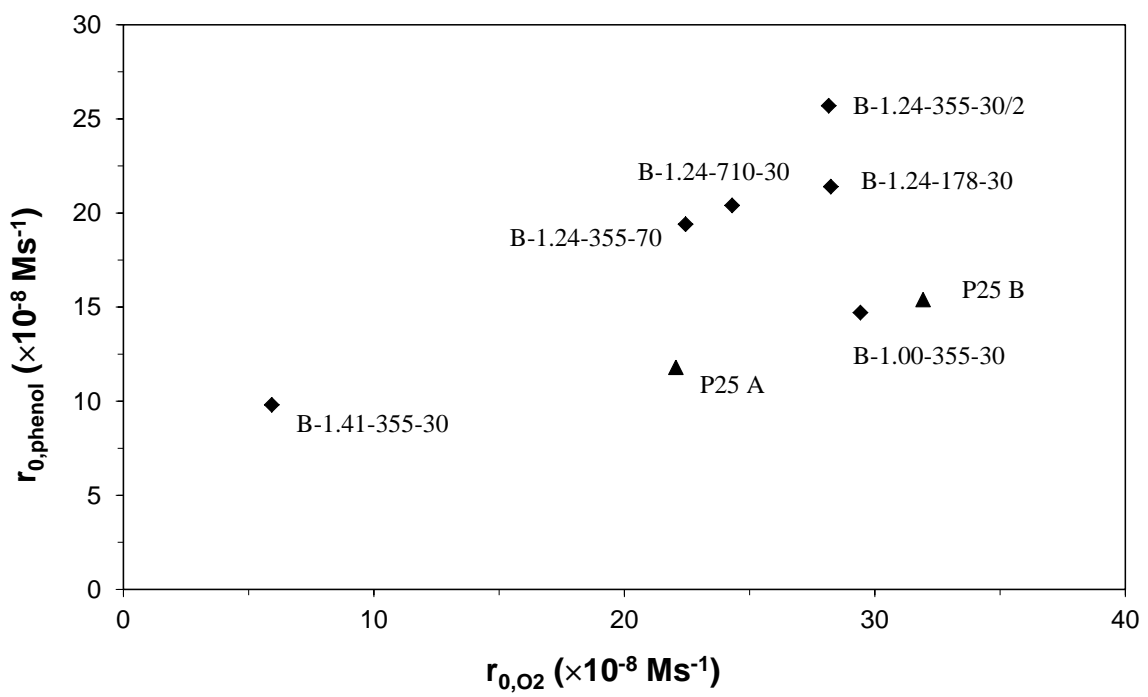

Figure 43: $r_{0, \text { phenol }}$ data are plotted against the corresponding $r_{0,02}$ values. 
A significantly different situation is observed if each $\mathrm{r}_{0,02}$ is normalized by the respective specific surface area and catalyst loading (i.e., $\mathrm{r}_{0,02} /\left(\mathrm{SSA} \times \mathrm{c}_{\mathrm{TiO}}\right)$ ). The resulting quantity practically describes the general photocatalytic quality of $\mathrm{TiO}_{2} \mathrm{~S}$ as it gives the net molar amount of molecular oxygen converted over unit surface per unit time $\left(\mathrm{mol} \mathrm{s}^{-1} \mathrm{~m}^{-2}\right)$. If the previous graph is reproduced by using the normalized $\mathrm{r}_{0, \mathrm{O} 2}$, a quasi-linear behavior is obtained (Figure 44) which justifies the validity of the following statement: initial phenol (and perhaps other non-adsorbing compounds') decomposition rate may be an adequate measure for the comparison of aqueous phase photocatalytic performances of different $\mathrm{TiO}_{2} \mathrm{~s}$. As long as the amount of well-adsorbing intermediates is negligible, phenol degradation rate is defined mostly by the radical production efficiency of catalysts. This feature is, of course, still affected by the SSA to some extent, since more substance (e.g., oxygen) can be bound and more photons can be absorbed by a larger surface. Accordingly, the above normalization yields a surface area-independent photocatalytic efficiency that obviously shows correlation with phenol degradation rate in such an early phase of the reaction, when the concentration of intermediates has just started to grow. It is important to note again, that the majority of our samples investigated here are situated far above P25-B on this scale, which catalyst is still known as one of the most efficient ones that can be purchased in the market.

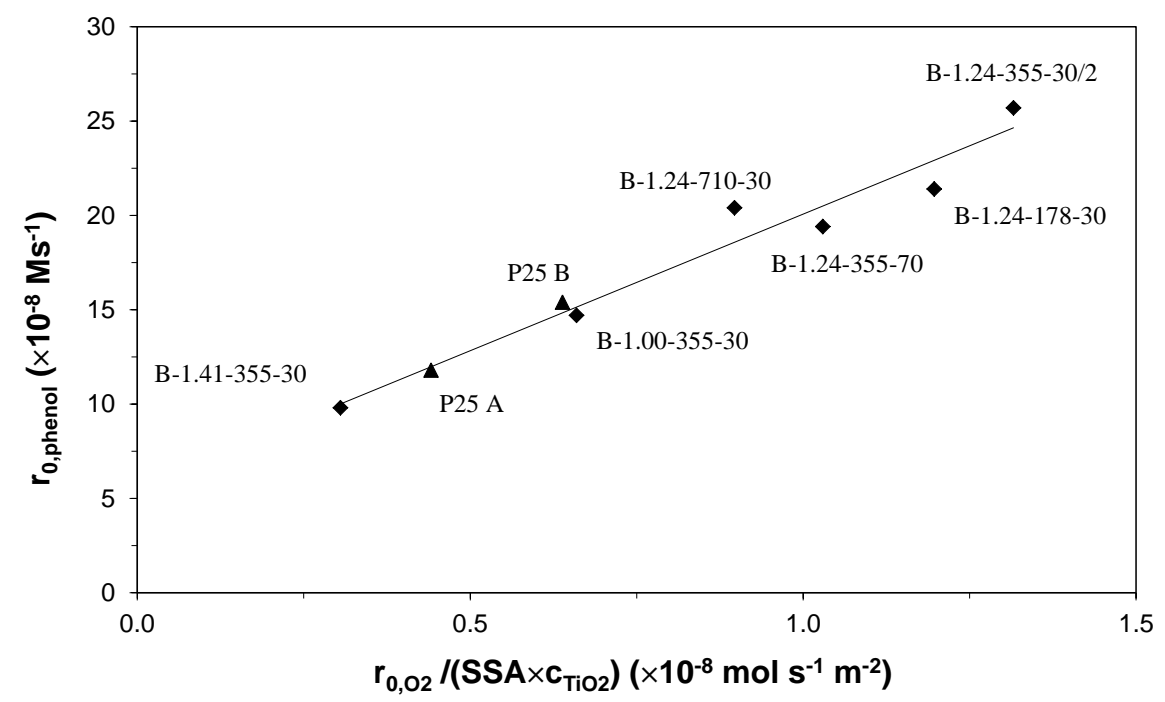

Figure 44: $\mathrm{r}_{0, \text { phenol }}$ data are plotted against the corresponding normalized $\mathrm{r}_{0, \mathrm{O} 2}$ values.

Dissolved oxygen monitoring can be a unique tool for routine comparison of aqueous phase photocatalytic activities. It is very simple, fast and cheap. Therefore, if mechanisms of a model reaction are known and there is no need of tracking intermediate concentrations, it may supersede such labor-intensive and expensive techniques, like TOC and chromatography. 


\subsection{Mineralization efficiencies and intermediate studies}

As the first step of phenol's photocatalytic breakdown, it is converted into dihydroxy intermediates, PC and HQ. When these compounds are further oxidized, different aliphatic oxygenated substances and finally carbon dioxide evolve. Like it was stated before, phenol is transformed into PC and HQ almost exclusively by OH-radical insertion. From the point when intermediates appear in the suspension, their adsorption and subsequent decomposition suppress phenol degradation since less and less radicals reach the parent molecules staying farther away from catalyst particles.

Regarding the complete removal of toxic organics from polluted water, it might be necessary to know the quality and the concentration of intermediates formed from the primary substrate. This provides deeper understanding on reaction mechanisms and could give a better chance to select the optimal photocatalyst for a given target organic compound. Besides HPLC-based concentration profiles of phenol, PC and HQ, total organic carbon (TOC) decay curves are also determined for three $\mathrm{TiO}_{2} \mathrm{~s}$, including B-1.00-355-30, B-1.24-355-30/2 and P25-B (Figure 45). TOC analysis has proven to be a valuable complementary technique here as it reveals the global rate of phenol mineralization in the same reaction systems as used at the original decomposition experiments. It was hence possible to transfer the information content of the left graphs to TOC plots in Figure 45, simply by expressing the actual molar concentrations in ppm values. "TOC aromatics" in the caption denotes the total quantity of all major aromatic substances (phenol, HQ, PC) measured by HPLC, therefore its subtraction from "TOC measured" yields the amount of undetected, mostly aliphatic intermediates. 

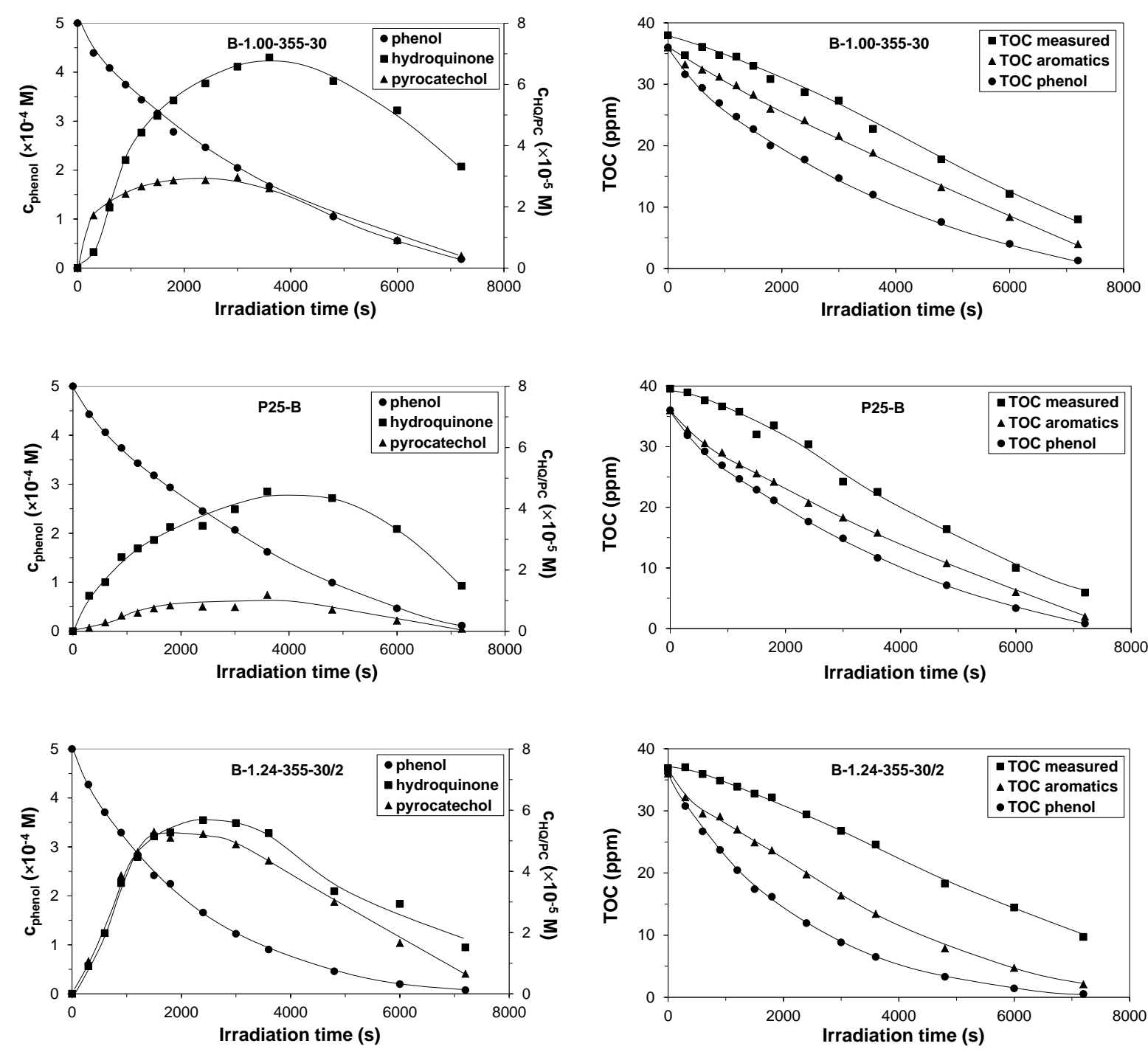

Figure 45: Left column: concentration profiles of phenol, hydroquinone (HQ) and pyrocatechol (PC) during the photocatalytic decomposition of phenol using sample B-1.00-355-30, B-1.24-355-30/2 and P25-B.

Right column: variation of the measured and calculated (for phenol, HQ and PC) total organic carbon (TOC) contents at the above reactions.

It shall be noted that, though, this collective TOC representation of all measured quantities is quite expressive, the transformation of aromatic intermediates' aqueous phase concentrations to TOC values introduces a certain extent of error into the calculation. Namely, it does not take into account the adsorbed amounts of HQ and PC. Therefore, TOC data of HQ, PC and of the aliphatic compounds are somewhat uncertain while "TOC phenol" (being a non-adsorbing material) and "TOC measured" are still considered correct. Nevertheless, it will not lead to any misinterpretation when comparing P25-B with B-1.00-355-30, as their SSAs and phenol degradation rates are almost identical, while tendencies observed at sample B-1.24-355-30/2 can clearly be traced back to the significant differences of just the above properties.

According to the data included in Figure 45 and in Table 21, P25-B and B-1.00-355-30 display very similar catalytic performances. The latter was consciously selected into the group of the most 
representative flame-made samples on which a more extended investigation has been carried out, since it has almost the same material properties than P25-B (Table 21). Their polyhedral particle contents, particle sizes and SSAs are nearly identical, only their phase compositions differ slightly. Based on this observation, our hope was exactly to find any similarity in photocatalytic behaviors. Measurement results have shown indeed, that both data pairs of phenol decomposition and of oxygen consumption rates fall close to one another. Unsurprisingly, such a resemblance is found between the overall TOC decay characteristics, however, concentration profiles of the two types of intermediates (aromatic and aliphatic) look a bit differently. It turns out from both the degradation and the TOC curves that HQ and PC concentrations are smaller for P25-B at most sampling points. Nevertheless, this small difference continuously vanishes by the end of the reaction thus indicating roughly identical overall mineralization efficiencies for both $\mathrm{TiO}_{2} \mathrm{~s}$. Our best photocatalyst (classified by its initial phenol decomposition rate), B-1.24-355-30/2, displays a rather different nature of operation than the previously treated two samples. The most conspicuous example is the constantly larger amount of aromatic and aliphatic substances. Although the former kinds of materials eventually reach similar concentrations like it was detected for the other two catalysts, the curve "TOC measured" runs the highest of all throughout the experiment. Interestingly, PC's liquid phase concentration stays closer to that of HQ (with less preferred adsorption). This may be interpreted in the following way: B-1.24355-30/2 has ca. 1.65-times higher initial phenol degradation rate which results in a much faster intermediate production, meaning aromatic as well as aliphatic ones. Consequently, as B-1.24-355$30 / 2$ has less than half the SSA of the other two samples, saturation of available adsorption sites starts much earlier, enabling the otherwise well-adsorbing PC to be preceded by carboxylic acids that are even more capable of binding to $\mathrm{TiO}_{2}$. On the other hand, despite the high surface coverage, radical production efficiency of B-1.24-355-30/2 is preserved longer which makes this catalyst superior to others in decomposing phenol. Regardless of its limiting low specific surface area, B-1.24-355-30/2 could yet acceptably compete with other samples concerning total mineralization efficiency, as proven by TOC measurements.

\begin{tabular}{|c|c|c|c|}
\hline $\begin{array}{c}\text { Sample ID } \\
\text { (burner-H2:O2- } \\
\text { VTiCl4- }_{\text {TiCl4) }}\end{array}$ & $\begin{array}{l}\text { Activity/ro,phenol } \\
\left(\times 10^{-8} \mathrm{Ms}^{-1}\right)\end{array}$ & $\begin{array}{l}\mathrm{O}_{2} \text {-consumption/ } \\
\mathrm{r}_{0,02}\left(\times 10^{-8} \mathrm{Ms}^{-1}\right)\end{array}$ & $\begin{array}{l}\text { "TOC measured" } \\
\text { @ } 7200 \mathrm{~s}(\mathrm{ppm})\end{array}$ \\
\hline B-1.00-355-30 & $15.47 / 15.68$ & 29.4 & 8.0 \\
\hline B-1.24-355-30/2 & 25.70 & 28.9 & 11.4 \\
\hline B-1.41-355-30 & 9.77 & 5.9 & $\mathrm{n} / \mathrm{a}$ \\
\hline B-1.24-178-30 & 21.42 & 28.2 & $\mathrm{n} / \mathrm{a}$ \\
\hline B-1.24-710-30 & 20.39 & 24.9 & $\mathrm{n} / \mathrm{a}$ \\
\hline B-1.24-355-70 & 19.35 & 22.3 & $\mathrm{n} / \mathrm{a}$ \\
\hline P25-A & 11.80 & 22.1 & $\mathrm{n} / \mathrm{a}$ \\
\hline P25-B & 15.40 & 31.6 & 5.9 \\
\hline
\end{tabular}

Table 21: Photocatalytic activities, $\mathrm{O}_{2}$-consumption rates and aggregate TOC values measured at the end of photocatalytic runs (where applicable). 
Based on the above elucidation, one may have the choice to select a certain quality of $\mathrm{TiO}_{2}$ according to a given application-specific requirement: sometimes the most important aspect might be either to remove the most toxic compound (e.g., phenol) as fast as possible (specimen B-1.24-355$30 / 2$ is chosen), or to provide the highest rate for the total mineralization of organics present in contaminated water (P25-B is selected). 


\section{Summary}

We have successfully developed two $\mathrm{H}_{2}$-air-fed diffusion flame reactors (Burner-A and -B) and all peripheral elements of the complete experimental setup (quartz chimney, $\mathrm{TiO}_{2}$-collector, precursor vial) that were collectively demonstrated to be suitable for flame-assisted synthesis of nanoparticulate $\mathrm{TiO}_{2}$ catalysts. This synthesis setup ensured stable and well-controllable operating conditions which eventually granted the desired level of reproducibility with respect to material characteristics and to photocatalytic activities of the products.

After recognizing the technical limitations of Burner-A, its construction has gone through some improvements leading to the design of Burner-B. The opportunity of having these somewhat different reactors in hand provided the chance to compare the tendencies of catalysts' primary structural parameter changes as the function of each operational parameter set. This comparison significantly contributed to our understanding of those elementary rules of particle dynamics that finally determine the material properties of $\mathrm{TiO}_{2}$ samples. Concerning this particular topic, our observations - typically showing good agreement with literature deductions - are listed in the following points, classified by the type of the burner at which they were taken.

Burner- $A / B$ : It has indirectly been verified by experimental data that rutile clusters do not exist at the very beginning of particle formation but they evolve from anatase clusters. This statement is proven by the fact that rutile particle sizes are always bigger than those of anatase belonging to the same sample. For a special exception, see the next point.

Burner-B: It was pointed out that setting up higher and higher $\mathrm{H}_{2}-\mathrm{O}_{2}$ molar ratios - hence higher flame temperatures - will yield gradually bigger particle sizes, higher degrees of dispersity and increasing rutile contents. Only two samples, located at the uppermost segment of the scale, exhibited downward trend in rutile particle sizes. This phenomenon is attributed to the emerging $\mathrm{O}_{2}$-lean environment that creates oxygen vacancies in the $\mathrm{TiO}_{2}$ crystal lattice. These defects are known to facilitate the anatase $\rightarrow$ rutile phase transformation and consequently, rutile particles start to evolve from smaller and smaller clusters than under less reductive circumstances.

Burner-B: At the initial stages of precursor feed rate increase, anatase particle sizes get bigger and anatase content does not start growing, in harmony with Burner-A findings. This behavior can be explained by a relatively constant gas phase precursor concentration that is preserved up to a certain rate. Within this lower range, in addition, flame temperature is gradually increasing as setting up higher values, because the number of reacting $\mathrm{TiCl}_{4}$ molecules per unit time and per unit volume is growing. Passing beyond that feed rate threshold - which could not fully be reached at Burner-A -, the precursor-air mixture gets leaner and sample property changes start to obey the initial expectations. Namely, particle sizes shrink and the anatase content increases since the decreasing in- 
flame particle residence time becomes the dominating factor. This effect is further enhanced by a certain extent of flame temperature drop, arising partially from precursor dilution and from the intensified heat dissipation driven by the increasing volumetric flow.

Burner-A: Mutual perturbing actions of the individual gas streams $\left(\mathrm{H}_{2}\right.$, air and precursor intake $)$ were observed through distorting the results of sample characteristics that could theoretically be anticipated for an "ideal" experimental system (where all variables are completely independent from one another within their entire functional range). This influence has shown large dependency on several factors, like burner geometry, absolute values of the individual flow rates and their ratios.

Burner-A: Under certain conditions, the chemical transformation of $\mathrm{TiCl}_{4}$ liberates such an extent of thermal energy that is comparable to the enthalpy content of the flame. It may, thus, considerably influence the spatial temperature distribution of the reaction zone. Accordingly, controllability of the synthesis procedure can highly be improved by choosing an appropriate precursor gas phase concentration range.

Burner-B: An attempt has been made to study the standalone impact of increasing the gas phase concentration of the precursor while setting all other synthesis parameters (i.e., gas flow rates) to values at which our best catalyst (B-1.24-355-30/2) was produced. This was achieved simply by warming the $\mathrm{TiCl}_{4}$-container up (in two runs, first to $50^{\circ} \mathrm{C}$ and then to $70^{\circ} \mathrm{C}$ ) so that precursor evaporation intensified. Such an action is basically supposed to increase the rate of molecular/intercluster collisions and the flame temperature itself. Interestingly, though the resulting variation of each primary material property (particle size, phase composition) stayed within the margins of measurement error, photocatalytic activity displayed a $c a$. $25 \%$ drop between sample B1.24-355-30/2 and B-1.24-355-70. In order to reveal the reason behind this phenomenon, further investigations on material/surface characteristics have later been conducted.

Burner-B: As the last one of all available synthesis parameter change options, effects of the overall gas flow rate modification have also been examined. For this purpose, two individual syntheses have been performed, where the hydrogen and the air flow rates (except the one for precursor feed) were simultaneously altered to obtain a $20 \%$ higher and then a $20 \%$ lower overall flow rate relative to that of the most efficient catalyst (B-1.24-355-30/2). Owing to this adjustment, the $\mathrm{H}_{2}-\mathrm{O}_{2}$ molar ratio and the precursor mass flow were unchanged. Furthermore, our calculations showed that no significant influence was exerted on the flow characteristics of the reaction zone either. Under these circumstances, only the flame temperature is supposed to be varied as smaller or bigger amount of fuel is combusted in a unit time. Sample analysis revealed that by increasing the overall flow rate, rutile content and rutile particle size gets bigger indeed, however, anatase grain size does not show a similar tendency along the series. Neither does photocatalytic activity, which decreased by an approximately $19 \%$ in both directions of adjustment. 
Besides the development the flame synthesis method, an important goal of the present work was also to understand which material characteristics play the most decisive role in defining photocatalytic efficiencies of $\mathrm{TiO}_{2}$ samples. This knowledge could namely offer the opportunity for deliberate engineering of photocatalysts with the highest possible activities, if the entire synthesis parameters-sample properties interrelation system is suitably characterized. Systematic variation of Burner-A synthesis parameters (that is, the $\mathrm{H}_{2}-\mathrm{O}_{2}$ molar ratio and the precursor feed rate) provided two sets of catalysts whose activities have been then correlated with their basic material properties. Based on the data we concluded, that neither particle size nor phase composition alone can be accounted for such a big activity difference that was measured between samples of both experimental series. The most conspicuous examples are represented by specimens A-1.21-237-30 and A-0.91237-30. Even though they practically have the same anatase content and only a $5 \mathrm{~nm}$ difference in their average anatase diameter, photocatalytic activity of A-0.91-237-30 is 2.4-times higher than that of A-1.21-237-30. Similar extents of differences were also found at those Burner-A catalysts, which were manufactured by precursor feed rate adjustment. Our later TEM investigations finally provided the most feasible explanation on these observations. It turned out that specimens having more polyhedral grains usually display better performance compared to samples that consist rather of spherical particles. The phenomenon of particle shape-dependent activity is well-known in the field of heterogeneous catalysis and it can be attributed to the different surface area ratios of specific crystalline facets.

A key question might be that which element of the synthesis parameters and how it controls grain morphology. A clear answer can be given after analyzing the two primary sets of B-sample data: the ones belonging to the precursor feed rate and to the $\mathrm{H}_{2}-\mathrm{O}_{2}$ molar ratio adjustment. It appears that the formation of polyhedral particles favors lower flame temperatures and shorter reaction zone residence times. These tendencies are not observable at Burner-A series, partially due to an inadequate experimental design $\left(\mathrm{H}_{2}-\mathrm{O}_{2}\right.$ molar ratio regulation) and to the unforeseen variation of precursor's gas phase concentration within the actual operational flow rate range of its carrier branch.

At an early stage of our studies, five A-samples (produced by precursor feed rate adjustment) and P25-B were subjected to gas phase photocatalytic decomposition experiments to get a better insight into the working mechanisms of our catalysts and to understand the origin of their diverse catalytic efficiencies. For this purpose, breakdown characteristics of two gaseous substrates methanol and acetaldehyde - have been investigated. Despite the fundamentally different physicochemical circumstances existing in aqueous and in gas phase, methanol degradation rates of the individual samples follow nearly the same order as obtained for phenol. It equally means that photocatalytic activities are bigger for samples containing more polyhedral particles, while at acetaldehyde decomposition, a vague but opposite trend appears. It is reasonable to suppose that 
above similarities stem from a somewhat comparable degradation mechanism of these two model substrates. Phenol is known as a poorly adsorbing compound due to its rather hydrophobic nature, so its decomposition is governed mostly by free radicals that successfully reach phenol molecules without reacting with other species on the way. Although methanol originally tends to anchor to $\mathrm{TiO}_{2}$ surfaces in gas phase, the excessive amount of water in the system and the gradual appearance of its well-adsorbing derivatives fully hindered its adsorption all along the reaction. Therefore, methanol molecules - just like at phenol's case - were essentially degraded by radicals. These interrelations led us to postulate that polyhedral particles have an appreciably higher efficiency in producing free radicals than spherical ones. By contrast, hole-assisted mechanism is supposed to have a more significant contribution in the degradation of acetaldehyde, where only a lesser correlation was found with polyhedral particle content. Instead, based on the evolution of rate curves, bigger SSA appeared to be advantageous in preserving higher activity.

Although photocatalytic performances of Burner-A samples have shown certain correlation with particle morphology, none of B-samples' structural properties alone (including shape, phase composition and size) displayed a clear relationship with activities. Namely, these characteristics collectively determine photocatalytic efficiencies, but with varying extent of contribution for different cases. Moreover, their impacts often manifest themselves indirectly, through such factors like chemical quality of catalyst surfaces. To capture the differences of particles' chemical nature, a few A- and B-samples plus P25-B were subjected to X-ray photoelectron spectroscopy (XPS) measurements. Ti $2 \mathrm{p}$ and $\mathrm{O}$ 1s peaks were quantitatively characterized for each specimen to enable comparison of $\mathrm{Ti}^{4+} / \mathrm{Ti}^{3+}$ and of hydroxyl/oxide (or lattice) type oxygen surface atomic ratios, respectively. It was concluded from the results that $\mathrm{Ti}^{3+}$-related surface states are not present in any of the samples. Additionally, apart from $\mathrm{OH}$-group peaks, two further $\mathrm{O}$ 1s contributions were identified on the low binding energy side of the lattice oxygen signal. The origin of these components could not be ascertained from the literature, therefore they were not involved in any assessment. While the aggregate atomic ratio of the two types of $\mathrm{OH}$-groups (terminal and bridging) did not correlate to any $\mathrm{TiO}_{2}$ property, the terminal ones $\left(\mathrm{O}_{\mathrm{T}} \mathrm{H}\right)$ were specific only to $\mathrm{B}$-samples showing outstanding performances. The increased amount of this species can be attributed to the enlarged exposure of $\{001\}$ facets which, in turn, opens the way for a more efficient charge separation process induced by the synergism between these and $\{101\}$-oriented crystallographic surfaces. In accordance with other research works, $\{001\} /\{101\}$ surface area ratio - detected through the $\mathrm{O}_{\mathrm{T}} \mathrm{H}$ content exhibited an optimum value with respect to photocatalytic activity. By contrast, the quantity of bridging $\mathrm{OH}$-groups $\left(\mathrm{O}_{\mathrm{Br}} \mathrm{H}\right)$ has seemingly no definite interrelation with initial reaction rates. However, considering its crucial roles in charge carrier dynamics and interfacial charge transfer, its presence must be essential for achieving acceptable catalytic efficiency. 
Besides the above, chemical nature of $\mathrm{TiO}_{2}$ surfaces plays fundamental role also elseways in aqueous photocatalytic reactions. It determines the surface charge density of particles which, in turn, has impact on adsorption processes and on the colloidal stability of dispersions. By adjusting the $\mathrm{pH}$ of aqueous suspensions, net particle charges are modified either by protonation or deprotonation of surface hydroxyl $\left(\mathrm{O}_{\mathrm{T}} \mathrm{H}\right.$ - and $\left.\mathrm{O}_{\mathrm{Br}} \mathrm{H}-\right)$ groups. There is a certain $\mathrm{pH}$ at which net charge of most grains reaches zero ( $\mathrm{pH}$ IEP: the $\mathrm{pH}$ of isoelectric point) and, consequently, their mutual coulombic repulsion disappears leading to the system's coagulation. To explore hydrodynamic characteristics of our titania samples, dynamic light scattering (DLS) measurements were performed. As anticipated, $\mathrm{pH}_{\text {IEP }}$ values did not show any strong interdependence with any of the structural parameters (i.e., phase ratio, particle size and morphology), because these properties collectively define acid strengths of both types of OH-groups. Surprisingly, though, no correlation was found between $\mathrm{pH}$ IEP-S and the quality/quantity of surface $\mathrm{OH}$-groups either. This contradiction could not be resolved in lack of suitable theoretical background information. Nevertheless, for our five B-samples investigated, significantly lower $\mathrm{pH}_{\text {IEP }}$ values (2.2-4.0) were obtained than that of P25-B (5.8). Such low $\mathrm{pH}$ IEP-S may greatly contribute to superior catalytic activities under the circumstances of the current aqueous phenol decomposition experiments. Based on measurement and calculation results, the $\mathrm{pH}$ of suspensions stayed in the range of $c a$. 6.6 to 3.5 throughout the entire reaction, thus assuring relatively high colloidal stability and effective catalyst surface. Hydrodynamic agglomerate diameters ( $\left.\mathrm{d}_{\mathrm{DLS}}\right)$ recorded at around $\mathrm{pH} 6$ have provided somewhat consistent data: $\mathrm{d}_{\mathrm{DLS}}$ of most of our samples fell within 540 and $620 \mathrm{~nm}$, whereas P25-B had a remarkably higher value of $900 \mathrm{~nm}$. B-1.41-355-30 was the only exception amongst B-samples, for which, despite its lowest $\mathrm{pH}$ IEP, a $\mathrm{d}_{\mathrm{DLS}}$ as high as 820 $\mathrm{nm}$ was measured. These apparently conflicting values might arise from the unique chemical quality of this catalyst, as appreciable amount of oxygen vacancy is incorporated in its crystal lattice. Subsequent test tube sedimentation experiments have provided similar results to the above findings. Here, most B-sample dispersions $\left(\mathrm{c}_{\mathrm{TiO} 2}=1 \mathrm{~g} / \mathrm{L}\right)$ displayed outstanding stability even after 72 hours of storage at their natural pH-s (ca. 4.8 to 6.6), while B-1.41-355-30 discernibly started coagulating already after 3 hours. Despite its highest $\mathrm{d}_{\mathrm{DLS}}$ and $\mathrm{pH}$ IEP, the turbidity of P25-B's suspension has not dropped so much behind those of the most efficient B-samples.

Dissolved oxygen concentration monitoring was carried out in almost the same reaction system what was applied at the original phenol degradation experiments. Circumstances differed in only two aspects here. On the one hand, the $\mathrm{TiO}_{2}$ content of suspensions had to be reduced to its one fifth $\left(\mathrm{c}_{\mathrm{TiO} 2}\right.$ $=0.2 \mathrm{~g} / \mathrm{L}$ ) to set a reliably measurable oxygen depletion rate. Secondly, the reactor was completely sealed off after saturating the suspension to the appropriate oxygen level (8.25 ppm). Since oxygen acts as the primary electron acceptor in these chemical transformations, its consumption rate $\left(\mathrm{r}_{0,02}\right)$ must be proportional to the photocatalytic efficiency. Beyond this advantageous feature, this 
technique is fast and cheap, therefore we recommend its application for such routine comparative investigations, where mechanisms of the given model reaction have already been explored. As suspected in advance, no strong correlation was found between $\mathrm{r}_{0, \mathrm{O} 2}$ and the initial decomposition rate of phenol ( $\mathrm{r}_{0}$,phenol $)$ since $\mathrm{O}_{2}$ is consumed also for the degradation of intermediates. However, if comparing $\mathrm{r}_{0, \text { phenol }}$ to the specific surface area- (SSA) and catalyst loading-normalized value of $\mathrm{r}_{0, \mathrm{O} 2}$ (i.e., $\left.\mathrm{r}_{0, \mathrm{O} 2} /\left(\mathrm{SSA} \times \mathrm{c}_{\mathrm{TiO} 2}\right)\right)$, a quasi-linear interrelation can be seen in between. This is quite plausible since $\mathrm{r}_{0, \mathrm{O} 2} /\left(\mathrm{SSA} \times \mathrm{c}_{\mathrm{TiO} 2}\right)$ basically describes a surface area-independent photocatalytic efficiency, which definitely has a closer connection with the initial breakdown rate of phenol. In the early stages of the decomposition, the concentration of different intermediates may still be relatively low, therefore, the reaction predominantly advances via phenol-radical interactions.

Total organic carbon (TOC) measurements were performed on three catalysts - including B1.00-355-30, B-1.24-355-30/2 and P25-B - in order to compare their mineralization efficiencies. Here, the same aliquots were investigated that had been sampled for HPLC studies. Latter results perfectly complemented the information gathered from TOC as they provided us with concentration profiles of phenol and of aromatic intermediates (pyrocatechol: PC; hydroquinone: HQ). The abovementioned B-samples were consciously selected for TOC experiments; B-1.24-355-30/2 showed the highest phenol degradation rate, while B-1.00-355-30 has almost identical material characteristics (SSA, particle size, phase composition), $\mathrm{r}_{0, \text { phenol }}$ and $\mathrm{r}_{0,02}$ like $\mathrm{P} 25-\mathrm{B}$ has. According to the expectations, B-1.00-355-30 and P25-B displayed very similar TOC decays, whereas B-1.24-355$30 / 2$ behaved differently. It can clearly be deduced from the findings that its mineralization rate is basically restrained by its low SSA which is less than half the SSA of the other two catalysts. Nevertheless, despite this limitation, the TOC drop rate of B-1.24-355-30/2 does not fall so much behind. Moreover, it turned out from phenol degradation profiles that its higher radical production efficiency is preserved longer compared to B-1.00-355-30 and P25-B, though an opposite tendency could have rather been expected due to the presumably higher surface concentration of intermediates. 


\section{6. Összefoglalás}

Munkánk során kifejlesztettünk két $\mathrm{H}_{2}$-levegő gázeleggyel táplált diffúziós lángreaktort (A- és B-égők) és ezek perifériális elemeit (kvarckémény, $\mathrm{TiO}_{2}$-gyüjtő, prekurzortartály), amelyek együttesen alkalmasnak bizonyultak nanoméretü $\mathrm{TiO}_{2}$ katalizátorok elöállítására. Ezen eszközök stabil és jól kontrollálható szintéziskörülményeket biztosítottak, ami megmutatkozott a termékek anyagjellemzőinek és fotokatalitikus aktivitásainak megfelelő reprodukálhatóságában.

Az A-égő konstrukciós hibáit a B-égö elkészítésekor kiküszöböltük. A reaktorok kissé eltérő felépítésének köszönhetően lehetőségünk nyílt összehasonlítani az egyes szintézisparamétereknek a katalizátorok elsődleges szerkezeti sajátságaira gyakorolt hatását, valamint ezek tendenciáit. Az ebből származó adatok jelentősen hozzájárultak azon alapvető részecskedinamikai folyamatok megértéséhez, amelyek végső soron meghatározzák a keletkező $\mathrm{TiO}_{2}$ minták anyagtulajdonságait. $\mathrm{Az}$ ezzel kapcsolatos - az irodalmi következtetésekkel összhangban álló - konklúziókat az alábbi pontokban összegezzük aszerint rendszerezve, hogy melyik reaktornál tett megfigyelésről esik szó. A/B-égö: A kísérleti adatok közvetett bizonyítékot szolgáltattak arra vonatkozóan, hogy rutil részecskék kizárólag a korábban létrejött anatáz klaszterekből alakulhatnak ki. A rutil krisztallitok ugyanis mindig nagyobb mérettartományba estek, mint az azonos minta anatáz részecskéi. Az egyetlen kivételes eset körülményeit a következő pontban ismertetjük.

$B$-égő: Kimutattuk, hogy a $\mathrm{H}_{2}-\mathrm{O}_{2}$ mólarány növelése a lánghőmérséklet emelkedéséből adódóan egyre nagyobb részecskeméretet, diszperzitásfokot és rutiltartalmat eredményez. A rutilméret csak két, az általunk használt paramétertartomány felső szegmenséből származó minta esetén mutatott csökkenő tendenciát. Ennek oka, hogy a létrejött oxigénszegény környezet hatására a kristályrácsban oxigén-hibahelyek jelennek meg, melyek elősegítik az anatáz $\rightarrow$ rutil fázisátalakulást. Következésképpen a rutil részecskék egyre kisebb méretű klaszterekből kezdenek el kifejlődni, mint kevésbé reduktív körülmények között.

B-égő: Az A-égőnél tapasztaltakhoz hasonlóan a prekurzorbeviteli sebesség növelésének kezdeti szakaszában az anatáz részecskék egyre nagyobbak lesznek, míg az anatáztartalom gyakorlatilag változatlan marad. $\mathrm{Ez}$ a viselkedés a $\mathrm{TiCl}_{4}$ relatíve konstans gázfázisú koncentrációjával magyarázható, ami viszont együtt jár a lánghőmérséklet fokozatos növekedésével. Az egységnyi térfogatelemben egységnyi idő alatt átalakuló molekulák száma ugyanis egyre nagyobb lesz a térfogatáram emelésével. Egy küszöbértéken túl - amelyet az A-égő esetén nem sikerült meghaladni - a prekurzor-levegő elegy azonban elkezd elszegényedni, ettől a ponttól kezdve pedig a minták anyagtulajdonság-változásai is a várakozásoknak megfelelően alakulnak. Nevezetesen a krisztallitméretek csökkenése mellett az anatáztartalom is növekedésnek indul, mivel ebben a betáplálási tartományban már a részecskék tartózkodási ideje válik meghatározóvá. Ezt a hatást 
tovább erősíti a lánghőmérséklet csökkenése, melynek hátterében a prekurzorkoncentráció csökkenése, valamint az egyre nagyobb térfogatáram által kiváltott növekvő hődisszipáció áll.

A/B-égő: A reakciótéren keresztülhaladó gázáramok $\left(\mathrm{H}_{2}\right.$, levegö, $\mathrm{TiCl}_{4}$-betáplálás) kölcsönös zavaró hatása miatt a keletkező anyagok sajátságai esetenként szignifikáns eltérést mutattak egy ideális szintézisrendszerből (ahol minden változó tökéletesen független egymástól azok teljes müködési tartományában) származó minták elméleti alapon várható tulajdonságaihoz képest. Ebben olyan tényezők szerepét sikerült azonosítanunk, mint például az égők geometriája valamint az egyes gázáramok értéke és azok aránya.

A-égő: $\mathrm{A} \mathrm{TiCl}_{4}$ kémiai átalakulása során felszabaduló hőenergia bizonyos esetekben összemérhető a lángot létrehozó reakciók teljes entalpiájával, így az nagymértékben befolyásolhatja a reakciótér hőmérsékletének térbeli eloszlását. Következésképpen a gázfázisbeli prekurzorkoncentráció alkalmas megválasztásával jelentősen javítható a szintézisfolyamat kontrollálhatósága.

$B$-égő: Tanulmányoztuk a gázfázisbeli prekurzorkoncentráció növelésének független hatását is. Ennek érdekében megnöveltük a $\mathrm{TiCl}_{4}$-tartály hőmérsékletét - két külön szintézisben, először 50 majd $70^{\circ} \mathrm{C}-\mathrm{ra}$-, miközben minden egyéb paramétert (az egyes gázáramokat) a legnagyobb aktivitással rendelkező mintánk (B-1.24-355-30/2) értékeire állítottunk. Ez a megvalósítás a prekurzor intenzívebb párolgását idézi elő, melynek eredményeképpen a lánghőmérséklet és a reakciótérbe kerülő molekulák/klaszterek ütközési gyakoriságának növekedése lenne várható. Érdekes módon azonban, míg az elsődleges anyagtulajdonságok változása (részecskeméret, fázisösszetétel) nagyjából a mérési hibahatáron belül maradt, a fotokatalitikus aktivitás 25\%-os csökkenést mutatott (B-1.24-355-70) a kiindulási állapothoz (B-1.24-355-30/2) képest. A jelenség hátterében meghúzódó okok felderítése céljából a későbbiekben tárgyalt anyag- és felületvizsgálati módszereket alkalmaztunk.

$B$-égő: A rendelkezésre álló paraméterváltoztatási opciók közül utolsóként az égőn áthaladó teljes gázáram módosítását vizsgáltuk. Ennek során két egymást követő kísérletben állítottunk elő mintákat oly módon, hogy az egyik esetben egyidejűleg 20\%-kal növeltük, a másikban pedig 20\%-kal csökkentettük a hidrogén és a levegő áramlási sebességét a leghatékonyabb minta (B-1.24-355-30/2) paramétereihez képest. Mindeközben a $\mathrm{TiCl}_{4}$-betáplálás mértékét nem módosítottuk, így a prekurzor tömegárama valamint a $\mathrm{H}_{2}-\mathrm{O}_{2}$ mólarány minden mintánál azonos volt. Számításaink alapján valószínűsíthető továbbá, hogy a reakciótérben kialakuló áramlási viszonyok sem változtak számottevően. Ilyen körülmények között egyedül a lánghőmérséklet változása várható amint csökkent illetve nőtt az időegység alatt elreagált tüzelőanyag mennyisége. A szintéziseket követő vizsgálatok kimutatták, hogy a teljes gázáram emelésével a rutilméret valóban növekszik, viszont az anatáz részecskéknél nem figyelhető meg ilyen tendencia. A fotokatalitikus aktivitás mindkét esetben körülbelül 19\%-kal csökkent a kiindulási értékhez viszonyítva. 
A lángszintézis-módszer kifejlesztése mellett munkánk másik fontos célja volt annak megértése, hogy döntően mely anyagtulajdonságok határozzák meg a $\mathrm{TiO}_{2}$ minták fotokatalitikus aktivitását. Ezen ismeretek ugyanis a teljes szintézisparaméterek-mintajellemzők összefüggésrendszer feltérképezésével lehetőséget nyújthatnak a fotokatalizátorok hatékonyságnövelését célzó tudatos szintézistervezésnek. Ennek megfelelően korrelációanalízist végeztünk az A-égő paraméteroptimalizálási kísérleteiből $\left(\mathrm{H}_{2}-\mathrm{O}_{2}\right.$ mólarány, TiCl $\mathrm{T}_{4}$-betáplálási sebesség) származó két mintasorozat összes elemének aktivitás-anyagtulajdonság adathalmazán. Az eredmények alapján arra következtettünk, hogy önmagában sem a részecskeméret sem a fázisösszetétel nem tehető felelőssé az egyes minták aktivitásai között tapasztalt nagymértékü eltérésért. A legszemléletesebb példát erre vonatkozóan az A-1.21-237-30 és az A-0.91-237-30 jelzésű minták összehasonlítása szolgáltatja. Annak ellenére, hogy anatáztartalmuk gyakorlatilag megegyezik és ugyanezen részecskéik átlagos átmérői is csak mintegy 5 nm-nyi különbséget mutatnak, utóbbi aktivitása 2.4-szerese az előbbiének. Hasonló nagyságrendű eltérések találhatóak a prekurzorbeviteli sebesség változtatásával előállított bizonyos mintapárok esetén is. A későbbi TEMvizsgálatok eredményei adtak végül magyarázatot a fenti megfigyelésekkel kapcsolatosan. Kiderült ugyanis, hogy a leginkább poliéderes nanorészecskéket tartalmazó minták nagyobb aktivitással rendelkeznek, mint a jellemzően gömb alakú krisztallitokból álló katalizátorok. A részecskék alakfüggő aktivitása jól ismert jelenség heterogén katalízis területén, ami a különböző kristálylapok eltérő felületi arányának tulajdonítható.

Fontos kérdés, hogy mely szintézisparaméterek és milyen módon befolyásolják a részecskemorfológiát. Erre a prekurzorbeviteli sebesség és a $\mathrm{H}_{2}-\mathrm{O}_{2}$ mólarány változtatásával előállított B-minták elemzése adja meg a választ. Az adatok alapján jól látszik, hogy a poliéderes részecskék kialakulásának az alacsonyabb lánghőmérséklet és/vagy a kisebb tartózkodási idő kedvez. Ezek a tendenciák A-égő mintasorozatainál nem figyelhetők meg, részben a helytelen kísérlettervezés $\left(\mathrm{H}_{2}-\mathrm{O}_{2}\right.$ mólarány szabályzás), részben pedig a gázfázisbeli $\mathrm{TiCl}_{4}$-koncentráció nem várt ingadozásának köszönhetően.

Vizsgálataink egy korai szakaszában öt darab, a prekurzoráram szabályzásával készült A-mintát és a P25-B jelü referenciakatalizátort gázfázisú fotokatalitikus bontási teszteknek is alávetettük annak érdekében, hogy mélyebb bepillantást nyerhessünk mintáink müködési mechanizmusát illetően és közelebb kerüljünk változatos aktivitásuk okainak megértéséhez. Ebből a célból két gázhalmazállapotú modellvegyület - metanol és acetaldehid - bomlási viselkedését tanulmányoztuk. A vizes közegü és gázfázisú reakciók lényegesen különböző fizikai-kémiai körülményei ellenére az egyes minták metanolbontási sebessége csaknem ugyanazt a sorrendet követi, mint a fenol esetében. Ez azt is jelenti, hogy az aktivitások rendre nagyobbak azoknál a mintáknál, amelyek több poliéderes részecskét tartalmaznak. Ezzel szemben az acetaldehid esetén egy kevésbé egyértelmü, de ellentétes 
tendencia rajzolódott ki. Ésszerü lehet a feltételezés, miszerint a fent említett hasonlóság a modellvegyületek hasonló bomlási mechanizmusának a következménye. A fenol hidrofób jellegéböl adódóan kevésbé adszorbeálódik $\mathrm{TiO}_{2}$-on, így lebontásában döntő szerephez jutnak a molekulákat elérő gyökök. Habár gázfázisban a metanol alapvetően erősen kötődik a $\mathrm{TiO}_{2}$ felületéhez, a reakciórendszerben megtalálható nagy mennyiségü víz és a keletkező köztitermékek képesek visszaszorítani az adszorpcióját. Emiatt, a fenollal analóg módon, a metanol degradációja is jellemzően gyökökkel történő kölcsönhatás útján megy végbe. Mindezen összefüggések arra engednek következtetni, hogy a poliéderes részecskék gyökgenerálási hatékonysága számottevően nagyobb, mint a gömbalakúaké. Az acetaldehid bomlásánál - ahol csak gyenge korreláció volt megfigyelhető a poliéderes részecsketartalommal - elsősorban a közvetlen lyuk-oxidáció dominál. Itt, amint az a bomlásgörbék lefutásából is kitünik, a nagyobb fajlagos felület elönyösebbnek bizonyult a nagyobb aktivitás megőrzésében.

Míg az A-égő mintáinak aktivitásai bizonyos mértékben korreláltak a részecskemorfológiával, a B-minták szerkezeti sajátságainak (alak, méret, fázisarány) egyike sem mutatott egyértelmü összefüggést a katalizátorok hatékonyságával. Ezen jellemzők ugyanis együttesen, azonban a különböző esetekben változó mértékü hozzájárulással határozzák meg a fotokatalitikus aktivitást. Hatásuk pedig gyakran indirekt módon nyilvánul meg olyan tényezőkön keresztül, mint például a $\mathrm{TiO}_{2}$ felületének kémiai minősége. Ennek tanulmányozásához röntgen fotoelektron-spektroszkópiai (XPS) méréseket végeztünk néhány A- és B-mintán, valamint a P25-B katalizátoron. A Ti 2p és O 1s kötési energiákhoz tartozó csúcsok mennyiségi analízisével információt nyerhettünk a $\mathrm{Ti}^{4+} / \mathrm{Ti}^{3+}$ és a hidroxil/oxid (vagy rács) típusú oxigénatomok felületi arányát illetően. Az eredmények azt mutatták, hogy a $\mathrm{Ti}^{3+}$ oxidációs állapot egyik vizsgált mintában sem volt megtalálható. Ezenkívül a hidroxilcsoportok jelkettőse mellett két további, ismeretlen $\mathrm{O}$ 1s csúcsot detektáltunk a rácsoxigén alacsony kötési energiájú oldalán. Ezek eredetével kapcsolatban semmiféle információ nem szerepel a szakirodalomban, ezért nem képezték részét a későbbi kiértékeléseknek. Habár a kétféle felületi OH-csoport (terminális és híd: ”bridging”) összesített oxigénatom-aránya nem korrelált egyik anyagtulajdonsággal sem, a terminális hidroxilcsoportok $\left(\mathrm{O}_{\mathrm{T}} \mathrm{H}\right)$ kizárólag a vizsgált mintabázison belül kiemelkedő aktivitással bíró B-minták esetén voltak jelen. Az $\mathrm{O}_{\mathrm{T}} \mathrm{H}$-csoportok nagyobb sürüsége a $\{001\}$ orientációjú kristálylapok nagyobb felületi arányával magyarázható, amelyek az \{101\}-es lapokkal kialakuló szinergikus kölcsönhatás révén hatékonyabb töltésszeparációt tesznek lehetővé. Összhangban más kutatási eredményekkel, az $\mathrm{O}_{\mathrm{T}} \mathrm{H}$-csoportok mennyiségéböl származtatható $\{001\} /\{101\}$ felületarány az aktivitás tekintetében optimummal rendelkezik. A híd OH-csoportok $\left(\mathrm{O}_{\mathrm{Br}} \mathrm{H}\right)$ sürüsége és a kezdeti reakciósebességek között viszont látszólag nincs határozott kapcsolat. Mindazonáltal figyelembe véve az $\mathrm{O}_{\mathrm{Br}} \mathrm{H}$-csoportok töltéshordozó-dinamikában és határfelületi 
töltéstranszfer-folyamatokban betöltött kulcsszerepét, jelenlétük kétségkívül létfontosságú a megfelelő fotokatalitikus hatásfok eléréséhez.

A $\mathrm{TiO}_{2}$ felületek kémiai minősége a fentiek mellet egyéb módokon is meghatározó jelentőséggel bír a vizes közegü fotokatalitikus reakciókban. A részecskék felületi töltéssürüségén keresztül ugyanis befolyásolja az adszorpciós folyamatokat és a kolloidstabilitást. A szuszpenziók kémhatásának változtatásával módosul a hidroxilcsoportok $\left(\mathrm{O}_{\mathrm{T}} \mathrm{H}\right.$ és $\left.\mathrm{O}_{\mathrm{Br}} \mathrm{H}\right)$ protonáltsági állapota, ezáltal pedig a részecskék teljes felületi töltése. Utóbbi egy bizonyos $\mathrm{pH}-\mathrm{n}$ nullává válik $\left(\mathrm{pH}_{\mathrm{IEP}}\right.$ : izoelektromos pont), ami a krisztallitok között addig meglévő Coulomb-taszítás megszünéséhez és így a rendszer koagulációjához vezet. Mintáink hidrodinamikai jellemzőinek feltérképezését dinamikus fényszórásméréssel (DLS) végeztük. Az előzetes várakozásoknak megfelelően a $\mathrm{pH}_{\mathrm{IEP}}$ értékek nem mutattak összefüggést a szerkezeti paraméterekkel, hiszen ezen sajátságok együttesen határozzák meg mindkét típusú hidroxilcsoport savasságát. Érdekes módon a $\mathrm{pH}_{\mathrm{IEP}-\mathrm{k}} \mathrm{a}$ hidroxilcsoportok mennyiségével/minőségével sem korreláltak, ezt az ellentmondást azonban megfelelő elméleti háttérinformáció hiányában nem sikerült feloldani. A vizsgáltban részt vevő öt darab B-minta lényegesen kisebb $\mathrm{pH}_{\mathrm{IEP}}$ értékeket produkált (2.2-4.0), mint a P25-B referenciakatalizátor (5.8). Ez az alkalmazott vizes közegü fenolbontás körülményeit figyelembe véve jelentős mértékben hozzájárulhatott mintáink nagyobb aktivitásához. Mérési és számítási eredményeink alapján ugyanis a szuszpenziók pH-ja körülbelül 6.6 és 3.5 között maradt a reakciók teljes időtartamában, ami relatíve nagy kolloidstabilitást és ezáltal nagy effektív katalizátorfelületet biztosított. Az agglomerátumok 6-os $\mathrm{pH}$ közelében felvett hidrodinamikai átmérői ( $\mathrm{d}_{\mathrm{DLS}}$ ) jól illeszkedtek az előző adatokhoz: mintáink nagy részének dDLS értékei 540 és 620 nm közé estek, míg a P25-B esetén ez 900 nm-nek adódott. Az egyetlen kivétel a B-1.41-355-30 jelü minta volt, amely legkisebb $\mathrm{pH}_{\mathrm{IEP}}$ értéke ellenére $820 \mathrm{~nm}$-es aggregátumokat tartalmazott. Ezek az ellentmondó eredmények valószínleg az egyedülálló kémiai minőségnek köszönhetők, mivel ez a minta nagy mennyiségű oxigén-hibahelyet tartalmazott. Az eddig bemutatott mérési adatok helyességét sikerült igazolnunk szedimentációs kísérletekkel is. A B-mintákból készült szuszpenziók $\left(\mathrm{c}_{\mathrm{TiO} 2}=1 \mathrm{~g} / \mathrm{l}\right)$ többsége még 72 óra elteltével is kiemelkedő stabilitással bírt természetes pH-n (4.8-6.6), ezzel szemben a B-1.41-355-30 jelü minta már 3 óra után elkezdett koagulálódni. A P25-B szuszpenziójának turbiditása magas $\mathrm{pH}_{\mathrm{IEP}}$ és $\mathrm{d}_{\mathrm{DLS}}$ értékei ellenére nem volt számottevően alacsonyabb, mint a leghatékonyabb B-mintáké.

Az oldott oxigénkoncentráció meghatározása a fenolbontásnál alkalmazott reakciórendszerrel szinte azonos körülmények között történt, két tényező kivételével. Egyrészt a szuszpenziók szokásos $\mathrm{TiO}_{2}$ tartalmát az ötödére kellett csökkenteni $\left(\mathrm{c}_{\mathrm{TiO} 2}=0.2 \mathrm{~g} / \mathrm{l}\right)$ annak érdekében, hogy megbízhatóan mérhető oxigénfogyás-sebességet kapjunk. Továbbá nyilvánvalóan szükségessé vált a reaktor tökéletes lezárása is, amelyre a szuszpenziók megfelelő oldott oxigénszintre (8.25 ppm) történő 
szaturációját követően került sor. Mivel a vizsgált kémiai folyamatokban az oxigén az elsődleges elektronakceptor szerepét tölti be, koncentrációcsökkenésének mértéke $\left(\mathrm{r}_{0,02}\right)$ arányos a fotokatalitikus hatékonysággal. Ezen előnyös tulajdonsága mellett a módszer ráadásul olcsó és gyors, ezért használata javasolt olyan rutinjellegü összehasonlító vizsgálatokhoz, ahol az adott modellreakció mechanizmusa már teljes egészében ismert. A rendszerben jelenlévő oxigén mennyiségét a köztitermékek bomlása is csökkenti, így elözetesen már sejthető volt, hogy nem találunk korrelációt a fenolbomlás kezdeti sebessége $\left(\mathrm{r}_{0, \text { fenol }}\right)$ és az $\mathrm{r}_{0, \mathrm{O} 2}$ között. Azonban ha az $\mathrm{r}_{0, \mathrm{O} 2}$ fajlagos felülettel $(\mathrm{SSA})$ és katalizátortartalommal normált alakját (vagyis: $\left(\mathrm{r}_{0, \mathrm{O} 2} /\left(\mathrm{SSA} \times \mathrm{c}_{\mathrm{TiO} 2}\right)\right)$

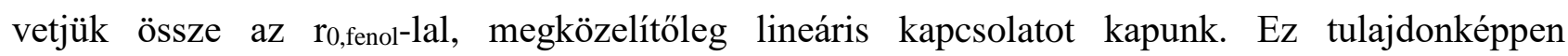
kézenfekvőnek tekinthető, mert az $\mathrm{r}_{0, \mathrm{O} 2} /\left(\mathrm{SSA} \times \mathrm{c}_{\mathrm{TiO} 2}\right)$ által reprezentált fajlagos felület-független fotokatalitikus hatékonyság összefüggést kell mutasson a fenol kezdeti bomlássebességével. A bontási folyamat korai szakaszában ugyanis a különböző köztitermékek koncentrációja jó eséllyel kellően alacsony ahhoz, hogy túlnyomórészt még a fenol-gyök kölcsönhatások domináljanak.

Három különböző katalizátor - B-1.00-355-30, B-1.24-355-30/2 és a P25-B - mineralizációs hatékonyságának összehasonlítása céljából teljes szerves széntartalom (TOC) méréseket végeztünk. A vizsgált oldatminták részleteit ezzel párhuzamosan HPLC analízisnek is alávetettünk. Utóbbi módszer segítségével származtattuk a fenol és elsődleges aromás köztitermékeinek (pirokatechin: PC; hidrokinon: HQ) koncentrációprofiljait, amelyek jól kiegészítették a TOC-ból kinyerhető információkat. A fenti B-minták kiválasztása tudatosan történt; a B-1.24-355-30/2 jelü produkálta a legnagyobb fenolbontási sebességet, míg a B-1.00-355-30 minta szinte teljesen ugyanolyan anyagtulajdonságokkal (SSA, részecskeméret, fázisarány), valamint $r_{0}$,fenol és $r_{0,02}$ értékekkel rendelkezett, mint a P25-B. A várakozásoknak megfelelően a B-1.00-355-30 és a P25-B katalizátorok nagyon hasonló TOC-csökkenést mutattak. Az adatokból látszik, hogy a B-1.24-355-30/2 mineralizációs teljesítményét kis fajlagos felülete korlátozza, ami a másik két minta SSA értékének nem egészen a fele volt. Mindazonáltal a B-1.24-355-30/2 minta TOC-csökkenésének sebessége nem volt jelentősen kisebb a többinél. Ráadásul amint az a fenolkoncentráció-profilokból kiderült, nagyobb gyökgenerálási hatékonysága hosszabb ideig megőrződött, holott a köztitermékek vélhetően magasabb felületi koncentrációja miatt inkább annak csökkenése lett volna várható. 


\section{References}

[1] O. Carp, C.L. Huisman, A. Reller, Prog. Solid State Chem. 32 (2004) 33-177.

[2] K. Wetchakun, N. Wetchakun, S. Sakulsermsuk, J. Ind. Eng. Chem. 71 (2019) 19-49.

[3] F. Piccinno, F. Gottschalk, S. Seeger, B. Nowack, J. Nanopart. Res. 14 (2012) 1109.

[4] Q. Liu, J. Wang, Sol. Energy 184 (2019) 454-465.

[5] M. Fehse, E. Ventosa, Chem. Plus Chem. 80 (2015) 785-795.

[6] C. Wang, L. Yin, L. Zhang, Y. Qi, N. Lun, N. Liu, Langmuir 26 (2010) 12841-12848.

[7] H. Liu, W. Yang, M. Wang, L. Xiao, S. Liu, Sens. Actuators B 29 (2016) 490-498.

[8] L. Caballero, K.A. Whitehead, N.S. Allen, J. Verran, J. Photochem. Photobiol., A 202 (2009) 92-98.

[9] C. McCullagh, J. Robertson, D.W. Bahnemann, P. Robertson, Res. Chem. Intermed. 33 (2007) 359-375.

[10] B. Jalvo, M. Faraldos, A. Bahamonde, R. Rosal, J. Hazard. Mater. 340 (2017) 160-170.

[11] A. Mills, J.S. Wang, J. Photochem. Photobiol., A 182 (2006) 181-186.

[12] M. Smits, C. Chan, T. Tytgat, B. Craeye, N. Costarramone, S. Lacombe, S. Lenaerts, Chem.

Eng. J. 222 (2013) 411-418.

[13] R.K. Nath, M.F.M. Zain, M. Jamil, Renewable Sustainable Energy Rev. 62 (2016) 1184-1194.

[14] H.-J. Kim, Y.-S. Yoon, K.-H. Yang, S.-J. Kwon, Constr. Build. Mater. 199 (2019) 106-114.

[15] E. Jimenez-Relinque, M. Castellote, Constr. Build. Mater. 214 (2019) 1-8.

[16] A. Aghighi, F. Haghighat, Build. Environ. 85 (2015) 114-122.

[17] H. Destaillats, M. Sleiman, D.P. Sullivan, C. Jacquiod, J. Sablayrolles, L. Molins, Appl. Catal., B 128 (2012) 159-170.

[18] G. Wang, S. Chen, H. Yu, X. Quan, J. Hazard. Mater. 299 (2015) 27-34.

[19] Q. Zhang, H. Wang, X. Fan, F. Lv, S. Chen, X. Quan, Surf. Coat. Tech. 298 (2016) 45-52.

[20] C. Byrne, G. Subramanian, S.C. Pillai, J. Environ. Chem. Eng. 6 (2018) 3531-3555.

[21] A. Hakki, R. Dillert, D.W. Bahnemann, Catal. Today 144 (2009) 154-159.

[22] A. Hakki ,R. Dillert, D.W. Bahnemann, Phys. Chem. Chem. Phys. 15 (2013) 2992-3002.

[23] G. Palmisano, A. Albini, G. Marci, L. Palmisano, D. Ravelli, RSC Energy and Environment

Series No. 15 (2016); Photocatalysis: Applications, Chapter 11

[24] S. Wu, X. Tan, K. Liu, J. Lei, L. Wang, J. Zhang, Catal. Today 355 (2019) 214-220.

[25] S. Liu, J. Yu, M. Jaroniec, J. Am. Chem. Soc. 132 (2010) 11914-11916.

[26] P. Calza, E. Pelizzetti, K. Mogyorósi, R. Kun, I. Dékány, Appl. Catal., B 72 (2007) 314-321.

[27] A.M.T. Silva, C.G. Silva, G. Drazic, J.L. Faria, Catal. Today 144 (2009) 13-18.

[28] T. Boningaria, S.N.R. Inturia, M. Suidanb, P.G. Smirniotisa, J. Mater. Sci. Technol. 34 (2018) 1494-1502.

[29] K. Whitehead, J.I. Hedges, J. Photochem. Photobiol., B 80 (2005) 115-121.

[30] X.M. Zhou, G. Liu, J.G. Yu, W.H. Fan, J. Mater. Chem. 22 (2012) 21337-21354.

[31] A. Takai, P.V. Kamat, ACS Nano 5 (2011) 7369-7376.

[32] B. Wang, H. Xin, X. Li, J. Cheng, G. Yang, F. Nie, Sci. Rep. 4 (2014) 3729-3737.

[33] A. Fujishima, K. Honda, Nature 238 (1972) 37-38.

[34] S.N. Frank, A.J. Bard, J. Am. Chem. Soc. 99 (1977) 303-304.

[35] A.L. Pruden, D.F. Ollis, J. Catal. 82 (1983) 404-417.

[36] T. Matsunaga, R. Tomato, T. Nakajima, H. Wake, FEMS Microbiol. Lett. 29 (1985) 211-214.

[37] D.F. Ollis, H. Al-Ekabi, Eds. Elsevier Science Publishers: Amsterdam 1993 p. 39.

[38] U. Diebold, Surf. Sci. Rep. 48 (2003) 53-229.

[39] A. Fujishima, X. Zhang, D.A. Tryk, Surf. Sci. Rep. 63 (2008) 515-582.

[40] S.M. Sze, Physics of Semiconductor Devices, (1981) Wiley: New York, Chapter 1

[41] S. Kohtani, A. Kawashima, H. Miyabe, Catalysts 7 (2017) 303-318.

[42] J. Schneider, M. Matsuoka, M. Takeuchi, J. Zhang, Y. Horiuchi, M. Anpo, D. W. Bahnemann,

Chem. Rev. 114 (2014) 9919-9986. 
[43] A. Furube, T. Asahi, H. Masuhara, H. Yamashita, M. Anpo J. Phys. Chem. B 103 (1999) 31203127.

[44] T. Yoshihara, R. Katoh, A. Furube, Y. Tamaki, M. Murai, K. Hara, S. Murata, H. Arakawa, M. Tachiya, J. Phys. Chem. B 108 (2004) 3817-3823.

[45] S.T. Martin, H. Herrmann, M.R. Hoffmann, J. Chem. Soc., Faraday Trans. 90 (1994) 33233330.

[46] Y. Tamaki, A. Furube, R. Katoh, M. Murai, K. Hara, H. Arakawa, M. Tachiya, C. R. Chimie 9 (2006) 268-274.

[47] Y. Tamaki, A. Furube, M. Murai, K. Hara, R. Katoh, M. Tachiya, Phys. Chem. Chem. Phys. 9 (2007) 1453-1460.

[48] N. Serpone, D. Lawless, R. Khairutdinov, J. Phys. Chem. 99 (1995) 16646-16654.

[49] N.D. Abazovic, M.I. Comor, M.D. Dramicanin, D.J. Jovanovic, S.P. Ahrenkiel, J.M.

Nedeljkovic, J. Phys. Chem. B 110 (2006) 25366-25370.

[50] S.H. Szczepankiewicz, J.A. Moss, M.R. Hoffmann, J. Phys. Chem. B 106 (2002) 7654-7658.

[51] R.F. Howe, M. Grätzel, J. Phys. Chem. 89 (1985) 4495-4499.

[52] S.H. Szczepankiewicz, A.J. Colussi, M.R. Hoffmann, J. Phys. Chem. B 104 (2000) 9842-9850.

[53] S.H. Szczepankiewicz, J.A. Moss, M.R. Hoffmann, J. Phys. Chem. B 106 (2002) 2922-2927.

[54] M. Grela, A.J. Colussi, J. Phys. Chem. 100 (1996) 18214-18221.

[55] R.F. Howe, M. Grätzel, J. Phys. Chem. 91 (1987) 3906-3909.

[56] O.I. Micic, Y. Zhang, K.R. Cromack, A.D. Trifunac, M.C. Thurnauer, J. Phys. Chem. 97

(1993) 7277-7283.

[57] J.G. Yu, X. J. Zhao, Q. N. Zhao, G. Wang, Mater. Chem. Phys. 68 (2001) 253-259.

[58] D. Lawless, N. Serpone, D. Meisel, J. Phys. Chem. 95 (1991) 5166-5170.

[59] R. Nakamura, T. Okamura, N. Ohashi, A. Imanishi, Y. Nakato, J. Am. Chem. Soc. 127 (2005) 12975-12983.

[60] A. Yamakata, T.-A. Ishibashi, K. Takeshita, H. Onishi, Top. Catal. 35 (2005) 211-216.

[61] M.R. Hoffmann, S.T. Martin, W. Choi, D.W. Bahnemann, Chem. Rev. 95 (1995) 69-96.

[62] B. Ohtani, Catalysts 3 (2013) 942-953.

[63] D.F. Ollis, E. Pelizzetti, N. Serpone, Environ. Sci. Technol. 25 (1991) 1522-1529.

[64] K.H. Wang, H.H. Tsai, Y.H. Hseich, Appl. Catal., B 17 (1998) 313-320.

[65] S. Hager, R. Bauer, G. Kudielka, Chemosphere 41 (2000) 1219-1225.

[66] H. Gerischer Electrochim. Acta 40 (1995) 1277-1281.

[67] K. Tanaka, M.F.V. Capule, T. Hisanaga, Chem. Phys. Lett. 187 (1991) 73-76.

[68] Z. Zhang, C.-C. Wang, R. Zakaria, J.Y. Ying, J. Phys. Chem. B 102 (1998) 10871-10878.

[69] A. Heller, Y. Degani, D.W. Johnson Jr., P.K. Gallagher, J. Phys. Chem. 91 (1987) 5987-5991.

[70] M.A. Henderson, Surf. Sci. Rep. 66 (2011) 185-297.

[71] H. Liu, H.T. Ma, X.Z. Li, W.Z. Li, M. Wu, X.H. Bao, Chemosphere 50 (2003) 39-46.

[72] I. Ilisz, Zs. László, A. Dombi, Appl. Catal., A 180 (1999) 25-33.

[73] I. Ilisz, A. Dombi, Appl. Catal., A 180 (1999) 35-45.

[74] I. Ilisz, K. Főglein, A. Dombi, J. Mol. Catal. A.: Chem. 135 (1998) 55-61.

[75] K. Hashimoto, T. Kawai, T. Sakata, J. Phys. Chem. 88 (1984) 4083-4088.

[76] N. Murakami, Y. Kurihara, T. Tsubota, T. Ohno, J. Phys. Chem. C 113 (2009) 3062-3069.

[77] D.A. Bennett, M. Cargnello, B.T. Diroll, C.B. Murray, J.M. Vohs, Surf. Sci. 654 (2016) 1-7.

[78] W. Chen, Q. Kuang, Q. Wang, Z. Xie, RSC Adv. 5 (2015) 20396-20409.

[79] G. Žerjav, A. Pintar, M. Ferentz, M. Landau, A. Haimovich, A. Goldbourt, M. Herskowitz, Catalysts 9 (2019) 447-465.

[80] W. Choi, A. Termin, M.R. Hoffmann, J. Phys. Chem. 98 (1994) 13669-13679.

[81] K. Wilke, H.D. Breuer, J. Photochem. Photobiol., A 121 (1999) 49-53.

[82] N. Serpone, D. Lawless, J. Disdier, J.M. Hermann, Langmuir 10 (1994) 643-652.

[83] M. Wei, J.M. Hermann, P. Pichat, Catal. Lett. 3 (1989) 73-84.

[84] M.A. Fox, M.T. Dulay, Chem. Rev. 93 (1993) 341-357.

[85] F.B. Li, X.Z. Li, Appl. Catal., A 228 (2002) 15-27. 
[86] D.A. Tryk, A. Fujishima, K. Honda, Electrochim. Acta 45 (2000) 2363-2376.

[87] N. Serpone, E. Pelizzetti, Photocatalysis. Fundamentals and Applications, Wiley \& Sons, New York, 1989

[88] D.F. Ollis, Environ. Sci. Technol. 19 (1985) 480-484.

[89] I. Ilisz, Ph.D. dissertation, 1998, Szeged

[90] H. Al-Ekabi, P. De Mayo, J. Phys. Chem. 89 (1985) 5815-5821.

[91] X. Pang, C. Chen, H. Ji, Y. Che, W. Ma, J. Zhao, Molecules 19 (2014) 16291-16311.

[92] C.S. Turchi, D.F. Ollis, J. Catal 122 (1990) 178-192.

[93] C. Minero, F. Catozzo, E. Pelizzetti, Langmuir 8 (1992) 481-486.

[94] Y. Murakami, E. Kenji, A.Y. Nosaka, Y. Nosaka, J. Phys. Chem. B 110 (2006) 16808-160811.

[95] T. Tatsuma, S.-i. Tachibana, A. Fujishima, J. Phys. Chem. B 105 (2001) 6987-6992.

[96] D.W. Bahnemann, J. Cunningham, M.A. Fox, E. Pelizzetti, P. Pichat, N. Serpone, In: G.R.

Heltz, R.G. Zepp, D.G. Crosby, editors. Aquatic and surface photochemistry. Boca Raton (FL):

Lewis Publishers; 1994, p. 261.

[97] Y. Mao, C. Schoneich, K.D. Asmus, J. Phys. Chem. 95 (1991) 10080-10089.

[98] M.A. Fox, C.C. Chen, Tetrahedron 95 (1991) 5261-5267.

[99] A.V. Emeline, V. Ryabchuk, N. Serpone, J. Photochem. Photobiol., A 133 (2000) 89-97.

[100] D.F. Ollis, C R Acad Sci Paris Serie IIC Chim 3 (2000) 405-411.

[101] M.B. Smith, J. March, Effect of structure on reactivity, advanced organic chemistry: reactions, mechanisms and structure, 5th ed. New York: Wiley; 2001.

[102] J. Araña, E. Tello Rendón, J.M. Doña Rodríguez, J.A. Herrera Melián, O. González Díaz, J. Pérez Peña, Appl. Catal., B 30 (2001) 1-10.

[103] S. Parra, J. Olivero, L. Pacheco, C. Pulgarin, Appl. Catal., B 43 (2003) 293-301.

[104] W.Y. Teoh, L. Mädler, R. Amal, J. Catal. 251 (2007) 271-280.

[105] W.Y. Teoh, F. Denny, R. Amal, D. Friedmann, L. Mädler, S.E. Pratsinis, Top. Catal. 44 (2007) 489-497.

[106] J. Araña, J.M. Doña-Rodríguez, C. Garriga i Cabo, O. González-Díaz, J.A. Herrera-Melián, J. Pérez-Peña, Appl. Catal., B 53 (2004) 221-232.

[107] E. Farfan-Arribas, R.J. Madix Surf. Sci. 544 (2003) 241-260.

[108] L.J. Alemany, M.A. Banares, E. Pardo, F. Martin, M. Galán-Fereres, J.M. Blasco, Appl.

Catal., B. 13 (1996) 289-297.

[109] T.L. Villarreal, R. Gomez, M. Neumann-Spallart, N. Alonso-Vante, P. Salvador, J. Phys. Chem. B 108 (2004) 15172-15181.

[110] S.B. Kim, S.C. Hong, Appl. Catal., B 35 (2002) 305-315.

[111] C.Y. Wang, H. Groenzin, M.J. Schultz, J. Am. Chem. Soc. 126 (2004) 8094-8095.

[112] J. Raskó, J. Kiss, Appl. Catal., A 287 (2005) 252-260.

[113] M. Singh, N. Zhou, D.K. Paul, K.J. Klabunde, J. Catal. 260 (2008) 371-379.

[114] Y. Ohko, D.A. Tryk, K. Hashimoto, A. Fujishima, J. Phys. Chem. 102 (1998) 2699-2704.

[115] M.J. Backes, A.C. Lukaski, D.S. Muggli, Appl. Catal., B 61 (2005) 21-35.

[116] I. Sopyan, M. Watanabe, S. Murasawa, K. Hashimoto, A. Fujishima, J. Photochem.

Photobiol., A 98 (1996) 79-86.

[117] L. Kieu, P. Boyd, H. Idriss, J. Mol. Catal. A: Chem. 176 (2001) 117-125.

[118] Z.F. Pei, V. Ponec, Appl. Surf. Sci. 103 (1996) 171-182.

[119] M.L. Sauer, D.F. Ollis, J. Catal. 158 (1996) 570-582.

[120] E. Obuchi, T. Sakamoto, K. Nakano, Chem. Eng. Sci. 54 (1999) 1525-1530.

[121] H.O. Seo, E.J. Park, I.H. Kim, S.W. Han, B.J. Cha, T.G. Woo, Y.D. Kim, Catal. Today 295 (2017) 102-109.

[122] C.S. Turchi, D.F. Ollis, J. Catal. 119 (1989) 483-496.

[123] J. Kiwi, J. Phys. Chem. 89 (1985) 2437-2439.

[124] M. Anpo, T. Shima, S. Kodama, Y. Kubokawa, J. Phys. Chem. 91 (1987) 4305-4310.

[125] J.M. Hermann, Catal. Today. 53 (1999) 115-129. 
[126] H. Al-Ekabi, A. Safarzadeh-Amiri, W. Sifton, J. Story, Int. J. Environ. Pollut. 1 (1991) 125136.

[127] Y. Luo, D.F. Ollis, J. Catal. 163 (1996) 1-11.

[128] M. Abdullah, G.K.C. Low, R.W. Matthews, J. Phys. Chem. 94 (1990) 6820-6825.

[129] A. Sclafani, L. Palmisano, M. Schiavello, Res. Chem. Intermed. 18 (1992) 211-226.

[130] M. Bideau, B. Claudel, L. Faure, H. Kazouan, J. Photochem. Photobiol., A 61 (1991) 269280.

[131] R.J. Watts, S. Kong, M.P. Orr, G.C. Miller, B.E. Henry, Water. Res. 29 (1995) 95-100.

[132] C. Kormann, D.W. Bahnemann, M.R. Hoffmann, Environ. Sci. Technol. 25 (1991) 494-500.

[133] J.P. Fitts, M.L. Machesky, D.J. Wesolowski, X. Shang, J.D. Kubicki, G.W. Flynn, T.F.

Heinz, K.B. Eisenthal, Chem. Phys. Lett. 411 (2005) 399-403.

[134] P.A. Connor, K.D. Dobson, A.J. McQuillan, Langmuir 15 (1999) 2402-2408.

[135] F. Pellegrino, L. Pellutiè, F. Sordello, C. Minero, E. Ortel, V.-D. Hodoroaba, V. Maurino, Appl. Catal., B 216 (2017) 80-87.

[136] M. Kosmulski, Adv. Coll. Int. Sci. 99 (2002) 255-264.

[137] D. Gumy, S.A. Giraldo, J. Rengifo, C. Pulgarin, Appl. Catal., B 78 (2008) 19-29.

[138] D.W. Bahnemann, S.N. Kholuiskaya, R. Dillert, A.I. Kulak, A.I. Kokorin, Appl. Catal., B 36 (2002) 161-169.

[139] H.R. Eisenhauer, J. Water Pollut. Control Fed. 40 (1968) 1887-1889.

[140] C. Wei, W. Y. Lin, Z. Zainal, N. E. Williams, K. Zhu, A. P. Kruzic, R. L. Smith, K.

Rajeshwar, Environ. Sci. Technol. 28 (1994) 934-938.

[141] J.N. Najm, V.L. Snoeyink, M.T. Suidan, C.H. Lee, Z. Richard, J. Am. Water Works Assoc. 82 (1990) 65-72.

[142] J. Peral, D.F. Ollis, J. Catal. 136 (1992) 554-565.

[143] J. Peral, X. Doménech, D.F. Ollis, J. Chem. Technol. Biotechnol. 70 (1997) 117-140.

[144] A. Mills, S. Le Hunte, J. Photochem. Photobiol., A 108 (1997) 1-35.

[145] E. Piera, J.A. Ayllón, X. Doménech, J. Peral, Catal. Today 76 (2002) 259-270.

[146] J. Peral, D.F. Ollis, J. Mol. Catal. A: Chem. 115 (1997) 347-354.

[147] M.M. Ameen, G.B. Raupp, J. Catal. 184 (1999) 112-122.

[148] L.X. Cao, Z. Gao, S.L. Suib, T.N. Obee, S.O. Hay, J. Freihault, J. Catal. 196 (2000) 253-261.

[149] R.D. Sun, A. Nakajima, T. Watanabe, K. Hashimoto, J. Photochem. Photobiol., A 154 (2003) 203-209.

[150] H. Einaga, S. Futamura, T. Ibusuki, Phys. Chem. Chem. Phys. 1 (1999) 4903-4908.

[151] P.M. Kumar, S. Badrinarajanan, M. Sastry, Thin Solid Films 358 (2000) 122-130.

[152] S. Musić, M. Gotić, M. Ivanda, S. Popović, A. Turković, R. Trojko, A. Sekulić, K. Furić, Mater. Sci. Eng. B 47 (1997) 33-40.

[153] Z. Ambrus, N. Balázs, T. Alapi, Gy. Wittmann, P. Sipos, A. Dombi, K. Mogyorósi, Appl. Catal., B 81 (2008) 27-37.

[154] A.A. Ismail, T.A. Kandiel, D.W. Bahnemann, J. Photochem. Photobiol., A 15 (2010) 183-

193.

[155] H. Zhang, J.F. Banfield, J. Phys. Chem. B 104 (2000) 3481-3487.

[156] R.D. Shannon, J.A. Pask, J. Am. Ceram. Soc. 48 (1965) 391-398.

[157] G. Riegel, J.R. Bolton, J. Phys. Chem. 99 (1995) 4215-4224.

[158] M.J. Jackman, A.G. Thomas, C. Muryn, J. Phys. Chem. C 119 (2015) 13682-13690.

[159] S.S. Watson, D. Beydoun, J.A. Scott, R. Amal, Chem. Eng. J. 95 (2003) 213-220.

[160] D.C. Hurum, A.G. Agrios, K.A. Gray, T. Rajh, M.C. Thurnauer, J. Phys. Chem. B 107 (2003) 4545-4549.

[161] D.C. Hurum, K.A. Gray, T. Rajh, M.C. Thurnauer, J. Phys. Chem. B 109 (2005) 977-980.

[162] B. Ohtani, O.O. Prieto-Mahaney, D. Li, R. Abe, J. Photochem. Photobiol., A 216 (2010) 179-

182.

[163] A. Fujishima, K. Hashimoto, T. Watanabe, TiO2 photocatalysis. Fundaments and applications, 1st ed. Tokyo: BKC; 1999 
[164] S.K. Poznyak, A.I. Kokorin, A.I. Kulak, J. Electroanal. Chem. 442 (1998) 99-105.

[165] M. Iwasaki, M. Hara, S. Ito, J. Mater. Sci. Lett. 17 (1988) 1769-1771.

[166] J. Livage, M. Henry, C. Sanchez, Prog. Solid. State Chem. 18 (1988) 259-341.

[167] C. Guillard, B. Beaugiraud, C. Dutriez, J.M. Herrmann, H. Jaffrezic, N. Jaffrezic-Renault, M. Lacroix, Appl. Catal., B 39 (2002) 331-342.

[168] Y. Takahashi, Y. Matsuoka, J. Mater. Sci. 23 (1988) 2259-2262.

[169] K. Yanagisava, Y. Yamamoto, Q. Feng, N. Yamasaki, J. Mater. Res. 13 (1998) 825-829.

[170] M. Hirano, C. Nakahara, K. Ota, O. Tanaike, M. Inagaki, J. Solid State Chem. 170 (2003) 3947.

[171] D.N. Furlong, C.D. Parfitt, J. Colloid Interface Sci. 65 (1978) 548-554.

[172] V. Pillai, P. Kumar, M.J. Huo, P. Ayyub, D.O. Shah, Adv. Colloid Interface Sci. 55 (1995) 241-269.

[173] I. Zhitomirsky, Mater. Lett. 33 (1998) 305-310.

[174] K. Kamada, M. Mukai, Y. Matsumoto, Electrochim. Acta 47 (2002) 3309-3313.

[175] N. Nagaveni, M.S. Hegde, N. Ravishankar, G.N. Subbanna, G. Madras, Langmuir 20 (2004) 2900-2907.

[176] K.L. Choy, Prog. Mater. Sci. 48 (2003) 57-170.

[177] R. van de Krol, A. Goossens, J. Schoonman, J. Electrochem. Soc. 144 (1997) 1723-1727.

[178] G.L. Messing, S.C. Zhang, G.V. Jayanthi, J. Am. Ceram. Soc. 76 (1993) 2707-2726.

[179] S.E. Pratsinis, Prog. Energy Combust. Sci. 24 (1998) 197-219.

[180] A.P.M. George, E.R. Place, Faraday Symposia of the Chemical Society, 1973, 7, p. 63.

[181] W. Lee, Y. M. Gao, K. Dwight, A. Wold, Mater. Res. Bull. 27 (1992) 685-692.

[182] Y.J. Jang, C. Simer, T. Ohm, Mater. Res. Bull. 41 (2006) 67-77.

[183] H. Schulz, L. Mädler, R. Strobel, R. Jossen, S.E. Pratsinis, T. Johannessen, J. Mater. Res. 20 (2005) 2568-2577.

[184] M.R. Zachariah, S. Huzarewicz, J. Mater. Res. 6 (1991) 264-269.

[185] R. Strobel, A. Baiker, S.E. Pratsinis, Adv. Powder Technol. 17 (2006) 457-480.

[186] S.E. Pratsinis, S. Vemury, Powder Technol. 88 (1996) 267-273.

[187] W.Y. Teoh, L. Mädler, D. Beydoun, S.E. Pratsinis, R. Amal, Chem. Eng. Sci. 60 (2005)

5852-5861.

[188] K.K. Akurati, A. Vital, G. Fortunato, R. Hany, F. Nueesch, T. Graule, Solid State Sci. 9 (2007) 247-257.

[189] K.K. Akurati, A. Vital, U.E. Klotz, B. Bommer, T. Graule, M. Winterer, Powder Technol. 165 (2006) 73-82.

[190] A. Teleki, S.E. Pratsinis, K. Kalyanasundaram, P.I. Gouma, Sensor. Actuat. B-Chem. 119 (2006) 683-690.

[191] J.R. McCormick, B. Zhao, S.A. Rykov, H. Wang, J.G. Chen, J. Phys. Chem. B 108 (2004) 17398-17402.

[192] Y. Zhao, C. Li, X. Liu, F. Gu, J. Alloys Compd. 440 (2007) 281-286.

[193] B. Zhao, K. Uchikawa, J.R. McCormick, C.Y. Ni, J.G. Chen, H. Wang, Proc. Combust. Inst. 30 (2005) 2569-2576.

[194] H.K. Kammler, S.E. Pratsinis, P.W. Morrison Jr., B. Hemmerling, Combust. Flame 128 (2002) 369-381.

[195] G. Skandan, Y.-J. Chen, N. Glumac, B.H. Kear, Nanostruct. Mater. 11 (1999) 149-158.

[196] O.I. Arabi-Katbi, S.E. Pratsinis, P.W. Morrison Jr., C.M. Megaridis, Combust. Flame 124 (2001) 560-572.

[197] C. Almquist, P. Biswas, J. Catal. 212 (2002) 145-156.

[198] P.W. Morrison, Jr., R. Raghavan, A.J. Timpone, C.P. Artelt, S.E. Pratsinis, Chem. Mater. 9 (1997) 2702-2708.

[199] Y. Zhao, C. Li, X. Liu, F. Gu, H. Jiang, W. Shao, L. Zhang, Y. He, Mater. Lett. 61 (2007) 7983.

[200] N.K. Memon. D.H. Anjumb, S.H. Chung, Combust. Flame 160 (2013) 1848-1856. 
[201] O.I. Arabi-Katbi, K. Wegner, S.E. Pratsinis, Ann. Chim. Sci. Mat. 27 (2002) 37-46.

[202] S.E. Pratsinis, W. Zhu, S. Vemury, Powder Technol. 86 (1996) 87-93.

[203] T. Johannessen, S.E. Pratsinis, H. Livbjerg, Powder Technol. 118 (2001) 242-250.

[204] G.D. Ulrich, N.S. Subramanian, Combust. Sci. Technol. 17 (1977) 119-126.

[205] G.D. Ulrich, Combust. Sci. Technol. 4 (1971) 47-57.

[206] T. Matsoukas, S.K. Friedlander, J. Colloid lnterface Sci. 146 (1991) 495-506.

[207] S.K. Friedlander, Smoke, Dust and Haze. Wiley, New York, 1977

[208] S.E. Pratsinis, J. Colloid Interface Sci. 124 (1988) 416-427.

[209] W. Koch, S.K. Friedlander, J. Colloid Interface Sci. 140 (1990) 419-427.

[210] R. Vijayakumar, K.T. Whitby, Aerosol Sci. Technol. 3 (1984) 17-24.

[211] S. Morooka, T. Yasutake, A. Kobata, K. Ikemizu, Y. Kato, Int. Chem. Eng. 29 (1989) 119126.

[212] M. Formenti, F. Juillet, P. Meriaudeau, S.J. Teichner, P. Vergnon, J. Colloid Interface Sci. 39 (1972) 79-89.

[213] G.P. Fotou, S. Vemury, S.E. Pratsinis, Chem. Eng. Sci. 49 (1994) 4939-4948.

[214] W.Y. Teoh, R. Amal, L. Mädler, S.E. Pratsinis, Catal. Today 120 (2007) 203-213.

[215] R. Hong, Z. Ren, J. Ding, H. Li, Chem. Eng. J. (Amsterdam, Heth) 108 (2005) 203-212.

[216] P. Sunsap, D.J. Kim, K.S. Kim, Ind. Eng. Chem. Res. 47 (2008) 2308-2313.

[217] H.D. Jang, S.-K. Kim, Mat. Res. Bull. 36 (2001) 627-637.

[218] J.M. Mäkelä, S. Hellstén, J. Silvonen, M. Vippola, E. Levänen, T. Mäntylä, Mater. Lett. 60 (2006) 530-534.

[219] R.M. Alberici, W.F. Jardim, Appl. Catal., B 14 (1997) 55-68.

[220] B.D. Cullity, Elements of X-ray Diffraction, Addison-Wesley, Reading, 1978.

[221] S. Ma, W. Song, B. Liu, W. Zhong, J. Deng, H. Zheng, J. Liu, X.-Q. Gong, Z. Zhao, Appl. Catal., B 198 (2016) 1-8.

[222] P. Zhang, T. Tachikawa, Z. Bian, T. Majima, Appl. Catal., B 176-177 (2015) 678-686.

[223] N. Tian, Z.-Y. Zhu, S.-G. Sun, Y. Ding, Z.L. Wang, Science 316 (2007) 732-735.

[224] H.B. Jiang, Q. Cuan, C.Z. Wen, J. Xing, D. Wu, X.-Q. Gong, C. Li, H.G. Yang, Angew.

Chem. 50 (2011) 3764-3768.

[225] C.Z. Wen, H.B. Jiang, S.Z. Qiao, H.G. Yang, G.Q.M. Lu, J. Mater. Chem. 21 (2011) 7052-

7061.

[226] A. Selloni, Nat. Mater. 7 (2008) 613-615.

[227] Q. Xiang, J. Yu, M. Jaroniec, Chem. Commun. 47 (2011) 4532-4534.

[228] T. Ohno, K. Sarukawa, M. Matsumura, New J. Chem. 26 (2002) 1167-1170.

[229] H.G. Yang, G. Liu, S.Z. Qiao, C.H. Sun, Y.G. Jin, S. Campbell Smith, J. Zou, H.M. Cheng, G.Q.M. Lu, J. Am. Chem. Soc. 131 (2009) 4078-4083.

[230] Z. He, L. Wen, D. Wang, Y. Xue, Q. Lu, C. Wu, J. Chen, S. Song, Energy Fuels 28 (2014) 3982-3993.

[231] F. Tian, Y. Zhang, J. Zhang, C. Pan, J. Phys. Chem. C 116 (2012) 7515-7519.

[232] http://www.chemspider.com/ (2019.11.10.)

[233] Y. Zhao, C. Li, X. Liu, F. Gu, H.L. Du, L. Shi, Mater. Chem. Phys. 107 (2008) 344-349.

[234] S. Ardizzone, C.L. Bianchi, G. Cappelletti, S. Gialanella, C. Pirola, V. Ragaini, J. Phys.

Chem. C 111 (2007) 13222-13231.

[235] S. Benkoula, O. Sublemontier, M. Patanen, C. Nicolas, F. Sirotti, A. Naitabdi, F. Gaie-Levrel, E. Antonsson, D. Aureau, F.-X. Ouf, S.-I. Wada, A. Etcheberry, K. Ueda, C. Miron, Sci. Rep. 5 (2015) 15088-15098.

[236] P. Krishnan, M. Liu, P.A. Itty, Z. Liu, V. Rheinheimer, M.-H. Zhang, P.J.M. Monteiro, L.E. Yu, Sci. Rep. 7 (2017) 43298-43308.

[237] P.D. Cozzoli, A. Kornowski, H. Weller, J. Am. Chem. Soc. 125 (2003) 14539-14548.

[238] A. Testino, I.R. Bellobono, V. Buscaglia, C. Canevali, M. D’Arienzo, S. Polizzi, R. Scotti, F. Morazzoni, J. Am. Chem. Soc. 129 (2007) 3564-3575.

[239] K. Chhor, J.F. Bocquet, C. Colbeau-Justin, Mater. Chem. Phys. 86 (2004) 123-131. 


\section{Acknowledgements}

First of all, I would like to express my gratitude to Dr. Pál Sipos for giving professional guidance, for always encouraging me and for being a friend during my studies and further. Likewise, I am very grateful to Dr. Károly Mogyorósi, who gave tremendous support in my daily work and from whom I have learnt a lot about the world of tiny particles. I truly thank you both!

I also thank Dr. András Dombi, who provided the chance to work in his research group where I have met very pleasant and professional persons. From this team, I would highlight Dr. Tünde Alapi, Dr. István Ilisz and Katalin Kacsala, their help was essential in my research activity.

I consider myself lucky that I could take part in the life of the Inorganic and Analytical Chemistry Department - led by Dr. Tamás Kiss that time. I always appreciated the friendly and inspiring atmosphere created by the colleagues. I felt like at home in this small community and I brought nice memories from there.

A legnagyobb hálával azonban szüleimnek, testvéremnek, családomnak és Pankámnak tartozom. Szeretetük és odaadó támogatásuk nélkül nem lettem volna képes eljutni idáig. Köszönöm Nektek! 